Florida International University

FIU Digital Commons

$5-20-2019$

\title{
Wavenumber-1 Vortex Rossby Wave Propagation in the Inner Waveguide of a Modeled, Barotropic Nondivergent Tropical Cyclone
}

Israel Gonzalez

igonz008@fiu.edu

Follow this and additional works at: https://digitalcommons.fiu.edu/etd

Part of the Atmospheric Sciences Commons, Earth Sciences Commons, and the Meteorology Commons

\section{Recommended Citation}

Gonzalez, Israel, "Wavenumber-1 Vortex Rossby Wave Propagation in the Inner Waveguide of a Modeled, Barotropic Nondivergent Tropical Cyclone" (2019). FIU Electronic Theses and Dissertations. 4275.

https://digitalcommons.fiu.edu/etd/4275

This work is brought to you for free and open access by the University Graduate School at FIU Digital Commons. It has been accepted for inclusion in FIU Electronic Theses and Dissertations by an authorized administrator of FIU Digital Commons. For more information, please contact dcc@fiu.edu. 


\title{
FLORIDA INTERNATIONAL UNIVERSITY
}

Miami, Florida

\section{WAVENUMBER-1 VORTEX ROSSBY WAVE PROPAGATION IN THE INNER WAVEGUIDE OF A MODELED, BAROTROPIC NONDIVERGENT TROPICAL CYCLONE}

\author{
A dissertation submitted in partial fulfillment of \\ the requirements for the degree of \\ DOCTOR OF PHILOSOPHY \\ in \\ EARTH SYSTEMS SCIENCE \\ by \\ Israel Gonzalez III
}


To: Dean Michael R. Heithaus

College of Arts, Sciences and Education

This dissertation, written by Israel Gonzalez III, and entitled Wavenumber-1 Vortex Rossby Wave Propagation in the Inner Waveguide of a Modeled, Barotropic Nondivergent Tropical Cyclone, having been approved in respect to style and intellectual content, is referred to you for judgment.

We have read this dissertation and recommend that it be approved.

$\begin{array}{r}\text { Robert Burgman } \\ \hline \text { Sundararaman Gopalakrishnan } \\ \hline \text { Seyedmasoud Sadjadi } \\ \hline \text { Hugh E. Willoughby, Major Professor }\end{array}$

Date of Defense: May 20, 2019

The dissertation of Israel Gonzalez III is approved.

Dean Michael R. Heithaus College of Arts, Sciences and Education

Andrés G. Gil Vice President for Research and Economic Development and Dean of the University Graduate School

Florida International University, 2019 
C Copyright 2019 by Israel Gonzalez III All rights reserved. 


\section{ACKNOWLEDGMENTS}

I would first like to acknowledge the National Science Foundation (GS-1724198) for funding this research. Next, I must thank Caridad, Gail, Javier, and Sabrina for their help over the years with any graduate-school-related task that required their attention. My peers also deserve special mention as they have been great to share graduate school experiences with, namely Heather, Javiera, Jeremy, and Maria. My committee members, Gopal, Masoud, Ping, and Rob were a great selection, whom I thank for their honesty, support, criticism, and advice. It is hard to imagine where I would be academically if it were not for meeting my advisor, Hugh Willoughby who has been a fantastic instructor, mentor, collaborator, and friend for over 10 years. Your tutelage has taken me to places I could not have imagined when I chose meteorology. I could not ask for a better advisor. This dissertation is a token of my gratitude.

I want to thank my family for their love, support, and guidance, including my grandparents, and stepmother, Pilar; I love you all. My brother, Johnny deserves first mention because he initiated my joining of the FIU meteorology program when he introduced me to one of his friends (Ponce) and spoke highly of Willoughby and his experiences flying into hurricanes. I wish you great success (Borat voice) with your orthodontic career. Next, I must thank Pilar, a University of Miami alum, for being a great stepmother for nearly TWENTY years. My mother deserves unlimited gratitude for her unwavering love and being an unbelievable provider. Continue to enjoy your muchdeserved retirement and Happy Mother's Day. Lastly, my dissertation completion occurs on the year he turned SIXTY. Consider this a belated birthday gift. Thank you for pushing me and having the confidence in my ability to do great things, you were right. 


\begin{abstract}
OF THE DISSERTATION
WAVENUMBER-1 VORTEX ROSSBY WAVE PROPAGATION IN THE INNER

WAVEGUIDE OF A MODELED, BAROTROPIC NONDIVERGENT TROPICAL

CYCLONE

by
\end{abstract}

Israel Gonzalez III

Florida International University, 2018

Miami, Florida

Professor Hugh E. Willoughby, Major Professor

Vortex Rossby waves (VRWs) have been shown to influence tropical cyclone (TC) structure and intensity change. However, the role of VRWs in TC motion and analyses of the inner waveguide within which the waves propagate have received limited attention. Therefore this dissertation primarily focuses on modeling wavenumber-1 VRWs in a barotropic, nondivergent context to investigate TC-like vortex motion, acquire deeper understanding of propagation within the widest possible inner waveguide, and compare with higher-wavenumber studies.

A mass source-sink pair rotating with a specified frequency is imposed in a mean vortex's eyewall to excite VRWs. Forced waves manifest as vorticity filaments that accumulate at an outer critical radius to produce a ring of trailing spirals that resemble observed TC rainbands. Within the inner waveguide, inward-propagating waves are Doppler-shifted to the cutoff frequency, reflect from a turning point, propagate outward, and are ultimately absorbed at a critical radius. The specified frequency dictates how far VRWs can propagate. Meanwhile, the vortex center exhibits trochoidal motion, 
resembling observed TC eye wobbles. Orbital speed and track depend upon the specified frequency. Lastly, VRWs produce angular momentum and energy fluxes. The former accelerates the mean flow at the radius of maximum wind.

Model sensitivity studies are also undertaken to gain additional insight into VRW dynamics. The first set of experiments adjusts relevant forcing parameters and performs beta-plane simulations to determine the vortex response. The second set adjusts vortex parameters to demonstrate that TC intensity can also influence VRW propagation. Additionally, modeling TC-like vortices calls into question the consistency of mean-flow vorticity monopoles on a closed, spherical manifold, and is addressed using the Circulation Theorem. Vortices with differently shaped wind profiles are also considered to examine effects on waveguide geometry.

Lastly, the VRW paradigm offers insight into analogous, synoptic-scale Rossby Waves in a horizontally sheared flow. Rossby waves propagate within a meridional waveguide confined between a cutoff and zero frequency. A forcing imposed near the middle of a large meridional domain, produces an eastward-propagating wavetrain of comma-cloud-shaped gyres that resemble observed frontal cyclones, whose trailing spirals correspond to the "weathermaker" cold fronts that affect the Southern US. 


\section{TABLE OF CONTENTS}

CHAPTER

PAGE

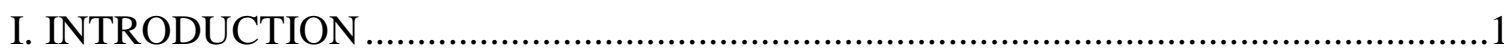

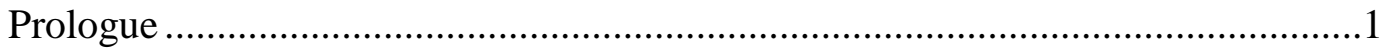

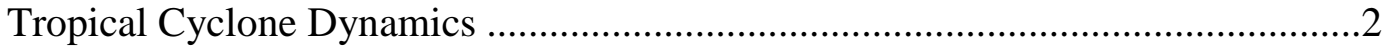

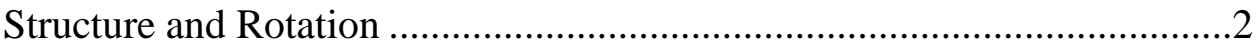

Motion and Vorticity Advection............................................................

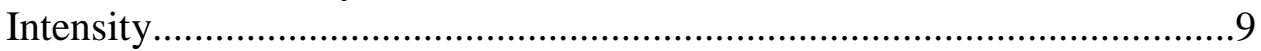

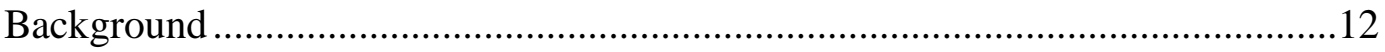

Early VRW History and Theory ………………………......................12

Subsequent VRW Findings .................................................................16

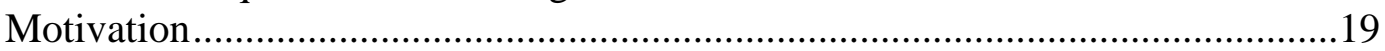

Dissertation Research Questions and Outline ......................................................23

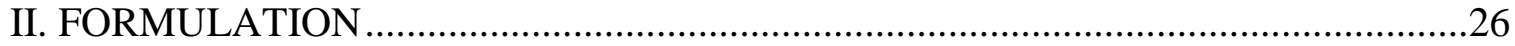

Model Overview and Coordinate System .........................................................26

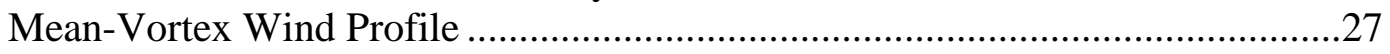

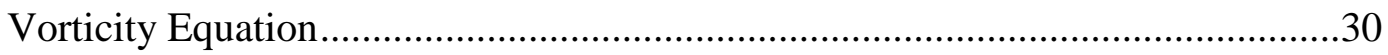

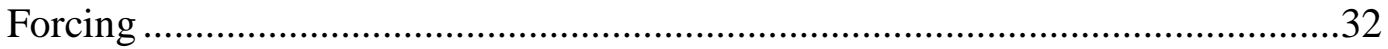

Streamfunction and Geopotential Solutions .....................................................33

Eddy Fluxes and Mean-Flow Acceleration.............................................................36

Wavenumber-1 Nondivergent VRW Dispersion Relation .......................................37

III. WAVENUMBER-1 VRWS AT DIFFERENT SPECIFIED FREQUENCIES ............40

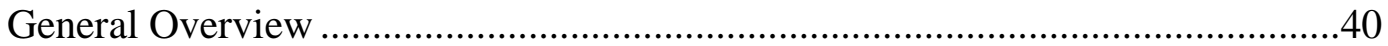

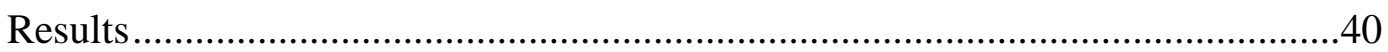

Mean-Vortex Structural Evolution ........................................................40

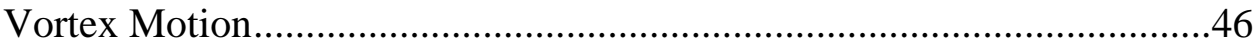

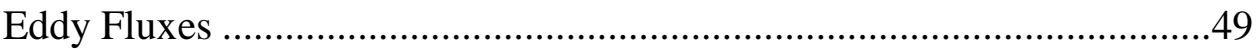

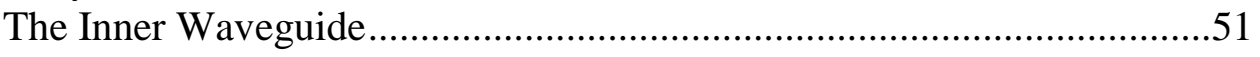

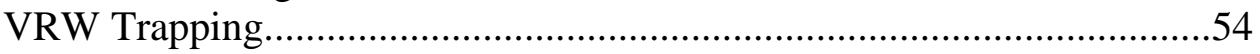

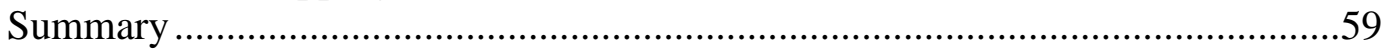

IV. FORCING SENSITIVITY STUDIES AND BETA-PLANE SIMULATIONS .........63

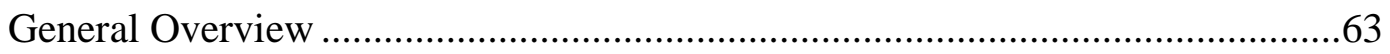

Forcing Sensitivity Studies ...............................................................................64

Forcing Radius and Width ....................................................................64

Model Reinitialization (Episodic Forcing) ………………......................71

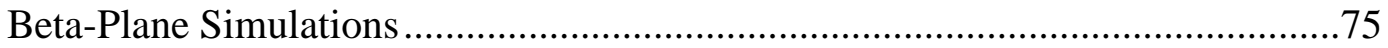

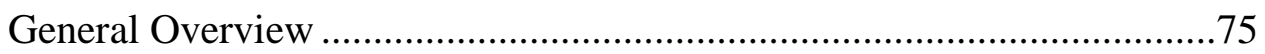

Normal Model Run ..............................................................................

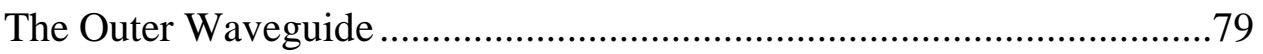

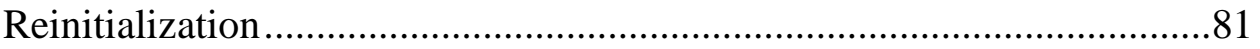




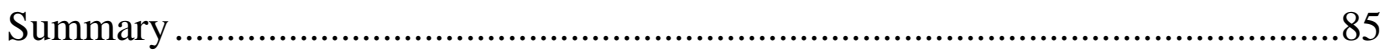

V. VORTEX SENSITIVITY STUDIES AND WIND-PROFILE ANALYSES................89

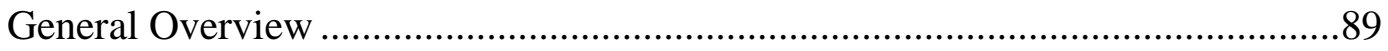

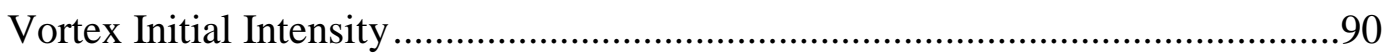

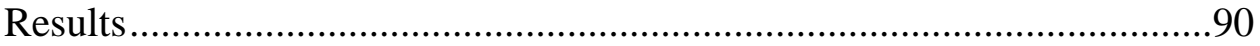

Circulation and Vortical Dynamics ……........................................................98

Stokes' Theorem Applied to Vortices.....................................................98

Constraints on Parametric TC Wind Profiles............................................103

Bounded and Unbounded Vortex Modeling Applications.....................................105

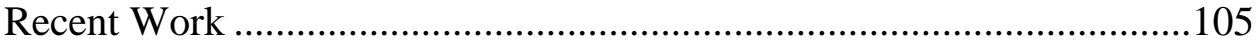

Narrow-Linear-Cubic Profile...............................................................108

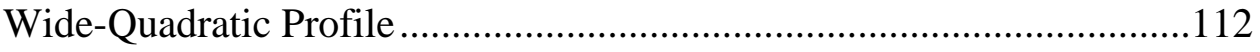

Unbounded Profile ............................................................................114

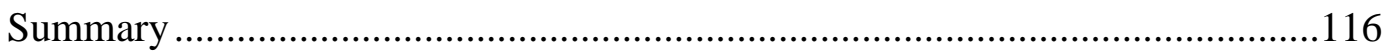

VI. SYNOPTIC-SCALE ROSSBY WAVES IN A MERIDIONALLY SHEARED,

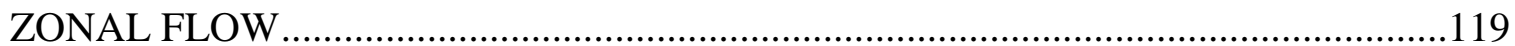

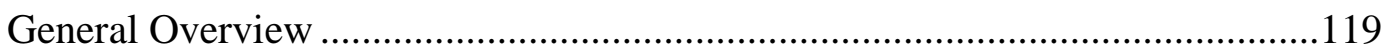

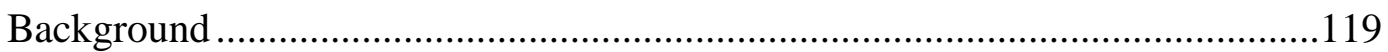

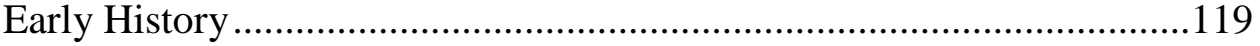

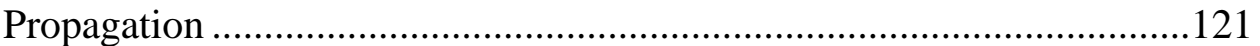

Momentum Transport …………………………………...................121

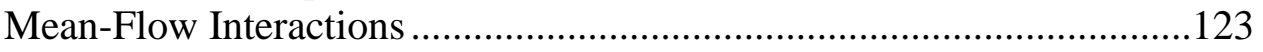

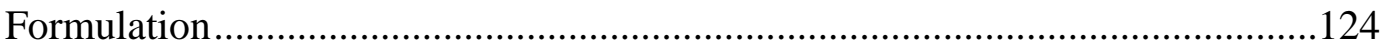

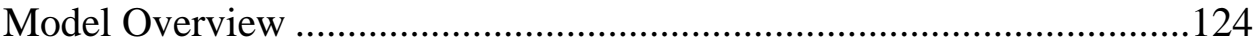

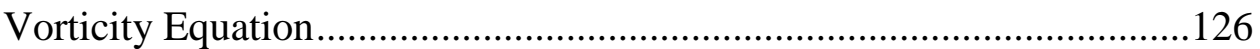

Streamfunction and Geopotential Solutions ...........................................127

Nondivergent Rossby-Wave Dispersion Relation ...................................128

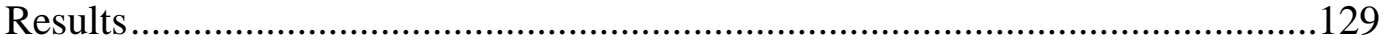

Forced Waves in the Rossby Waveguide............................................129

Eddy Fluxes ………………………………………........................131

Waveguide Sensitivity ........................................................................133

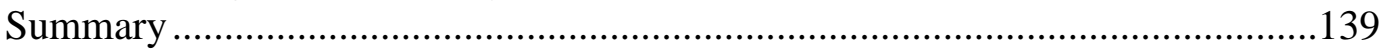

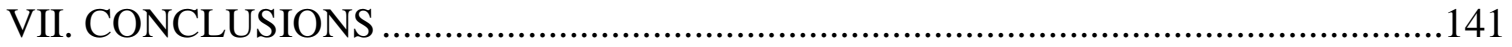

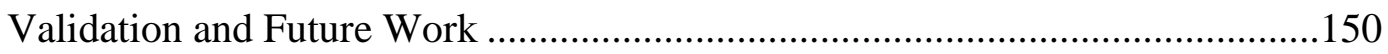

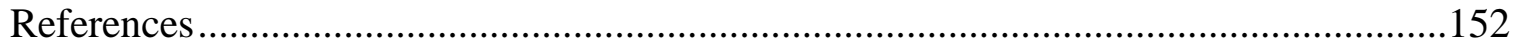

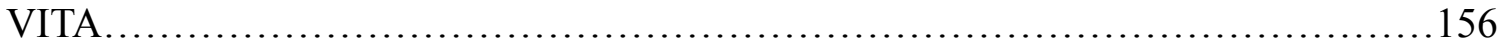




\section{LIST OF TABLES}

TABLE

PAGE

1. Vortex motion results and waveguide width for a forcing with different specified frequencies

2. Wavenumber-1 VRW frequencies at different states

3. Vortex motion and mean-flow acceleration results for sensitivity studies on forcing radius and width

4. Vortex motion results from normal beta-plane simulations versus reinitialized model runs.

5. Vortex parameters, forcing orbital period, and waveguide width for tropical depression, tropical storm, and category-5 mean vortices

6. Vortex motion results for tropical depression, tropical storm, and category-5 mean vortices

7. Waveguide width and vortex-motion results for finitely bounded vortices and an unbounded vortex (3.5-hr orbital period). 


\section{LIST OF FIGURES}

FIGURE

PAGE

Fig 1 - NASA satellite image of Hurricane Irma (2017) ............................................

Fig 2 - Radar image of Hurricane Matthew (2016) showing the heaviest precipitation (warm colors) associated with the outer rainbands and eyewall. 3

Fig 3 - Gradient Balance in a Northern Hemispheric TC: blue arrow is the inward pressure gradient force; green arrow is the outward Centrifugal and Coriolis forces; black arrow is the mean, cyclonic swirling wind (Hurricane Research Division) 4

Fig 4 - Tracks of the Named Storms from the 1999 Atlantic Hurricane Season as an example of most storms recurving out to the open North Atlantic

Fig 5 - Illustration of the beta-drift where the counterflow between the wavenumber-1 asymmetries prompts the vortex to move slowly northwestward (a); hurricane total motion represented as the vector sum between advection by the environmental wind and beta-drift propagation (b)

Fig 6 - An example of observed trochoidal motion from Hurricane Carla (1961) showing multiple track oscillations (roman numerals) occurring periodically on a small-scale

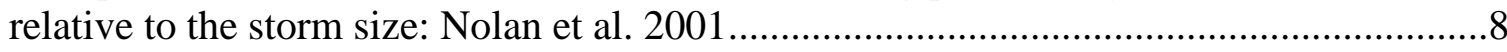

Fig 7 - National Hurricane Center graphic of Hurricane Irma (2017) showing how little wiggles in the track for a landfalling hurricane can lead to significant differences in coastal impacts such as storm surge (Dennis Feltgen).

Fig 8 - Hurricane Olivia radial and tangential profile showing the inverse relationship between wind and pressure: Black et al. 2002

Fig 9 - National Hurricane Center Forecast Intensity Error Trends in the Atlantic Basin, 1985-2017

Fig 10 - Imagery of a TC depicting the outer limits of the spiral bands relative to the storm's axis of rotation (a), compared with a sketch of tilted troughs with respect to Earth's axis of rotation, if viewed from space (b): MacDonald 1968.

Fig 11 - An example of a TC (Hurricane Isabel 2003) displaying eyewall mesovortices: Kossin and Schubert 2004

Fig 12 - Radar reflectivity asymmetries associated with Hurricane Olivia (1994): Reasor et al. 2000

Fig 13 - Wavenumber-2 forcing cycle where the oscillations represent the active 
interval (a) that excites VRWs that manifest as a wavetrain of tear-drop-shaped vorticity gyres advected downstream by the mean-swirling flow with outer trailing spirals (b): Cotto (2012) and Cotto et al. 2015.

Fig 14 - Wavenumber-2 streamfunction (Cotto 2012 and Cotto et al. 2015) whose sunflower-like orientation produces a saddle point in the center (a) that resembles a meteorological col (b)

Fig 15 - Forced waves at $25 \mathrm{~km}$ from the vortex center propagating with a negative Doppler-shifted frequency, $\Omega$ (blue curve) within the inner waveguide bounded by the cutoff frequency, $\Omega_{1 \mathrm{D}}$ (green curve). Area of propagation and loci of energy reflection and absorption are also highlighted: Cotto 2012.

Fig 16 - Vortex Rossby wave dispersion diagram illustrating how the waveguide becomes narrower with increasing tangential wavenumber, and that $\Omega$ approaches zero as radial wavenumber becomes large.

Fig 17 - Mean vortex in a translating cylindrical coordinate system.

Fig 18 - Mean-vortex tangential wind (a), and storm-flow vorticity (b, blue curve) and vorticity gradient profiles (b, orange curve) .

Fig 19 - Forcing contour plot (a) rotating at $25 \%$ of the mean-flow angular velocity (b) at the RMW; 3.5-hr orbital period

Fig 20 - Vorticity contour plots at 8-hr intervals $(\mathrm{a}, \mathrm{b}, \mathrm{c})$ highlighting mean-vortex structural evolution and a comparative radar image of Hurricane Matthew (2016) showing an outer concentric eyewall (d)

Fig 21 - Vorticity contour plots (a) for a 3.5-hr forcing orbital period showing the outer wake aligning with the southeasterly slipstream flow (b)

Fig 22 - Vorticity contour plots for different specified orbital periods: $3.5 \mathrm{hrs}$ (a), $1.8 \mathrm{hrs} \mathrm{(b),} 1.2 \mathrm{hrs} \mathrm{(c),} \mathrm{and} 0.87 \mathrm{hrs} \mathrm{(d)} \mathrm{showing} \mathrm{symmetrized} \mathrm{trailing} \mathrm{spirals} \mathrm{at} \mathrm{the} \mathrm{end}$ of each simulation

Fig 23 - Vorticity radial structure for different specified orbital periods: 3.5 hrs (a), 1.8 hrs (b), $1.2 \mathrm{hrs}$ (c), and $0.87 \mathrm{hrs}(\mathrm{d})$, with anomalies highlighting the locus of forcing, propagating VRWs, and critical radius

Fig 24 - Streamfunction contour plots for different specified orbital periods: $3.5 \mathrm{hrs}$ (a), $1.8 \mathrm{hrs}(\mathrm{b}), 1.2 \mathrm{hrs}(\mathrm{c})$, and $0.87 \mathrm{hrs}$ (d) highlighting the nondivergent flow

Fig 25 - Vortex tracks showing a cyclonic orbit for different specified orbital periods:

$3.5 \mathrm{hrs}(\mathrm{a}), 1.8 \mathrm{hrs}(\mathrm{b}), 1.2 \mathrm{hrs}$ (c), and $0.87 \mathrm{hrs}(\mathrm{d})$ 
Fig 26 - Vortex orbital speeds ranging from $\sim 2.25$ to $3.6 \mathrm{~ms}^{-1}$ for different forcing orbital periods (a), and direction of motion for a 3.5-hr orbital period (b). The latter shows that the vortex center completes nearly 7 orbits

Fig 27 - Angular momentum (blue curve) and geopotential fluxes (orange curve) for different specified orbital periods: $3.5 \mathrm{hrs}$ (a), $1.8 \mathrm{hrs}$ (b), $1.2 \mathrm{hrs}$ (c), and $0.87 \mathrm{hrs}$ (d) ...50

Fig 28 - Mean-flow acceleration (orange curve) superimposed with angular momentum flux (blue curve) for different specified orbital periods: $3.5 \mathrm{hrs}$ (a), $1.8 \mathrm{hrs}$ (b), $1.2 \mathrm{hrs}$ (c), and $0.87 \mathrm{hrs}(\mathrm{d})$

Fig 29 - Wavenumber-1 inner waveguide (top row) highlighting that frequency passbands between the cutoff (red curve) and zero frequency (blue curve) represent free-propagating VRWs (a) radially trapped VRWs Doppler-shifted to the cutoff frequency twice (b). Wavenumber-2 (c) and wavenumber-3 (d) inner waveguides are much narrower by comparison.

Fig 30 - Vorticity (a,b) and streamfunction contour plots (c,d) for a 14.5-hr orbital period (left column) and a -3.5 -hr orbital period (right column).

Fig 31 - Vortex tracks (left column); orbital speed (right column); Top row: 14.5-hr forcing orbital period; bottom row: -3.5 -hr forcing orbital period

Fig 32 - Radial fluxes $(\mathrm{a}, \mathrm{c})$ and mean-flow acceleration induced by angular momentum flux (b,d) for a 14.5-hr orbital period (top row) and a -3.5 -hr orbital period (bottom row)..

Fig 33 - Forcing $(a, b)$ and vorticity $(c, d)$ contour plots with comparative images of Hurricane Matthew, 2016 (e) and Hurricane Juliette, 2001 (f) for a 15-km forcing radius (left column) and $35-\mathrm{km}$ forcing radius (right column)

Fig 34 - Vortex track (a,b), orbital speed (c,d), and mean-flow acceleration induced by angular momentum fluxes (e,f) for a $15-\mathrm{km}$ forcing radius (left column) and a $35-\mathrm{km}$ forcing radius (right column)

Fig 35 - Forcing (a,b), vorticity (c,d), and streamfunction (e,f) contour plots for a 5-km forcing width (left column) and a $20-\mathrm{km}$ forcing width (right column).

Fig 36 - Vortex track (a,b), orbital speed (c,d), and mean-flow acceleration induced by angular momentum fluxes (e,f) for a 5-km forcing width (left column) and a $20-\mathrm{km}$ forcing width (right column).

Fig 37 - Vorticity contour plots before (left column) and after model reinitialization 
(right column) for when the forcing amplitude is halved (b), reduced to zero (d), and doubled (f)

Fig 38 - Vortex track (left column) and orbital speed (right column) when forcing amplitude is halved $(a, b)$, reduced to zero $(c, d)$, and doubled $(e, f)$

Fig 39 - Complete nonlinear solution for streamfunction from GCW's barotropic nondivergent model on a beta plane illustrating the linearly forced wavenumber-1 beta gyres (a), nonlinearly forced wavenumber-1 anti-beta gyres (b), and nonlinearly forced wavenumber-2 gyres (c)

Fig 40 - Beta-gyre vorticity (a) and streamfunction (b) dipole, with induced trochoidal track (c) and translation speed (d)....

Fig 41 - Mean-vortex radial profile extending out to $3000 \mathrm{~km}$, showing reversal of mean-flow relative vorticity, becoming anticyclonic on the periphery before asymptoting to zero (a); corresponding outer waveguide supporting propagation of only very low-frequency waves upon the weakly positive radial vorticity gradient (b)....

Fig 42 - Beta-gyre streamfunction dipole before (a) and after (b) reinitialization

Fig 43 - Vortex track (left column) and translation speed (right column) for zero beta $(a, b)$, zero convective forcing $(c, d)$, and total forcing reduced to zero $(e, f)$.

Fig 44 - TD-vortex radial profiles (top row), forced wavenumber-1 dipole (mid row), and vortex motion (bottom row)

Fig 45 - TS-vortex radial profiles (top row), forced wavenumber-1 dipole (mid row), and vortex motion (bottom row)

Fig 46 - CAT5-vortex radial profiles (top row), forced wavenumber-1 dipole (mid row), and vortex motion (bottom row)

Fig 47 - Radial wind and vorticity profile for the LN3 (a), W2 (b), and unbounded (c) vortices with $50 \mathrm{~ms}^{-1}$ max winds at $25 \mathrm{~km}$.

Fig 48 - Illustration of Stokes' Theorem: Davis (1961)

Fig 49 - A free-slip boundary condition in a 3D atmosphere with no rigid lid, where vortex tubes (black arrows) rise in the vortex's eyewall to produce cyclonic vertical vorticity (red arrows) in the core, spread at tropopause level, and then subside to produce anticyclonic vorticity and terminate at the boundary-layer surface (a). An example of vortex tube surface termination (b) 
Fig 50 - A no-slip boundary condition where the rising vortex tubes reconnect at the vortex periphery

Fig 51 - Illustration of a cyclonic vorticity patch on a sphere with no vorticity anywhere else and the apparent anticyclonic circulation at the patch's antipode, but with no enclosed vorticity

Fig 52 - Integrating from the center of the cyclonic vorticity patch outward to the boundary and cross-equatorially toward the antipode which results in a singularity where the wind must approach infinity to keep the circulation constant.

Fig 53 - Beta-gyre vorticity asymmetries (upper left) for an asymptotically bounded and unbounded vortex $(\mathrm{a}, \mathrm{b})$ and two finitely bounded vortices $(\mathrm{c}, \mathrm{d})$; northwestward beta-drift for each vortex (upper right); radial vorticity gradient for the finitely bounded vortices (bottom row): Gonzalez et al. 2015

Fig 54 - LN3 vortex radial profiles (top row), forced wavenumber-1 dipole (mid row), and vortex motion (bottom row)

Fig 55 - W2 vortex radial profiles (top row), forced wavenumber-1 dipole (mid row), and vortex motion (bottom row)

Fig 56 - Unbounded vortex radial profiles (top row), forced wavenumber-1 dipole (mid row), and vortex motion (bottom row).

Fig 57 - Carl-Gustaf Rossby and an interpretation of his waves with respect to the Earth's circumpolar winds and the jet stream: Segund (1956)

Fig 58 - Meridional profiles for mean flow (a), planetary vorticity (b), and beta (c)......125

Fig 59 - Imposed forcing's meridional structure (a) and contour plot (b) near the middle of the domain

Fig 60 - Streamfunction (a) depicting a wavetrain of comma-cloud-shaped gyres within an extensive meridional waveguide (b) that resembles observed frontal cyclones on satellite (c) and surface weather maps (d, source: Weather Prediction Center)

Fig 61 - Horizontal fluxes of geopotential and momentum, showing that the forced Rossby waves transport westerly momentum poleward (a) and energy equatorward (b).

Fig 62 - Mean-flow profile and forced wavetrain in pure westerly flow (top row), most realistic flow (mid row), and in pure easterly flow (bottom row) 134 
Fig 63 - Mean-flow profile $(\mathrm{a}, \mathrm{b})$ and forced wavetrain $(\mathrm{c}, \mathrm{d})$ for a shear value:

$1.25 \times 10^{-3} \mathrm{~s}^{-1}$ (left column); and shear value: $5 \times 10^{-3} \mathrm{~s}^{-1}$ (right column) 136

Fig 64 - Forcing contour plot and forced wavetrain for a 3000-km forcing latitude (top row), 2000-km forcing latitude (mid row), and a 1000-km forcing latitude (bottom row) 


\section{ABBREVIATIONS AND ACRONYMS}

Asymmetric Balance

$\mathrm{AB}$

Barotropic, nondivergent

BND

Category 5

CAT5

Cotto et al. (2015)

CGW

Gonzalez et al. (2015)

GCW

Narrow-linear cubic

LN3

Montgomery and Kallenbach (1997)

MK97

Nolan and Montgomery (1999)

NM99

Tropical cyclone

TC

Tropical depression

TD

Tropical storm

TS

Vortex Rossby wave

VRW

Willoughby (1992)

W92

Wood-White

WW 


\section{CHAPTER I. INTRODUCTION}

Prologue

The destructive power of hurricanes and typhoons (i.e., Tropical Cyclones, TCs) poses persistent worldwide threats to life, property, and the economy. Tropical Cyclones kill hundreds of people, and cause tens to hundreds of billions of dollars in damage annually. Moreover, the magnitude of TC impacts is increasing as coastal populations and development continue to grow. An additional concern is the potential for highfrequency devastating storms in response to climate change. Global Climate Models suggest a decline in TC frequency on a warmer planet, but also predict that the strongest storms will become stronger (Knutson et al. 2010, Landsea 2005, Pielke, Jr. et al. 2005 and 2006). If the models' climatological forecasts verify, seasons with intense TCs may become common. It is therefore urgent to further improve upon understanding of TC dynamics, particularly inner-core asymmetric perturbations. The main goal of this dissertation is modeling vortex Rossby waves (VRWs) to advance physical understanding of the waves' roles in TC motion and VRW propagation within the waveguide, and clarify the waves' impacts on structure and mean-flow distribution. In addition, model sensitivity studies are conducted and the model framework is extended to mid-latitude Rossby waves. The rest of Chapter 1 reviews TC dynamics, mostly in the context of VRWs, presents relevant background studies, discusses research motivation, and concludes with an outline of subsequent chapters. 
Tropical Cyclone Dynamics

Structure and Rotation

Tropical cyclones are axially symmetric, rapidly rotating, warm-core vortices that evolve slowly over time. A mature TC's typical satellite presentation is a rotating disk of intense convection with a well-defined, (usually) cloud-free eye in the center, surrounded by the Central Dense Overcast of very cold cloud tops and high cirrus outflow (Fig 1). On radar imagery, the eyewall contains the heaviest precipitation surrounded by outer, cyclonically (counterclockwise in Northern Hemisphere) curved rainbands embedded in less intense, stratiform precipitation (Fig 2). Between some rainbands are "moat" regions of minimal precipitation that coincide with sinking motion. It is shown later that VRWs play a role in the horizontal processes that produce spiral bands. Spiral rainbands are significant features for the following reasons: 1) Rainbands make up a large portion of TC structure; 2) Although the clouds associated with spiral rainbands are primarily driven by vertical convective processes, the clouds' non-uniform distribution implies that horizontal asymmetries in the vertical motion also play an important role; 3) MacDonald (1968) noted that early radar observations showed consistently stronger surface winds under rainbands in landfalling, New England Hurricanes Edna (1954) and Esther (1962). 


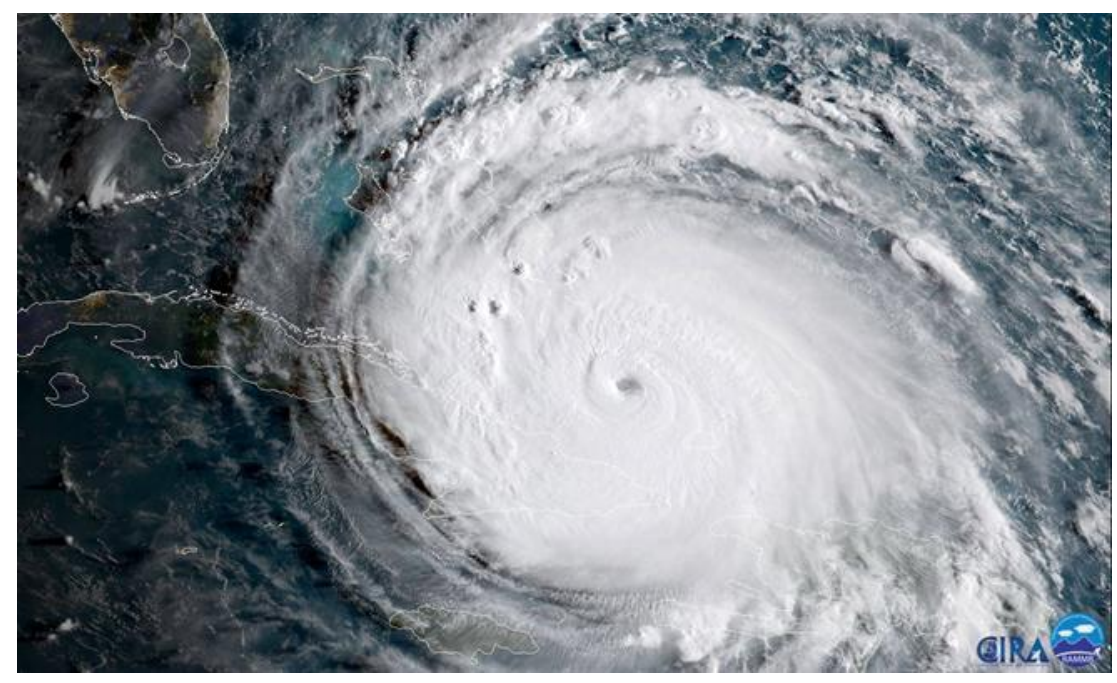

Fig 1. NASA satellite image of Hurricane Irma (2017).

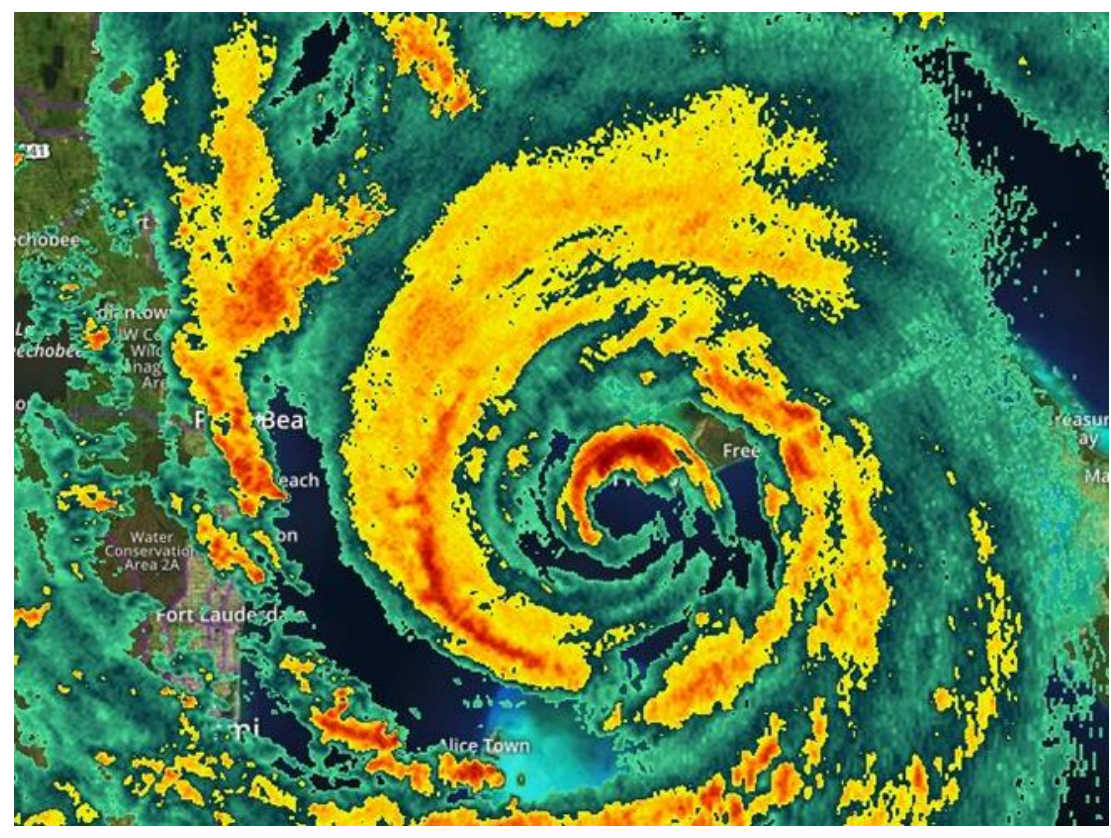

Fig 2 - Radar image of Hurricane Matthew (2016) showing the heaviest precipitation (warm colors) associated with the outer rainbands and eyewall.

The primary circulation of a TC consists of cyclonically swirling flow around and spiraling into the low-pressure center in approximate Gradient Balance between inwardly directed pressure-gradient force $\left(\rho^{-1} \partial p \partial r\right)$, and the outwardly directed centrifugal $\left(v^{2} / r\right)$ 
and Coriolis $(f v)$ apparent forces. In the Northern Hemisphere, the inertial forces act orthogonally to the right of the wind to produce the counterclockwise flow (Fig 3). The small, but rapidly rotating inner cores of a TC however are closer to Cyclostrophic Balance, where $f v$ is small so that, $v_{\mathrm{c}}^{2} / r=\rho^{-1} \partial p \partial r$, where $v_{c}$ is the cyclostrophic wind. Gradient and cyclostrophic wind play important roles in determining the wind profiles of TC-like vortices (discussed in Chapter 2).

\section{Gradient Balance}

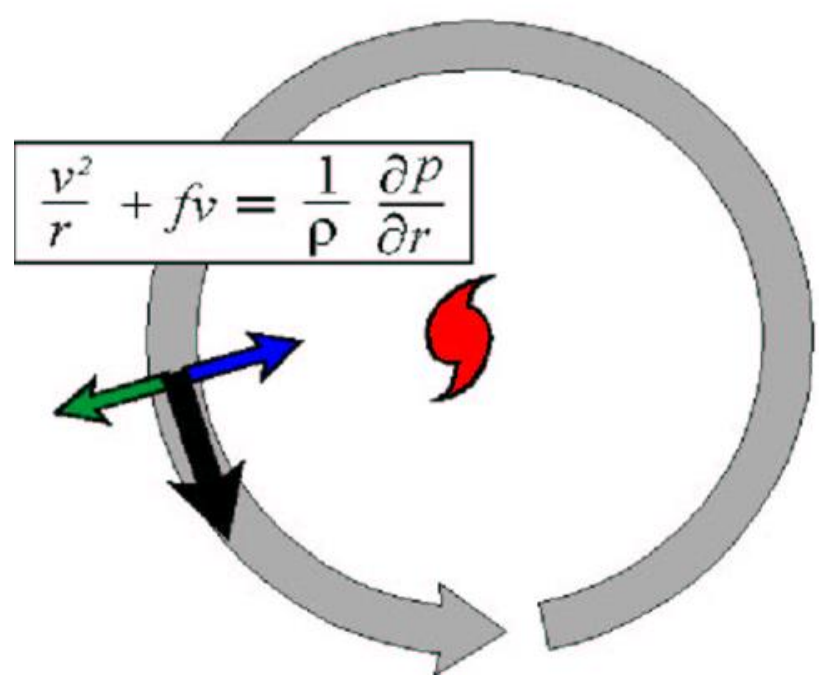

Fig 3. Gradient Balance in a Northern Hemispheric TC: blue arrow is the inward pressure gradient force; green arrow is the outward Centrifugal and Coriolis forces; black arrow is the mean, cyclonic swirling wind (Hurricane Research Division).

Since TCs are overwhelmingly rotational, vorticity is a key dynamical variable. Relative vorticity, $\zeta$, is the vorticity relative to the Earth, defined as the vector curl of the horizontal wind. Typical radial TC profiles show strongly positive (cyclonic) $\zeta$ near the eye where the rotation is strongest, with a monotonic decay with distance outward from the center. Depending on the properties of the wind profile, either an annulus of negative 
(anticyclonic) $\zeta$ or irrotational flow such that, $\zeta=0$ can exist far from the vortex center (discussed further in Chapter 5). Regardless, there is a negative radial vorticity gradient within most of the vortex core that primarily defines the VRW inner waveguide (Cotto et al. 2015, hereafter CGW). Therefore VRWs are considered rotational, vorticity waves. The next subsection reviews how the large-scale atmosphere, vorticity advection, and convection influence TC motion. The last two are of most interest.

\section{Motion and Vorticity Advection}

Tropical cyclone motion is generally governed by the large-scale atmospheric steering flow. In the subtropical Atlantic basin, most TCs are steered east-to-west by the easterly flow on the southern periphery of the Bermuda-Azores High (Fig 4). At the western periphery, many storms' forward motion briefly slows before recurvature into the open North Atlantic, followed by eventual dissipation over cold water. Another mechanism that contributes to TC motion is the beta ( $\beta$ ) effect (e.g., Holland 1983, and Chan and Williams 1987) which is determined by vorticity dynamics. In meteorology, a $\beta$-plane represents the meridional gradient of planetary vorticity, $f$, defined as the component of the Earth's rotation about the local vertical, such that $\beta=$ $\partial f / \partial y=\left(2 \Omega_{0} / R_{E}\right) \cos \varphi$ is locally constant. Here, $y$ is the meridional distance, $\varphi$ is latitude, and $\Omega_{\mathrm{o}}$ and $R_{E}$ are the Earth's angular velocity and radius respectively. By contrast, an $f$ plane neglects the $\beta$-effect by assuming constant $f$. Absolute vorticity $(\zeta+f)$ conservation is essential to TC motion on a $\beta$-plane. In order for a poleward-displaced, rotating air parcel to conserve absolute vorticity, $\zeta$ must decrease as $f$ increases. 


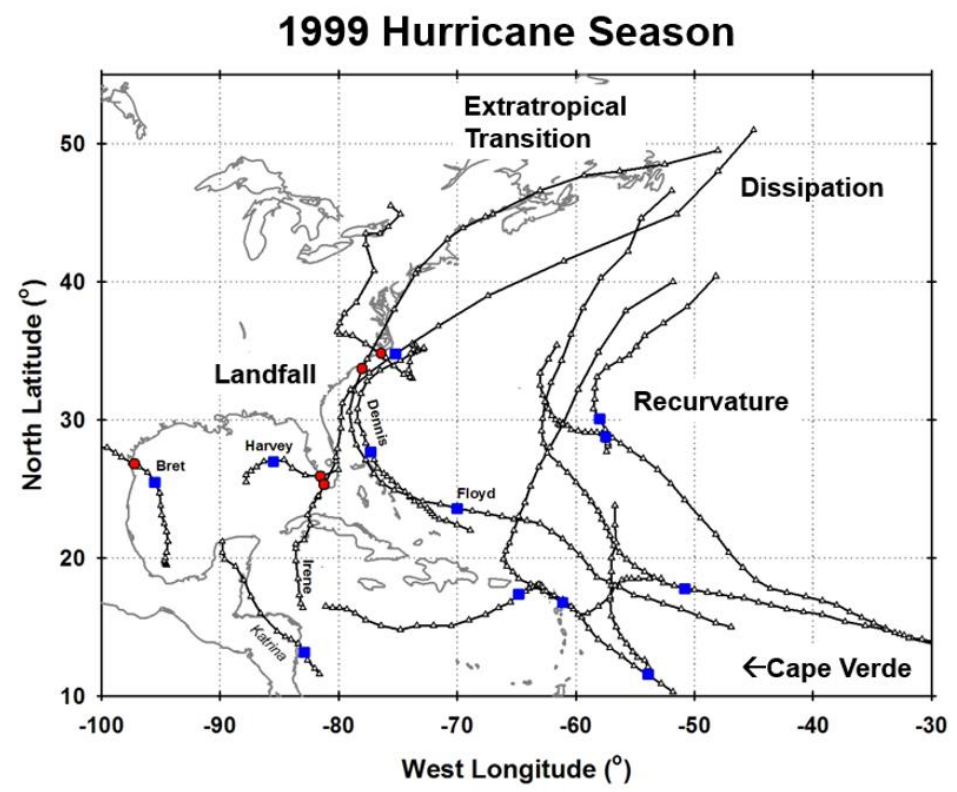

Fig 4. Tracks of the Named Storms from the 1999 Atlantic Hurricane Season as an example of most storms recurving out to the open North Atlantic.

A TC's cyclonic flow approximately conserves absolute vorticity as storm-relative vorticity is advected around the center. The north side of a TC's circulation is at higher latitudes so that air swirling around the eastern periphery encounters larger $f$ and acquires decreased $\zeta$ to produce an anticyclonic gyre to the north. Conversely, air rounding the western periphery experiences decreasing $f$ and increasing $\zeta$, producing a cyclonic gyre to the south. Between the north-south oriented gyres the vorticity dipole tilts to produce a "ventilation flow" across the TC center that advects the axially symmetric vorticity westward. Secondly, the environmental air comes under the influence of the storm's rotation which increases $\zeta$ to the west and decreases $\zeta$ to the east (Chan and Williams 1987, and Marks 1992). Therefore a cyclonic (anticyclonic) gyre forms on the western (eastern) periphery to produce a northward counterflow. In the Northern Hemisphere, the combination of the westward and northward flows from both pairs of gyres yields a 
consensus vortex translation to the northwest at $\sim 1-2 \mathrm{~ms}^{-1}$, known as the $\beta$ drift (Fig 5a; revisited in Chapter 4). Total TC motion is thus the vector sum of advection by the largescale steering flow, and $\beta$-drift propagation (Fig 5b) in the Beta and Advection Model used by the National Hurricane Center to aid in track forecasting.
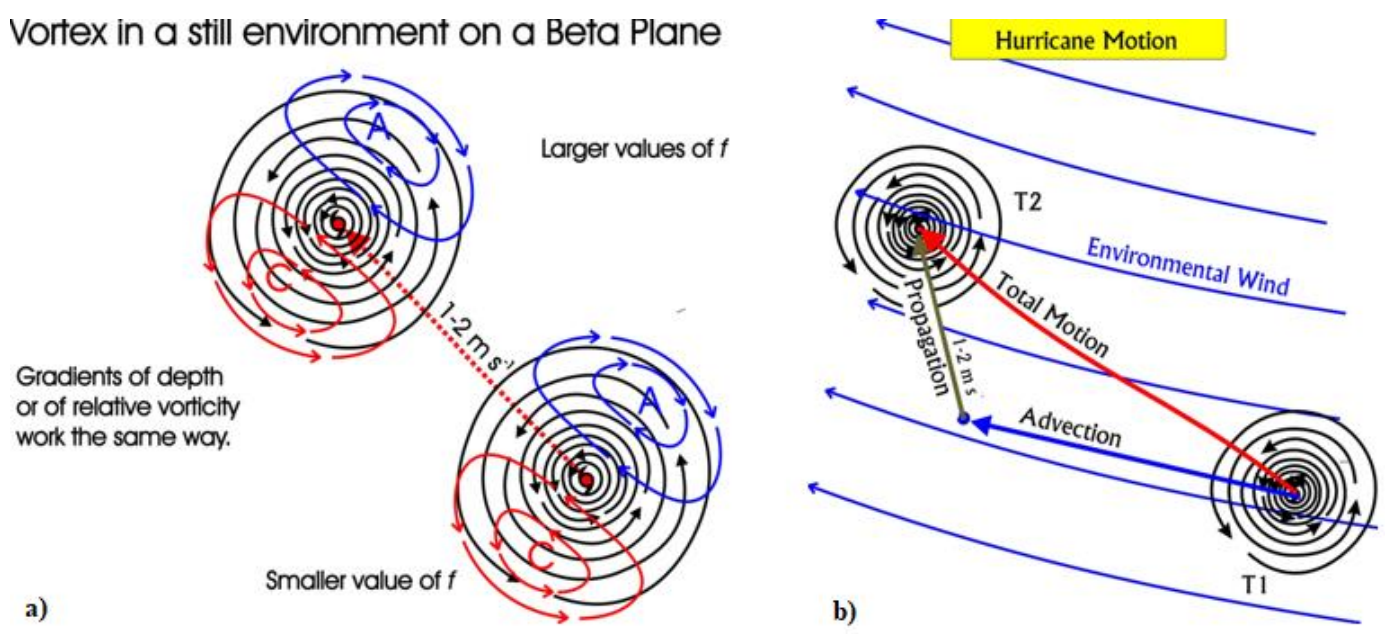

Fig 5. Illustration of the beta-drift where the counterflow between the wavenumber-1 asymmetries prompts the vortex to move slowly northwestward (a); hurricane total motion represented as the vector sum between advection by the environmental wind and beta-drift propagation (b).

Convective bursts that often occur within TCs can also induce motion by drawing the center toward the locus of convection. Convective asymmetries are more complicated than the static $\beta$-gyres because the cells rotate rapidly around the center, which causes the vorticity to be advected quick in non-uniform directions, therefore contributing to observed trochoidal motion (Fig 6; e.g., Nolan and Montgomery 1999, Nolan et al. 2001, and Lawrence and Mayfield 1977). Moreover, convective cells embedded in a TC can change the vorticity through strongly divergent flow (Willoughby 1992, hereafter W92). Updrafts produce clouds and act to maintain mass conservation. 
In the hurricane surface boundary layer, frictional inflow concentrates horizontal vortex tubes toward the center that are titled upward by the strong eyewall updrafts. The tubes are stretched by accelerating updrafts to create vorticity sources and sinks that can cause small-scale, vortex trochoidal motion.

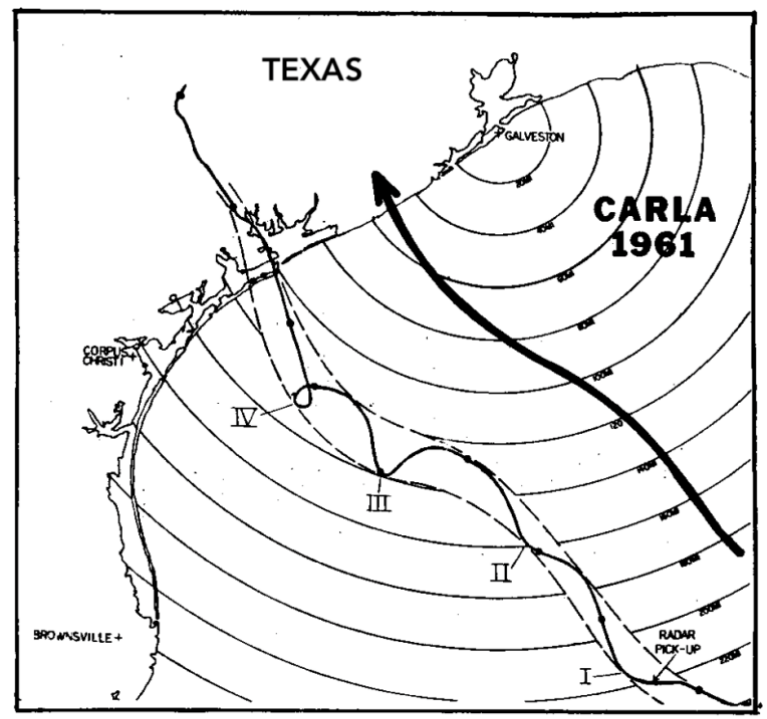

The path of Hurricane Carla (1961) as reanalyzed by Jarvinen et al. (1984), showing four trochoidal oscillations just before landfall. Also shown (thick line) is the "best track path," displaced northward for comparison. The range markers are at $20 \mathrm{n}$ mi intervals. Original figure was adapted from Weatherwise, Oct 1961.

Fig 6. An example of observed trochoidal motion from Hurricane Carla (1961) showing multiple track oscillations (roman numerals) occurring periodically on a small-scale relative to the storm size: Nolan et al. 2001.

Chapter 3 shows that a wavenumber-1 rotating mass source-sink pair in a simulated TC's eyewall generates VRWs that propagate radially away from the locus of excitation, and produces trochoidal vortex tracks. Though smoothed out by forecasters when constructing a TC's best track, trochoidal motion becomes a potentially important factor when a storm is threatening land. Even slight track deviations can sometimes make significant differences on coastal impacts. A recent example was Hurricane Irma (2017), 
whose wiggle to the east spared the Florida coastline from Naples northward to Cape Coral from storm surge inundation greater than nine feet (Fig 7). Could VRWs have contributed to the eastward deviation from the forecast track?
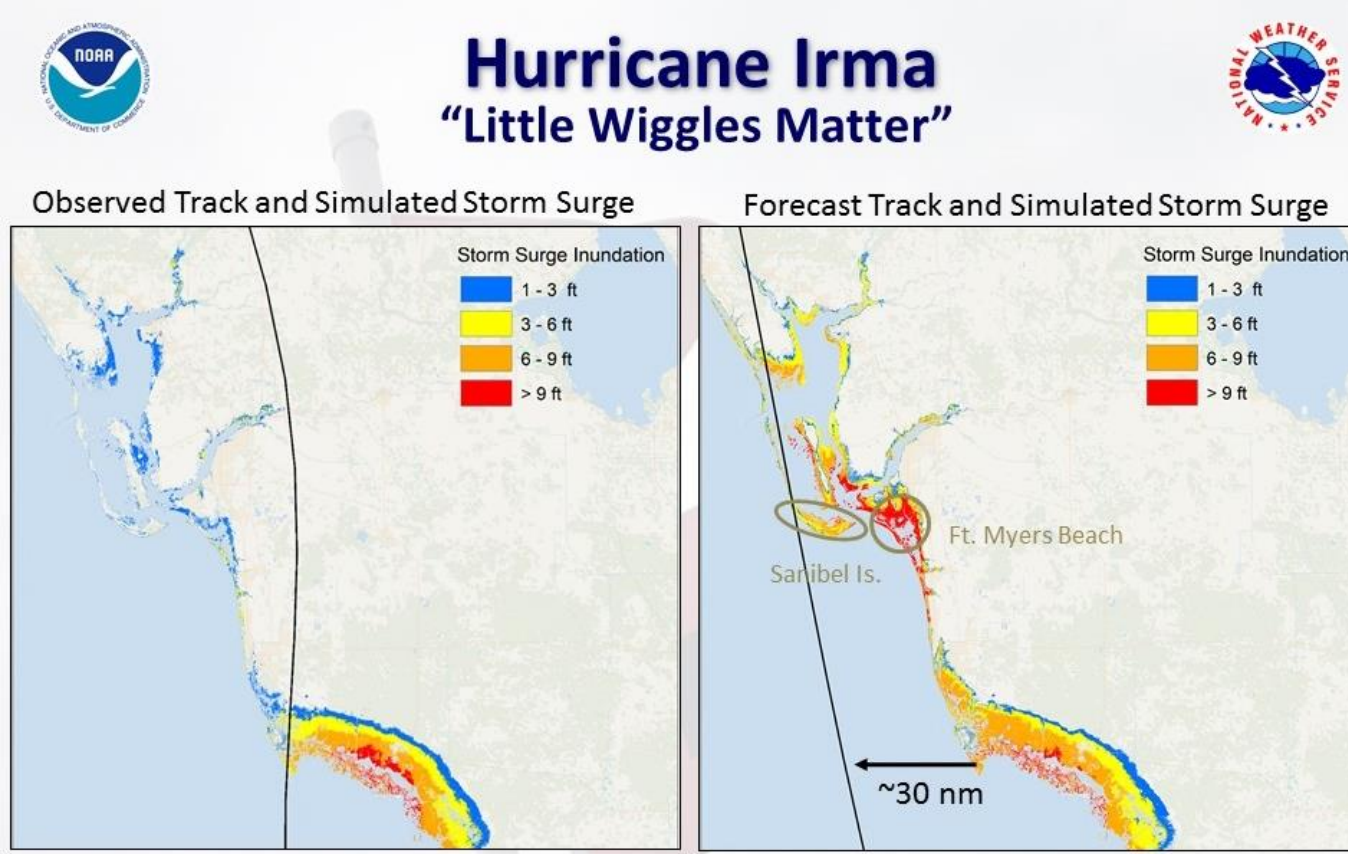

$\sim 50,000$ people with $3+$ foot surge Forecast Track and Simulated Storm Surge

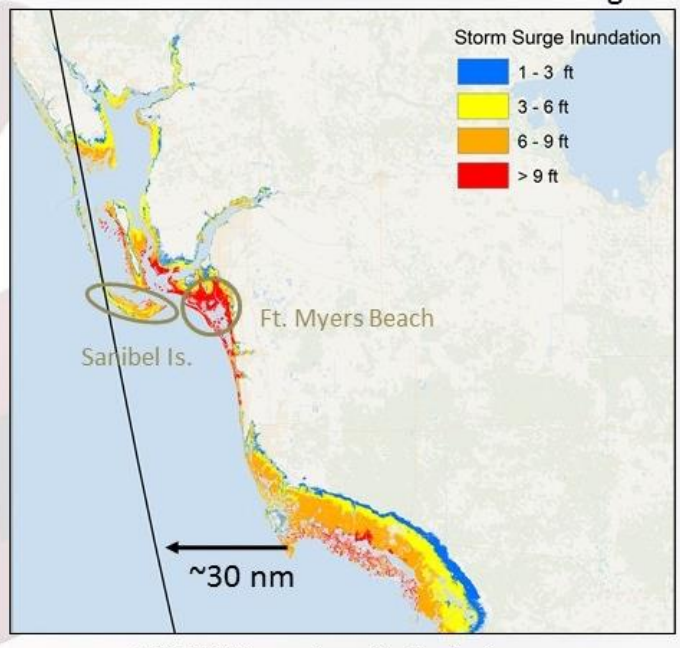

$\sim 200,000$ people with $3+$ foot surge

Fig 7. National Hurricane Center graphic of Hurricane Irma (2017) showing how little wiggles in the track for a landfalling hurricane can lead to significant differences in coastal impacts such as storm surge: Dennis Feltgen.

Intensity

Tropical cyclone intensity is quantified by its maximum sustained winds. Hurricanes, typhoons, and cyclones are ranked on the Saffir-Simpson Scale into Categories 1 through 5. Category- 1 storms must have $\geq 65-\mathrm{kt}$ maximum sustained winds and major hurricanes/super typhoons are defined by Category 3 ( $\geq 96 \mathrm{kts})$. Minimum central pressure in the eye is also an indicator of intensity. Wind and pressure have inverse variation (Fig 
8). The strong pressure gradients between the TC periphery and center sustain the powerful winds in approximate gradient balance and frictional inward convergence of air toward the eyewall. Winds are strongest in the eyewall and generally decrease with increasing radius outward from the eye. The eye is calm at the center because of strong sinking motion that inhibits convection. Eyewall convection that is modeled to excite VRWs, rotates with a frequency that is represented in terms of the TC's mean-flow angular velocity.

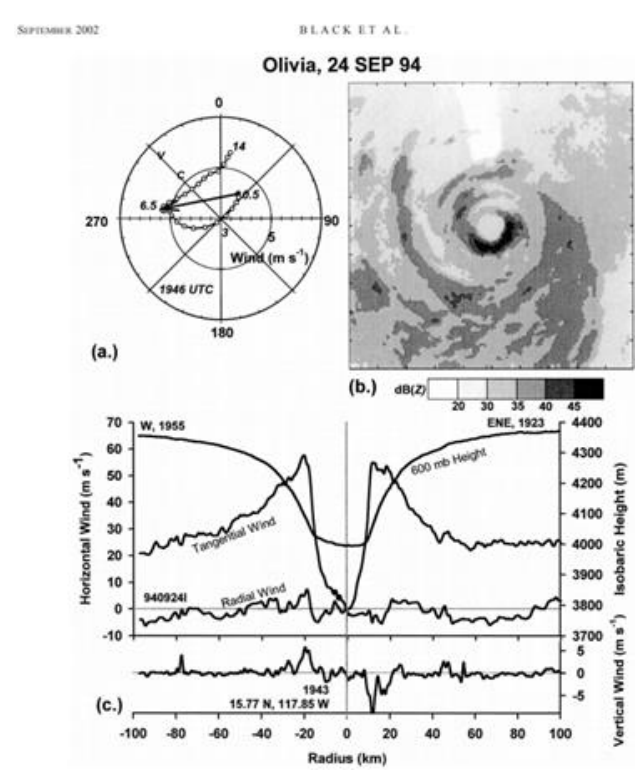

Fig 8. Hurricane Olivia radial and tangential profile showing the inverse relationship between wind and pressure: Black et al. 2002.

Environmental conditions are relatively simple to interpret in order to generally gauge how a TC's future intensity may change. However, complex inner-core dynamics can also profoundly influence a storm's structure and strength, which contribute to intensity forecast errors (Fig 9). Vortex Rossby waves have been suggested to influence TC intensity by accelerating the mean flow near the storm's eyewall or altering the eyewall shape. However, TC intensity can also impact VRW propagation (shown in 
Chapter 5). The next section discusses VRW history and how subsequent theoretical studies have contributed to the understanding of the waves' influences, including observational validation.

NHC 12-120h Intensity Forecast Errors

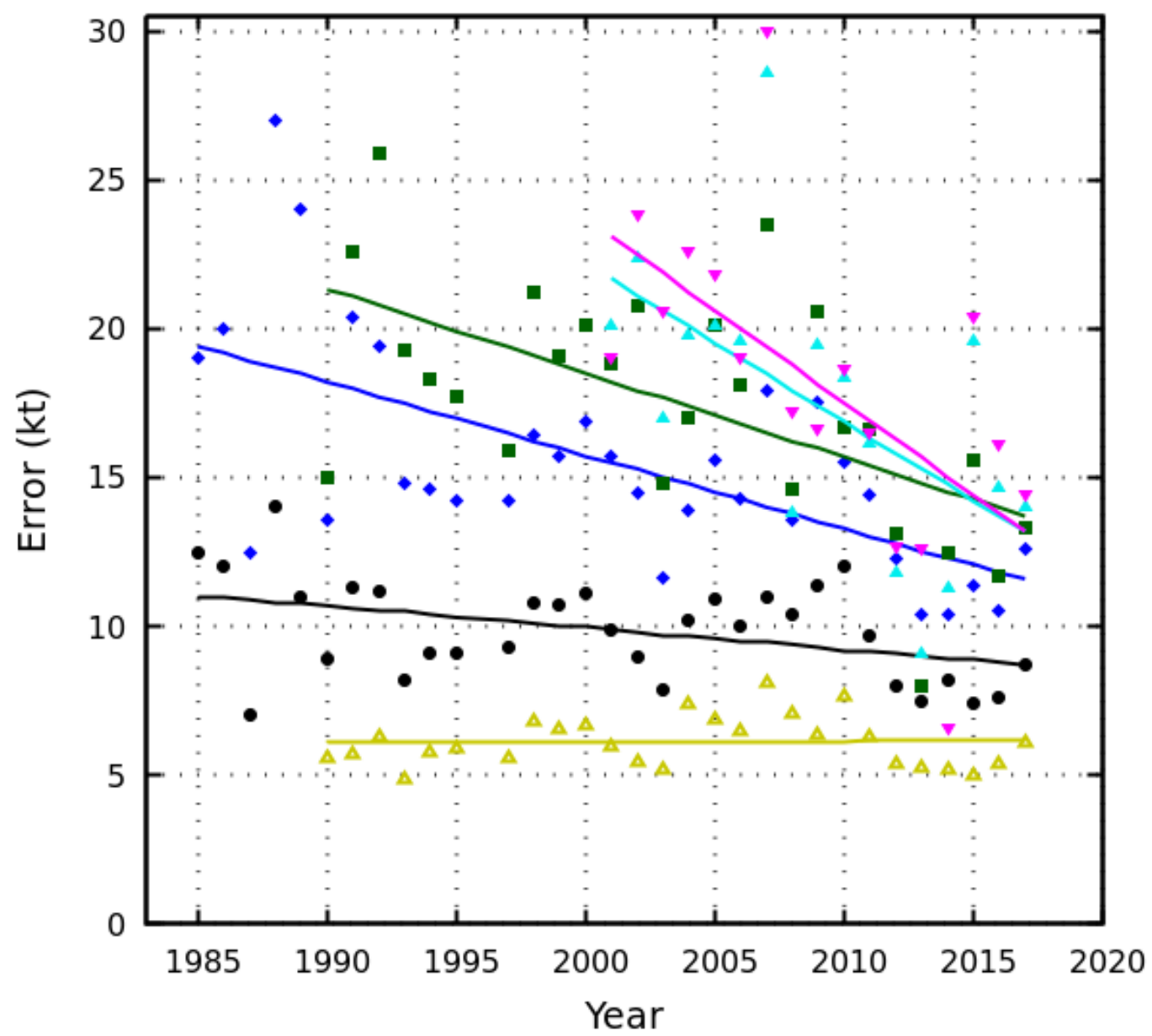

Fig 9. National Hurricane Center Forecast Intensity Error Trends in the Atlantic Basin, 1985-2017. 


\section{Background}

\section{Early VRW History and Theory}

The existence of VRWs was first recognized when observed hurricane rainbands were carefully studied by MacDonald (1968, Fig 10a) and exhibited both qualitative and quantitative, Rossby-wave-like characteristics. At the time, Rossby waves were understood as organized eddy structures in the Earth's synoptic-scale atmosphere that depend upon the variation with latitude of the vertical component of the Earth's rotation (discussed further in Chapter 6). MacDonald noticed that the spiral bands tilted outward from a TC's axis of rotation, similar to the way that synoptic-scale troughs do (Fig 10b). In both cases, the "trains" move with the mean flow, but more slowly. Lastly, inward transport of cyclonic angular momentum within TCs is analogous to the poleward flux of westerly angular momentum in the general circulation. Horizontal eddy exchange processes are responsible for both angular momentum transport mechanisms. MacDonald's analogies ultimately inspired the development of a formal theory on VRW dynamics by Montgomery and Kallenbach (1997, hereafter MK97) that laid the foundation for subsequent VRW modeling efforts. 

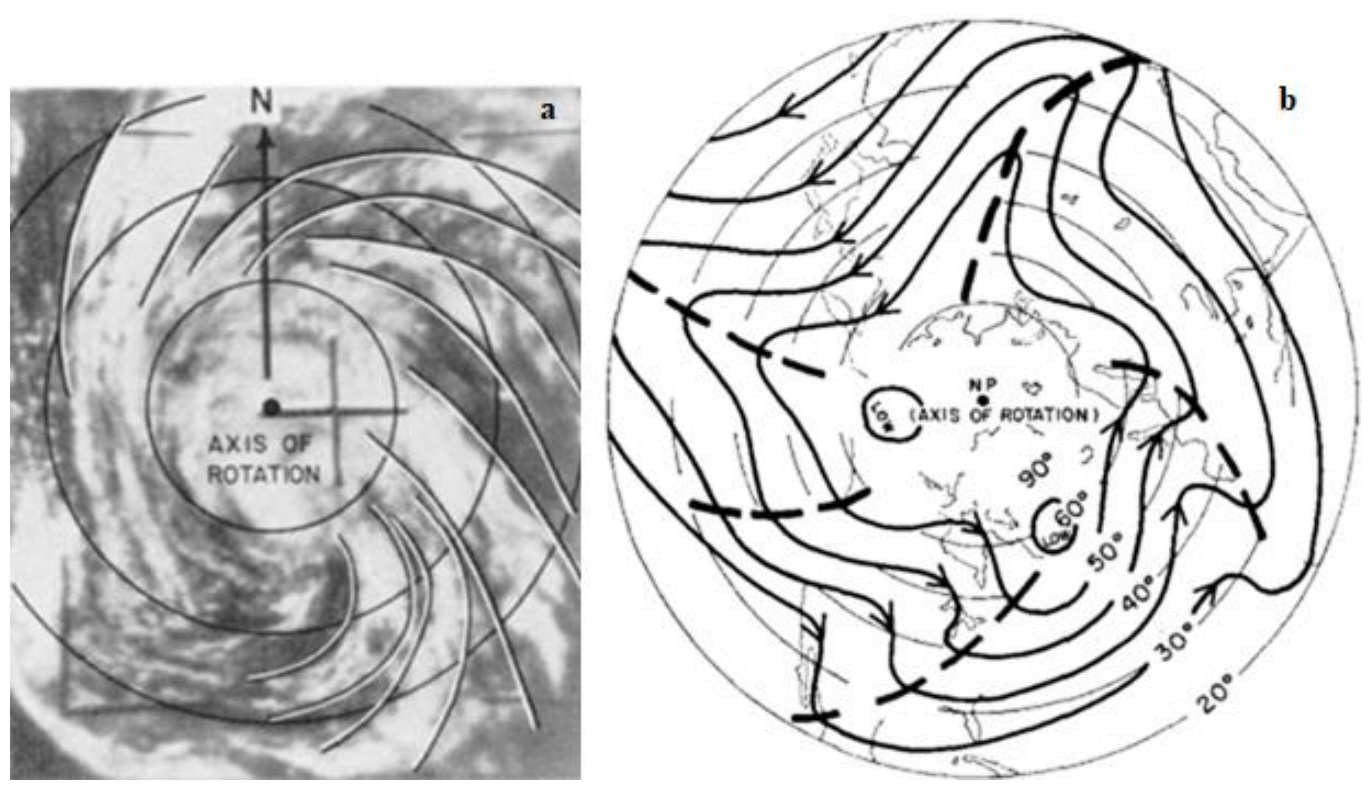

Fig 10. Imagery of a TC depicting the outer limits of the spiral bands relative to the storm's axis of rotation (a), compared with a sketch of tilted troughs with respect to Earth's axis of rotation, if viewed from space (b): MacDonald 1968.

An Asymmetric Balance (AB) model developed by MK97 was governed by the linearized vorticity equation (1.1) in stationary cylindrical coordinates with a simple vorticity monopole representing the mean vortex on an $f$-plane:

$$
\left(\frac{\partial}{\partial t}+\frac{\bar{v}}{r} \frac{\partial}{\partial \lambda}\right) \zeta^{\prime}-\frac{\partial \psi^{\prime}}{r \partial \lambda} \frac{d \bar{\eta}}{d r}=0
$$

The terms are as follows: time $t, \bar{v}$ is the basic-state tangential velocity at radius, $r$, azimuthal angle, $\lambda$, with $\zeta^{\prime}$ and $\psi^{\prime}$ representing the perturbation vorticity and streamfunction, respectively, and $\bar{\eta}$ is the basic-state vertical vorticity. The model solution yielded wavenumber-1 inner core and outer vorticity anomalies with different rotation periods. Outward-propagating VRW packets became nearly stationary at a 
finite, outer stagnation radius as the waves' radial wavenumbers increased through the vortex's "shearing effect." The existence of the stagnation (or critical) radius highlights the dependence of VRW propagation upon the radial vorticity gradient, which confined the waves to a narrow radial interval so that radiation to the vortex environment is not possible. It was hypothesized that forced VRWs perhaps extracted and deposited energy from one radial band to another, particularly for higher wavenumbers.

The AB approximation also distinguished between VRWs and observed, or modeled gravity-inertia waves. In fact, MK97 speculated that previous primitive equation models mistook VRWs for gravity waves. By analogy with non-divergent Rossby waves on a $\beta$-plane in a uniform zonal flow, MK97 derived a similar VRW dispersion relation (1.2), which shows that VRWs propagate against the cyclonic angular velocity in the vortex core where the mean-storm vorticity gradient, $\overline{\zeta_{0}}{ }^{\prime}$, is inherently negative:

$$
\omega=n \overline{\Omega_{0}}+\frac{n}{R} \frac{\overline{\zeta_{0}^{\prime}}}{\left(k^{2}+n^{2} / R^{2}\right)}
$$

Here, $\omega$ is the propagation frequency relative to the ground, $n \overline{\Omega_{0}}$ is the Doppler-shifted frequency, the product of the azimuthal wavenumber and basic-state angular velocity, $R$ is radius, and $k$ is the radial wavenumber. The dispersion relation can also be used to describe the propagation dynamics of stationary, trapped, or radially short and long VRWs (e.g., Chapter 3). Further notable results from AB Theory included: derived 
expressions for linear phase (1.3) and group velocities (1.4), recognition of VRWs as a vortex intensification mechanism, and the connection between spiral bands and TC evolution. Asymmetric Balance highlighted that positive (outwardly directed) VRW group velocity controlled the speed and direction of energy propagation for trailing spirals, corresponding to positive radial wavenumbers, resulting in slowing outward propagation as $n \overline{\Omega_{0}}$ decreased.

$$
\begin{gathered}
C_{p r}=\frac{n}{k} \overline{\Omega_{0}}+\frac{n}{R k} \frac{\overline{\zeta_{0}^{\prime}}}{\left(k^{2}+n^{2} / R^{2}\right)} \\
C_{g r}=\frac{-2 k n \overline{\zeta_{0}^{\prime}}}{R\left(k^{2}+n^{2} / R^{2}\right)^{2}}
\end{gathered}
$$

Lastly, AB provides important insight into VRWs' potential role in TC intensity change. The initialized stage of the TCs' life cycles yields different effects from convective forcing. If the vortex is in the formative stage, then asymmetries may provide the necessary spin up to develop further, whereas mature TCs may form secondary wind maxima that ultimately causes weakening. Exciting the waves near the radius of maximum winds (RMW) accelerated the mean tangential wind at the forcing locus, with radially broader forcing yielding a stronger response (clarified in Chapter 4), Lastly, if the outward propagation is sustained by continuous forcing, the mean tangential winds would strengthen through eddy angular momentum convergence, which was later validated in future studies. 


\section{Subsequent VRW Findings}

Inward angular momentum transport from forced VRWs caused eyewall contraction and mean-vortex intensification according to Chen and Yau (2001), Möller and Shapiro (2002), and Chen et al. (2003). The former study identified one type of resultant VRWs: prograde waves that propagate faster than tangential winds inside potential vorticity rings, have outward phase and inward group velocities, and are responsible for eddy vorticity fluxes toward the eyewall. The latter study specifically noted an acceleration of the mean tangential wind in the lower and middle troposphere inside and outside the eyewall, and a deceleration aloft in the eyewall region. There were however at least two studies (Wang 2002b and Chen and Yau 2003) that showed wavemean flow interactions actually preventing intensification because the VRWs accelerated tangential winds directly in the eye, which led to axially symmetric outflow and deceleration at the RMW. The findings discussed thus far suggest that intensity changes from VRW angular momentum transport appear to be contingent on whether the acceleration occurs in the eye or eyewall, thus begging the question, what factors govern the locus of mean-flow acceleration (answered in Chapter 4)?

In addition to angular momentum transport, asymmetric vorticity mixing from the eyewall into the eye by counter-propagating VRWs raises the possibility of an additional intensity mechanism. High-wavenumber simulations generally showed that phase-locked waves grew synchronously, leading to instability that pooled vorticity from the eyewall into the eye to produce mesovortices (Fig 11) as is sometimes observed in mature TCs (Schubert et al. 1999, Chen and Yau 2003, Kossin and Schubert 2001, Kuo et al. 1999). The inward-outward exchange of high and low vorticity between the eye and eyewall 
involves angular momentum transports as well. In addition Wang (2002b) described inward, cyclonically spiraling outer rainbands occasionally perturbing the eyewall which can cause a "breakdown," followed by a recovery through the vortex axisymmetrization process. As a result, some high vorticity from the eyewall can mix outward to produce filaments and spiral bands. The vorticity mixing mechanism can also alter the eyewall into polygonal or elliptical shapes. Such changes would surely cause RMW fluctuations which has intensity change implications.

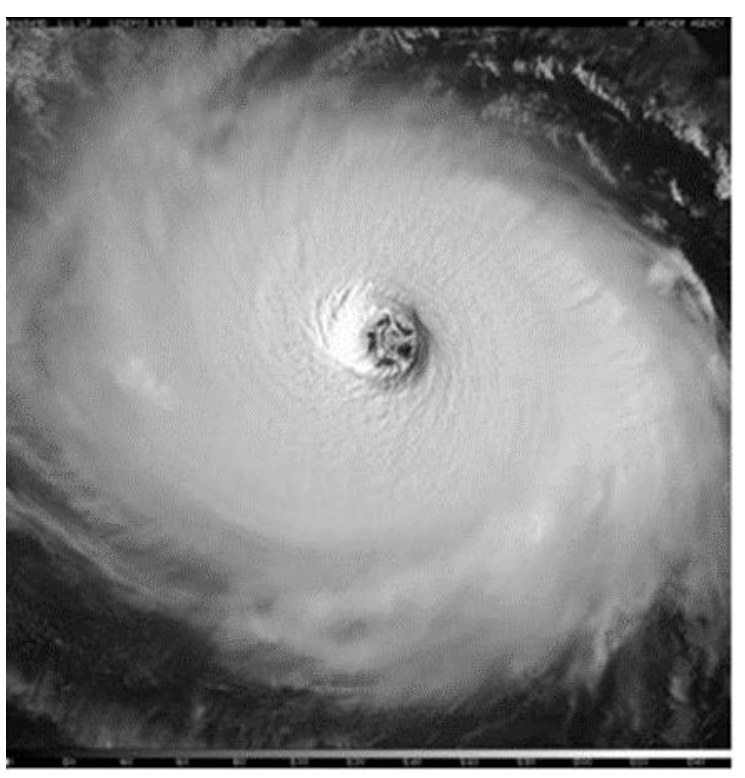

Fig 11. An example of a TC (Hurricane Isabel 2003) displaying eyewall mesovortices: Kossin and Schubert 2004.

Numerical studies summarized here describe many important aspects of VRWinduced TC dynamics, but evidence for the actual existence of the waves in real TCs remained in question, until several storms were subsequently observed. Hurricane Jimena (1991), and Hurricane Olivia (1994) were notable observational cases that generally showed low-wavenumber, cyclonically rotating eyewall perturbations, vorticity 
asymmetries, and rainbands with VRW-like properties (Muramatsu 1986, Kuo et al. 1999, Reasor et al. 2000, and Black et al. 2002). In Hurricane Olivia, spiral bands of vorticity found outside the regions of high wavenumber- 2 vorticity asymmetry appeared to be symmetrizing VRWs (Fig 12; Reasor et al. 2000). Some asymmetries exhibited rotation rates slower than the TC's angular velocity at the RMW near deep, intense convection. Hurricanes Jimena and Olivia were sheared storms with periodic convection that exhibited radar echoes and updrafts forming in the downshear quadrant. The reflectivity asymmetries were advected around the eye at $60-80 \%$ of the swirling wind, consistent with convectively excited VRW propagation (Black et al. 2002). Numerically simulating low-wavenumber VRWs forced periodically at the mean-vortex RMW by eyewall convection rotating at different specified frequencies, was therefore important to validate the observational findings.
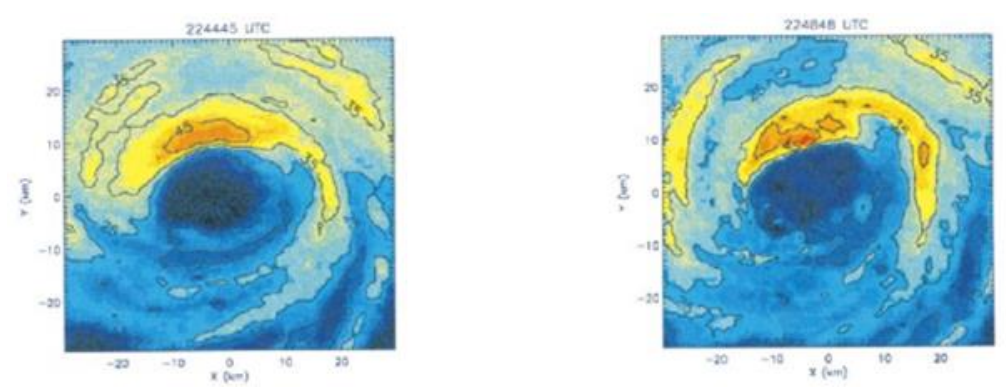

Fig 12. Radar reflectivity asymmetries associated with Hurricane Olivia (1994): Reasor et al. 2000 .

Despite better understanding of VRWs' effects on TC structure and intensity change, and wave transport, the waves' effects on TC motion, and the waveguides within which the waves propagate have received limited attention. Recently however, CGW excited barotropic, nondivergent (BND) VRWs with wavenumbers $\geq 2$ in an inner 
waveguide of a bounded vortex, with an episodic forcing, and serves as the primary motivation for this dissertation. A BND framework involves no vertical wind shear and implies that divergence, $\nabla \cdot v=0$.

Motivation

The current research primarily builds upon CGW, who first defined the inner waveguide that supports VRW propagation upon the mean vortex's negative radial vorticity gradient. Episodic forcing of wavenumber $\geq 2$ VRWs was represented as a Fourier series in stationary cylindrical coordinates. Components with specified propagation frequencies $(\omega)$ relative to the ground were superposed to synthesize the complete wavetrain. In time domain, active forcing alternated with longer quiescent intervals (Fig 13a). The forcing imposed at the mean-vortex's RMW crudely represented eyewall episodic convection rotating with $\omega$. In frequency domain, each wave component propagated radially both inward and outward away from the forcing radius.

The complete solution showed a wavetrain of alternating cyclonic and anticyclonic eddies advected downstream by the mean swirling flow (Fig 13b); although individual wave packets propagated upstream against the mean flow. Eddies appeared as $2 n$ teardrop-shaped, vorticity gyres of opposing polarity centered on the forcing, where $n$ is the tangential wavenumber. Wavenumber 2 for example, yielded two dipoles, totaling four gyres (two positive/two negative). At the critical radius, the outer edge of each gyre was distorted into trailing spirals that resembled observed TC rainbands. Nondivergent flow was described by a streamfunction whose orientation produced a balanced exchange 
of low and high vorticity between the eye and eyewall. The sunflower-like pattern was a meteorological col (Fig 14), resulting in zero net-vorticity advection across the "saddle point" at the vortex center, so that wavenumber $>1$ VRWs cannot induce vortex motion.
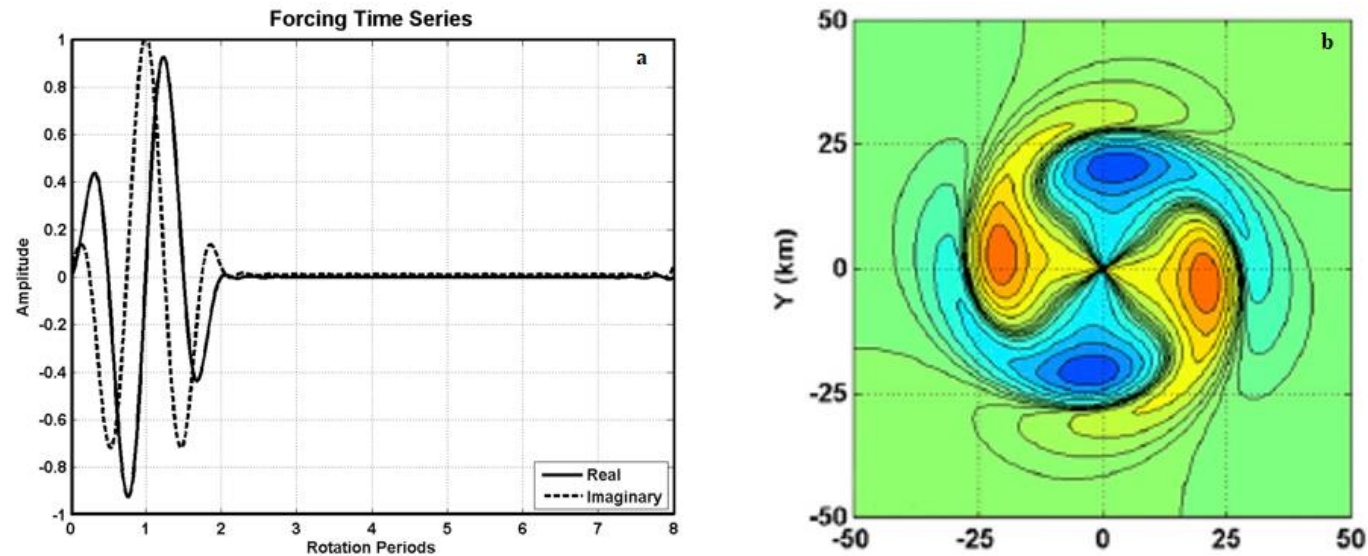

Fig 13. Wavenumber-2 forcing cycle where the oscillations represent the active interval (a) that excites VRWs that manifest as a wavetrain of tear-drop-shaped vorticity gyres advected downstream by the mean-swirling flow with outer trailing spirals (b): Cotto (2012) and Cotto et al. 2015.
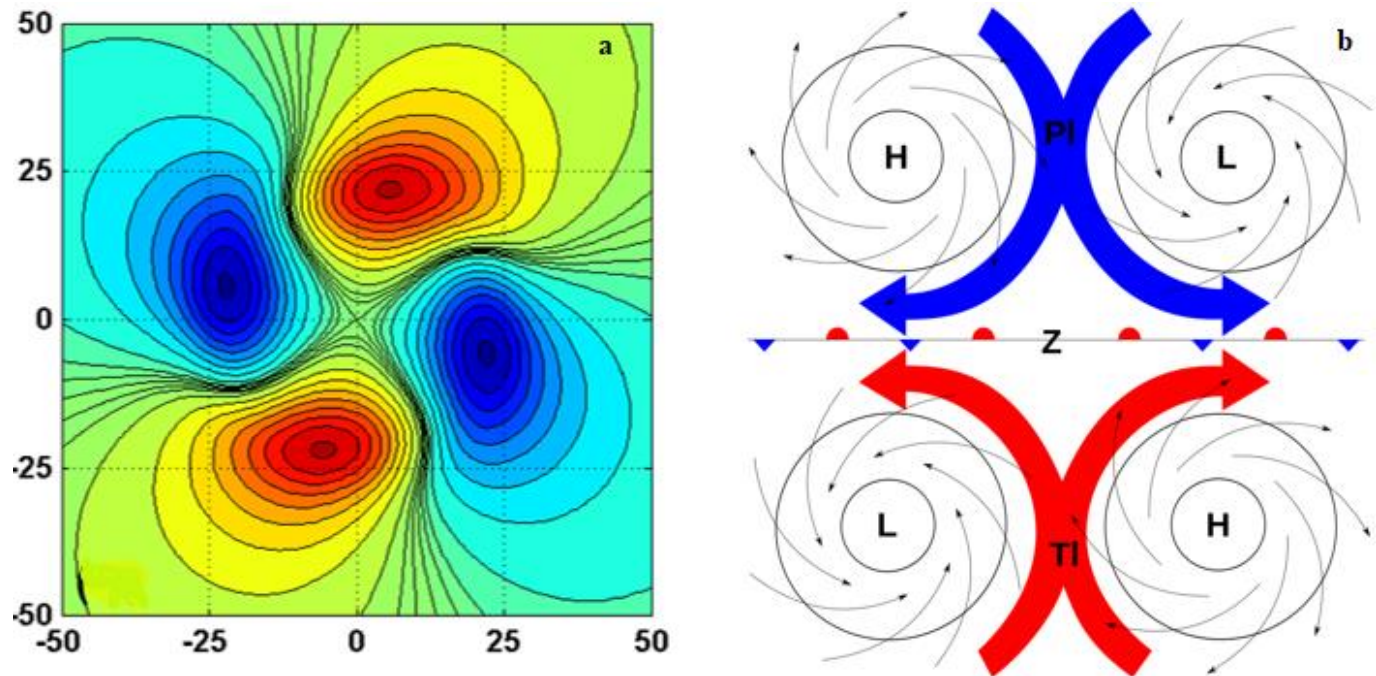

Fig 14. Wavenumber-2 streamfunction (Cotto 2012 and Cotto et al. 2015) whose sunflower-like orientation produces a saddle point in the center (a) that resembles a meteorological col (b). 
During the forcing cycle's active episodes, initially inward-propagating wave energy reflected from a turning point inside the eye, as the waves were Doppler-shifted (see dispersion relation, equation 1.2 by MK97) to the cutoff frequency at the waveguide's inner boundary. The cutoff represents the highest (most negative) frequency a VRW can have. Outward-propagating wave energy was Doppler-shifted to zero frequency at the waveguide's outer boundary to become filamented and absorbed at a critical radius. During the quiescent episodes, VRW excitation ceased but residual wave energy propagated outward to the critical radius. The significance of the inner waveguide is that intersections of a frequency passband with the cutoff and zero frequency define an annulus of free-wave propagation at the selected frequency, corresponding to a passband between zero and the cutoff Doppler-shifted frequencies (Fig 15).

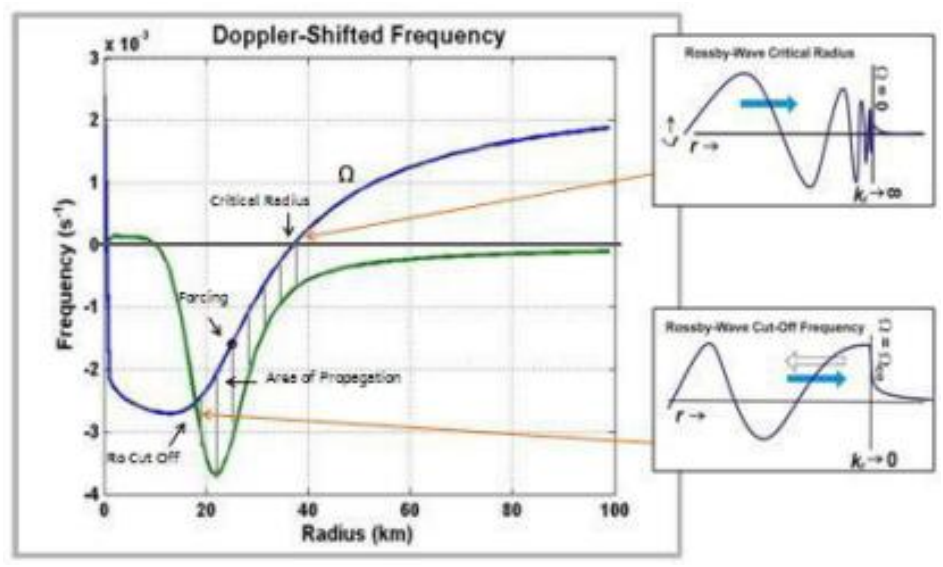

Fig 15. Forced waves at $25 \mathrm{~km}$ from the vortex center propagating with a negative Dopplershifted frequency, $\Omega$ (blue curve) within the inner waveguide bounded by the cutoff frequency, $\Omega_{1 \mathrm{D}}$ (green curve). Area of propagation and loci of energy reflection and absorption are also highlighted: Cotto 2012.

Consistent with MK97, the forced waves also converged angular momentum toward the forcing to accelerate the mean flow. The combination of initial outward radial 
propagation from the forcing radius and wave reflection from the turning point inward of the RMW produced a positive energy flux outside the forcing locus. Between the forcing radius and the turning point, the net fluxes were zero because the reflected wavetrain cancelled the initially inward-propagating wavetrain. Although CGW synthesized VRW propagation, eddy flux budgets, and waveguide frequency passbands, the model did not include wavenumber 1 because of the complexities of forced vortex motion. Furthermore, as $n$ increases, the cutoff frequency becomes less negative, thus narrowing the inner waveguide, as the critical radius moves inward (Fig 16). A narrow waveguide further confines VRWs to a smaller radial interval, reduces the distance over which wave transports act, and decreases the range of frequencies with which the waves can propagate freely. Therefore, focusing on convectively forced, wavenumber-1 VRWs is the next logical step because of the connection to motion and ability to study the widest possible inner waveguide. Clarity is also needed on the general behavior of waves at large/small radial wavenumbers (including trapped and stationary waves), and the meanflow acceleration mechanism through angular momentum convergence. 


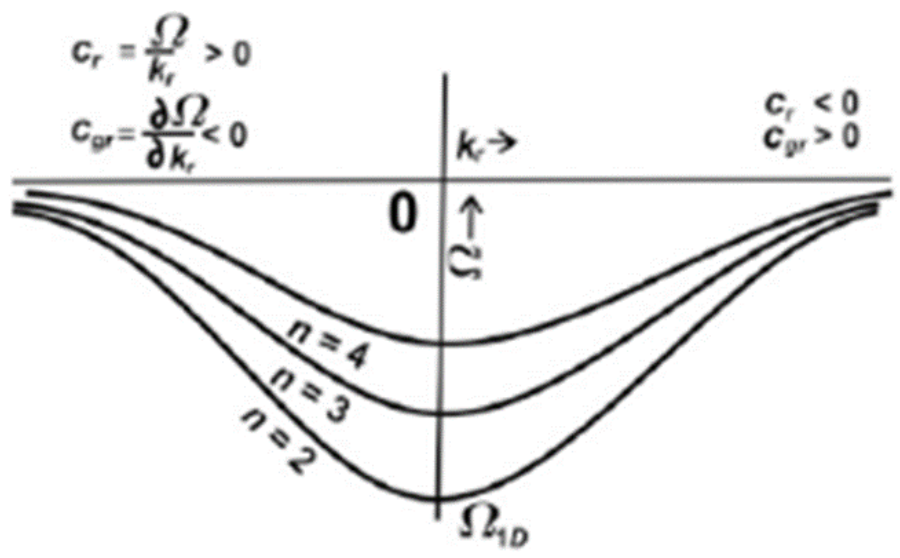

Fig 16. Vortex Rossby wave dispersion diagram illustrating how the waveguide becomes narrower with increasing tangential wavenumber, and that $\Omega$ approaches zero as radial wavenumber becomes large.

Dissertation Research Questions and Outline

The overall goal of this dissertation is to reach a different perspective on VRW dynamics in a BND context so that a more complete synthesis is attained and to apply the framework to analogous, synoptic-scale Rossby waves. Below is the outline for the remainder of the dissertation with Chapters 3-6 addressing important research questions:

- Chapter II. Formulation

- Detailed description of the model

- Equation derivations

- Describes algorithm used to obtain solutions

- Reviews VRW dispersion relation

- Chapter III. Wavenumber-1 VRWs at Different Specified Frequencies

- How does the mean-vortex structure evolve with time in response to rotational wavenumber-1 forcing imposed in the eyewall and what are the 
effects of excited VRWs propagating with different specified frequencies, on vortex motion?

- Clarification on eddy flux budgets is also obtained.

- What is the dynamical significance of narrower or wider waveguides?

- What are the properties of radially long, radially short, evanescent, and stationary waves?

What range of specified frequencies lead to radially trapped waves and what are the potential implications for vortex intensity?

○ How do wavenumber-1 results compare with wavenumber $\geq 2$ ?

- Chapter IV. Forcing Sensitivity Studies and Beta-Plane Simulations

- Can the present, barotropic nondivergent model verify Montgomery and Kallenbach (1997) findings on the following: 1) excitement of VRWs near the radius of maximum wind resulted in mean-tangential-wind acceleration at the forcing locus; 2) radially broader forcing yielded a stronger response?

What factors govern the locus of mean-flow acceleration through inward angular momentum convergence?

- How does episodic forcing affect wavenumber-1 VRW propagation and vortex motion?

- How does vortex motion change when a beta-forcing is added?

- How does model reinitialization with added beta-forcing impact motion?

- Chapter V: Vortex Sensitivity Studies and Wind-Profile Analyses 
- How does the specified intensity of the mean vortex influence VRW propagation and vortex motion?

- Are mean-vorticity monopoles consistent with dynamics on a spherical manifold?

- How do differently shaped wind profiles of bounded and unbounded vortices compare with the default, mean vortex response from Chapter 3 ?

- Chapter VI. Synoptic-Scale Rossby waves in a Meridionally Sheared, Zonal Flow

- What are the analogies between BND VRWs and synoptic-scale Rossby waves and how is the waveguide influenced by changes to specified model parameters?

- Chapter VII. Conclusions

○ Revisits research questions

- Discusses validation and future work 


\section{CHAPTER II. FORMULATION}

Model Overview and Coordinate System

An idealized BND, vortex-following model on a quiescent $f$-plane is the simplest formulation that has the physics essential to simulate 2-D convectively forced wavenumber-1 VRWs and vortex motion. The model, written in MATLAB reads the mean vortex wind profile and wave forcing properties from a setup file, then marches the vorticity equation forward in time for 24 simulated hours in 150-s time steps. After each time step, the Poisson-like vorticity equation is inverted to obtain the streamfunction. The moving vortex center is then tracked in a translating cylindrical coordinate system (Fig 17) represented by the following variables: $u$, radial velocity component (positive outward); $v$, tangential velocity component (positive cyclonically); $\lambda$, azimuth angle (reckoned cyclonically from north); $r$, radius; $c_{x}$, zonal translation (positive eastward); and $c_{y}$, meridional translation (positive northward). After vortex tracking, eddy fluxes and meanflow acceleration are calculated. The model output includes vorticity and streamfunction fields, and vortex track, speed, and direction of motion during each desired time step. 


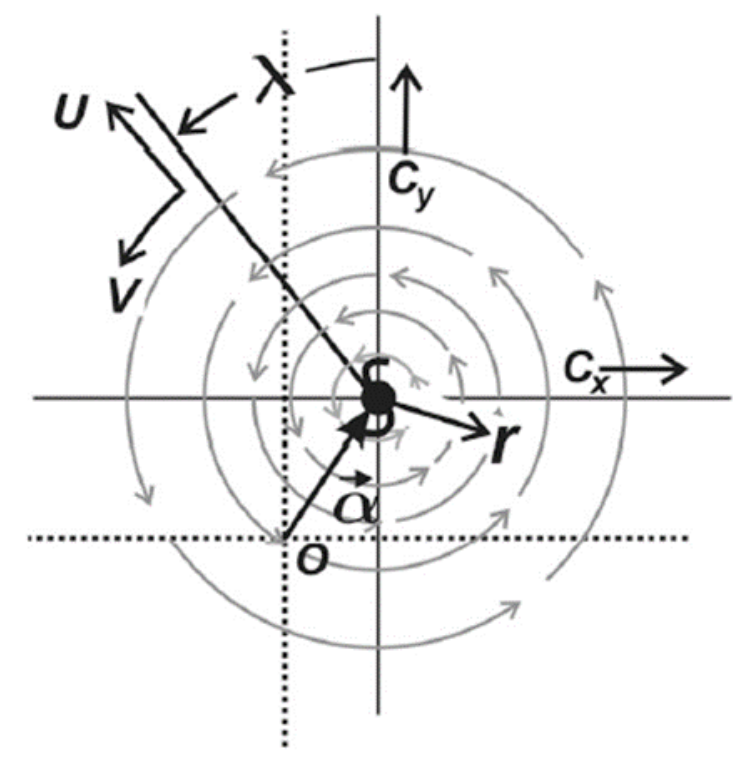

Fig 17. Mean vortex in a translating cylindrical coordinate system.

\section{Mean-Vortex Wind Profile}

The wind profile is derived from a cyclostrophic mean vortex derived from Wood and White (2010, hereafter WW), where the scaled maximum cyclostrophic wind is first calculated in order to have the maximum gradient:

$$
V_{c x}=\frac{V_{x}\left(0.5+\sqrt{0.25+\left(V_{x} / f R_{x}\right)^{2}}\right)}{V_{x} / f R_{x}}
$$

Here, $V_{x}$ is the $50 \mathrm{~ms}^{-1}$ maximum wind, and $V_{x} / f R_{x}$ is the Rossby number at the RMW, an indicator of the relative roles of the Coriolis and centripetal force; $f$ is the Coriolis 
parameter calculated at $20^{\circ} \mathrm{N}$ latitude and $R_{x}$ is the $25-\mathrm{km}$ radius of maximum wind.

Tropical cyclones exhibit large Rossby numbers $\left(10-10^{2}\right)$, implying that the cores of TCs are nearly in cyclostrophic balance. The WW cyclostrophic wind is expressed as:

$$
V_{c}(r)=V_{c x}\left\{\frac{\left(\frac{r}{R_{x}}\right)^{\frac{N_{\text {in }}}{L}}}{1+\frac{N_{\text {in }}}{N_{\text {in }}-N_{\text {out }}}\left[\left(\frac{r}{R_{x}}\right)^{\frac{N_{\text {in }}-N_{\text {out }}}{L}}-1\right]}\right\}^{L}=V_{c x}\left\{\frac{\left(\frac{r}{R_{x}}\right)^{4}}{1+\frac{2}{3}\left[\left(\frac{r}{R_{x}}\right)^{6}-1\right]}\right\}^{1 / 4}
$$

The following variables from (2) are: $r$, the radius from the vortex center; $N_{i n}$, the innervortex $\left(r<R_{x}\right)$ power-law exponent; $N_{\text {out }}$, the outer-vortex $\left(r>R_{x}\right)$ power-law exponent; and $L$, the width of the transition between the inner and outer profiles. Typical values are $N_{\text {in }}=1.25$ and $N_{\text {out }}=-0.5$. The inner and outer-vortex power-law exponents were chosen to produce solid rotation of $V_{c}$ inside the eye and inverse square root variation outside the eye with a smooth transition between regimes. In the limit of large radius and small $R_{c}, V$ becomes proportional to $r^{-2}$ (inverse $r$ from $R_{c}$ and two factors of $r^{-1 / 2}$ ) so that the circulation asymptotes to zero as $r$ becomes large. The vortex is subsequently defined as "asymptotically bounded" because the circulation approaches zero as $r$ becomes large (Gonzalez et al. 2015, hereafter GCW). Moreover, the Circulation Theorem requires an annulus of anticyclonic vorticity at the periphery, which is discussed further in Chapter 5. To produce gradient wind, $V$ at a $25-\mathrm{km}$ radius (Fig 18a), $V_{c x}$ and $R_{x}$ are adjusted as described above. A useful expression for $V$ stems from Gradient Balance where the 
pressure-gradient force ( $3^{\text {rd }}$ term from left below) is written in terms of $V_{c}$ and geostrophic wind $\left(V_{g}\right)$. The latter is a reasonable approximation to the real wind in the TC core:

$$
\frac{V^{2}}{r}+f V=\rho_{0}^{-1} \frac{\partial p}{\partial r}=f V_{g}=\frac{V_{c}^{2}}{r}
$$

Solving the quadratic equation (3) for $V$ and multiplying above and below by the conjugate root (Willoughby 2011) yields the gradient wind for anomalous flow:

$$
V=\frac{R_{c} V_{c}}{\frac{1}{2}\left[1 \pm \sqrt{1+4 R_{c}^{2}}\right]}=\frac{V_{g}}{\frac{1}{2}\left[1+\sqrt{1+4 R}_{G}\right]}
$$

Here, $R_{c}=V_{c} / f r$ and $R_{G}=V / f r$ are the cyclostrophic and gradient-wind Rossby numbers, respectively. Lastly, $V_{c x}$ is adjusted to the desired value with,

$$
V_{c x}=\frac{V_{x}}{2 R_{c x}}\left[1 \pm \sqrt{1+4 R_{c x}^{2}}\right]
$$

where $R_{c x}=V_{c x} / f R_{x} \approx V_{x} / f R_{x}$, is the scaled Rossby number which approximates the Rossby number as first defined in (1). 

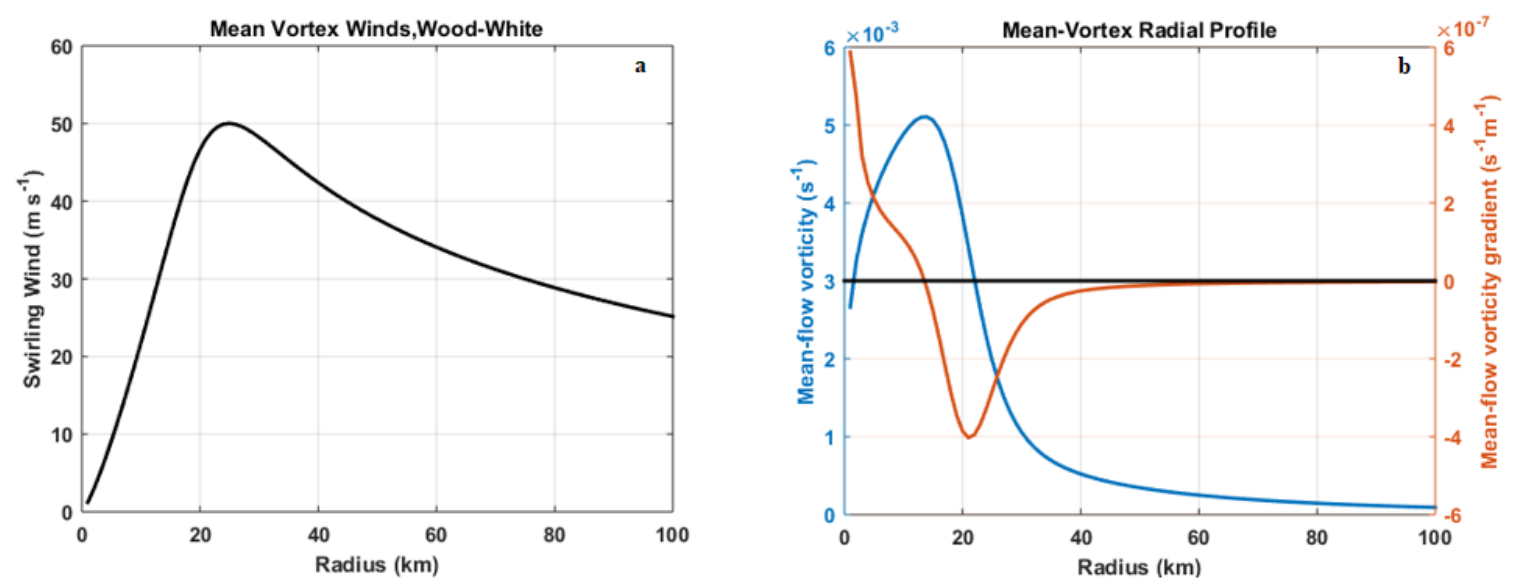

Fig 18. Mean-vortex tangential wind (a), and storm-flow vorticity (b, blue curve) and vorticity gradient profiles (b, orange curve).

\section{Vorticity Equation}

Vorticity is the essential prognostic variable in the present BND model because VRWs are vorticity waves. In cylindrical coordinates, axially symmetric mean-flow relative vorticity is expressed as $\zeta_{0}=\partial v / \partial r+v / r$, where $\partial v / \partial r$ is the tangential shear and $v / r$ is the angular velocity. Vorticity is strongly positive inside the RMW, weakens with increasing radius, and reverses sign at the periphery (Fig 18a) of the asymptotically bounded vortex. Radial vorticity gradient $(\partial \zeta / \partial r)$ is predominantly negative in the core, but is positive in a narrow radius inside the eye (Fig 18b). The vorticity equation is central to illustrating mean-vortex structural evolution and VRW propagation. The derivation begins with the governing radial and tangential momentum equations (6 and 7) in translating cylindrical coordinates (e.g., Willoughby 1992):

$$
\frac{\partial u}{\partial t}+\frac{V}{r} \frac{\partial u}{\partial \lambda}-\xi_{0}\left(v-c_{\lambda}\right)+\frac{\partial \phi}{\partial r}=F_{r}
$$




$$
\frac{\partial v}{\partial t}+\frac{V}{r} \frac{\partial v}{\partial \lambda}-\zeta_{0}\left(u-c_{r}\right)+\frac{1}{r} \frac{\partial \phi}{\partial \lambda}=F_{\lambda}
$$

On the left-hand side the terms are as follows: velocity tendency, mean-flow advection in the azimuthal direction (where $\xi_{0}=2 V / r+f$, is the inertial parameter), meanflow vorticity advection by the vortex motion, and geopotential. The cylindrical coordinate system translates with velocity, $\vec{c}=c_{r} \hat{r}+c_{\lambda} \hat{\lambda}$, where $c_{r}$ and $c_{\lambda}$ represent the radial and tangential components of the reference frame's motion, respectively. Here, $\hat{r}$ and $\hat{\lambda}$ are the corresponding unit vectors. The radial and azimuthal components of the vortex translation are expressed as, $c_{r}=c_{y} \cos \lambda-c_{x} \sin \lambda$ and $c_{\lambda}=-c_{x} \cos \lambda-c_{y} \sin \lambda$, respectively (refer to Fig 17). Complex motion is defined as $C=c_{x}+i c_{y}$. Applying Euler's Formula gives:

$$
\begin{gathered}
c_{r}=\operatorname{Re}\left\{-i\left(c_{x}+i c_{y}\right) e^{-i \lambda}\right\}=\operatorname{Re}\left\{-i C e^{-i \lambda}\right\} \\
c_{\lambda}=\operatorname{Re}\left\{\left(c_{x}+i c_{y}\right) e^{-i \lambda}\right\}=\operatorname{Re}\left\{C e^{-i \lambda}\right\}
\end{gathered}
$$

On the right-hand side of (6) and (7) are the imposed momentum forces derived from a vector forcing potential, $A$, such that $F_{r}=-r^{-1}(\partial A / \partial \lambda)$ and $F_{\lambda}=\partial A / \partial r$ act only on the rotational part of the wind, as described by CGW. Analogous to $u$ and $v$ in translating cylindrical coordinates, $F_{r}$ and $F_{\lambda}$ are positive outward and cyclonically, respectively. Taking $r^{-1} \partial / \partial \lambda$ of (6) and $\partial / \partial r+1 / r$ of (7), followed by subtraction eliminates the geopotential to yield the nondivergent vorticity equation: 


$$
\left(\frac{\partial}{\partial t}+\frac{V}{r} \frac{\partial}{\partial \lambda}\right)\left(\frac{\partial v}{\partial r}+\frac{v}{r}-\frac{1}{r} \frac{\partial u}{\partial \lambda}\right)+\left(u-c_{r}\right) \frac{\partial \zeta}{\partial r}=\frac{\partial^{2} A}{\partial r^{2}}+\frac{1}{r} \frac{\partial A}{\partial r}+\frac{1}{r^{2}} \frac{\partial^{2} A}{\partial \lambda^{2}} \equiv Q
$$

The first term in parenthesis is the linearized Lagrangian derivative of $\zeta$ in expanded form. The second term is advection of mean-flow relative vorticity by the perturbation flow and motion of the vortex. On the right-hand side is the imposed forcing, $Q$, expressed as the Laplacian of $A$. Rearranging the terms yields the vorticity tendency equation (11) which is expanded using finite-differences to march $\zeta$ forward in time:

$$
\frac{\partial \zeta}{\partial t}=-\frac{V}{r} \frac{\partial \zeta}{\partial \lambda}-\left(u-c_{r}\right) \frac{\partial \zeta}{\partial r}+Q
$$

Vorticity tendency is a first-order derivative expressed as, $\partial \zeta / \partial t=\left[\zeta_{k+1}-\zeta_{k-1}\right] / 2 \delta t$, in finite differences, where $k$ represents an index radial grid point of the domain, and $\delta t$ is the 150-s time step.

Forcing

Wavenumber-1 VRWs are excited by the steadily, rotating forcing imposed at the RMW. A polynomial bell function centered at $25-\mathrm{km}$ with $10-\mathrm{km}$ width defines the radial structure of the forcing. The forcing varies sinusoidally in azimuth and rotates with constant $\omega$ and amplitude. Unlike CGW however, the forcing is non-episodic so that the asymmetries' appearance remains unchanged throughout the simulation (apart from rotation). The dipole rotates with time, at frequency $\omega$ (Fig 19a), which is specified 
as a fraction of the mean-flow angular velocity (Fig 19b) at the RMW: $\omega_{\mathrm{o}}=V_{c x} / R_{x}=$ $2 \times 10^{-3} \mathrm{~s}^{-1}$. A range of four different specified frequencies is used to evaluate the effects of different rotation rates: $\omega=0.25 \omega_{\mathrm{o}}, 0.50 \omega_{\mathrm{o}}, 0.75 \omega_{\mathrm{o}}$, and $\omega_{\mathrm{o}}$ corresponding to orbital periods $(T=2 \pi / \omega)$ between approximately 3.5 and $0.87 \mathrm{hrs}$ (i.e., 7 to 28 revolutions per simulation). The forcing simulates TC eyewall convection as a cyclonically rotating mass source-sink pair. The mass sink produces convergence leading to vorticity stretching, whereas the mass source destroys vorticity. To a first approximation, vortex motion should consequently be toward the sink to accommodate the increased vorticity, and away from the source (Willoughby 1988).
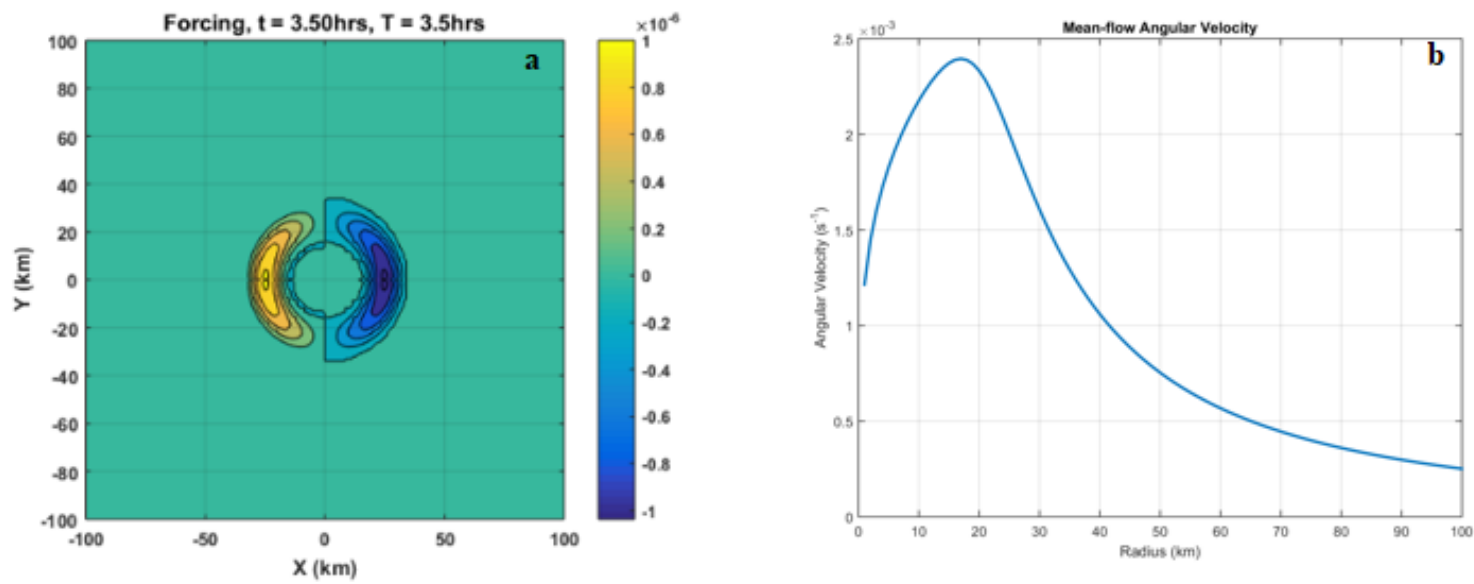

Fig 19. Forcing contour plot (a) rotating at $25 \%$ of the mean-flow angular velocity (b) at the RMW; 3.5-hr orbital period.

Streamfunction and Geopotential Solutions

After calculating $\zeta$, a Poisson inversion is used to solve for streamfunction, $\psi(r, \lambda, t)$, to represent the nondivergent flow such that, $u=-r^{-1} \partial \psi / \partial \lambda$ and $v=\partial \psi / \partial r$. The 
streamfunction acts as an effective smoother that can highlight important radial processes that $\zeta$ may mask. Equation (10) subsequently transforms into a second-order, partial differential equation:

$$
\left(\frac{\partial}{\partial t}+\frac{V}{r} \frac{\partial}{\partial \lambda}\right)\left(\frac{\partial^{2} \psi}{\partial r^{2}}+\frac{1}{r} \frac{\partial \psi}{\partial r}+\frac{1}{r^{2}} \frac{\partial^{2} \psi}{\partial \lambda^{2}}\right)-\left(\frac{1}{r} \frac{\partial \psi}{\partial \lambda}+c_{r}\right) \frac{\partial \zeta}{\partial r}=Q
$$

The wavenumber-1 VRW solution at frequency, $\omega$ is the real part of the product of an imaginary exponential in azimuth with the complex amplitude of $\Psi$ such that, $\psi=$ $\operatorname{Re}\left\{\Psi(r, t) e^{i(\omega t-\lambda)}\right\}:$

$$
i \Omega\left(\frac{d^{2} \Psi}{d r^{2}}+\frac{1}{r} \frac{d \Psi}{d r}-\frac{\Psi}{r^{2}}\right)-\left(\frac{i \Psi}{r}-i C\right) \frac{\partial \zeta}{\partial r}=Q
$$

Here, $\Omega=\omega-V / r$, is the Doppler-shifted frequency - the sense of VRW propagation away from the source of excitation. Geopotential is another important variable that is used for calculating energy fluxes carried by propagating VRWs. Taking $\partial / \partial r+1 / r$ of (6) and $-i / r$ of (7), followed by subtraction produces a Poisson-like equation for $\phi$, where the nondivergent flow is again represented by $\psi$ :

$$
\left(\frac{\partial^{2} \phi}{\partial r^{2}}+\frac{1}{r} \frac{\partial \phi}{\partial r}+\frac{1}{r^{2}} \frac{\partial^{2} \phi}{\partial \lambda^{2}}\right)=-\frac{2}{r}\left(\zeta_{0}-\xi_{0}\right)\left(-\frac{1}{r} \frac{\partial^{2} \psi}{\partial \lambda^{2}}-\frac{\partial \psi}{\partial r}\right)+\xi_{0}\left(\frac{\partial^{2} \psi}{\partial r^{2}}+\frac{1}{r} \frac{\partial \psi}{\partial r}+\frac{1}{r^{2}} \frac{\partial^{2} \psi}{\partial \lambda^{2}}\right)
$$


Since the nondivergent flow is rotational and the forcing is derived from a vector potential (Cotto 2012), the divergence of wind and forcing that would be in (14) is zero. As with $\psi$, the assumed VRW solution for wavenumber-1 geopotential is: $\phi=$ $\operatorname{Re}\left\{\Phi(r, t) e^{i(\omega t-\lambda)}\right\}$, which transforms (14) to,

$$
\left(\frac{d^{2} \Phi}{d r^{2}}+\frac{1}{r} \frac{d \Phi}{d r}-\frac{\Phi}{r^{2}}\right)=-\frac{2}{r}\left(\zeta_{0}-\xi_{0}\right)\left(\frac{\Psi}{r}-\frac{d \Psi}{d r}\right)+\xi_{0}\left(\frac{d^{2} \Psi}{d r^{2}}+\frac{1}{r} \frac{d \Psi}{d r}-\frac{\Psi}{r^{2}}\right)
$$

Equations (13) and (15) are second-order partial differential equations that are readily solved for $\Psi$ or $\Phi$, using the Lindzen and Kuo (1969) algorithm. For convenience, the algorithm is shown for only $\Psi$. The $\Psi$-derivatives from (13) are first converted into finite differences, where the second-order derivative, $\partial^{2} \Psi / \partial r^{2}$ is expressed as, $\left[\Psi_{k+1}-2 \Psi_{k}+\Psi_{k}\right.$ $\left.{ }_{1}\right] /(\delta r)^{2} ; k$ is the index and $\delta r$ is the radial grid size. Then combining like terms yields:

$$
\left[\frac{1}{(\delta r)^{2}}+\frac{1}{r 2 \delta r}\right] \Psi_{k+1}+\left[-\frac{2}{(\delta r)^{2}}-\frac{1}{r^{2}}+\frac{1}{\Omega r} \frac{\partial \zeta}{\partial r}\right] \Psi_{k}+\left[\frac{1}{(\delta r)^{2}}-\frac{1}{r 2 \delta r}\right] \Psi_{k-1}=\Xi_{k}
$$

Each term in square brackets is defined as a "Lindzen-Kuo coefficient" such that (16) is simplified to: $A_{k} \Psi_{k+1}+B_{k} \Psi_{k}+C_{k} \Psi_{k-1}=\Xi_{k}$, where $\Xi_{k}$ is the imposed forcing. The algorithm is essentially a tri-diagonal matrix solver. The elements of the lower-diagonal intermediate step are represented by (17), where $\alpha_{k}$ and $\beta_{k}$ are two newly introduced coefficients (18 and 19): 


$$
\begin{gathered}
\Psi_{k}=\alpha_{k} \Psi_{k+1}+\beta_{k} ; \Psi_{k-1}=\alpha_{k-1} \Psi_{k}+\beta_{k-1} \\
\alpha_{k}=\frac{-A_{k}}{C_{k} \alpha_{k-1}+B_{k}} \\
\beta_{k}=\frac{\Xi_{k}-C_{k} \beta_{k-1}}{C_{k} \alpha_{k-1}+B_{k}}
\end{gathered}
$$

Substituting (18) and (19) into (17), for $k=2,3, \ldots K, K-1$, obtains the solution, where $K$ is some arbitrary index value. As with $\mathrm{CGW}$, the algorithm requires boundary conditions at the vortex center and at the outer boundary of the domain. Since the inner and outer boundaries lie outside the inner waveguide, $\Psi_{1}=0$ and $\Psi_{K}=0$, so that $\alpha_{1}=\beta_{1}=0$. The arrays of $\alpha_{1}, \alpha_{2}, \ldots, \alpha_{K-1}$ and $\beta_{1}, \beta_{2}, \ldots \beta_{K}$ are computed successively using (18) and (19) in an outward pass from $k=2, \ldots K-1$. Back substitution then becomes an inward pass across the domain to compute $\Psi_{K-1}, \Psi_{K-2, \ldots} \Psi_{1}$. The same procedure is applied to solve for $\Phi$ from (15).

Eddy Fluxes and Mean-Flow Acceleration

The forced VRWs also produce angular momentum and geopotential fluxes within the waveguide that affect the distribution of the mean flow and energy transport. To calculate eddy fluxes, the motion components from (8) and (9) must first be subtracted from the corresponding relative velocities to obtain the absolute velocities: $u_{A}$ $=u-c_{r}$ and $v_{A}=v-c_{\lambda}$. Interactions between the real parts of the exponential solutions for $u_{A}, v_{A}$, and $\phi$ involve products of complex conjugates (denoted by an asterisk) that 
yield the angular momentum and geopotential flux; the final terms on the right-hand side of (20) and (21).

$$
\begin{aligned}
& \operatorname{Re}\left\{u_{A} e^{-i \lambda}\right\} \operatorname{Re}\left\{v_{A} e^{-i \lambda}\right\}=\frac{1}{2}\left[\left(u_{A} e^{-i \lambda}+u_{A}^{*} e^{i \lambda}\right)\left(v_{A} e^{-i \lambda}+v_{A}^{*} e^{i \lambda}\right)\right]=\frac{1}{2}\left[\operatorname{Re}\left\{u_{A} v_{A} e^{-2 i \lambda}\right\}+\operatorname{Re}\left\{u_{A} v_{A}^{*}\right\}\right] \\
& \operatorname{Re}\left\{u_{A} e^{-i \lambda}\right\} \operatorname{Re}\left\{\phi e^{-i \lambda}\right\}=\frac{1}{2}\left[\left(u_{A} e^{-i \lambda}+\phi_{A}^{*} e^{i \lambda}\right)\left(\phi e^{-i \lambda}+\phi_{A}^{*} e^{i \lambda}\right)\right]=\frac{1}{2}\left[\operatorname{Re}\left\{u_{A} \phi e^{-2 i \lambda}\right\}+\operatorname{Re}\left\{u_{A} \phi^{*}\right\}\right]
\end{aligned}
$$

Some of the interactions go into the forcing of the symmetric flow of the vortex whereas energy interaction occurs at the critical radius. Therefore it is expected that angular momentum flux, $\langle u v\rangle$ converges inward towards the forcing and geopotential flux, $\langle u \phi\rangle$ convergence is towards the critical radius. The former accelerates the mean flow $(\partial v / \partial t)$. Since BND frameworks obey mass continuity such that $\partial u / \partial r+u / r=r^{-1} \partial(u r) / \partial r=0$, mean-flow acceleration should be entirely from eddy convergence at the locus of forcing and deceleration at the critical radius. Eddy flux budgets and mean-flow acceleration are presented in Chapter 3.

Wavenumber-1 Nondivergent VRW Dispersion Relation

To gain insight into VRW propagation within the inner waveguide, the dispersion relation (consistent with equation 1.2) is re-examined. If $\Psi$ is represented with zero-order Hankel functions, (13) becomes, 


$$
\Omega=\omega-\frac{V}{r}=\frac{\frac{1}{r} \frac{\partial \zeta}{\partial r}}{k_{r}^{2}+\frac{1}{r^{2}}}
$$

where $V / r$ is the mean-flow orbital frequency, and $k_{r}$ is the local radial wavenumber. Long VRWs (small $k_{r}$ ) approach the frequency of a one-dimensional VRW, effectively the cutoff frequency: $\Omega \rightarrow \Omega_{1 \mathrm{D}}=r(\partial \zeta / \partial r)$, that defines the inner boundary of the waveguide. Short waves (large $k_{r}$ ) have Doppler-shifted frequencies that approach zero, $\Omega \rightarrow 0$. Radial phase (23) and group velocities (24) are obtained as in MK97 from dividing (22) by $k_{r}$ and differentiating with respect to $k_{r}$.

$$
\begin{gathered}
C_{p}=\frac{\omega}{k_{r}}=\frac{1}{k_{r}}\left[\frac{V}{r}+\frac{\frac{1}{r} \frac{\partial \zeta}{\partial r}}{k_{r}^{2}+\frac{1}{r^{2}}}\right] \\
C_{g}=\frac{\partial \omega}{\partial k_{r}}=\frac{-2 k_{r} \frac{\partial \zeta}{\partial r}}{r\left(k_{r}^{2}+\frac{1}{r^{2}}\right)^{2}}
\end{gathered}
$$

Since the VRWs' phase and group velocities are functions of $k_{r}$, the waves are dispersive. Within the inner waveguide $\partial \zeta / \partial r<0$; therefore, the waves' phase propagation is upstream $\left(C_{p}<0\right)$ against the mean swirling flow while the wavetrain is generally advected downstream. Choosing $k_{r}$ determines the sense of radial propagation $-k_{r}$ can be positive (outward phase, inward group velocity) or negative (inward phase, outward group velocity). Frequency passbands imposed in the inner waveguide are investigated 
further in Chapter 3 to define free-wave propagation and explain what happens to the wave energy and angular momentum fluxes at the waveguide's boundaries. 


\title{
CHAPTER III. WAVENUMBER-1 VRWS AT DIFFERENT SPECIFIED
}

\section{FREQUENCIES}

\author{
General Overview
}

Chapter 3 presents key results for the following range of specified frequencies: $\omega$ $=0.25 \omega_{\mathrm{o}}, 0.50 \omega_{\mathrm{o}}, 0.75 \omega_{\mathrm{o}}$, and $\omega_{\mathrm{o}}$. The forced wavenumber-1 dipole's appearance and orientation are first described, with $\zeta$ highlighting vortex structural evolution and VRW activity, and $\psi$ illustrating the nondivergent flow. Next, vortex motion is examined by describing the track, speed, and direction. Then, wave transport is explained in terms of angular momentum and geopotential fluxes. The former determines mean-flow acceleration. Frequency passbands are then examined carefully, which helps to understand how wavenumber-1 VRWs propagate within the inner waveguide. In addition, radially trapped waves are considered to explore the potential implications for vortex intensity. Lastly, wavenumber-1 results are compared with higher-wavenumber VRWs.

Results

\section{Mean-Vortex Structural Evolution}

At the beginning of the simulation, the $10-\mathrm{km}$ wide forcing turns on and produces a wavenumber-1 dipole (Fig 21b), with an initial east-west orientation. The two gyres rotate around the center with frequency $\omega$ and peak amplitude at the 25-km RMW. 
Warm (cool) colors correspond to positive (negative) forcing, and green colors represent zero forcing. Since the forcing is continuous, only the gyres' orientation changes with time as the dipole rotates. For example, $\omega=0.25 \omega_{0}$ corresponds to a 3.5 -hr orbital period (T), or approximately 7 full rotations per simulated day. The dipole's behavior is comparable to an algebraic instability solution associated with wavenumber-1 disturbances in TC-like vortices where a growing discrete mode's structure does not change in time and rotates at a constant angular velocity (Nolan and Montgomery 1999, hereafter NM99).

Vorticity is a rotating wavenumber- 1 dipole at the forcing radius, $R_{Q}$. A small dipole is also apparent near the center of the vortex. The near-center dipole forms from the perturbation velocities interacting with the "slug" of positive radial vorticity gradient inside of the RMW (refer to Fig 18b), as described by NM99. The perturbations symmetrize with time (Fig 20a-c) as $\zeta$ becomes highly filamented at the critical radius to produce an outer ring of tightly wound, interlocking spirals resembling observed outer rainbands. As an observational comparison, the inner eyewall and outer band to the west of Hurricane Matthew on radar imagery (Fig 20d) resembles the forced dipole and the symmetrizing ring of $\zeta$ at the critical radius, and rainbands representing VRWs at different frequencies. Ripple-like filaments seen between the dipole and ring indicate outward VRW propagation from $R_{Q}$. Filaments are consistent with the algebraic instability solution where "residuals" decay with time - described by NM99 as a collection of sheared VRWs that are trapped in the vortex core. Outside of the ring is the evanescent region where some wave energy leaks past the critical radius and decays exponentially to produce a low-amplitude "wake" ahead and behind the moving vortex. 
Interestingly, the wake also aligns with the vortex's direction of motion at any given time, which may be attributed to anticyclonic (or less cyclonic) vorticity from the outer vortex environment, brought in by the slipstream ahead of the storm. Slipstream flow (Fig 21b) always exactly opposes the vortex's translation through the quiescent environment and diverts around the core. Lastly, as $\omega$ increases ( $T$ decreases), the $\zeta$ ring shrinks, because of the higher angular velocity relative to the ground (Fig 22).
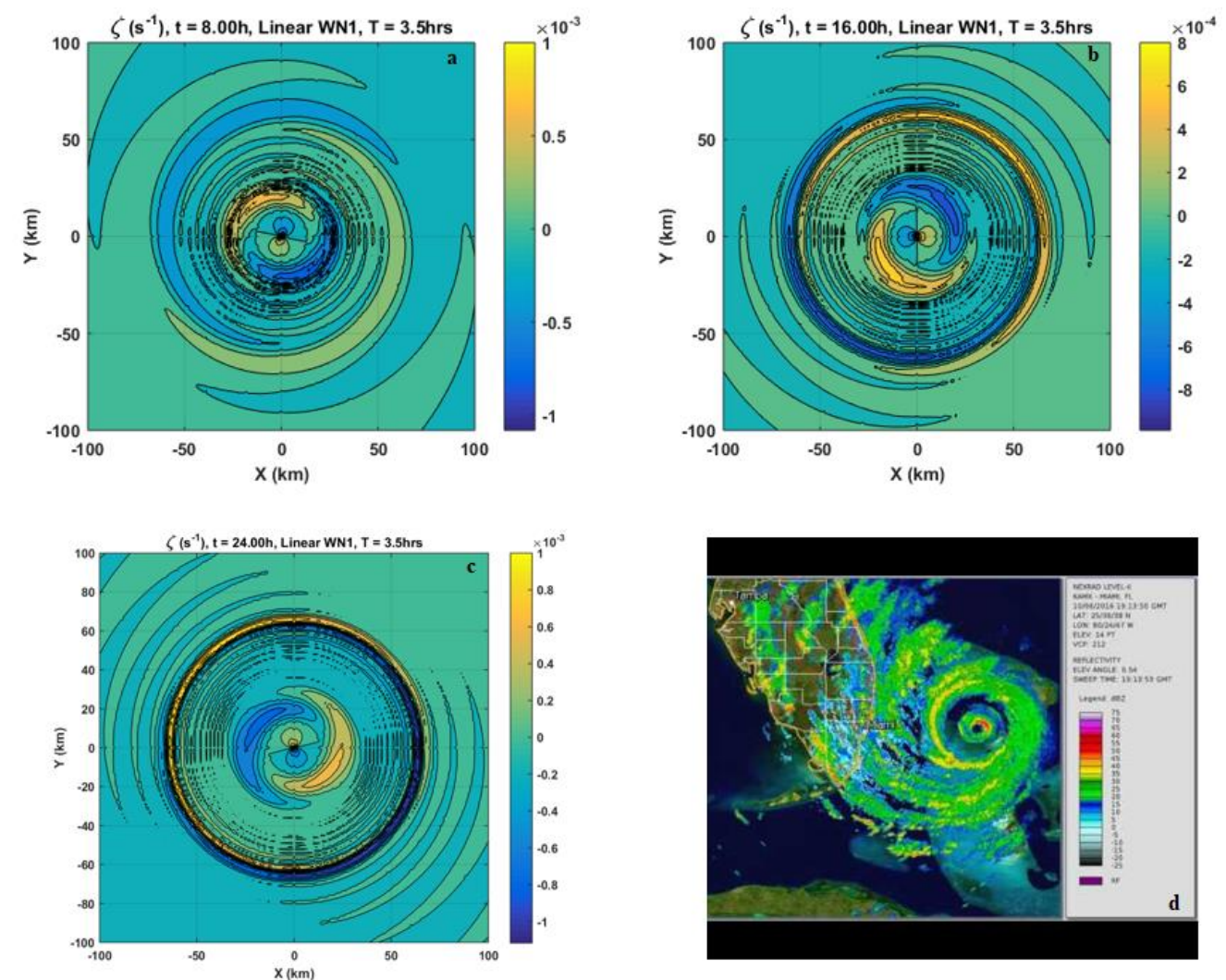

Fig 20. Vorticity contour plots at 8 -hr intervals $(\mathrm{a}, \mathrm{b}, \mathrm{c})$ highlighting mean-vortex structural evolution and a comparative radar image of Hurricane Matthew (2016) showing an outer concentric eyewall (d). 

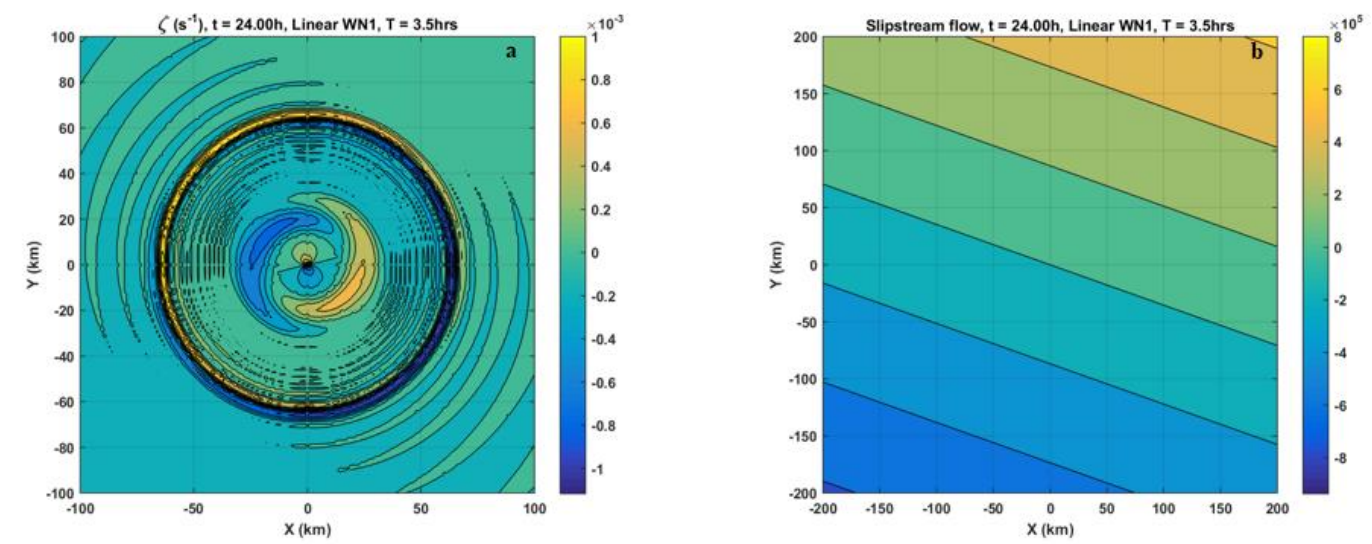

Fig 21. Vorticity contour plots (a) for a 3.5-hr forcing orbital period showing the outer wake aligning with the southeasterly slipstream flow (b).
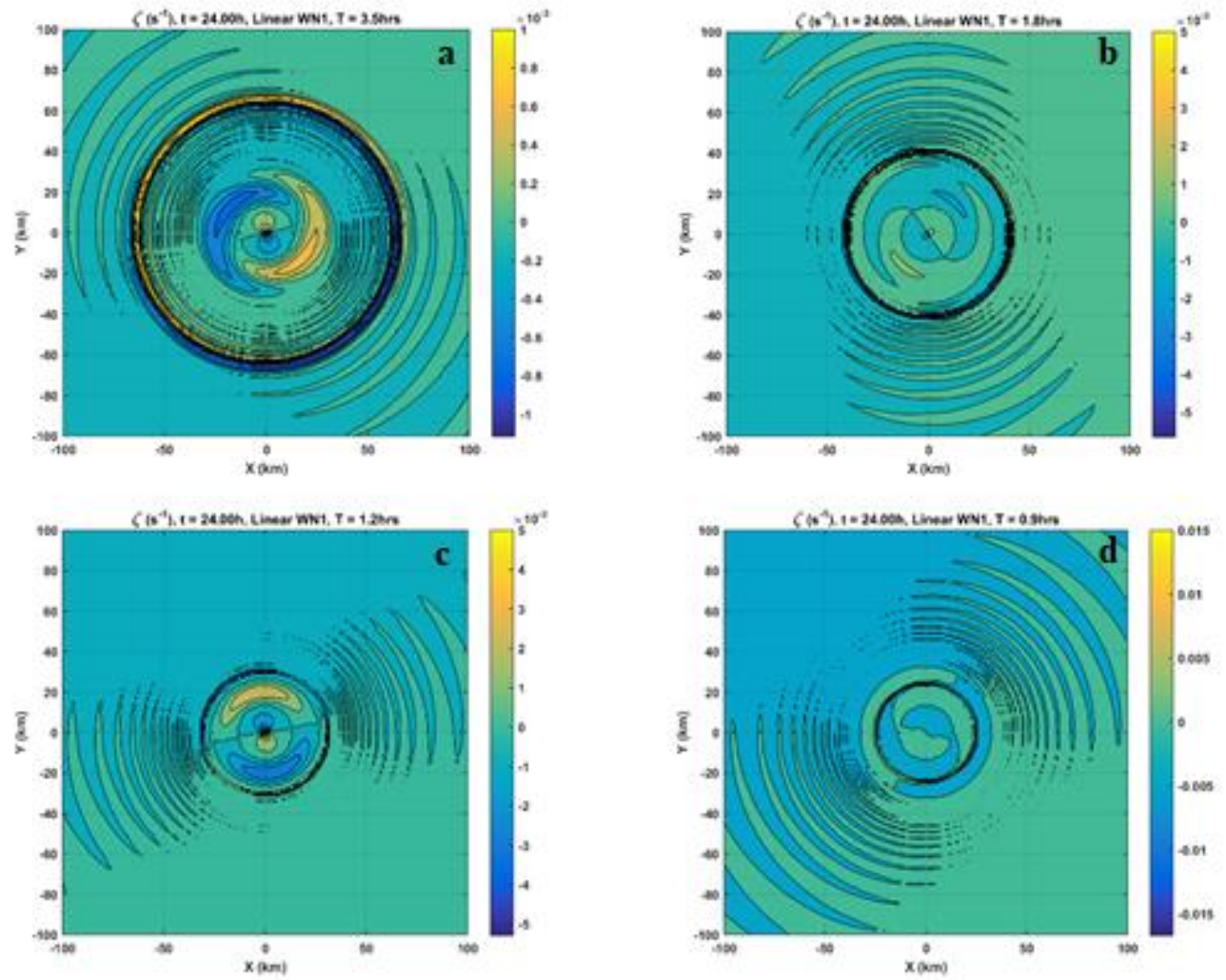

Fig 22. Vorticity contour plots for different specified orbital periods: 3.5 hrs (a), $1.8 \mathrm{hrs}$

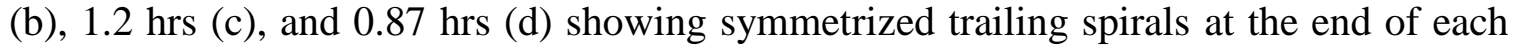
simulation. 
For most frequencies, $\zeta$ radial structure consists of two important signals: 1) near the RMW where the forcing is imposed and the dipole arises; 2) filaments in the neighborhood of the critical radius (Fig 23). The high-frequency/low-amplitude, oscillation between the two signals, corresponds to the ripple-like filaments described earlier. Beyond the critical radius, the oscillations rapidly decay to zero, from evanescent wave decay. The large differences in amplitude inward and outward from the critical radius results in a strong vorticity gradient $(\partial \zeta / \partial r)$. Faster specified frequencies compress the wave pattern which causes $\partial \zeta / \partial r$ to migrate inward. Ultimately for $\omega=\omega_{0}$, the critical radius overlaps the forcing region so that the propagating waves connect with the forcing largely through radially evanescent perturbations, consistent with CGW. Though $\zeta$ is important, $\psi$ offers a different perspective on understanding the forced dipole structure and flow field. 

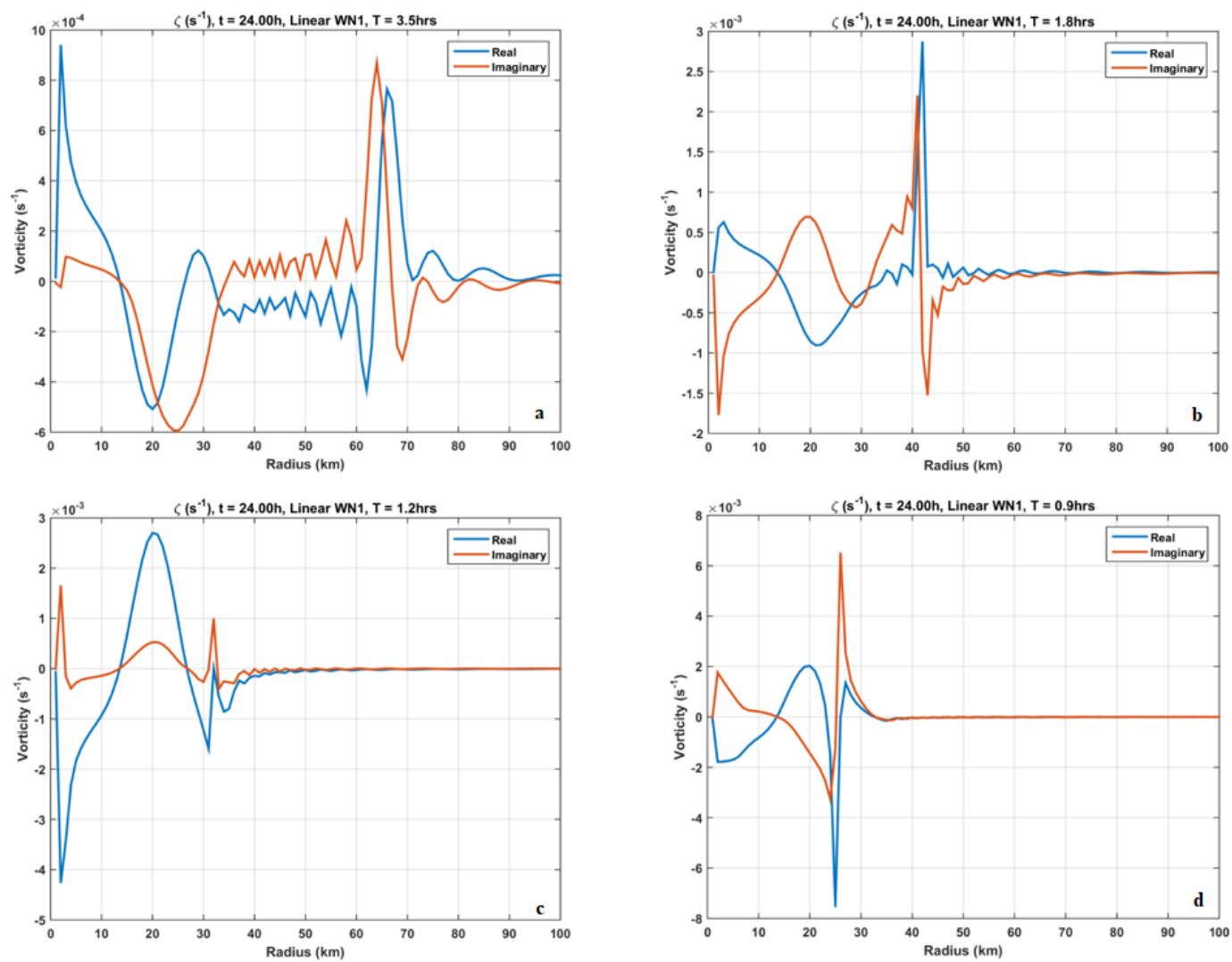

Fig 23. Vorticity radial structure for different specified orbital periods: $3.5 \mathrm{hrs}$ (a), $1.8 \mathrm{hrs}$ (b), $1.2 \mathrm{hrs}$ (c), and $0.87 \mathrm{hrs}$ (d), with anomalies highlighting the locus of forcing, propagating VRWs, and critical radius.

Streamfunction (Fig 24) is obtained by inverting the Poisson equation forced by vorticity, so warm (cool) colored gyres represent anticyclonic (cyclonic) flow. Inversion is an effective smoother that reveals important features that vorticity masks. An inner wavenumber-1 dipole forced by the rotating mass source-sink pair, combines with a secondary, larger outer dipole. An explanation for the outer dipole was offered by NM99 where $\partial \zeta / \partial r$ increases between the outward-spiraling, low-vorticity region of the eye and the high vorticity in the eyewall, leading to the appearance of secondary instabilities. Wavenumber-1 $\psi$ produces a two-fold flow field: 1) cross-vortex vorticity advection between the inner gyres; 2) inflow and outflow on opposite ends of the vortex core in 
directions that align with the vortex motion and the slipstream flow. The $\psi$ dipole orientation is different than CGW (refer to Fig 14a) because high-wavenumber solutions are not associated with vortex motion. Therefore, the present $\psi$ configuration does not result in a balanced exchange of vorticity between the eye and eyewall between counterrotating gyres. Instead, the vortex center moves in response to the gyres' rotation.
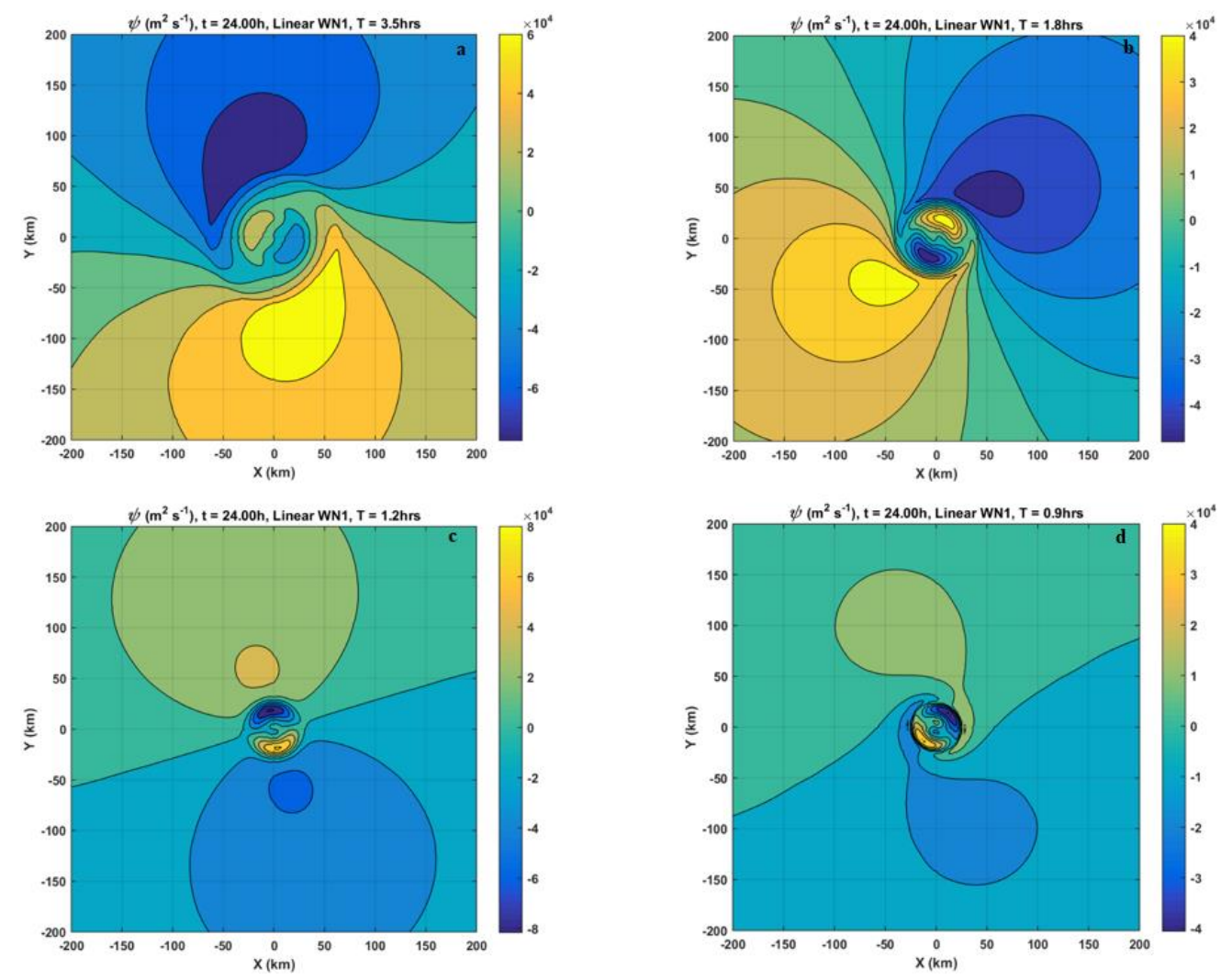

Fig 24. Streamfunction contour plots for different specified orbital periods: 3.5 hrs (a), 1.8 hrs (b), 1.2 hrs (c), and $0.87 \mathrm{hrs}$ (d) highlighting the nondivergent flow.

\section{Vortex Motion}

The quiescent environment has zero large-scale steering flow on an $f$-plane.

Therefore, only imposed vorticity sources and sinks can force the vortex to move. For 
any cyclonic $\omega$ the vortex center follows a track that initially spirals cyclonically outward from the initial position (Fig 25). Assuming nearly circular orbits, this phenomenon is likened to the steadily growing wobble of the low-vorticity region near the eye, which is associated with a net inward transport of high vorticity, as noted by NM99 and often seen in real TCs. Start-up transient growth from an abrupt turning on of the forcing is responsible for the initially chaotic motion before the solution stabilizes for the remainder of the simulation (Fig 26a). The motion is characterized by orbital speed and directionof-motion oscillations with period, $T$. For example, $T=3.5 \mathrm{hrs}$ results in the vortex center completing $\sim 7$ orbits in $24 \mathrm{hrs}$ (Fig 26b).

Mean orbital speeds and radii vary only slightly between $2.42 \mathrm{~ms}^{-1}$ to $3.65 \mathrm{~ms}^{-1}$ and $\sim 1-5 \mathrm{~km}$, respectively. Both motion parameters are sensitive to $\omega$. A physical explanation is that the presence of VRWs accumulating at the critical radius produces a high-vorticity anomaly, thus creating a vorticity deficit between the vortex center and critical radius. To compensate, the low-vorticity center becomes displaced to "narrow the vorticity gap" between itself and the critical radius. Therefore, the larger the critical radius, the greater the displacement. Orbital radius is the ratio of $\omega$ to orbital speed, so that faster forcing rotations require larger orbits. The speed generally increases incrementally with decreasing $T$, except when $\omega=\omega_{0}$, where the vortex had the slowest speed. By contrast, W92 showed that orbital period matched the period of motion and the amplitude reached a maximum value when the forcing rotated with mean-vortex flow's orbital period at a radial grid point where forcing was strongest. Additionally, the growing displacement of the low-vorticity center in the present model, results in angular momentum redistribution (as in NM99), discussed in the next subsection. 

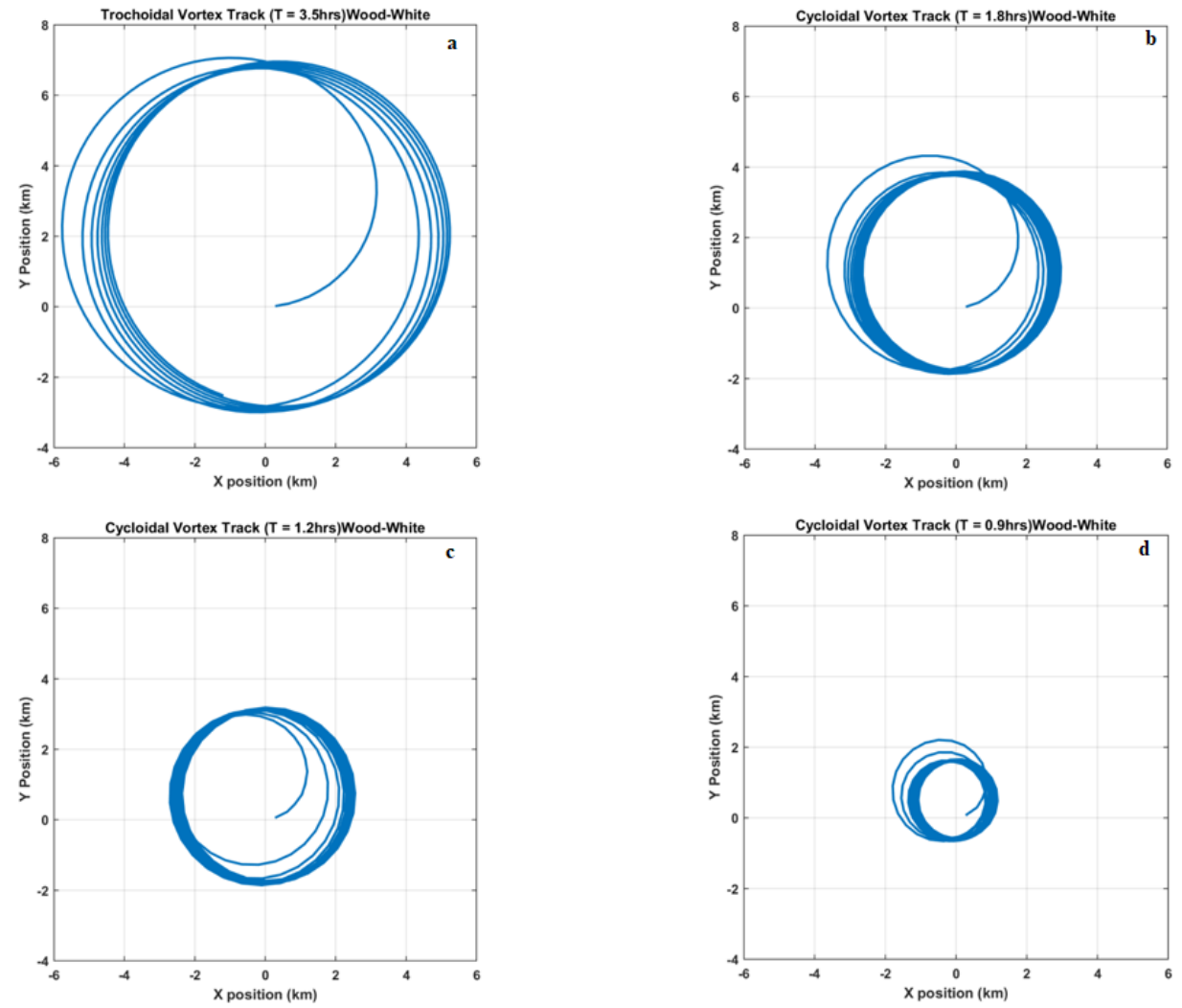

Fig 25. Vortex tracks showing a cyclonic orbit for different specified orbital periods: 3.5 hrs (a), $1.8 \mathrm{hrs} \mathrm{(b),1.2} \mathrm{hrs} \mathrm{(c),} \mathrm{and} 0.87 \mathrm{hrs} \mathrm{(d).}$
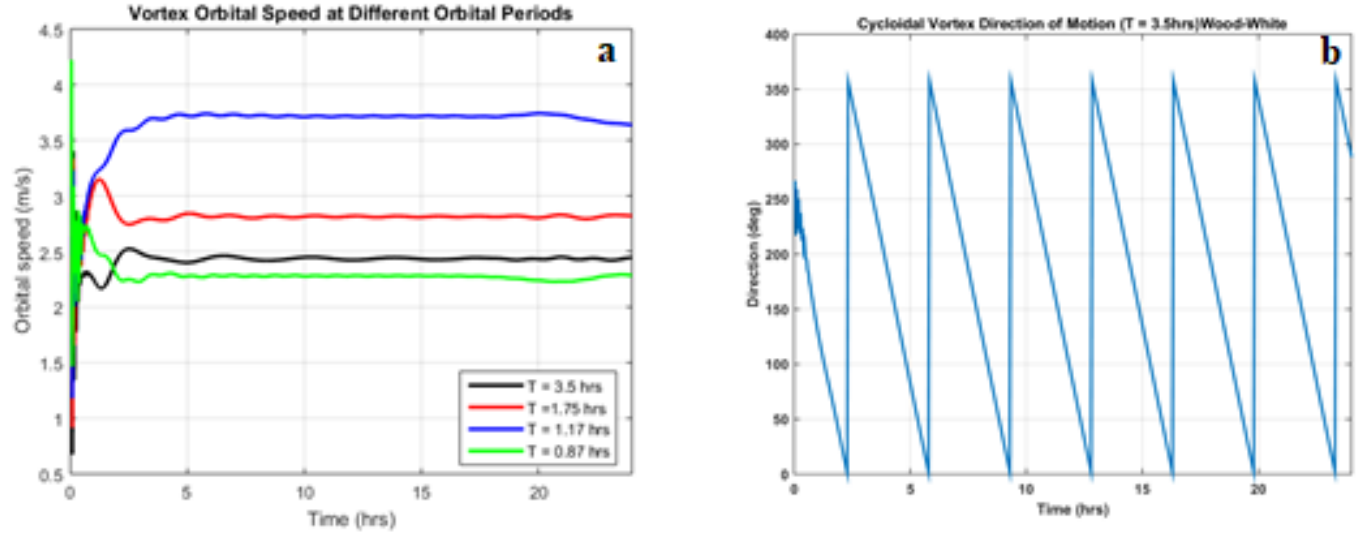

Fig 26. Vortex orbital speeds ranging from $\sim 2.25$ to $3.6 \mathrm{~ms}^{-1}$ for different forcing orbital periods (a), and direction of motion for a 3.5-hr orbital period (b). The latter shows that the vortex center completes nearly 7 orbits. 


\section{Eddy Fluxes}

Vortex Rossby waves transport energy and angular momentum that can change TC structure and intensity. It was noted by NM99 that eddy fluxes are driving the meanvortex flow toward solid-body rotation near the center axis in response to a "wiping out" of the angular velocity deficit inside the eye by the growing wavenumber-1 disturbance. Initially inward-propagating energy packets support an outward geopotential (energy) flux, $\langle u \phi\rangle$, that is balanced by inward angular momentum flux, $\langle u v\rangle$, carried by the waves reflected from the turning point. The scenario is reversed for outward-propagating VRWs (Fig 27). The end result is $\langle u v\rangle$ convergence that accelerates the mean flow $(\partial v / \partial t)$ at the RMW, and $\langle u v\rangle$ divergence that decelerates the mean-flow in the neighborhood of the critical radius (Fig 28). The $\langle u v\rangle$ gradient determines the magnitude of $\partial v / \partial t$, with both boundaries of the waveguide exhibiting the strongest gradients. For $T=3.5 \mathrm{hrs}$ (Fig 28a), the magnitudes of the acceleration and deceleration are nearly identical, which results only in redistribution of vortex mean flow, but no net acceleration. In barotropic conditions, VRW radial propagation causes the vortex spin-up to occur outside the RMW where the initial forcing is prescribed, indicating that eyewall disturbances can lead to storm intensification and size expansion via wave-mean flow interaction (Gao 2016). 

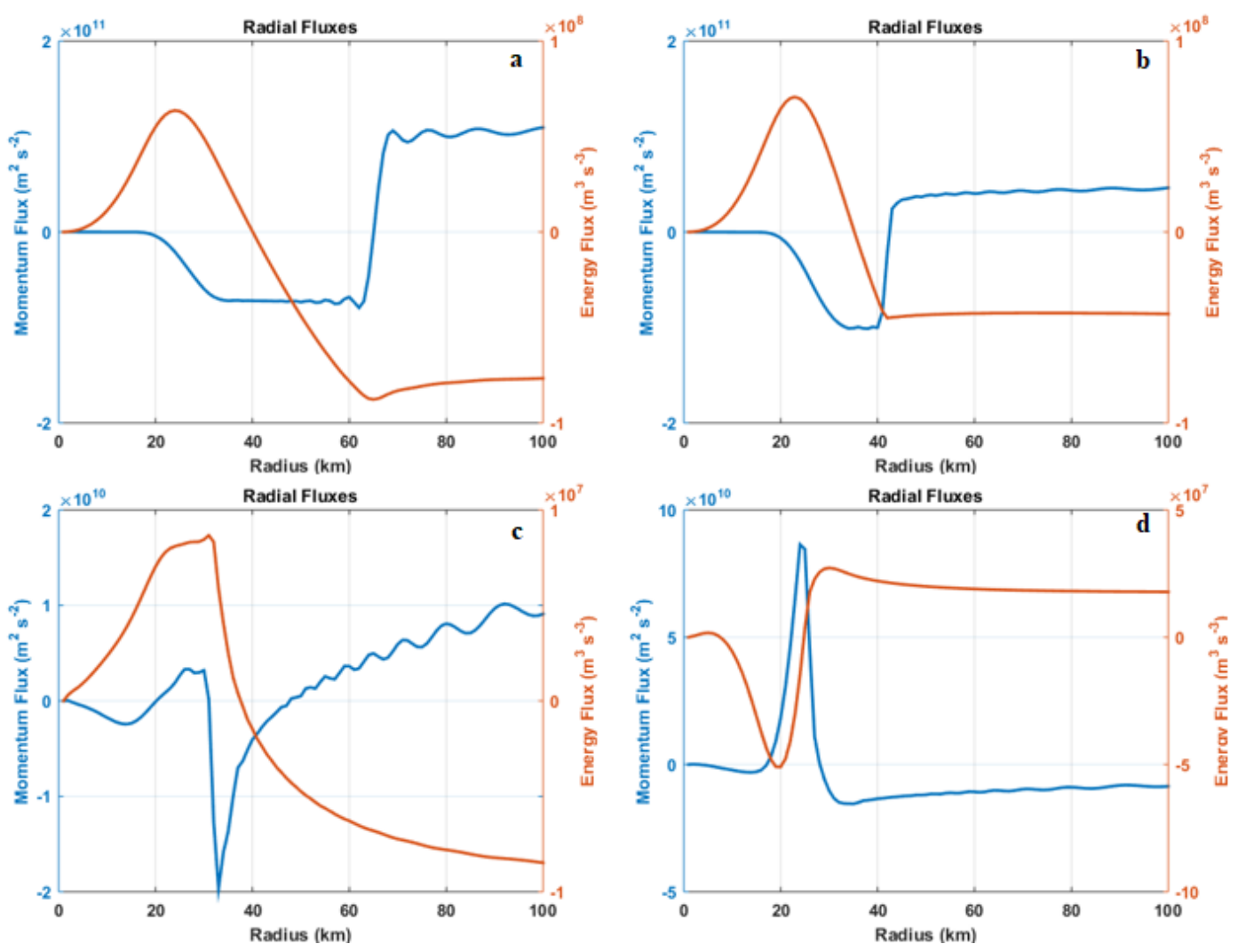

Fig 27. Angular momentum (blue curve) and geopotential fluxes (orange curve) for different specified orbital periods: $3.5 \mathrm{hrs}$ (a), $1.8 \mathrm{hrs}$ (b), $1.2 \mathrm{hrs}$ (c), and $0.87 \mathrm{hrs}$ (d). 

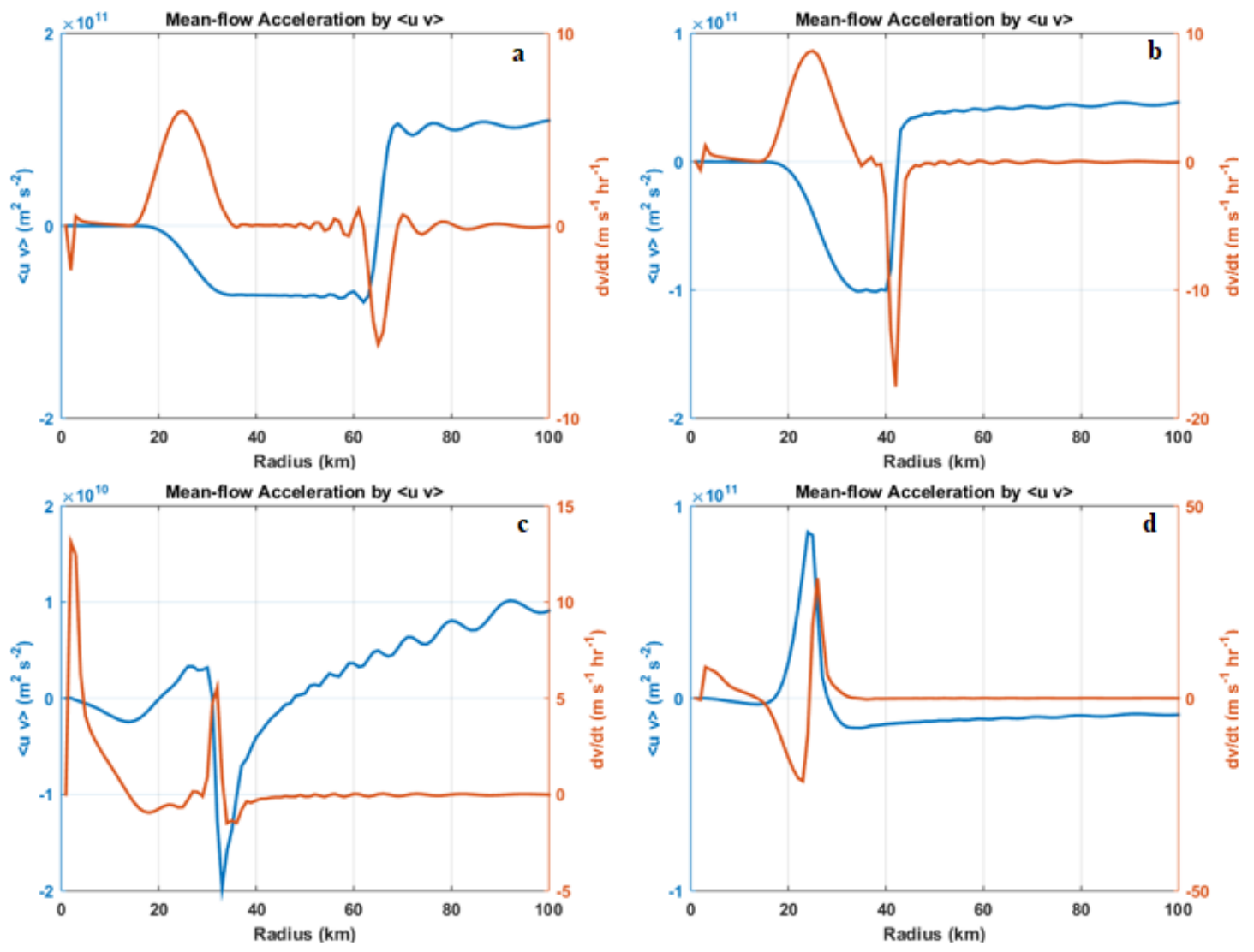

Fig 28. Mean-flow acceleration (orange curve) superimposed with angular momentum flux (blue curve) for different specified orbital periods: $3.5 \mathrm{hrs}$ (a), $1.8 \mathrm{hrs}$ (b), $1.2 \mathrm{hrs}$ (c), and $0.87 \mathrm{hrs}(\mathrm{d})$.

The Inner Waveguide

Wavenumber-1 VRWs propagate upon the mean-vortex radial vorticity gradient within the widest inner waveguide (Fig 29a). In frequency space, the waveguide is bounded by zero frequency (blue) and the frequency of a one-dimensional VRW, $\Omega_{1 \mathrm{D}}$ (red). The latter is the most negative frequency possible for a propagating VRW effectively the cutoff frequency. Vortex Rossby waves obey the dispersion relation (equation 11) so that the wave packets propagate upstream with a negative $\Omega$. In the limit 
of large $k_{r}$, such that the waves become radially short, $\Omega \rightarrow 0$ (11.1). Therefore, outwardpropagating energy is Doppler-shifted to zero frequency, when $\omega$ exactly equals the mean-flow angular velocity $(\mathrm{nV} / \mathrm{r})$, and are absorbed at the critical radius. In the limit of small $k_{r}$ (long waves), $\Omega \rightarrow \Omega_{1 \mathrm{D}}$ (11.2), which is most negative for $n=1$. Inwardpropagating energy is Doppler-shifted to the cutoff frequency, reflected from the turning point and redirected outward toward the critical radius. However, some energy leaks out of both waveguide boundaries to form evanescent tails. Outward from the critical radius, $\Omega>0$, and inward from the turning point, $\Omega<\Omega_{1 \mathrm{D}}<0$.

$$
\begin{gathered}
\lim _{k_{r} \rightarrow \infty} \Omega=\frac{\frac{n}{r} \frac{\partial \zeta}{\partial r}}{k_{r}^{2}+\frac{n}{r^{2}}} \rightarrow 0 \\
\lim _{k_{r} \rightarrow 0} \Omega=\frac{\frac{n}{r} \frac{\partial \zeta}{\partial r}}{k_{r}^{2}+\frac{n}{r^{2}}} \rightarrow r n^{-1} \frac{\partial \zeta}{\partial r}=\Omega_{1 D}
\end{gathered}
$$

The waveguide is defined by a range of $\Omega$ passbands (each corresponding to a value of $\omega$, the propagation frequency relative to the ground) that supports VRW free-propagation in a radial interval between the turning and critical radii: $\Omega_{1 \mathrm{D}}<\Omega<0$ (Fig 29a). However the waveguide narrows as $n$ increases (Fig 29c,d). 

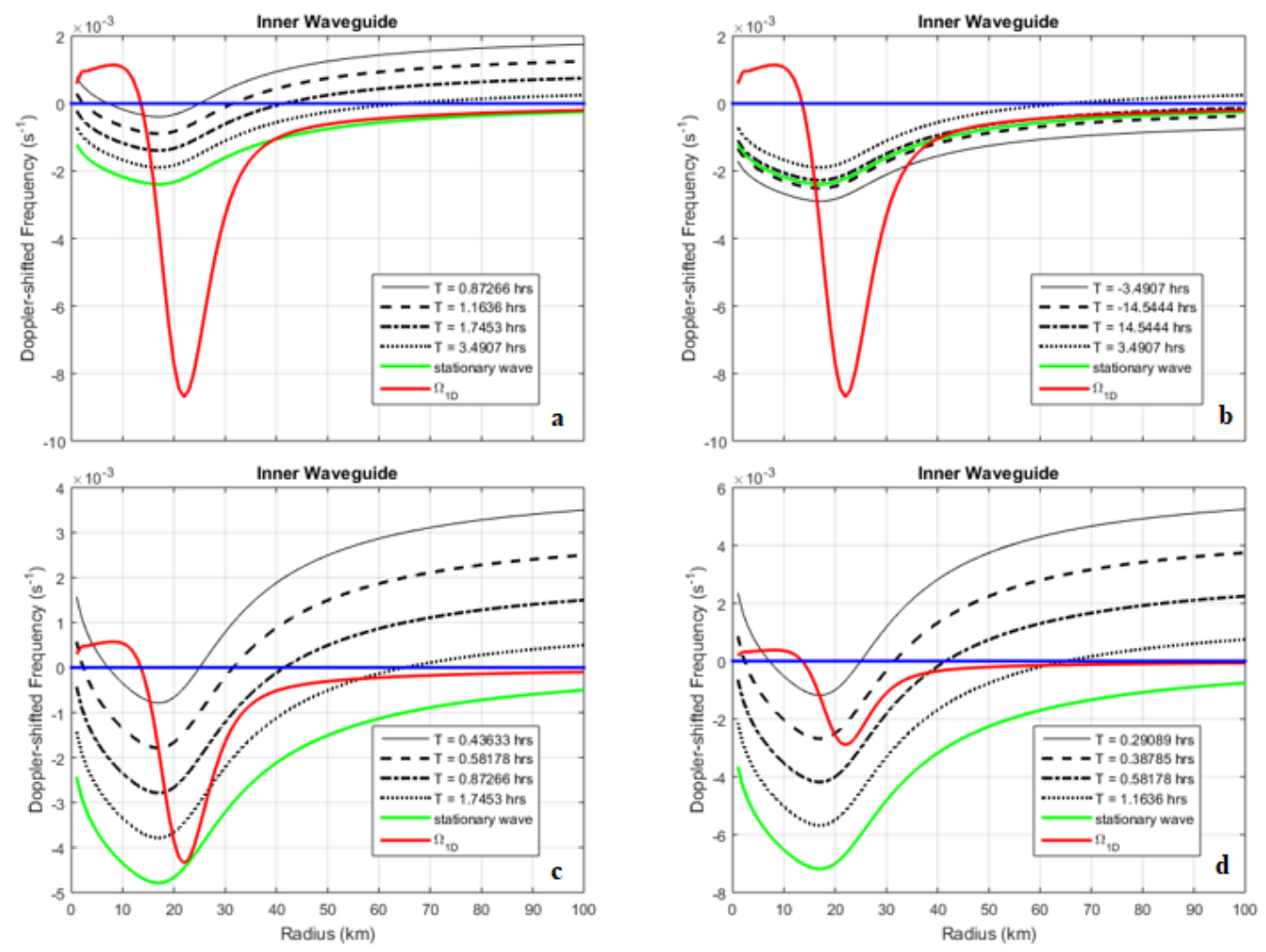

Fig 29. Wavenumber-1 inner waveguide (top row) highlighting that frequency passbands between the cutoff (red curve) and zero frequency (blue curve) represent free-propagating VRWs (a) radially trapped VRWs Doppler-shifted to the cutoff frequency twice (b). Wavenumber-2 (c) and wavenumber-3 (d) inner waveguides are much narrower by comparison.

The specified frequency determines how fast the forcing rotates as a whole and the radial interval for which a chosen VRW can propagate. A slow rotational forcing yields a large critical radius, and a wide passband. Critical radii for $0.25 \omega_{\mathrm{o}} \leq \omega \leq \omega_{\mathrm{o}}$ range between $\sim 25 \mathrm{~km}$ on the high-frequency side and $130 \mathrm{~km}$ on the low-frequency side, whereas the turning point is basically fixed at $\sim 20 \mathrm{~km}$. The sensitivity of the passband's width to changes in $\omega$ also determines the distance over which VRW transports act. Table 1 summarizes the main results from four different specified frequencies. If $\omega$ is sufficiently small, forced VRWs become radially trapped between two turning points (Fig 
29 b), which generally results in the absence of a critical radius. Trapped waves could hypothetically build up energy to large amplitudes through multiple reflections from both boundaries.

Table 1. Vortex motion results and waveguide width for a forcing with different specified frequencies.

\begin{tabular}{|c|c|c|c|c|}
\hline & $\boldsymbol{\omega = 0 . 2 5 \omega _ { \mathbf { o } }}$ & $\boldsymbol{\omega = 0 . 5 0 \omega _ { \mathbf { o } }}$ & $\boldsymbol{\omega = \mathbf { 0 . 7 5 } \boldsymbol { \omega } _ { \mathbf { 0 } }}$ & $\boldsymbol{\omega}=\boldsymbol{\omega}_{\mathbf{0}}$ \\
\hline $\boldsymbol{T}$ (hrs) & 3.5 & 1.74 & 1.16 & 0.87 \\
\hline \# of orbits & 6.9 & 13.8 & 20.7 & 27.6 \\
\hline $\begin{array}{c}\text { Waveguide } \\
\text { width (km) }\end{array}$ & 50 & 32 & 19 & 15 \\
\hline $\begin{array}{c}\text { Orbital speed } \\
\left(\mathbf{m s}^{-\mathbf{1}}\right)\end{array}$ & 2.42 & 2.81 & 3.65 & 2.29 \\
\hline $\begin{array}{c}\text { Orbital radius } \\
(\mathbf{k m})\end{array}$ & 4.86 & 2.82 & 2.45 & 1.14 \\
\hline
\end{tabular}

\section{VRW Trapping}

In a low-frequency cyclonic passband (e.g., $T=14.5 \mathrm{hrs}$ ), $\Omega$ intersects $\Omega_{1 \mathrm{D}}$ outward from $R_{Q}$. The passband is composed of three main regions: 1) trapped waves between an inner and outer turning point, with continuous wave reflection from both boundaries; 2) evanescent waves where some energy leaks past the outer turning point; 3) inner waveguide re-entry between a second turning point and an, outer critical radius. Each of the regions has small radial intervals so that the VRWs are even more confined. If $\omega=0$, then the waves are stationary. The passband for an anticyclonic $\omega$ (e.g., $T=$ $-3.5 \mathrm{hrs}$ ) does not have a critical radius; instead the passband represents trapped waves between inner and outer turning points and evanescent waves outward from the outer turning point that never re-enter the waveguide. Therefore, VRWs with $\omega<0$ are incapable of free propagation. Table 2 lists $\Omega$ for wavenumber-1 VRWs at various states, 
where $V / r$ is the mean-flow angular velocity, $\Omega_{1 \mathrm{D}}{ }^{\text {in }}$ and $\Omega_{1 \mathrm{D}}{ }^{\text {out }}$ are the cutoff frequencies at the inner and outer turning points, respectively.

Table 2. Wavenumber-1 VRW frequencies at different states.

\begin{tabular}{|c|c|}
\hline VRW state & Doppler-shifted Frequency \\
\hline Freely propagating & $\Omega_{1 \mathrm{D}}<\Omega<0$ \\
\hline Absorbed & $\Omega=0(\omega=V / r)$ \\
\hline Reflected & $\Omega=\Omega_{1 \mathrm{D}}$ \\
\hline Evanescent & $\Omega<\Omega_{1 \mathrm{D}}<0$ \\
& $\Omega>0$ \\
\hline Radially trapped & $\Omega_{1 \mathrm{D}}$ in $<\Omega<\Omega_{1 \mathrm{D}}{ }^{\text {out }}$ \\
\hline Stationary & $\Omega=-V / r(\omega=0)$ \\
\hline
\end{tabular}

Vorticity results for "trap-inducing" frequencies consists of a wavenumber-1 dipole with vorticity filaments radiating away from $R_{Q}$. Since only a limited amount of evanescent wave energy reaches the critical radius for $T=14.5 \mathrm{hrs}$, interlocking trailing spirals do not form (Fig 30a,b). Instead a vorticity wake extends from both sides of the forced dipole, outward to the edge of the domain. Therefore unimpeded VRW propagation appears necessary for spiral rainband development. The corresponding $\psi$ simply illustrates the two familiar, broad outer gyres superimposed with an inner pair of smaller, convectively forced gyres (Fig 30c,f). 

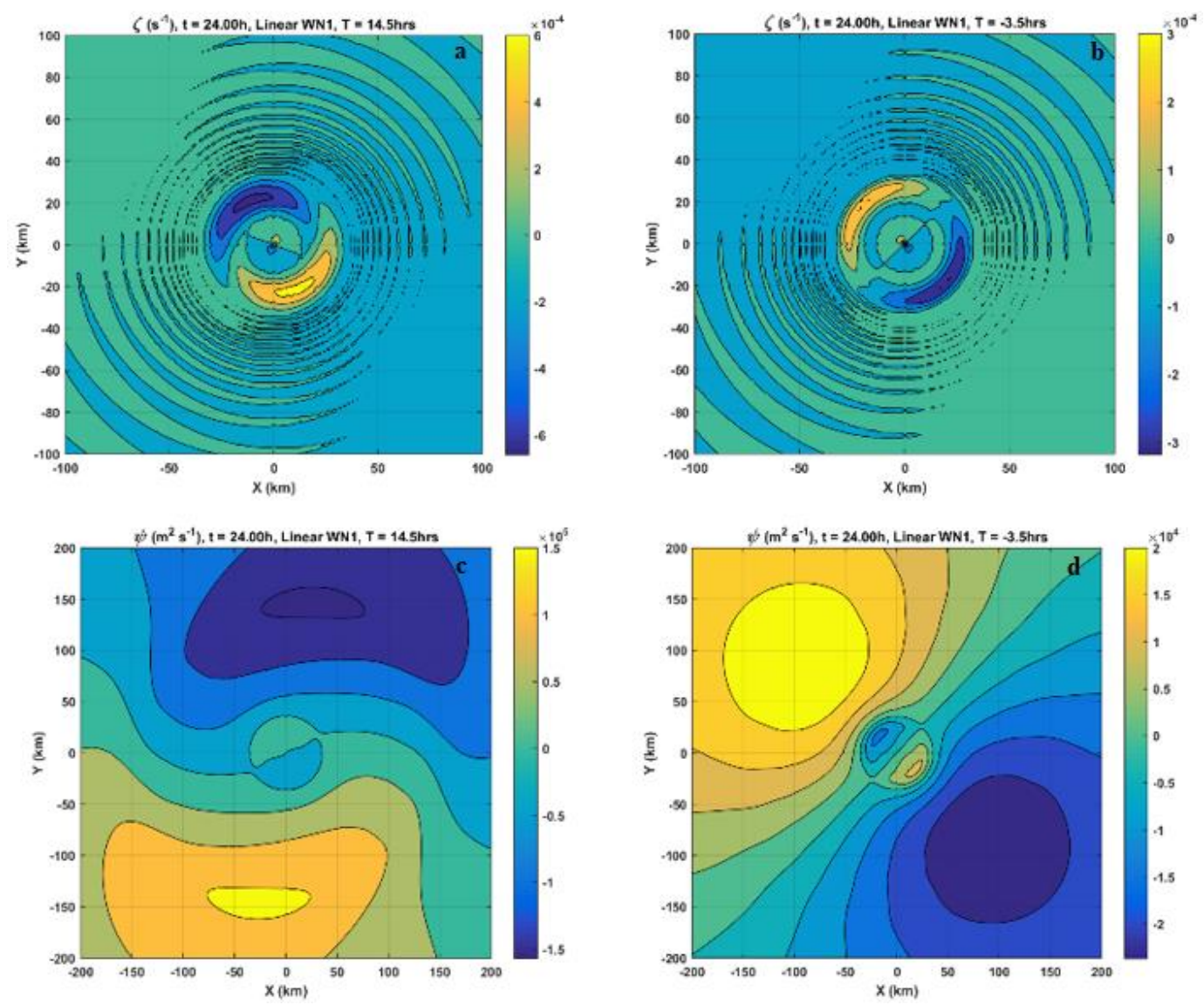

Fig 30. Vorticity $(\mathrm{a}, \mathrm{b})$ and streamfunction contour plots $(\mathrm{c}, \mathrm{d})$ for a 14.5 -hr orbital period (left column) and a $-3.5-h r$ orbital period (right column).

Forcing with $T=14.5$ hrs $(\sim 1.6$ orbits $)$ produces a trochoidal track with a 1.4 $\mathrm{ms}^{-1}$ mean orbital speed, where the vortex center is displaced up to $20 \mathrm{~km}$ from the original starting point (Fig 31a,b). Negative specified frequencies result in the vortex following a clockwise track that initially spirals anticyclonically outward with a $\sim 1.75$ $\mathrm{ms}^{-1}$ mean orbital speed (Fig 31c,d). Although in nature eyewall convection rotates downstream with the mean cyclonic winds, the vortex center's tendency to follow the forcing's rotation is evident. By comparison, Willoughby (1988) mentioned that with no critical radius at anticyclonic frequencies, the source-sink pair forces little vortex motion. 

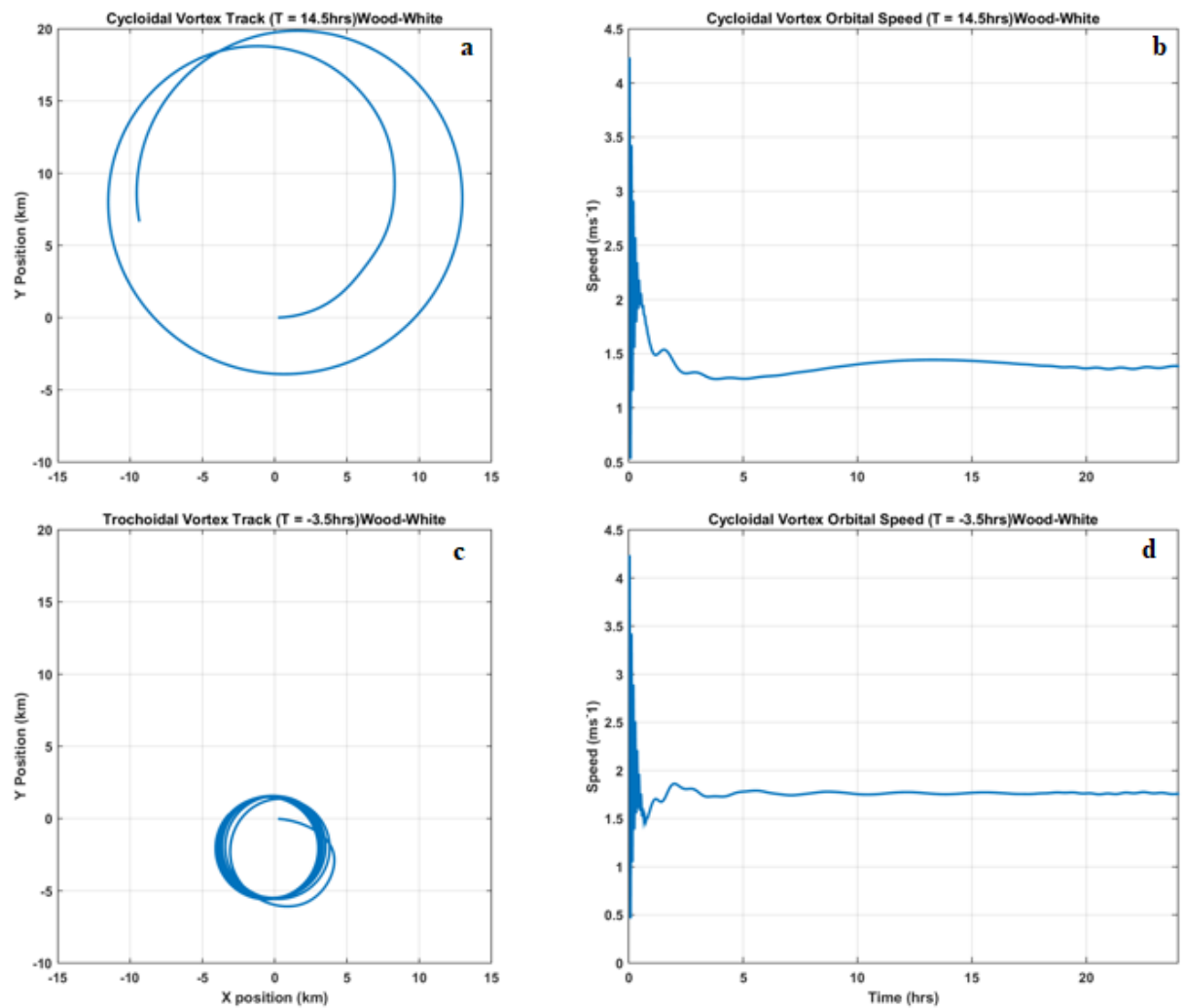

Fig 31. Vortex tracks (left column); orbital speed (right column); Top row: 14.5-hr forcing orbital period; bottom row: -3.5 -hr forcing orbital period.

Eddy flux budgets for trap-inducing frequencies show a positive peak in $\langle u \phi\rangle$ at the RMW and strongly negative $\langle u \phi\rangle$ beyond the vortex inner core (Fig 32a,c). For $T=$ $14.5 \mathrm{hrs}$, there is a low-amplitude inward $\langle u v\rangle$, and a strong gradient from $\sim 140-180 \mathrm{~km}$, resulting in a greater $\partial v / \partial t$ at the RMW than the deceleration at the critical radius where some of the evanescent wave energy re-enters the waveguide (Fig 32b). For $T=-3.5$ hrs, $\langle u v\rangle$ is unstable, consisting of oscillations with increasing amplitude at greater distances from the vortex center. The trapped VRWs in the $18-35 \mathrm{~km}$ radial interval produce weak $\partial v / \partial t$ amplitudes with a minimum near the inner turning point and maximum near the outer turning point (Fig 32d). Small values of $\langle u \phi\rangle$ and $\partial v / \partial t$ offer no support for the 
hypothesis that continuous wave reflection between two turning points is a possible vortex intensification mechanism. However, radially trapped VRWs do reveal that freewave propagation is important for producing trailing spirals at the critical radius, and shows that the vortex center can perform anticyclonic wobbles if the forcing rotates with a negative specified frequency.
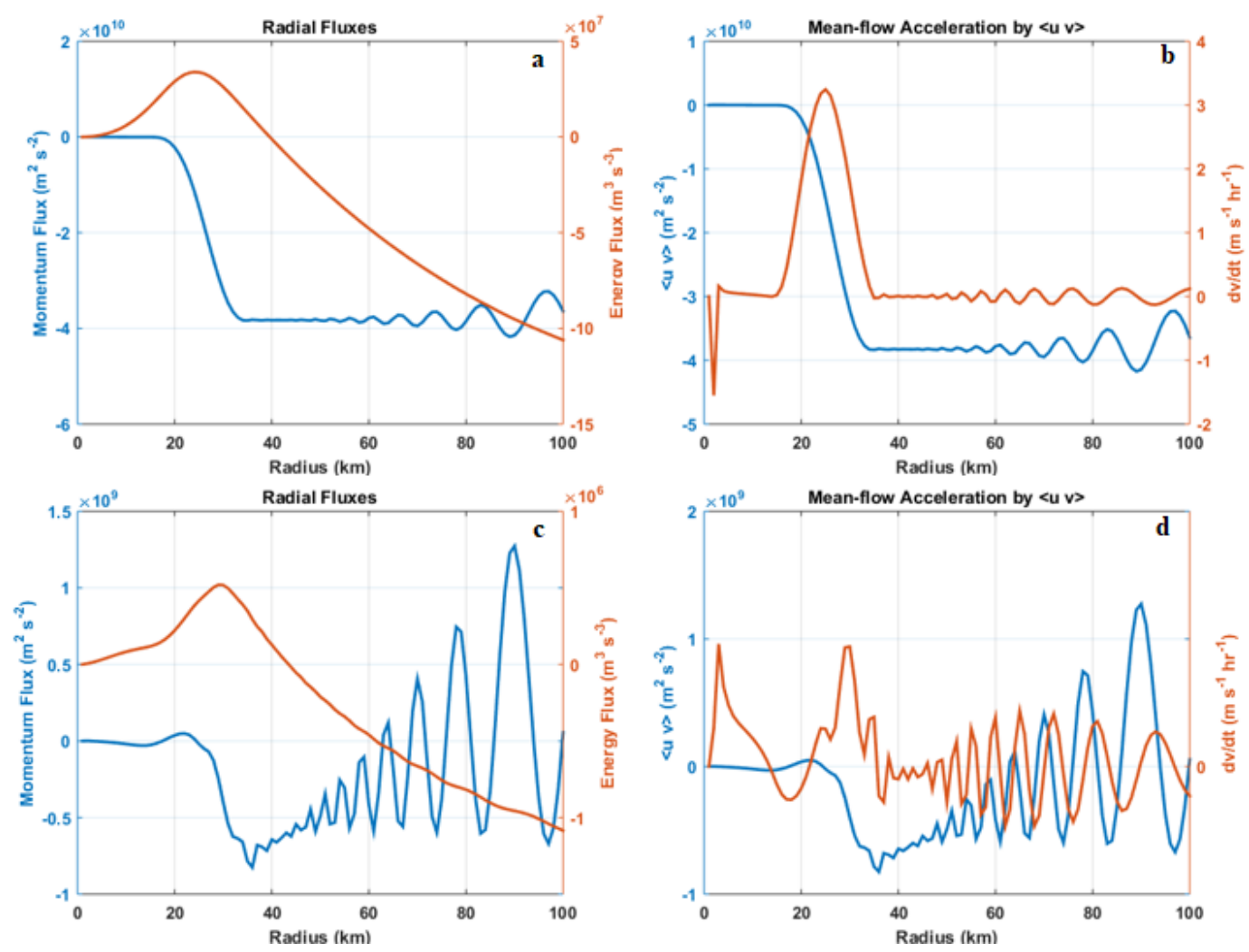

Fig 32. Radial fluxes $(\mathrm{a}, \mathrm{c})$ and mean-flow acceleration induced by angular momentum flux $(\mathrm{b}, \mathrm{d})$ for a $14.5-\mathrm{hr}$ orbital period (top row) and a -3.5 -hr orbital period (bottom row). 
Summary

A simple, barotropic, nondivergent model provides useful insight into the dynamics of wavenumber-1 vortex Rossby waves primarily in the context of vortex motion and innerwaveguide propagation. Vortex Rossby waves are excited by a mass source-sink pair that rotates with a specified frequency in the eyewall and appear as vorticity gyres surrounded by an outer symmetric ring of highly filamented, tightly wound trailing spirals in the neighborhood of the critical radius, resembling observed outer rainbands. Between the ring and forced gyres are numerous vorticity filaments, consistent with outward wave propagation. Outward from the ring is a low-amplitude wake that aligns with the mean vortex's direction of motion and slipstream as the storm moves through a low-vorticity environment. The wake is also associated with some wave energy leaking out of the waveguide and exponentially decaying away.

The corresponding streamfunction consistently shows inner gyres forced by the mass source-sink pair superimposed with larger outer gyres forced by storm motion. The latter arise from a secondary instability as a result of the vorticity gradient increasing between the outward spiraling vortex center and eyewall. The cyclonically rotating inner gyres advect vorticity which causes the low-vorticity vortex center to be displaced in the direction of advection and causes trochoidal motion, which is dependent on the specified rotation frequency. If the forcing rotates rapidly, orbital speed generally increases but the orbital radius become smaller. The eddy fluxes show outward energy flux near the forcing radius and an inward angular momentum flux convergence. The latter accelerates the mean flow at the radius of maximum wind. Additionally, angular momentum flux 
divergence occurs at the critical radius, which decelerates the mean flow there. The mean flow is subsequently redistributed and convection is localized so that trailing spirals form.

In the inner waveguide, vortex Rossby waves propagate upon the negative meanvortex radial vorticity gradient. The intersection of the Doppler-shifted frequency with zero and the cutoff frequencies determine the radial interval of free propagation. The inner and outer edges of the radial interval are the turning point and critical radius. Frequency passband width is determined by the propagation frequency chosen. If the frequency is high, the passband is narrower, resulting in a critical radius closer to the center. The larger the critical radius, the greater the wave-induced displacement of the vortex-center. Initially outward-propagating group velocity slows as the waves approach the critical radius and become radially short to form trailing spirals. Ultimately the waves are Doppler-shifted to zero frequency and absorbed at the critical radius. Inwardpropagating vortex Rossby waves become radially longer as the energy is Dopplershifted to the cutoff frequency. Waves reflected from the turning point then propagate outward past the forcing radius to the critical radius to be absorbed as well. Some wave energy leaks past the critical radius and inward from the turning point, as evanescent tails.

If a frequency passband intersects the cutoff frequency twice, the waves are radially trapped between two turning points such that the wave energy continuously reflects from both boundaries. In order for forced waves in the mean vortex to be trapped, the forcing must rotate with either very low cyclonic frequencies or any anticyclonic frequency. The former may still contain a large critical radius for energy that re-enters the waveguide from the evanescent region. However, the leaked wave 
energy is not strong enough to produce the tightly wound trailing spirals. Therefore, freewave propagation is necessary to produce trailing spirals. An anticyclonic frequency on the other hand, never includes a critical radius, but induces clockwise vortex-track orbits. It was initially hypothesized that trapped waves had a role in vortex intensification by energy growth through continuous reflection within a narrow radial interval, but experimentation yielded no supporting evidence.

The results from Chapter 3 also highlight notable similarities and differences between forced wavenumber-1 and wavenumber $\geq 2$ vortex Rossby waves. For all wavenumbers, trailing spirals form in the neighborhood of the critical radius. Near the center, eddy fluxes of angular momentum and wave-energy are inward and outward, respectively. One important distinction, however, is the inner waveguide width decreases with increasing tangential wavenumber. Wavenumber 1 yields the radially widest waveguide, which increases the distance of wave-energy and angular momentum transport by forced waves. Narrower waveguides require the forcing to rotate with a specified frequency closer to the orbital frequency at the radius of maximum wind to support freewave propagation. A much larger range of rotation frequencies relative to the ground can fit within the wavenumber-1 vortex Rossby waveguide which enables greater interval for wave propagation, such that trailing spirals can form at large critical radii.

Another key difference is that vorticity advection between wavenumber-1 streamfunction gyres results in vortex motion. Forced wavenumber $\geq 2$ perturbations are a wavetrain of eddies with alternating polarity, advected downstream by the mean swirling flow. The adjacent gyres' sunflower-like orientation produces a balanced inward-outward vorticity exchange between the vortex's eye and eyewall, resulting in zero net vorticity 
advection across the center. The high-wavenumber flow pattern therefore precludes vorticity-advection-induced vortex motion but is consistent with observed eyewall mesovortices. Nevertheless, wavenumber $\leq 2$ vortex Rossby waves are the best representation of the waves' dynamics because the waveguides are sufficiently wide to support propagation and yield results most consistent with observations.

Studying vortex Rossby waves excited by a continuous eyewall forcing rotating at different specified frequencies has proven instrumental in advancing understanding of the waves' influences on tropical cyclone motion and propagation dynamics in the widest possible waveguide, in addition to yielding clarity on vortex structure evolution and eddy flux budgets. However, experimental changes to other initialized forcing parameters require further attention because observed tropical cyclone convection is known to fluctuate in position, coverage, and intensity. Therefore, sensitivity studies of forcing radius and width, episodic forcing, and beta-plane simulations are the focus in the next chapter. 


\section{CHAPTER IV. FORCING SENSITIVITY STUDIES AND BETA-PLANE SIMULATIONS}

\section{General Overview}

Chapter 3 established that wavenumber-1, rotating, "convective" forcing imposed at the RMW, excited VRWs that propagated away from the forcing radius, $R_{Q}$ within an inner waveguide. The waves produced a tightly wound interlocked ring of filamented vorticity at the critical radius, converged angular momentum inward to accelerate the mean flow at the RMW, and forced trochoidal vortex motion in slow, small-scale orbits. The wide, wavenumber-1 inner waveguide is defined by the vortex's negative radial vorticity gradient that supports VRW propagation with a large range of frequencies between zero and the VRW cutoff frequency. The forcing's rotation frequency, $\omega$, was specified as a fraction of the vortex orbital velocity at the RMW. Critical radii, vorticity ring size, and orbital speed and radius were controlled by $\omega$.

Despite key findings, questions remain about the vortex response to changes in the model's forcing parameters that include convective forcing radius $\left(R_{Q}\right)$, width $\left(W_{Q}\right)$, and amplitude, as well as $\beta$-plane forcing. Individual sets of sensitivity studies will be presented in Chapter 4, beginning with shifting $R_{Q}$ so that wave excitation is strongest at radii inward or outward from the RMW. Adjusting $R_{Q}$ can be likened to a sheared TC in which the convection can be displaced from the center, or observed convective bursts that occur away from the eyewall. Secondly, $W_{Q}$ is halved and doubled while imposed at the RMW. The next set of experiments involve episodic forcing where the amplitude is 
reduced or increased halfway through 24-hr simulations to compare with continuous forcing and determine how wavenumber-1 VRW propagation and vortex motion are affected. The motivation for $W_{Q}$ and amplitude sensitivity studies is that observed convection often fluctuates in both coverage and intensity. Furthermore, the sensitivity studies should verify MK97's findings on the excitement of VRWs near the RMW resulting in mean wind acceleration at the forcing locus, and radially broader forcing yielding a stronger response. Final simulations add the $\beta$-effect to the original convective forcing to compare with vortex motion on an $f$-plane.

Forcing Sensitivity Studies

Forcing Radius and Width

In the first set of sensitivity studies, the $10-\mathrm{km}$ wide convective forcing is imposed $15 \mathrm{~km}$ from the vortex center (near the turning point of the waveguide) so that the outer edge of the forcing lies exactly at the RMW. In the interest of simplicity, all other parameters remain unchanged and only VRWs propagating with $T=3.5 \mathrm{hrs}$ are considered. The forced dipole is located just inside the vortex's eyewall and rotates with $\omega$ (Fig 33a). Excited VRWs propagate mostly outward from the tightly rotating asymmetries, to form an outer ring of filaments at the $\sim 60-\mathrm{km}$ critical radius (Fig 33c). Vortex motion consists of a trochoidal track with a small orbital radius at a $\sim 0.9 \mathrm{~ms}^{-1}$ mean orbital speed because there is less total forcing as a result of the reduced circumference of the forcing annulus (Fig 34a,c). Since the dipole is located inside the eye, VRW outward-propagation distance is greater than when $R_{Q}=\mathrm{RMW}$. 
As in Chapter 3, the VRWs converge angular momentum inward and the critical radius is the locus of $\langle u v\rangle$ divergence. The most important result is that mean-flow acceleration $(\partial v / \partial t)$ peaks inside the RMW instead of at the eyewall, consistent with the findings of Wang (2002b), and Chen and Yau (2003) where VRWs accelerated tangential winds directly in the eye. However, the present results here do not show deceleration at the RMW that acts to prevent vortex intensification. Instead, deceleration occurs in the neighborhood of the critical radius while the RMW experiences approximately zero acceleration (Fig 34e). Does the acceleration of the mean flow inside the RMW imply eyewall contraction? If so then imposing the dipole inward may be a plausible mechanism for intensification.

Shifting $R_{Q}$ outward to $35 \mathrm{~km}$ causes the forcing's inner edge to be exactly at the RMW. Forced asymmetries consist of a wavenumber-1 dipole surrounded by two rings of tightly wound, interlocked filaments (Fig 33b,d), that tentatively resembles a triple eyewall structure (e.g., Fig 33f). The inner ring's radius matches the outer edge of the forcing, and the outer ring coincides with the critical radius. The inner ring may be a standing wave reflected over a wider annulus. Nevertheless, numerous filaments appear between the rings, consistent with outward VRW propagation from $R_{Q}$. A large $R_{Q}$ enables excited waves to propagate inward over a longer distance before the energy Doppler-shifts to the cutoff frequency. However, outward radial propagation decreases because $R_{Q}$ is closer to the critical radius. Forced motion has a larger orbital radius with a mean speed orbital speed of $\sim 5.4 \mathrm{~ms}^{-1}$ because there is more total forcing in the system (Fig 34b,d). Just as in the previous model experiment, peak $\partial v / \partial t$ is at the shifted $R_{Q}$, 
instead of the RMW. The magnitude of negative $\partial v / \partial t$ at the critical radius, however is much larger (Fig 34f).
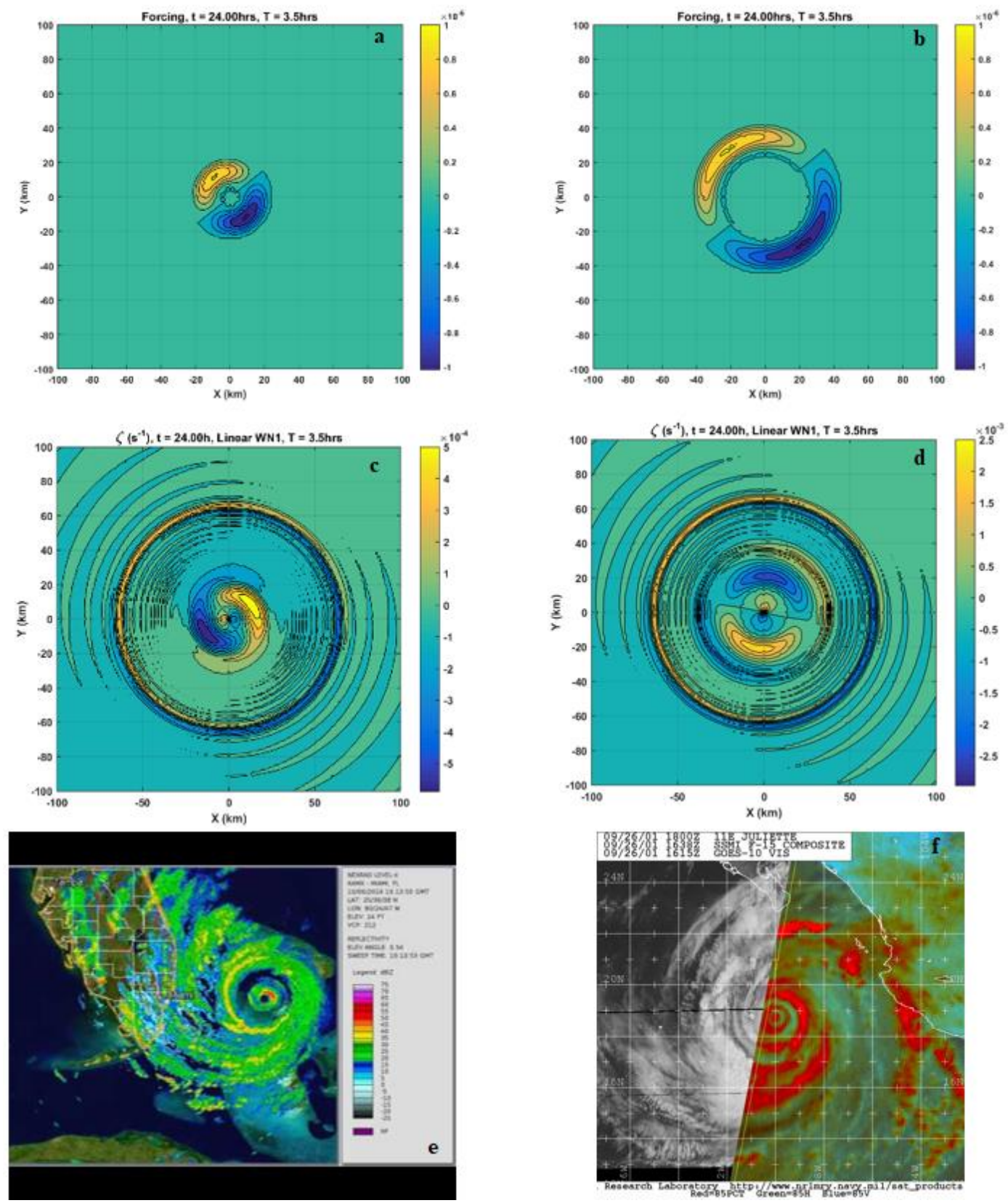

Fig 33. Forcing $(a, b)$ and vorticity $(\mathrm{c}, \mathrm{d})$ contour plots with comparative images of Hurricane Matthew, 2016 (e) and Hurricane Juliette, 2001 (f) for a 15-km forcing radius (left column) and a $35-\mathrm{km}$ forcing radius (right column). 

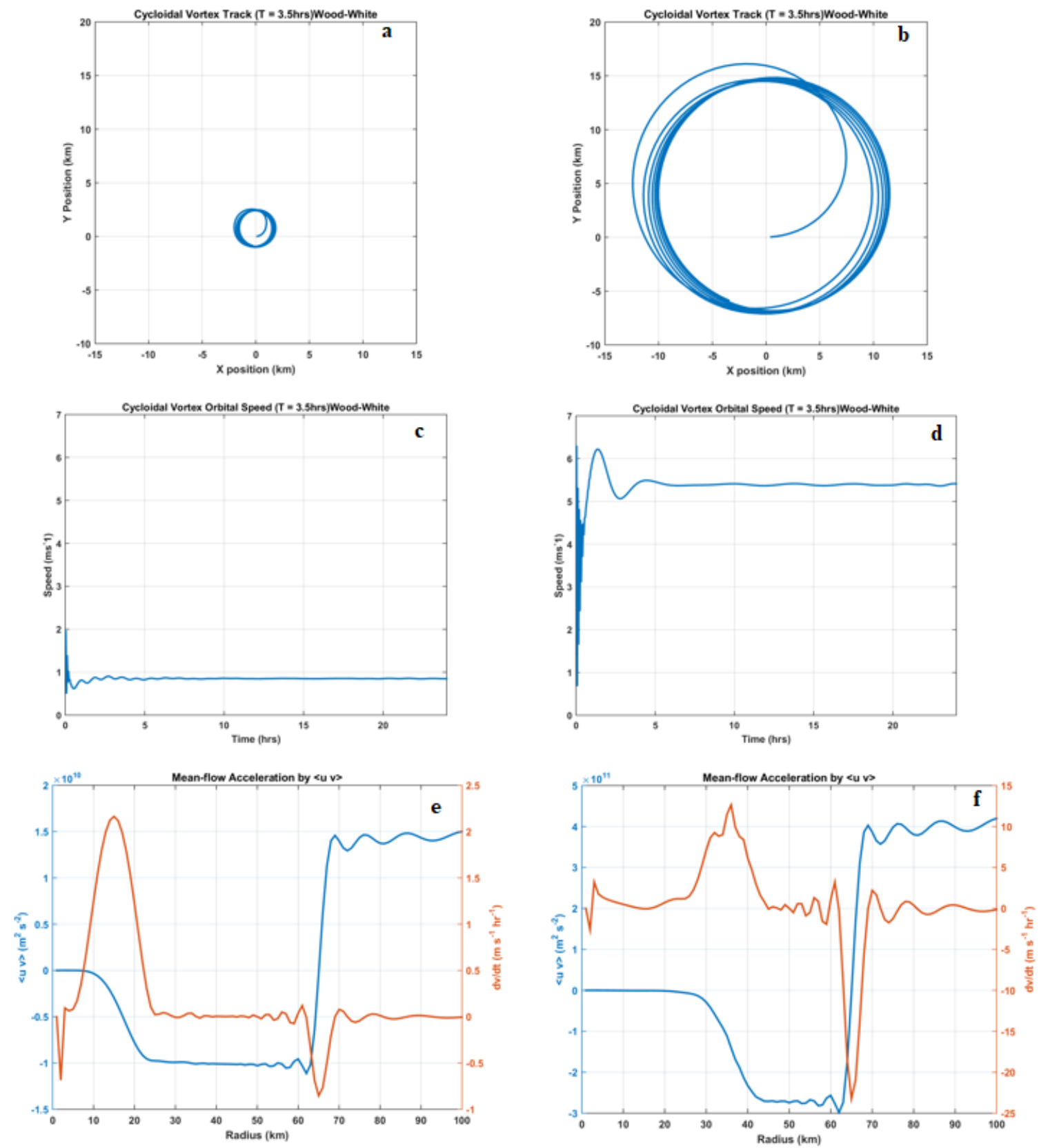

Fig 34. Vortex track (a,b), orbital speed (c,d), and mean-flow acceleration induced by angular momentum fluxes (e,f) for a $15-\mathrm{km}$ forcing radius (left column) and a $35-\mathrm{km}$ forcing radius (right column).

An alternative to displacing forcing radius, $R_{Q}$ from the RMW, is adjusting the width, $W_{Q}$ because area covered by observed eyewall convection varies. Narrowing $W_{Q}$ to $5 \mathrm{~km}$ produces radially thinner asymmetries (Fig 35a,c), smaller track, and orbital 
radius at slower speeds (Fig 36a,c). Angular momentum flux budgets remain nearly the same as in the default model run, with $\partial v / \partial t$ peaking at the RMW but with a lower amplitude. However, the radial width of $\partial v / \partial t$ matches $W_{Q}$ (Fig 36e). Widening the forcing width to $20 \mathrm{~km}$, on the other hand, creates broader asymmetries (Fig 35b,d), and a larger track orbital radius at faster speeds (Fig 36b,d). Mean-flow acceleration peaks at the RMW, but has a wider radial extent (Fig 36f). Therefore the most significant result from the width sensitivity studies is that there is a positive correlation between $\mathrm{W}_{\mathrm{Q}}$ and the radial widths of $\partial v / \partial t$. 

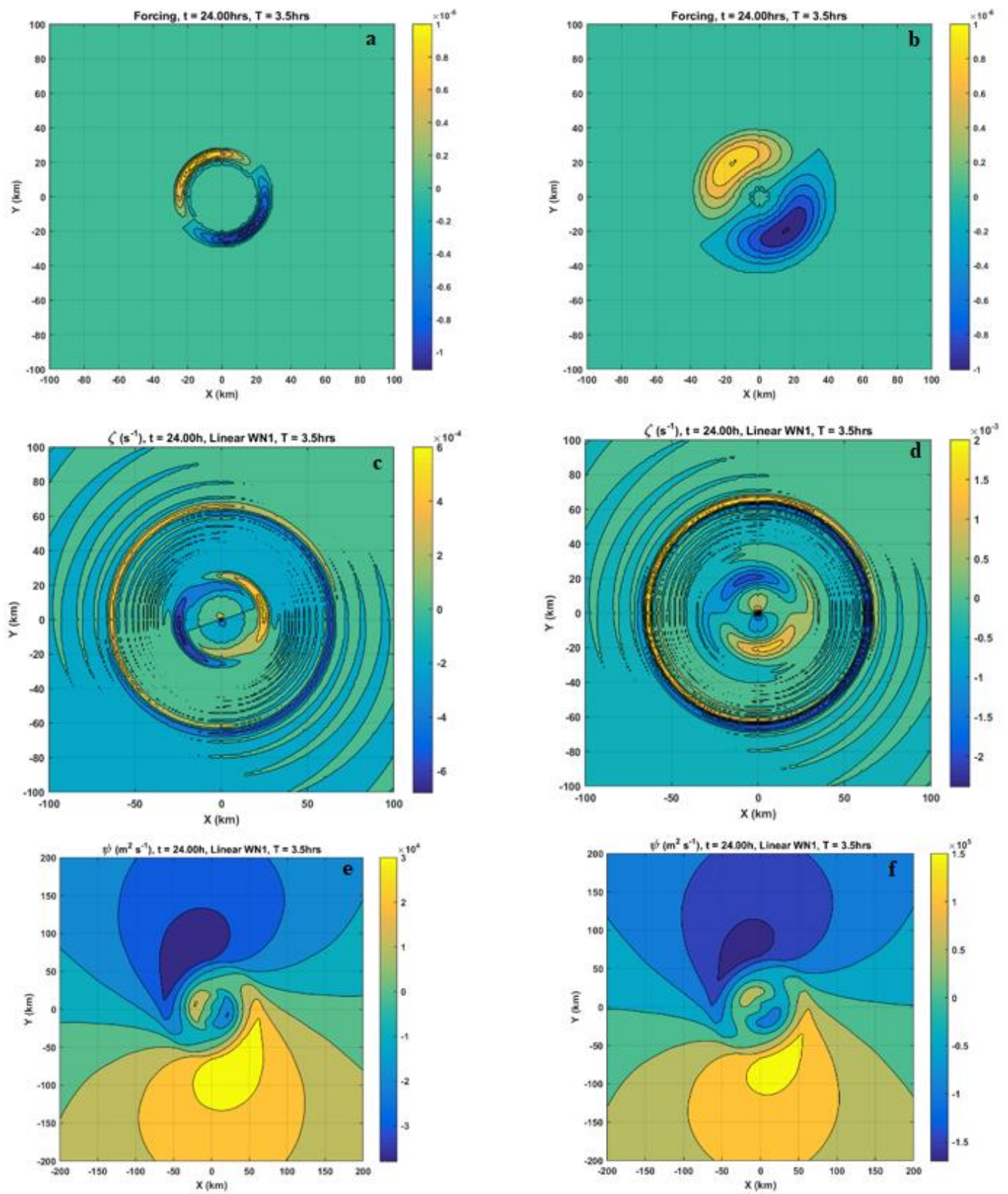

Fig 35. Forcing $(\mathrm{a}, \mathrm{b})$, vorticity $(\mathrm{c}, \mathrm{d})$, and streamfunction $(\mathrm{e}, \mathrm{f})$ contour plots for a 5-km forcing width (left column) and a 20-km forcing width (right column). 

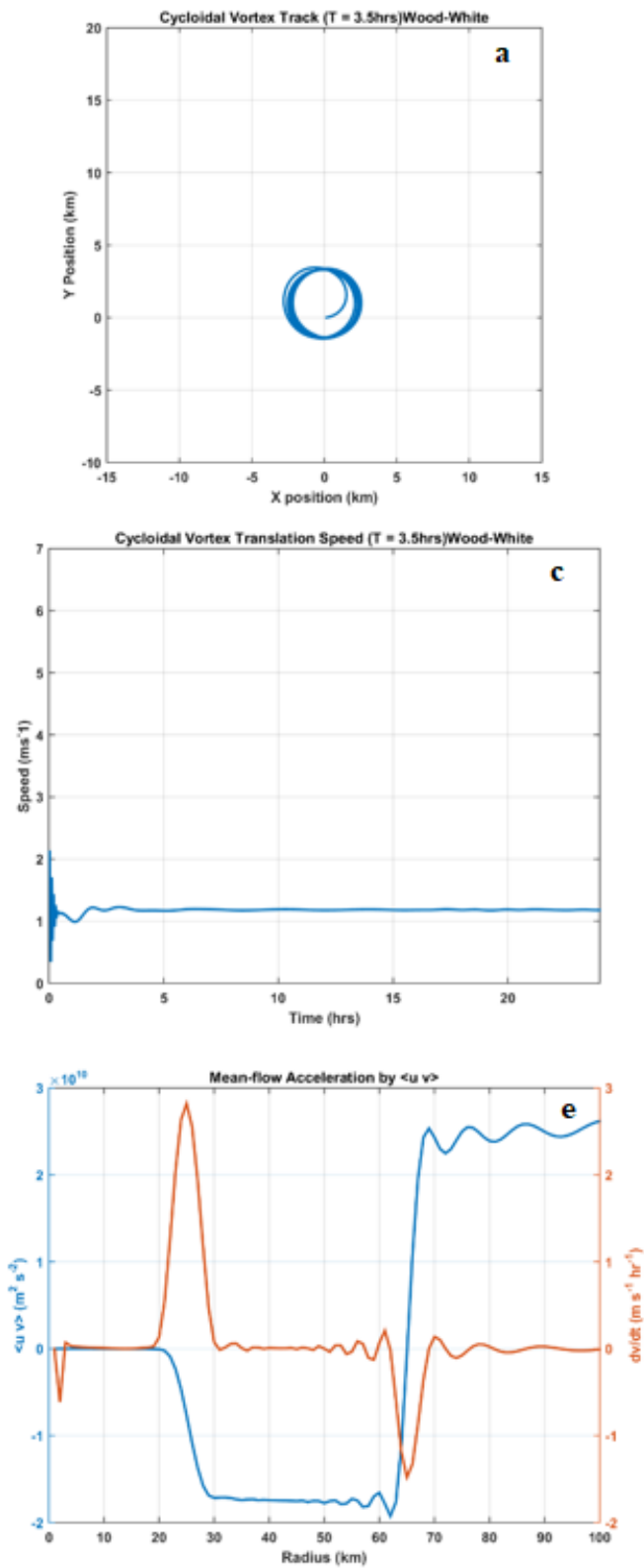
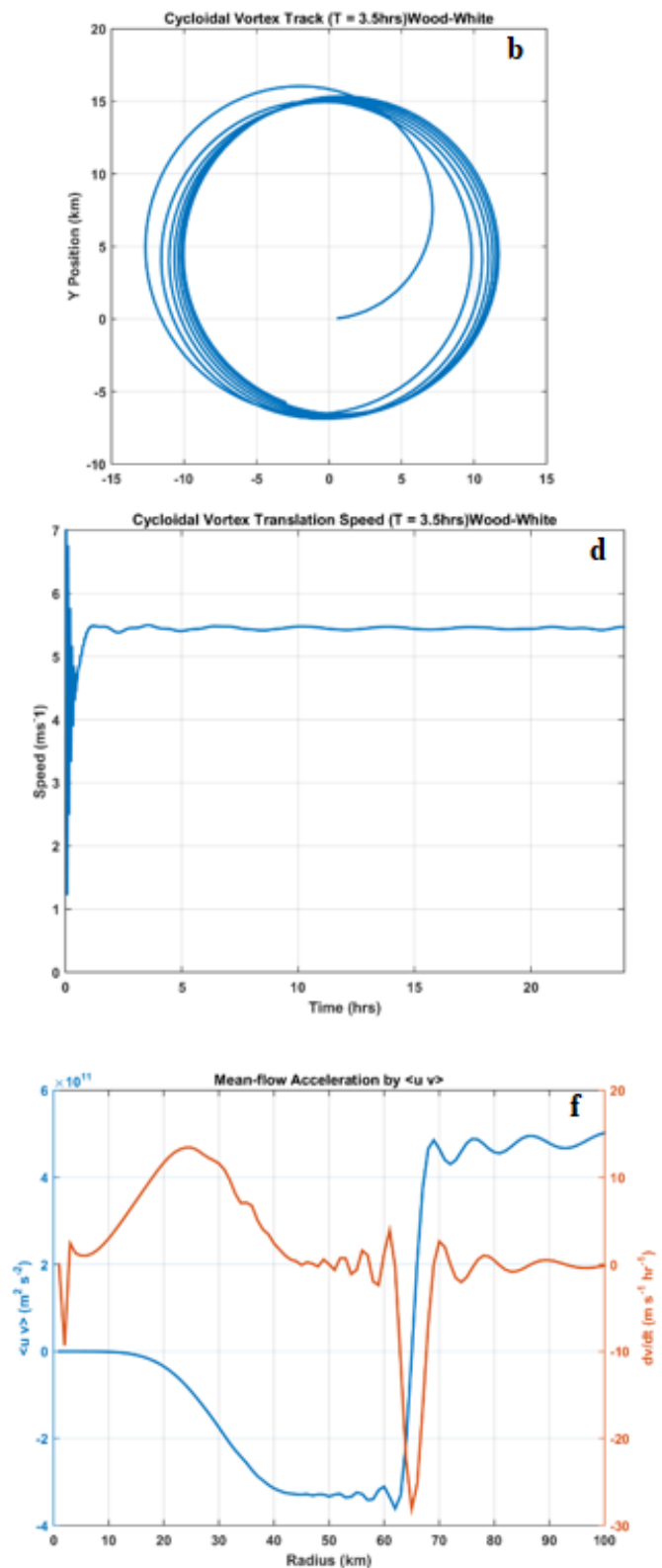

Fig 36. Vortex track (a,b), orbital speed (c,d), and mean-flow acceleration induced by angular momentum fluxes (e,f) for a 5-km forcing width (left column) and a 20-km forcing width (right column).

Varying $R_{Q}$ and $W_{Q}$ generally show that VRW-induced $\partial v / \partial t$ is the most sensitive model result. Mean-flow acceleration consistently peaks at $R_{Q}$; whereas radial interval of $\partial v / \partial t$ increases with $W_{Q}$. Moreover, the vortex motion is faster for a wider forcing, 
simply because the same intensity is spread over a bigger area. Table 3 summarizes the main findings of the forcing sensitivity studies, where $R_{\partial v / \partial t}$ is the radius of peak meanflow acceleration. The next sub-section focuses on episodic forcing where the model is reinitialized 12 hrs into the simulation by reducing or increasing the forcing amplitude. A primary reason for conducting amplitude sensitivity tests is that observed TC convection often fluctuates (e.g., diurnal pulses; Dunion et al. 2014).

Table 3. Vortex motion and mean-flow acceleration results for sensitivity studies on forcing radius and width.

\begin{tabular}{|c|c|c|c|c|}
\hline & $\boldsymbol{R}_{Q}=\mathbf{1 5} \mathbf{~ k m}$ & $\boldsymbol{R}_{Q}=\mathbf{3 5} \mathbf{~ k m}$ & $\boldsymbol{W}_{Q}=\mathbf{5} \mathbf{~ k m}$ & $\boldsymbol{W}_{\mathbf{Q}}=\mathbf{2 0} \mathbf{~ k m}$ \\
\hline $\begin{array}{c}\text { Orbital speed } \\
\left(\mathbf{m s}^{-1}\right)\end{array}$ & 0.85 & 5.37 & 1.18 & 5.42 \\
\hline $\begin{array}{c}\text { Orbital radius } \\
(\mathbf{k m})\end{array}$ & 1.7 & 10.78 & 2.36 & 10.88 \\
\hline $\boldsymbol{R}_{\boldsymbol{v} v / \partial t}(\mathbf{k m})$ & 15 & 35 & 25 & 25 \\
\hline $\begin{array}{c}\partial \boldsymbol{v} / \boldsymbol{\partial t} \mathbf{r a d i a l} \\
\text { interval }(\mathbf{k m})\end{array}$ & 20 & 30 & 10 & 40 \\
\hline
\end{tabular}

\section{Model Reinitialization (Episodic Forcing)}

Mean-vortex response to episodic forcing (e.g., CGW) illustrates the importance of rotating, periodic eyewall convection in generating wavenumber-1 VRWs that cause vortex motion, and force wave transport. The BND model initially operates normally so that forced asymmetries and expected vortex behavior become well established (Fig $37 \mathrm{a}, \mathrm{c}, \mathrm{e})$. First, the forcing is turned on at the beginning of the model run, then reduced by half after 12 simulated hours, and remains fixed until the end. Despite halved forcing, the trailing spirals still symmetrizes but the forced dipole's amplitude weakens quickly compared to a normal model run (Fig 37b). Nevertheless, VRW propagation continues, as evidenced by the filaments within the outer ring of interlocked vorticity spirals. 
Trochoidal track plot orbits are temporarily disrupted before recovering, at a smaller radius $(3.7 \mathrm{~km})$. Mean orbital speed also abruptly changes at 12 hrs from 2.4 to $1.3 \mathrm{~ms}^{-1}$ (Fig 38a,b). Nonetheless, the linear system enables the vortex center to still complete the same number of orbits as the forcing orbital period.

The second experiment reduces the forcing amplitude to zero $12 \mathrm{hrs}$ into the simulation, which stops VRW excitation. Unsurprisingly, the vortex responds strongly. By 24 hrs, the forced $\zeta$ dipole is gone, but numerous residual vorticity filaments and tightly wound spirals remain near the critical radius (Fig 37d). The initially trochoidal track slows to a gradual leftward bend at time of reinitialization, and overall motion stops by the end of the simulation (Fig 38c,d).

Amplifying the forcing concludes the reinitialization experiments. Here, the forcing amplitude at 12 simulated hours. The amplitude of $\zeta$ increases quickly. Trailing spirals wind tightly around the critical radius (Fig 37f). The mean orbital speed jumps to nearly $5 \mathrm{~ms}^{-1}$, and the orbital radius doubles (Fig 38e,f). When the model reinitializes, $\omega$ remains unchanged, so that a faster-moving vortex must have larger orbits. Reinitializing the model represents the last set of sensitivity studies involving the adjustment of initial forcing parameters. The final sensitivity study here runs the model on a $\beta$-plane. 

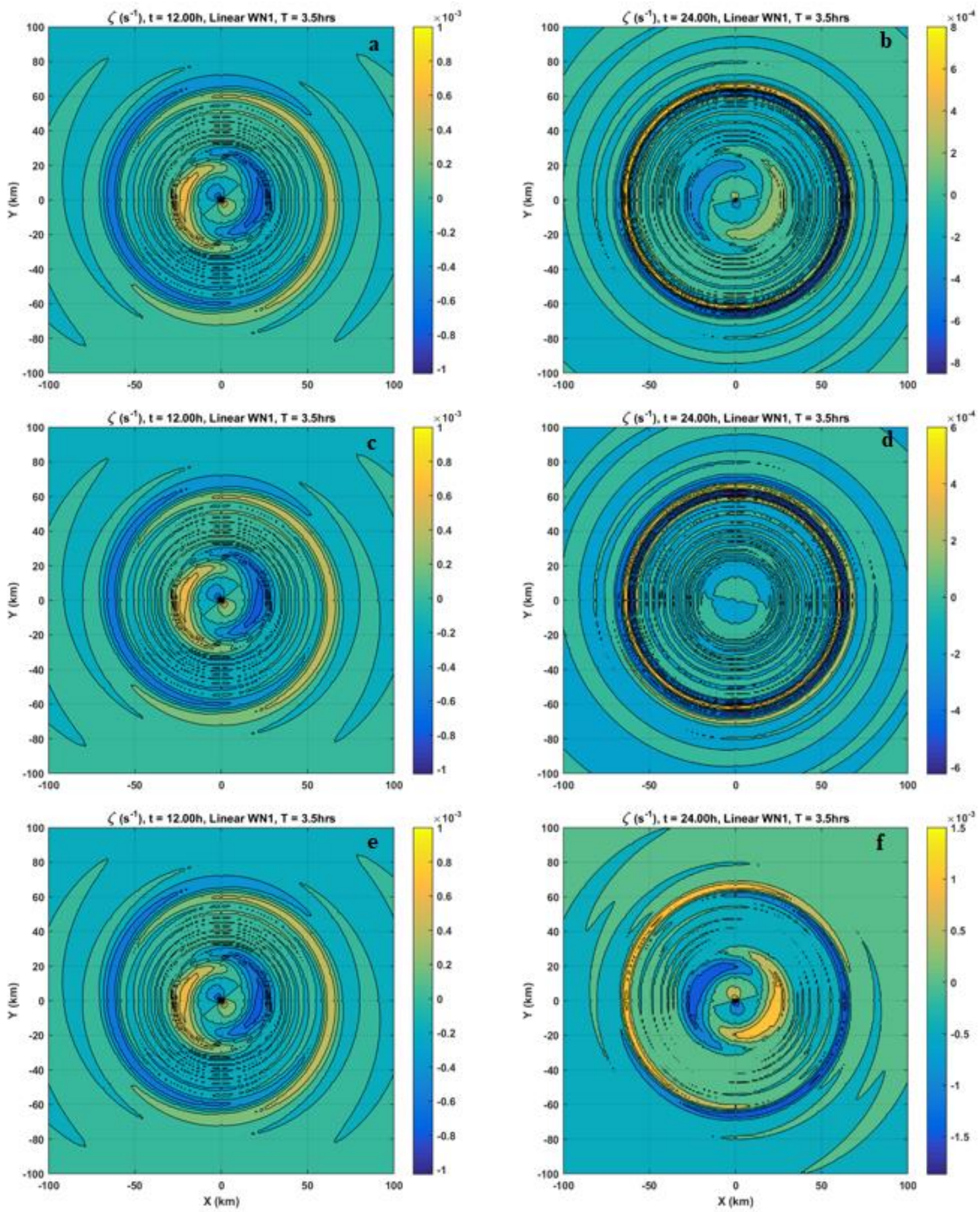

Fig 37. Vorticity contour plots before (left column) and after model reinitialization (right column) for when the forcing amplitude is halved (b), reduced to zero (d), and doubled (f). 

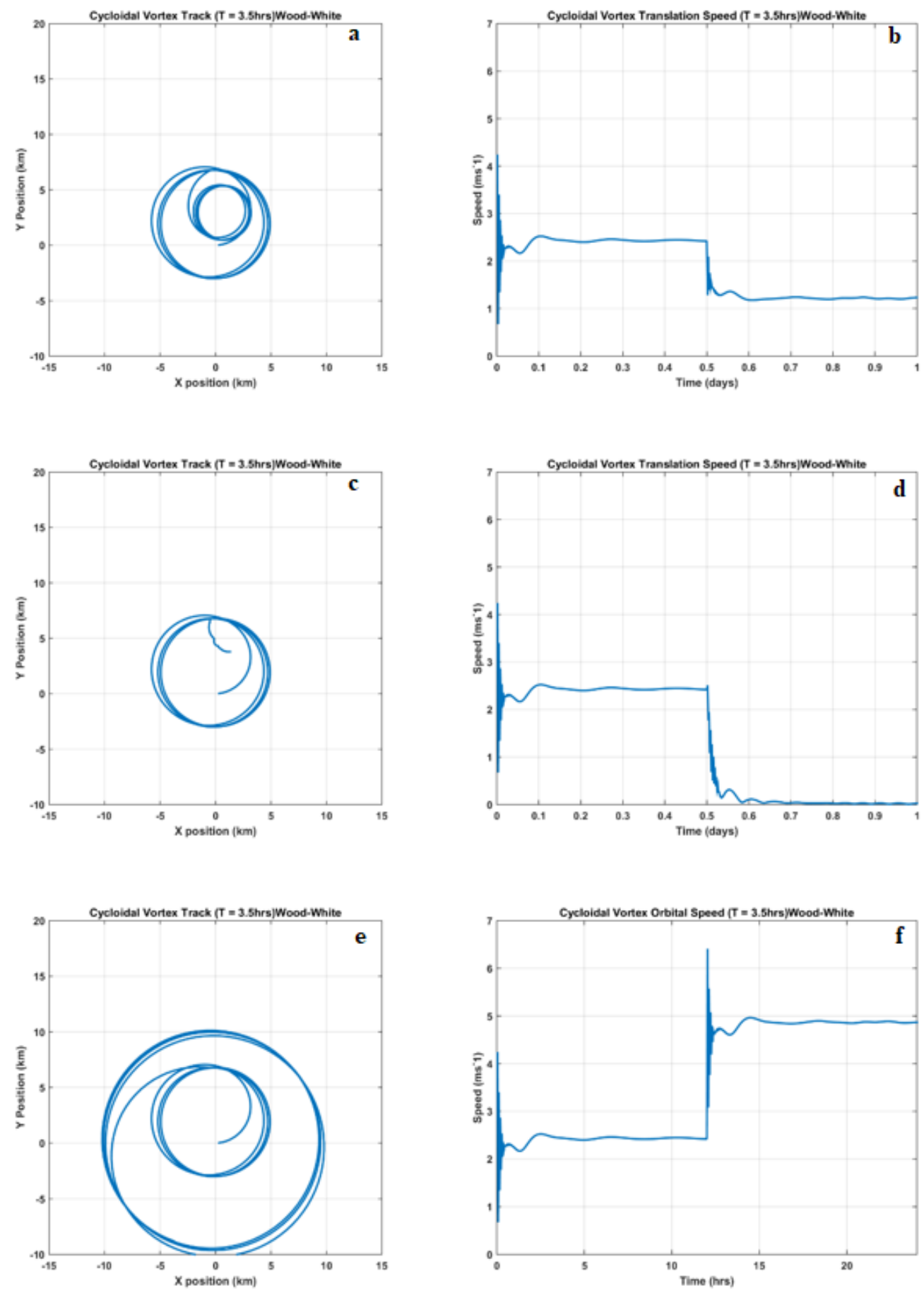

Fig 38. Vortex track (left column) and orbital speed (right column) when forcing amplitude is halved $(a, b)$, reduced to zero $(c, d)$, and doubled $(e, f)$. 
Beta-Plane Simulations

General Overview

The BND model has been run exclusively on an $f$-plane to isolate the effects of eyewall forcing on VRW propagation, vortex motion and structure, and eddy flux budgets. Large-scale, meteorological phenomena, however, are affected by $\beta$, the meridional gradient of $f$. Recall that the mean, TC swirling flow advects air around the vortex such that $\zeta$ decreases ( $f$ increases) to north and increases ( $f$ decreases) to the south so that absolute vorticity is conserved. Beta gyres consequently form at the vortex periphery and advect axially symmetric, mean vorticity across the center to cause the classic northwestward $\beta$-drift.

Vortex motion on a $\beta$-plane using both linear and nonlinear, barotropic nondivergent model, was the focus of GCW. Linear wave-mean flow interactions produced wavenumber-1 $\beta$-gyres that accelerated the mean vortex northwestward at speeds approximately four times faster than the observed $1-2 \mathrm{~ms}^{-1} \beta$-drift. In the nonlinear model, wave-wave interactions forced wavenumber-2 solutions which interacted with linear, wavenumber 1 to yield oppositely oriented, wavenumber- 1 "anti- $\beta$ gyres" (Fig 39). The anti- $\beta$-gyres counteracted the ventilation flow of the linear solution to reduce the vortex's translation speed to reasonable values. 

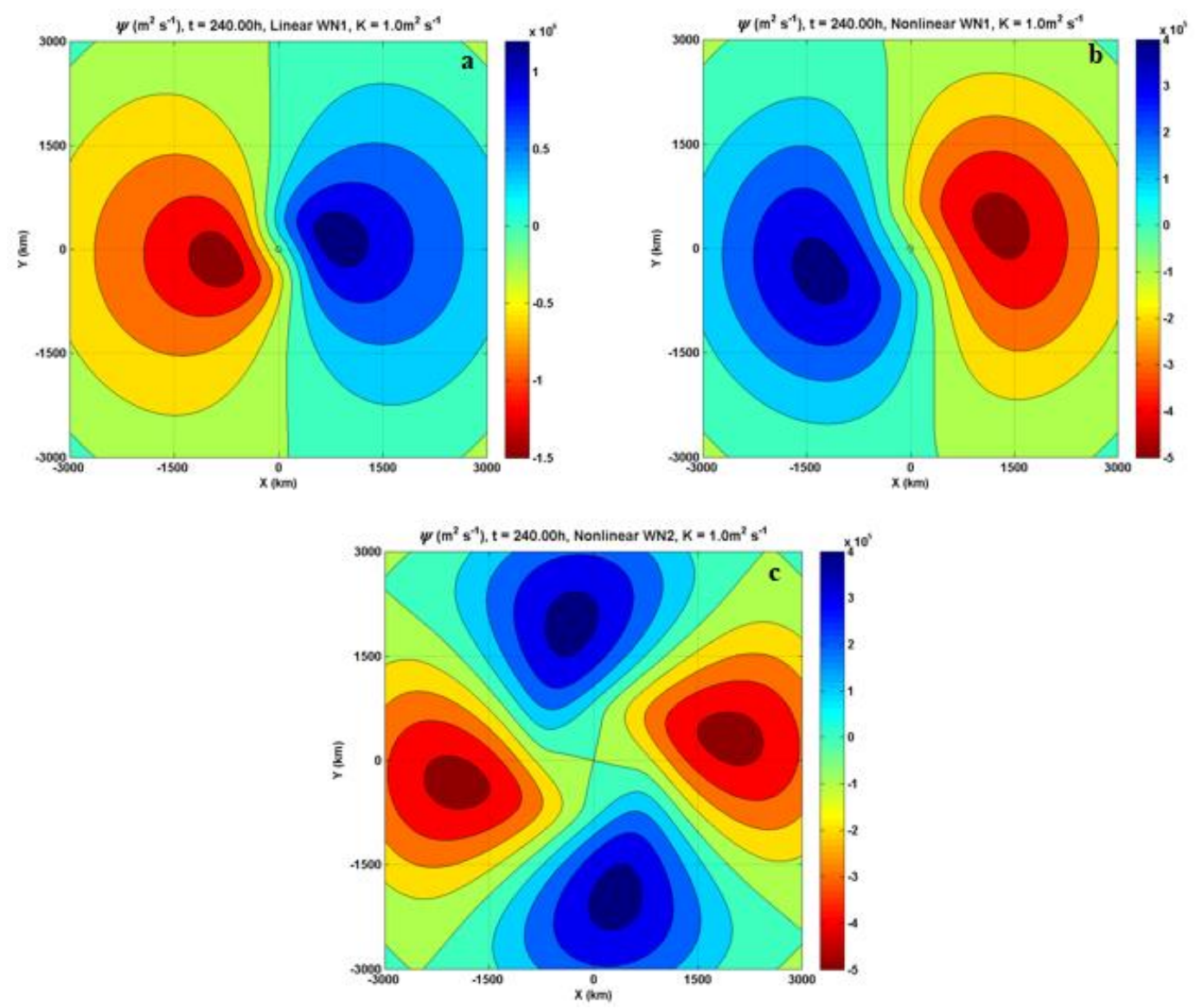

Fig 39. Complete nonlinear solution for streamfunction from GCW's barotropic nondivergent model on a beta plane illustrating the linearly forced wavenumber-1 beta gyres (a), nonlinearly forced wavenumber-1 anti-beta gyres (b), and nonlinearly forced wavenumber-2 gyres (c).

Although GCW provided a nice synthesis on VRW-induced vortex motion on a $\beta$ plane, rotating mass source-sink pairs were not incorporated to simulate small-scale motion. Therefore, the final sets of sensitivity studies performs simulations on a $\beta$-plane with an imposed rotating mass source-sink pair in the eyewall. Beta-plane simulations first consider an alternative form of the radial momentum equation (6) that contains a $\beta$ forcing term: 


$$
\frac{\partial u}{\partial t}+\frac{V}{r} \frac{\partial u}{\partial \lambda}-\xi_{0}\left(v-c_{\lambda}\right)+\frac{\partial \phi}{\partial r}=\beta r V \cos \lambda+F_{r}
$$

The first term on the right-hand side of the equal sign in (25) is the $\beta$-forcing, where $\beta=$ $\left(4 \pi \mathrm{J}^{-1} \cos \pi \varphi\right) / R_{E}$, consistent with GCW; $\mathrm{J}$ is one Julian Day $(86146 \mathrm{~s}), \varphi$ is fixed at $20^{0} \mathrm{~N}$ latitude, and $R_{E}$ is the radius of the Earth $(6371 \mathrm{~km})$. Taking $r^{-1} \partial / \partial \lambda$ of $(25)$ and $\partial / \partial r+$ $1 / r$ of (7), followed by subtraction eliminates the geopotential terms and yields the forced nondivergent vorticity equation on a $\beta$-plane:

$$
\frac{\partial \zeta}{\partial t}+\frac{V}{r} \frac{\partial \zeta}{\partial \lambda}+\left(u-c_{r}\right) \frac{\partial \zeta}{\partial r}=-\beta V \sin \lambda+Q
$$

As before, streamfunction is obtained by taking the Poisson inversion of vorticity. Exponential solutions are then assumed in frequency in azimuth, from which the dispersion relation can be derived. The sine function connected to the $\beta$-forcing is the imaginary part of the solution (hereafter $Q_{B}$ ). In (26), $Q$ represents the rotating mass source-sink pair imposed at the RMW (see equation 10), with a fixed 3.5-hr orbital period. Going forward, the source-sink pair will be denoted as $Q_{A}$ to be distinct from $Q_{B}$. It is important to note however, that the total response is a superposition of two linear solutions that are purely additive with no interaction. Since $Q_{B}$ has whole-vortex spatial scale results, the $\beta$-plane solution will have horizontal dimensions $\sim 2500 \mathrm{~km}$. Beta-plane simulations are categorized into "normal model run", and "reinialized runs". The latter involves reducing the forcing amplitude to zero, halfway into the 24 -hr simulation. 
Reinitialization is important to understanding the individual contributions of each forcing to vortex motion.

\section{Normal Model Run}

The total forcing produces large, trailing vorticity spirals of opposite polarity that extend outward to $2000 \mathrm{~km}$ and have maximum amplitude centered approximately 300 $\mathrm{km}$ from the vortex center. The spirals wrap tightly around a large, symmetric area of highly filamented vorticity. The eye-like feature at the center is the inner waveguide where VRWs, forced by $Q_{A}$, propagate outward to the critical radius (Fig 40a). Vortexscale $\psi$ shows forced $\beta$-gyres as a large wavenumber- 1 dipole centered radially at $\sim 1200$ $\mathrm{km}$ from the vortex center on each side, and extending outward to $>2500 \mathrm{~km}$ (Fig 40b). The gyres produce a counterflow across the center that advects axially symmetric, mean vorticity toward the northwest. Overall the total forcing causes a large-scale, northwestward track with small-scale trochoidal oscillation that become less pronounced with time (Fig 40c) and closely resembles observed trochoidal tracks (e.g., Fig 6). The vortex travels $\sim 153 \mathrm{~km}$ over $24 \mathrm{hrs}$, at an average speed of nearly $2 \mathrm{~ms}^{-1}$. However, translation speed consists of growing high-frequency/high-amplitude oscillations forced by $Q_{A}$ (Fig 40d). The vortex completes nearly 7 complete orbits during the simulation (Fig 40d). 

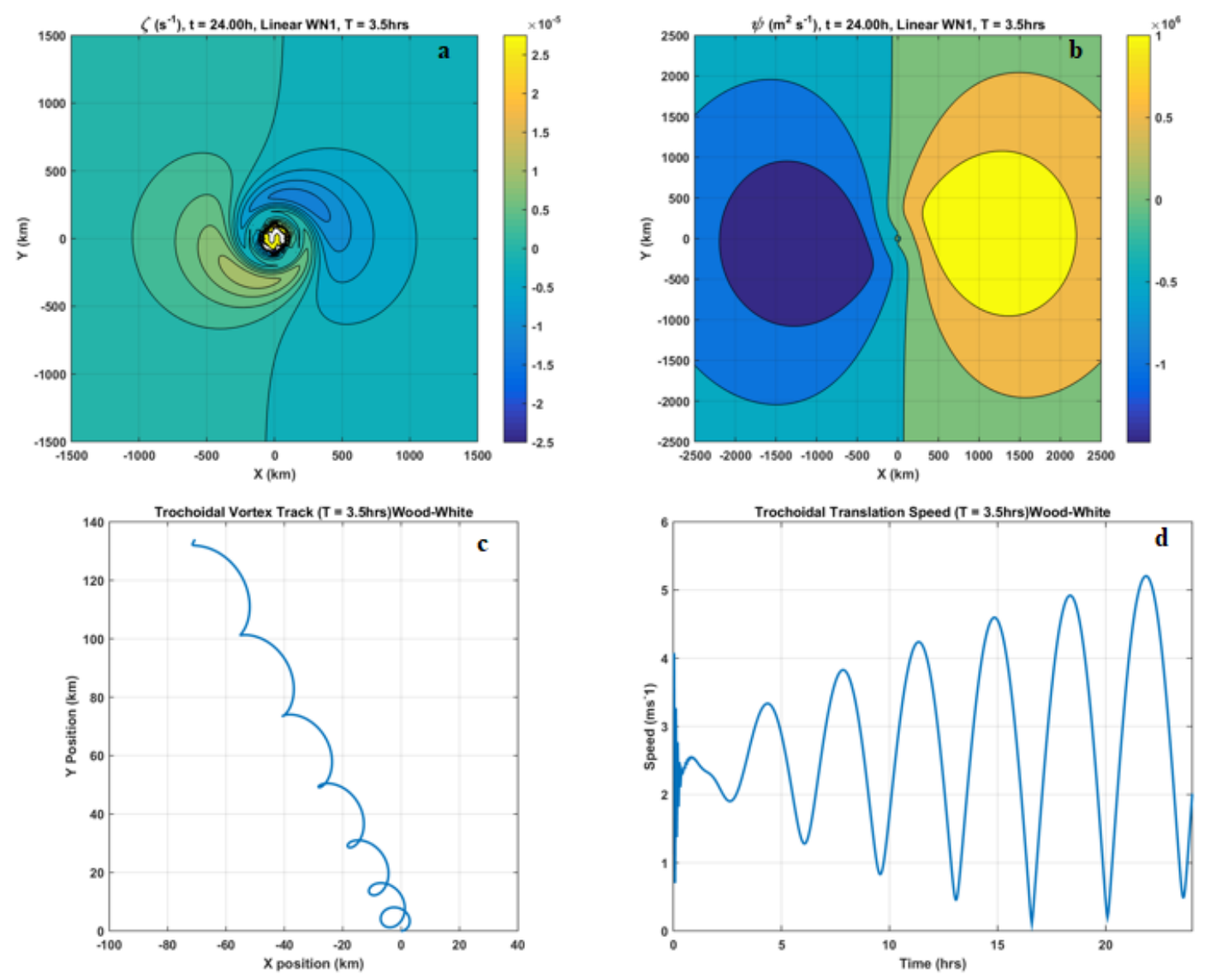

Fig 40. Beta-gyre vorticity (a) and streamfunction (b) dipole, with induced trochoidal track (c) and translation speed (d).

\section{The Outer Waveguide}

Since the $\beta$-plane simulation has a whole-vortex scale in an asymptotically bounded vortex, there exists an outer waveguide that is an important consideration for vortex motion. The mean vortex's circulation approaches zero at large radius. Inside the RMW, the flow is solid rotation. Immediately outside the RMW, the mean wind and the vorticity decreases with radius. The latter reverses sign at $\sim 250 \mathrm{~km}$, becomes anticyclonic, and then asymptotes to zero (Fig 41a). The radial vorticity gradient $(\partial \zeta / \partial r)$ also reverses sign at $\sim 400 \mathrm{~km}$. The narrow radial interval where $\partial \zeta / \partial r$ is weakly positive 
constitutes the outer waveguide - bounded by an inner critical radius and outer turning point (Fig 41b). Therefore, free waves corresponding to the $\beta$-gyres would propagate slowly with a positive (downstream) phase velocity and negative (upstream) group velocity. Beta-forced VRWs would propagate freely with $\Omega_{1 \mathrm{D}}>\Omega>0$, and be evanescent when $\Omega<0$ or $\Omega>\Omega_{1 \mathrm{D}}>0$, the reverse scenario of the inner waveguide.

Inward-propagating waves are Doppler-shifted to zero frequency and absorbed at the critical radius while outward-propagating waves are Doppler-shifted to $\Omega_{1 \mathrm{D}}$, reflect from the turning point, and ultimately propagate inward to the critical radius to become highly filamented and absorbed. Filamentation is modulated by second-order diffusion and acts as the primary mechanism that limits the linear $\beta$-drift acceleration that was once thought (W92) to be driven by the excitation of a normal mode. Fourth-order diffusion (e.g., Montgomery et al. 1999) by contrast, dampens linear vortex motion by "eating up" vorticity filaments more rapidly at the critical radius, as described by GCW. Furthermore, primitive equation model of W92 that used velocity potential as the prognostic variable, instead of vorticity, were much less dissipative, which consequently produced unbounded, linear vortex acceleration. 

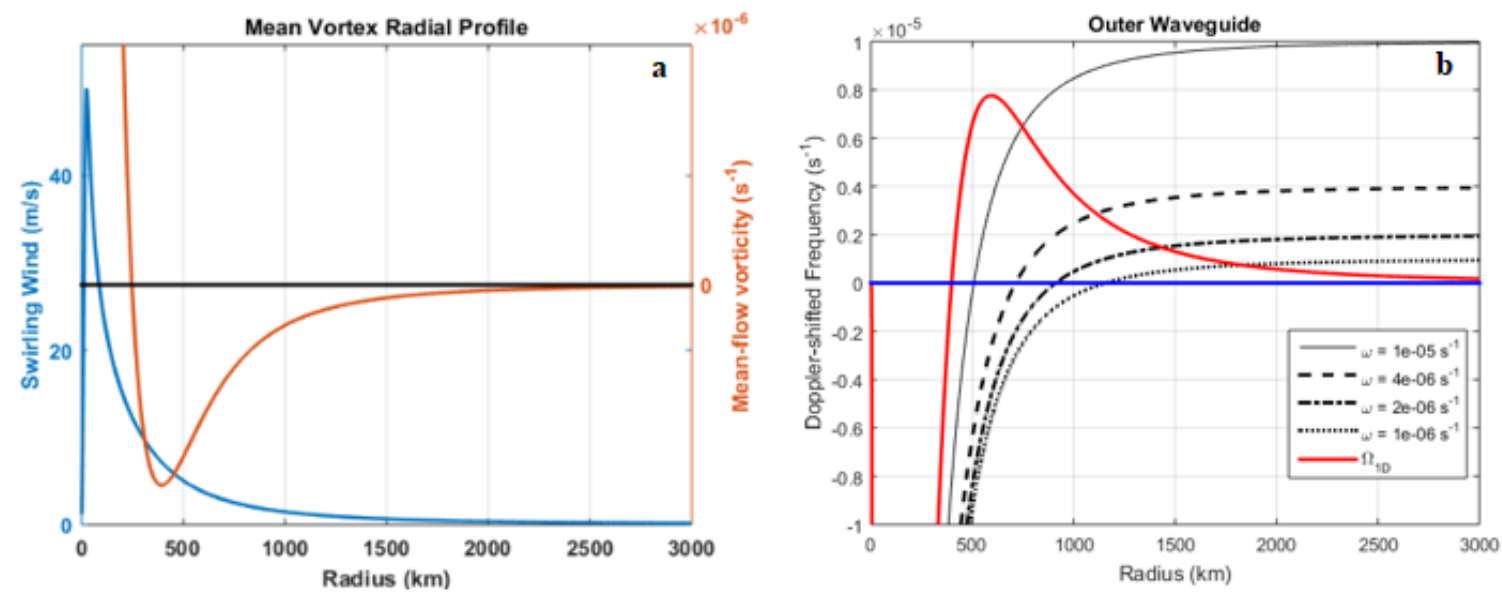

Fig 41. Mean-vortex radial profile extending out to $3000 \mathrm{~km}$, showing reversal of meanflow relative vorticity, becoming anticyclonic on the periphery before asymptoting to zero (a); corresponding outer waveguide supporting propagation of only very low-frequency waves upon the weakly positive radial vorticity gradient (b).

\section{Reinitialization}

To further understand the total forcing's effect on vortex motion on a $\beta$-plane, the model is reinitialized halfway through the simulation. Recall that reinitializing the convectively forced model on an $f$-plane with zero forcing causes the convectively forced gyres to decay quickly, which rapidly slows vortex motion. If one transitions from a $\beta$ plane to an $f$-plane at the model run's midpoint, then the $\beta$-gyres persist for the remainder of the simulation without any major structural changes, consistent with Willoughby (1988), W92, and GCW (Fig 42b). However the main difference with the preceding studies is that the present reinitialized model run includes an active rotating mass sourcesink pair.

The $\beta$-gyres' persistence prompts the vortex to continue moving northwestward despite the $f$-plane transition; although the track is not as large $(\sim 103 \mathrm{~km})$. Another notable change is that the trochoidal component becomes more prominent because $Q_{A}$ is 
unaffected by this reinitialization. Mean translation speed $\left(\sim 1.19 \mathrm{~ms}^{-1}\right)$ remains oscillatory throughout the simulation, reaching a maximum at the reinitialization point before a decreasing (Fig 43a,b). Based on the results, it is clear that the $\beta$-gyres continue to influence the large-scale northwest drift despite zero forcing. Whole-vortex spatial scale explains the $\beta$-gyres' persistence for long times, in contrast with the short-lived mass source-sink solution.

If $Q_{A}$ is instead reduced to zero on a $\beta$-plane, the rotating mass source-sink pair disappears while the $\beta$-gyres remain unaffected. The total vortex motion consists of an initially wobbly NW drift that becomes smoother after reinitialization and travels $\sim 157$ $\mathrm{km}$. Translation speed $\left(\sim 1.8 \mathrm{~ms}^{-1}\right)$ changes from highly oscillatory to a more linear increase with some residual oscillations (Fig 43c,d). The ripples' presence are likely attributed to a slowly growing barotropic instability. Nevertheless, the results suggest that $Q_{A}$ 's influence on the track oscillations slightly decreases the vortex's overall translation speed on a $\beta$-plane. Once $Q_{A}$ is removed, the $\beta$-gyres control the vortex track. In order to fully synthesize the total forcing on a $\beta$-plane however, the final reinitialization simultaneously turns off both $Q_{A}$ and $Q_{B}$.

When the total forcing is completely shut off halfway through a $\beta$-plane simulation, the initial wobbly northwest drift quickly becomes a more linear track. The vortex travels $\sim 109 \mathrm{~km}$, slightly faster than the first reinitialization run. Translation speed $\left(1.26 \mathrm{~ms}^{-1}\right)$ changes from highly oscillatory to a slowing linear acceleration (Fig 43e,f). Nevertheless, the $\beta$-gyres' persistence with zero forcing is still apparent, so that the vortex continues northwestward motion, albeit slowly. A common result is that from all four $\beta$-plane simulations' speeds stay within a 1-2 $\mathrm{ms}^{-1}$ range (summarized in Table 
4). Previous work (e.g., Willoughby 1988, Willoughby 1992, Montgomery et al. 1999, Gonzalez et al. 2015) showed that only nonlinear or linear models with strong diffusion yielded comparable $\beta$-drift accelerations. Despite the intriguing results, nonlinear interactions are the mechanism that limits northwestward $\beta$-plane motion in nature
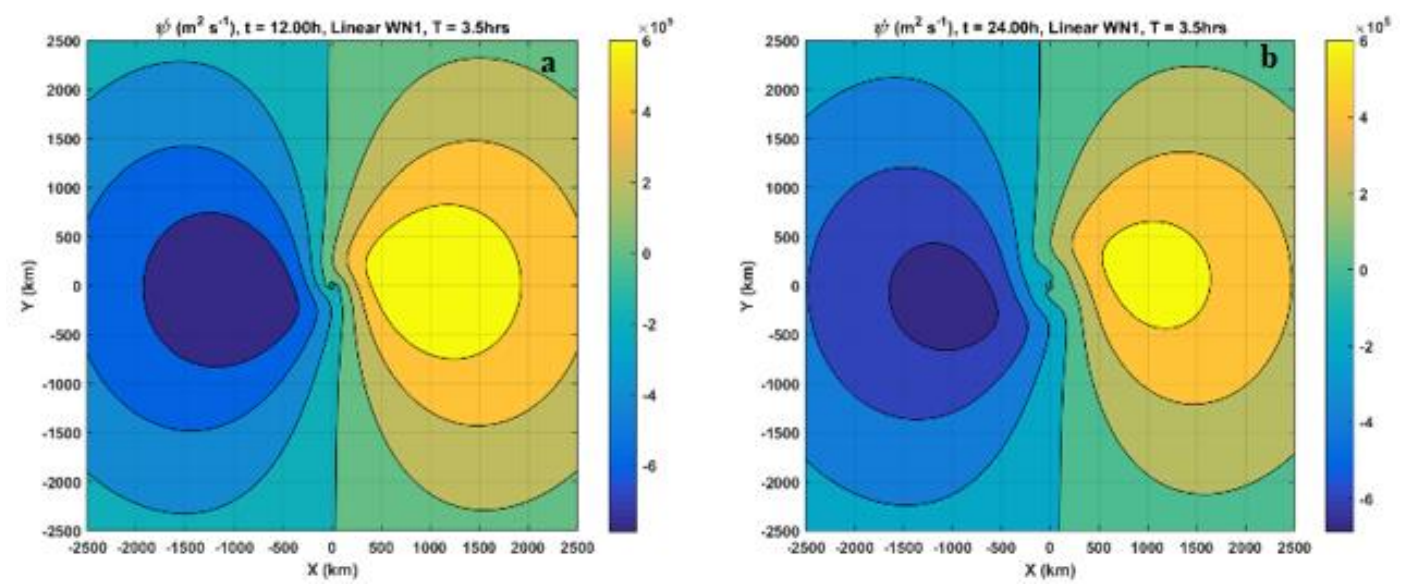

Fig 42. Beta-gyre streamfunction dipole before (a) and after (b) reinitialization. 

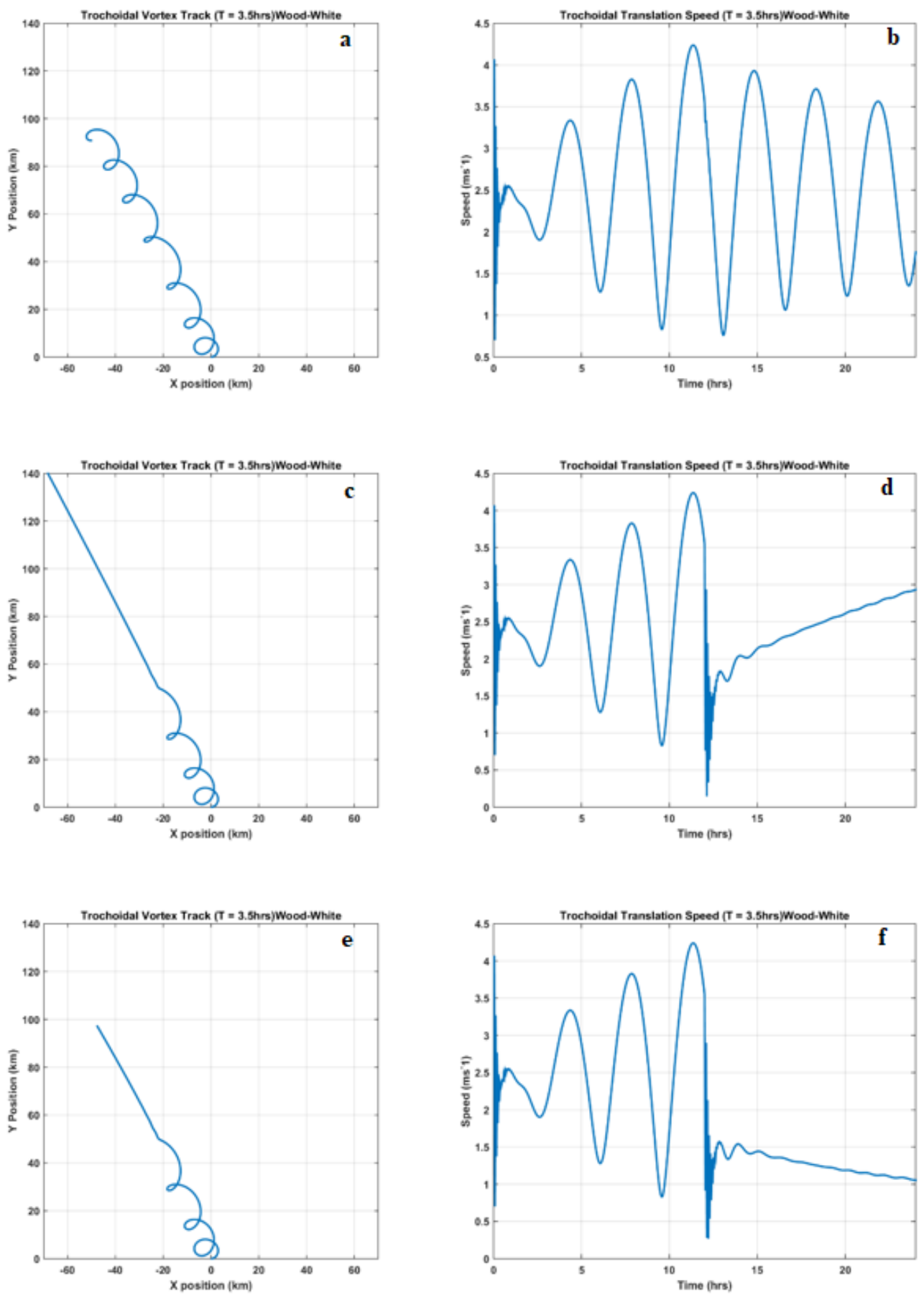

Fig 43. Vortex track (left column) and translation speed (right column) for zero beta $(\mathrm{a}, \mathrm{b})$, zero convective forcing (c,d), and total forcing reduced to zero (e,f). 
Table 4. Vortex motion results from normal beta-plane simulations versus reinitialized model runs.

\begin{tabular}{|c|c|c|c|c|}
\hline & Normal run & $\boldsymbol{Q}_{A} \neq \mathbf{0} ; \boldsymbol{Q}_{\boldsymbol{B}}=\mathbf{0}$ & $\boldsymbol{Q}_{\boldsymbol{A}}=\mathbf{0} ; \boldsymbol{Q}_{\boldsymbol{B}} \neq \mathbf{0}$ & $\begin{array}{c}\text { Total forcing }= \\
\mathbf{0}\end{array}$ \\
\hline $\begin{array}{c}\text { Distance } \\
\text { traveled (km) }\end{array}$ & 153 & 103 & 157 & 109 \\
\hline $\begin{array}{c}\text { Mean } \boldsymbol{\beta} \text {-drift } \\
\text { speed through } \\
\mathbf{2 4} \text { hrs }\left(\mathbf{m s}^{-1} \text { ) }\right.\end{array}$ & 1.77 & 1.19 & 1.81 & 1.26 \\
\hline
\end{tabular}

Summary

Varying the model's forcing parameters offers additional insights into wavenumber-1 vortex Rossby waves. Imposing the "convective" forcing near, but not directly at the radius of maximum wind causes the waves to accelerate the mean flow at the displaced radii. Mean-flow acceleration inside the eye leads to essentially no net intensification at the radius of maximum wind, and a deceleration in the neighborhood of the critical radius. The latter coincides with strong angular momentum flux divergence. What are then, the intensity change implications for a vortex whose mean flow is accelerated at different radii when the forcing radius changes? Vortex motion and meanflow acceleration are most sensitive to the forcing's radial width. Narrow forcing produces slow orbital speed and small orbital radii. Radial extent of the mean-flow acceleration in the core is strongly controlled by forcing width. A wide forcing accelerates the mean flow over a larger area with a stronger peak at the forcing radius and faster vortex trochoidal motion. 
Model reinitialization shows how episodic forcing affects vortex Rossby wave excitation and vortex motion. The model runs normally for the first 12 hors of the simulation and then the forcing amplitude is reduced by half for the rest of the run. Convectively forced gyres weaken but persist. Reinitialization temporarily disrupts the trochoidal track before the vortex recovers to meander cyclonically with a smaller orbital radius and slower orbital speed. Reducing the forcing amplitude to zero halfway through the simulation yields a more dramatic vortex response. Convectively forced gyres disappear, but numerous vorticity filaments, and interlocked vorticity spirals near the critical radius persist to the end of the simulation. The streamfunction shows a weak distorted flow on opposite sides of residual ring of filaments near the critical radius. Vortex motion degenerates into a slow leftward bend and ultimately stops. If the forcing amplitude is instead doubled, then the trochoidal orbits become larger, and orbital speed increases.

Running the convectively forced model on a beta plane provides a more complicated vortex motion through a linear superposition of a rotating mass source-sink pair and beta forcing. Vorticity and streamfunction are organized into inner gyres forced by the mass source-sink, and outer beta gyres forced by, planetary vorticity advection. The combined gyres produce a large-scale northwest track, with small-scale wobbles superimposed. The beta gyres are vortex Rossby waves in the outer waveguide where the mean radial vorticity gradient is weakly positive. Only low-frequency, cyclonicpropagating free waves can exist in the outer waveguide. Inward-propagating waves are Doppler-shifted to zero frequency and absorbed at the critical radius. Outwardpropagating waves are Doppler-shifted to the cutoff frequency and reflected from the 
turning point before ultimately reaching the inner critical radius where the energy is absorbed. By contrast, frequency passbands in the inner waveguide are confined by an inner turning point and outer critical radius. The locus of vorticity gradient sign reversal acts as the boundary between the waveguides, with each containing vortex Rossby waves propagating with different frequencies and oppositely directed tangential group and phase velocities.

Three beta-plane reinitialization runs conclude the forcing sensitivity studies. The first reduces beta to zero halfway through the model simulation while the mass sourcesink pair remains unaffected so that the mean vortex transitions to an $f$-plane. The beta gyres persist because energy loss at the outer waveguide's critical radius is slow. The translation remains oscillatory, but reaches a peak amplitude at the beta-to-f-plane transition and then slows. In the second reinitialization run, the mass source-sink pair stops halfway through the simulation without turning off the beta effect. The immediate response is an abrupt transition from a wobbling northwestward track to a smooth motion in the same direction, during which the mean translation speed increases. The final reinitialization run turns off the total forcing and causes the initially wobbly vortex to assume a smooth beta-drift motion with the highly oscillatory speed transitioning to a slowing linear acceleration.

Beta-plane reinialization highlights each forcing's contributions to vortex motion. The mass source-sink pair is responsible for small-scale trochoidal oscillations. The beta gyres are responsible for the large-scale northwestward track, with the persistent asymmetries that maintain the track for a long time after the beta-forcing is reduced to zero. These conclusions arise from a superposition of two linear solutions. The mass 
source-sink pair and beta forcing do not interact with each other. An interaction could only occur in a nonlinear framework. Nevertheless, beta-plane simulations help in understanding each gyres' inherent time scales and contribution to vortex motion on both an $f$-plane and beta-plane, and highlights the distinctions of the inner and outer waveguide. This dissertation now shifts to key mean-vortex parameters in Chapter 5. Circulation dynamics are also applied to vortices to help evaluate the consistency of vorticity monopoles on a spherical Earth. 


\section{CHAPTER V. VORTEX SENSITIVITY STUDIES AND WIND-PROFILE}

\section{ANALYSES}

\section{General Overview}

Chapter 4 first showed that forcing radius and width controlled locus and radial extent of the mean-flow acceleration. Then model reinitialization explored episodic forcing where shutting off the forcing halfway through the simulation, caused wave and trochoidal vortex motion to decay quickly, whereas amplified forcing sped up the orbital track. Additionally, combined simulations explored differing timescales through superposition of a convective and beta-effect forcing. The two linear solutions yielded more complete vortex motion with a large-scale northwestward beta drift combined with small-scale, convectively induced trochoidal wobbles. Beta gyres arising from planetary vorticity advection was responsible for the northwestward drift, and consist of vortex Rossby waves propagating within a narrow outer waveguide. Lastly, beta-plane reinitalization showed that unlike the convectively forced gyres, the beta gyres persisted for a long time after the forcing stopped so that the northwestward track continued, albeit at a reduced speed, with residual low-amplitude oscillations.

Chapter 5 further explores sensitivity by first focusing on changes in the mean vortex's initial intensity to evaluate the influences on VRW propagation and vortex motion. The sensitivity model runs consider vortices at tropical depression (TD), strong tropical storm (TS), and category-5 (CAT5) intensity. The maximum wind $\left(V_{m}\right), \mathrm{RMW}$, and the outer power-law exponent $\left(N_{\text {out }}\right)$ are altered simultaneously to best reflect TC 
climatology. Wind-profile shape narrows the waveguide; as wind profile becomes sharper. In addition, the forcing is imposed at the adjusted RMW, and the amplitude is slightly reduced for weak vortices and conversely. Constraints on parametric TC wind profiles in terms of the Circulation (Stokes') Theorem, are also explored to avoid logical contradictions implicit in "unbounded vortices". Lastly, two types of "finitely bounded" vortices and an unbounded vortex are implemented to understand how changes to the wind profiles' asymptotic properties affect the inner waveguide. For convenience, only excited waves propagating with $\omega=0.25 \omega_{\mathrm{o}}$ are explored.

\section{Vortex Initial Intensity}

Results

Tropical cyclone intensity is usually measured in terms of $V_{m}$ in the eyewall. Radius of maximum wind is correlated with TC strength, since strong storms generally have smaller eyes. For example, intensity fluctuations occur during eyewall replacement cycles in which outer eyewalls become dominant at larger radius with weaker winds (Willoughby et al. 1982). Since many other TC properties tend to scale with a changing RMW, $V_{m}$ and $N_{\text {out }}$ are also altered simultaneously so that the combined eye size, wind speed, and shape of the outer wind profile best match the intensity for TD, TS, and CAT5 hurricanes.

In the TD-simulation, $V_{m}$ is initialized at $15 \mathrm{~ms}^{-1}$ with a $75-\mathrm{km} \mathrm{RMW}\left(\omega_{\mathrm{o}}=\right.$ $2 \times 10^{-4} \mathrm{~s}^{-1}$, and $N_{\text {out }}=-0.25$. These values produce a broad, but weak wind field, with a fairly flat profile and a wide inner waveguide (Fig 44a,c). In addition, the forcing 
amplitude is reduced by $75 \%$ of the default value $\left(1 \times 10^{-6}\right)$ to reflect weaker convection observed in tropical depressions. Excited waves with an orbital period of $35 \mathrm{hrs}$ $\left(2 \pi / 0.25 \omega_{0}\right)$, propagate very large distances between a $40-\mathrm{km}$ turning radius and a $190-$ $\mathrm{km}$ critical radius. The forcing dipole consists of elongated asymmetries that stretch around the RMW. A swath of weak, anticyclonic vorticity wraps around the TD-vortex's inner core, with the easternmost portion extending to the critical radius. Surrounding the forced dipole is a small vorticity ring whose radius seems to coincide with the turning point as opposed to the critical radius (Fig 44c,d). The lack of apparent vorticity filaments shows that radial VRW propagation is limited and the waves have small amplitude. Moreover, the vortex assumes a broad, cyclonic loop that almost completes a single orbit with a mean orbital speed of $7.3 \mathrm{~ms}^{-1}$ (Fig 44e,f). 

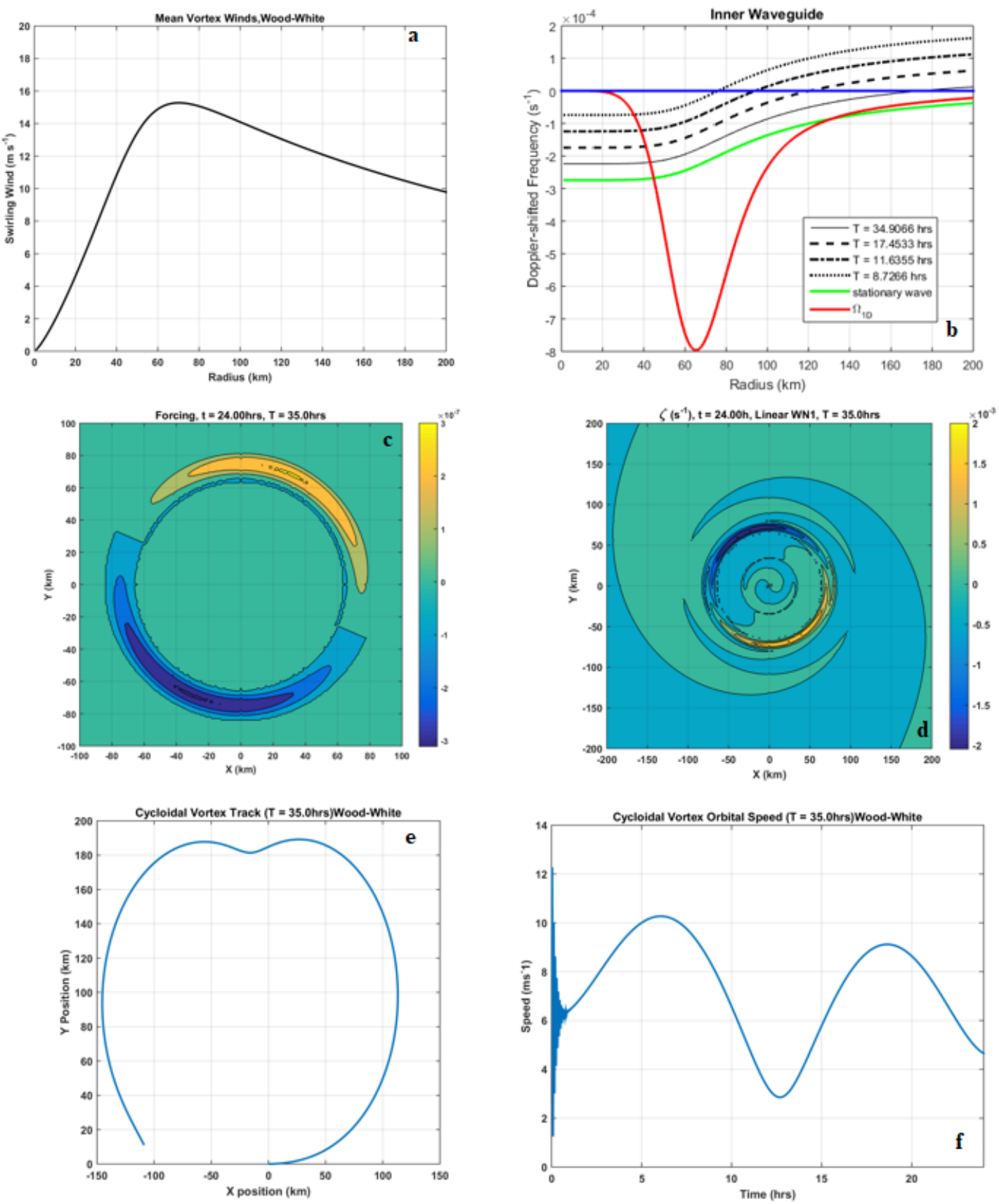

Fig 44. TD-vortex radial profiles (top row), forced wavenumber-1 dipole (mid row), and vortex motion (bottom row). 
The next simulation models a tropical storm with the following parameters: $V_{m}=$ $30 \mathrm{~ms}^{-1}, \mathrm{RMW}=50 \mathrm{~km}\left(\omega_{\mathrm{o}}=6 \times 10^{-4} \mathrm{~s}^{-1}\right)$, and $N_{\text {out }}=-0.4$. Since the winds are still relatively weak, the angular velocity at the RMW is slow, which produces a slightly narrower waveguide such that waves with the selected orbital period ( $T=11.6 \mathrm{hrs})$ propagate from $\sim 35$ and $135 \mathrm{~km}$ radius (Fig 45a,b). Asymmetries that arise from a slightly stronger forcing are less elongated and rotate faster than in the TD case (Fig $45 \mathrm{c}, \mathrm{d})$. Vortex Rossby wave propagation is more apparent with vorticity filaments radiating outward from the forced dipole before being absorbed in the neighborhood of the critical radius. The TS-vortex completes just over 2 large, cyclonic orbits with 31.5$\mathrm{km}$ radius and a $4.74 \mathrm{~ms}^{-1}$ mean orbital speed (Fig 45e,f). The orbital speed has rapid start up transients that decrease in amplitude over time. As in the previous case, the TSvortex motion is fast. 

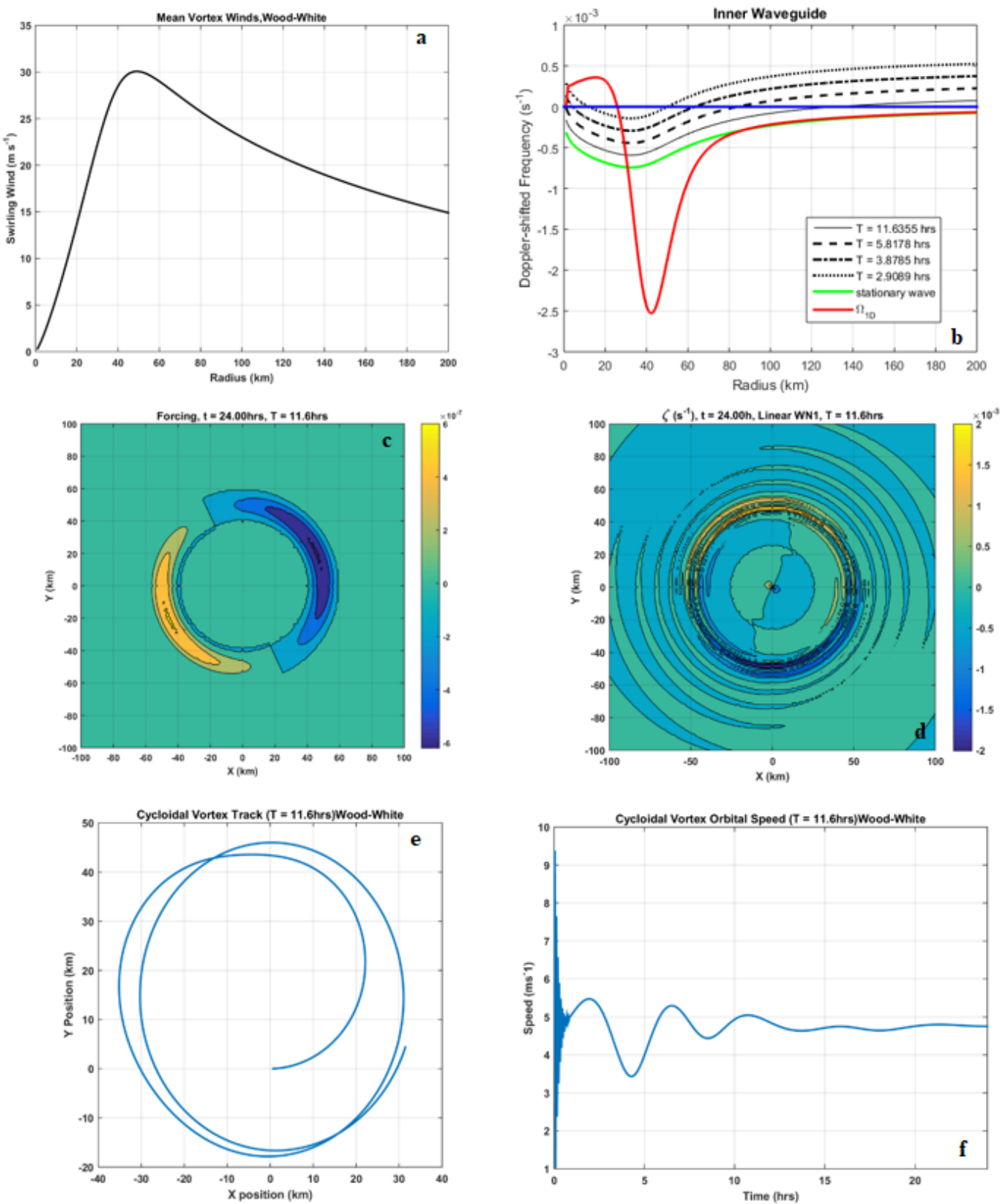

Fig 45. TS-vortex radial profiles (top row), forced wavenumber-1 dipole (mid row), and vortex motion (bottom row).

The final intensity experiment considers a CAT-5 mean vortex that contains the narrowest inner waveguide. Here, $V_{m}=70 \mathrm{~ms}^{-1}$ at $15 \mathrm{~km}\left(\omega_{\mathrm{o}}=4.67 \times 10^{-3} \mathrm{~s}^{-1}\right)$, with $N_{\text {out }}$ $=-0.75$, creating a small eye with a steep wind profile outward from the RMW (Fig 
46a,b). The forcing amplitude is increased by nearly $30 \%$ to reflect stronger eyewall convection in major hurricanes. Excited VRWs therefore must propagate with high specified frequencies. For $T=1.5 \mathrm{hrs}$, the excited waves propagate outward to the $38-\mathrm{km}$ critical radius, producing a tight ring of interlocked, vorticity filaments that surrounds the small forced dipole (Fig 46c,d). Outward from the ring is a pronounced vorticity wake that aligns with vortex motion. The vortex assumes a small-scale trochoidal track (1.25$\mathrm{km}$ orbital radius) with $\sim 16$ complete orbits, at a $1.45 \mathrm{~ms}^{-1}$ mean orbital speed (Fig $46 \mathrm{e}, \mathrm{f})$. 

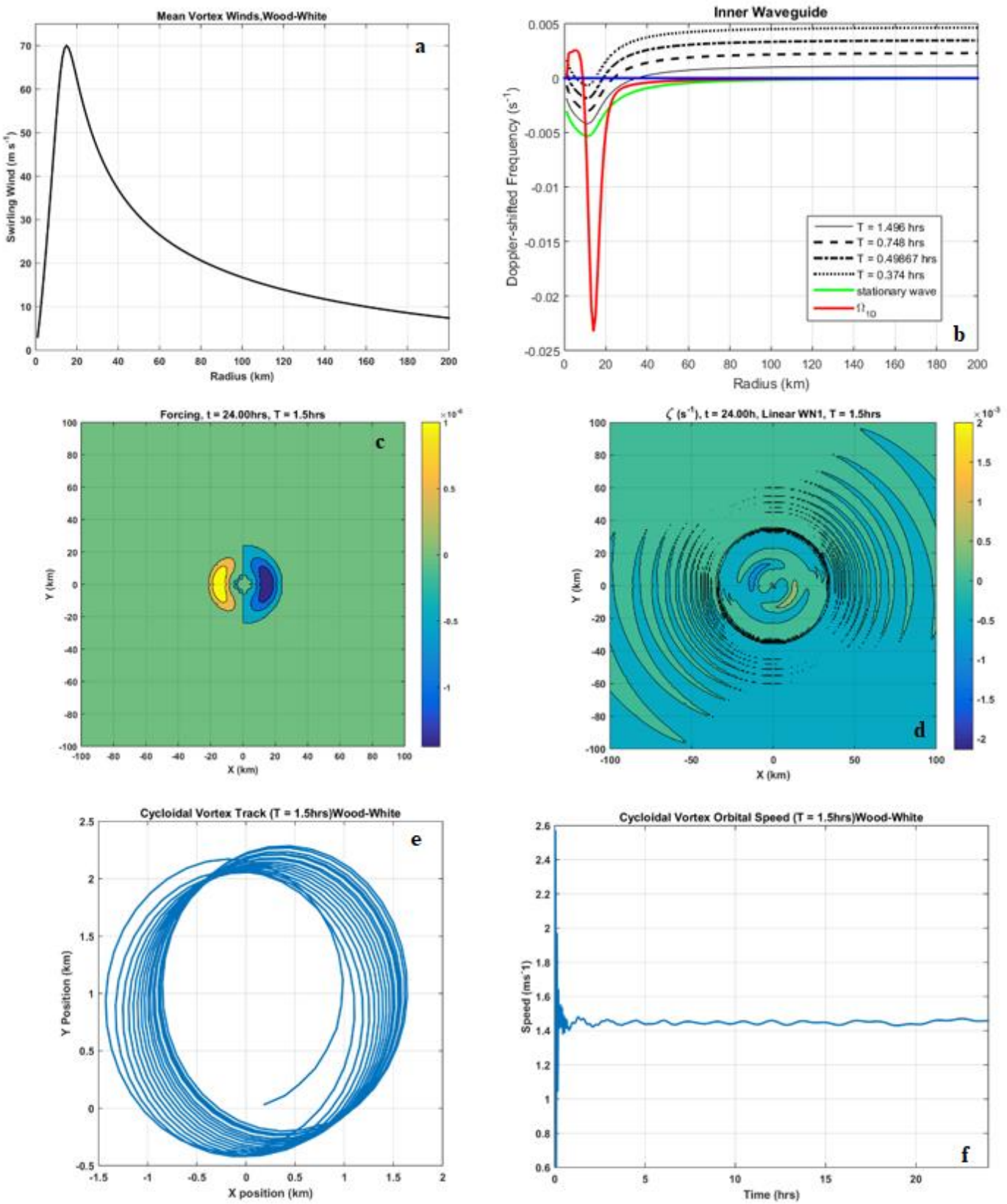

Fig 46. CAT5-vortex radial profiles (top row), forced wavenumber-1 dipole (mid row), and vortex motion (bottom row).

Climatologically, weaker $V_{m}$ coincides with larger RMW, and flatter outer-wind profiles, resulting in wider inner waveguides. However, if one were to isolate each of the three vortex parameters, then $V_{m}$, has the strongest effect on the frequency passbands' 
radial interval. The mean-flow angular velocity at the RMW $\left(\omega_{\mathrm{o}}\right)$ increases with $V_{m}$, so forced waves within intense vortices must propagate with high $\omega$ (short period) and Doppler-shift to zero at small critical radii. Conversely, larger RMW decreases $\omega_{\mathrm{o}}$, so for a large RMW, forced waves propagate with low $\omega$ (long period). Therefore, the inner waveguides of vortices with large eyes are wider. However, most TC properties scale with RMW. Lastly, $N_{\text {out }}$ has no direct effect on $\omega$, but is a strong control of the inner waveguide's width. Small $N_{\text {out }}$ results in a radially wider waveguide because the outer wind profile is flatter.

Although the model runs for TD, TS, and CAT5-vortices yield different results (summarized in Tables 5 and 6), one consistent finding is that the inner waveguide width is modulated not only by tangential wavenumber, but also by RMW and $N_{\text {out }}$. As discussed in Chapter 1, the significance of a wide inner waveguide is the increased range of frequencies with which VRWs can propagate so that the waves can transport energy and angular momentum over greater distances and form trailing spirals at larger critical radii. However, the vortex vorticity gradient must also be sufficiently strong to induce significant VRW propagation. The next sections explore how the inner waveguide geometry is affected by the circulations of bounded and unbounded vortices and addresses the physical consistency of vorticity monopoles in terms of Stokes' Theorem. 
Table 5. Vortex parameters, forcing orbital period, and waveguide width for tropical depression, tropical storm, and category-5 mean vortices.

\begin{tabular}{|c|c|c|c|c|c|}
\hline & $\boldsymbol{V}_{\boldsymbol{m}}\left(\mathbf{m s}^{-\mathbf{1}}\right)$ & $\begin{array}{c}\mathbf{R M W} \\
\mathbf{( k m})\end{array}$ & $\boldsymbol{N}_{\text {out }}$ & $\boldsymbol{T}$ (hrs) & $\begin{array}{c}\text { Waveguide } \\
\text { width (km) }\end{array}$ \\
\hline $\begin{array}{c}\text { Tropical } \\
\text { depression }\end{array}$ & 15 & 75 & -0.25 & 35 & 150 \\
\hline $\begin{array}{c}\text { Tropical } \\
\text { storm }\end{array}$ & 30 & 50 & -0.4 & 11.6 & 100 \\
\hline Category 5 & 70 & 15 & -0.75 & 1.5 & 30 \\
\hline
\end{tabular}

Table 6. Vortex motion results for tropical depression, tropical storm, and category-5 mean vortices.

\begin{tabular}{|c|c|c|c|}
\hline & $\begin{array}{c}\text { Orbital speed } \\
\left(\mathbf{m s}^{\mathbf{- 1}}\right)\end{array}$ & $\begin{array}{c}\text { Orbital radius } \\
(\mathbf{k m})\end{array}$ & $\begin{array}{c}\text { \# of complete } \\
\text { orbits }\end{array}$ \\
\hline Tropical depression & 7.27 & N/A & $<1$ \\
\hline Tropical storm & 4.74 & 31.5 & 2.07 \\
\hline Category 5 & 1.45 & 1.25 & 16 \\
\hline
\end{tabular}

Circulation and Vortical Dynamics

Stokes' Theorem Applied to Vortices

Tropical Cyclones' swirling winds are strongest in the eyewall, gradually decay outward, and smoothly blend in to the surrounding large-scale atmosphere. A vortex is said to be asymptotically bounded when the circulation asymptotes to zero at large radius (e.g., Fig 41a). If the circulation becomes identically zero at some finite radius (e.g., $1500 \mathrm{~km}$ ) the vortex is described as finitely bounded (Fig 47a,b). Both types of bounded vortices contain cyclonic vorticity near the center, surrounded by an annulus encompassing an equal amount of anticyclonic vorticity, which consideration requires a reversed outward radial vorticity gradient. Therefore, bounded vortices must contain an outer waveguide bounded by an inner critical radius and outer turning point. 

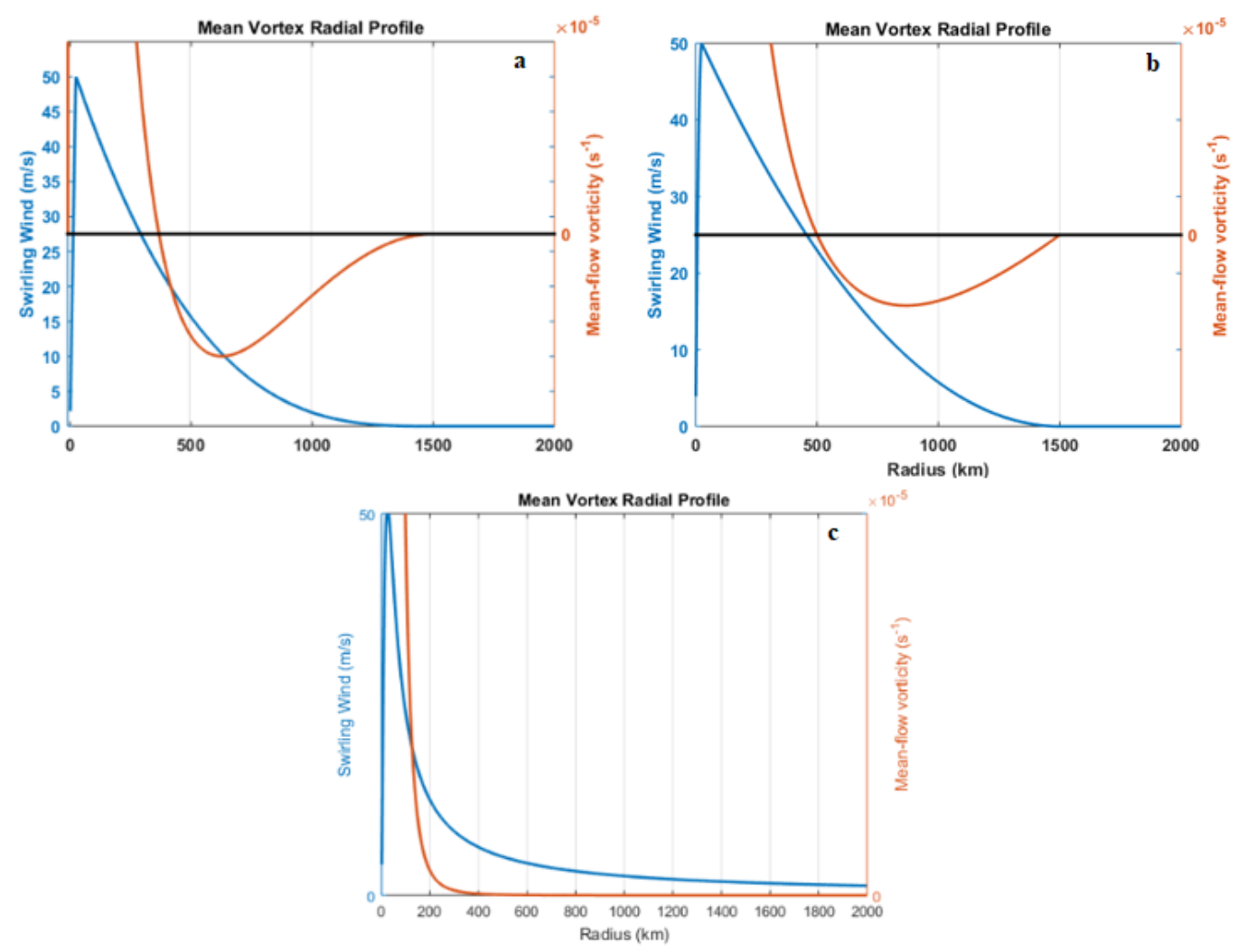

Fig 47. Radial wind and mean-flow vorticity profile for the LN3 (a), W2 (b), and unbounded (c) vortices with $50 \mathrm{~ms}^{-1}$ max winds at $25 \mathrm{~km}$.

However, bounded wind profiles raise the question: Are vorticity monopoles consistent on a closed manifold (i.e., the spherical Earth)? The answer lies in using Stokes' Theorem, which states that the circulation around an area on the surface of a manifold (any surface where Euclidean geometry applies locally) is equal to the integral of the relative vorticity over the area enclosed (Fig 48). To understand circulation on a closed manifold, consider a circular patch with radius, $R$ in solid-body rotation with angular velocity about a vertical axis, $\mathbf{\Omega}$. The circulation, $C$, is the line integral of the dot product between the wind vector, $v$ with the length differential, $d l$ around the patch's circumference (27). The integral may be rewritten with respect to azimuth angle $\lambda$, and then divided through by the area of a circle to yield the vorticity (28). 


$$
\begin{gathered}
C=\int \boldsymbol{v} \cdot d l=\int_{0}^{2 \pi}(\Omega R) R d \lambda=2 \pi \Omega R^{2} \\
\frac{C}{\pi R^{2}}=2 \Omega=\zeta
\end{gathered}
$$

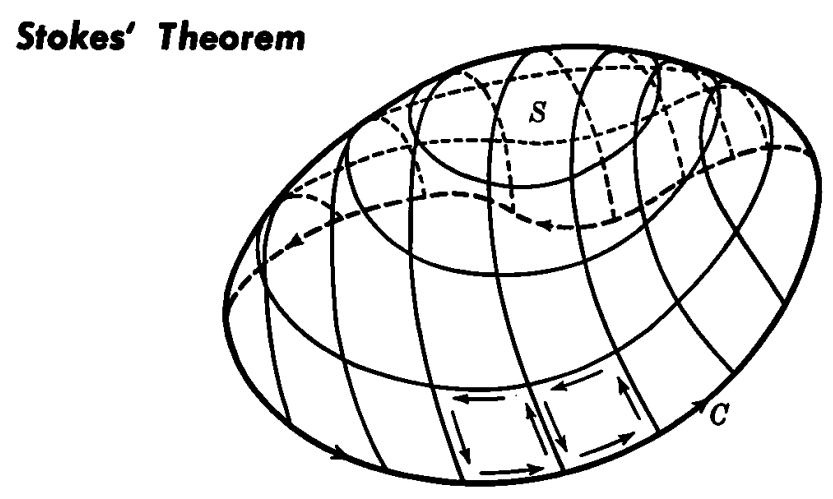

Stokes' theorem states that the surface integral of the normal component of curl $F$ over $S$ equals the line integral of the tangential component of $\mathbf{F}$ about $C$ :

$$
\iint_{S}(\operatorname{curl} \mathbf{F}) \cdot \mathbf{n} d S=\int_{C} \mathbf{F} \cdot d \mathbf{R}
$$

Fig 48. Illustration of Stokes' Theorem: Davis (1961).

To illustrate the consistency with Stokes' Theorem for bounded vortices, consider a three-dimensional atmosphere with no rigid lid. Free-slip (Fig 49) and no-slip (Fig 50) surface boundary conditions yield scenarios where vortex tubes must either terminate on the surface or reconnect. In free-slip flow, the velocity immediately above the surface is generally non-zero, but must parallel the surface. In no-slip flow, the velocity - both parallel and perpendicular to the surface - is identically zero, and vorticity is strong adjacent to the surface. The former requires vortex tubes to rise upward from the surface 
in the eyewall, spread outward somewhat below tropopause level, and then bend downward to terminate at the surface far from the vortex center. Since no-slip requires the wind at the surface to be zero, the resulting strong, near-surface shear reconnects vortex tubes in the eyewall where rising occurs. The tubes point outward and reconnect again far from the center. In either case, the vortex core where the vortex tubes rise, contains cyclonic vertical vorticity and the periphery of descent contains anticyclonic vorticity. Both boundary conditions are consistent with the properties of bounded vortices, but what about unbounded vortices? 


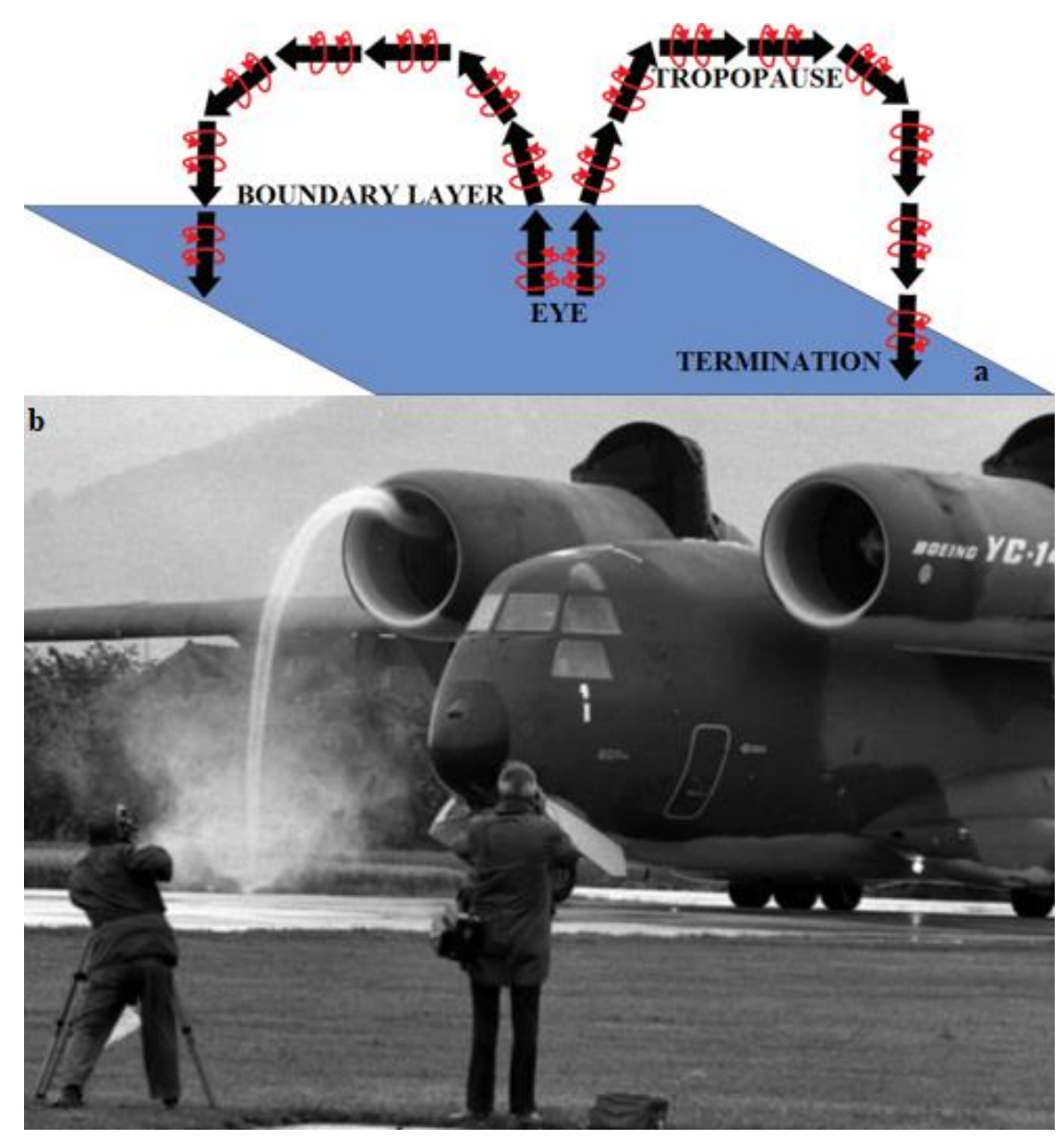

Fig 49. A free-slip boundary condition in a 3D atmosphere with no rigid lid, where vortex tubes (black arrows) rise in the vortex's eyewall to produce cyclonic vertical vorticity (red arrows) in the core, spread at tropopause level, and then subside to produce anticyclonic vorticity and terminate at the boundary-layer surface (a). An example of vortex tube surface termination (b).

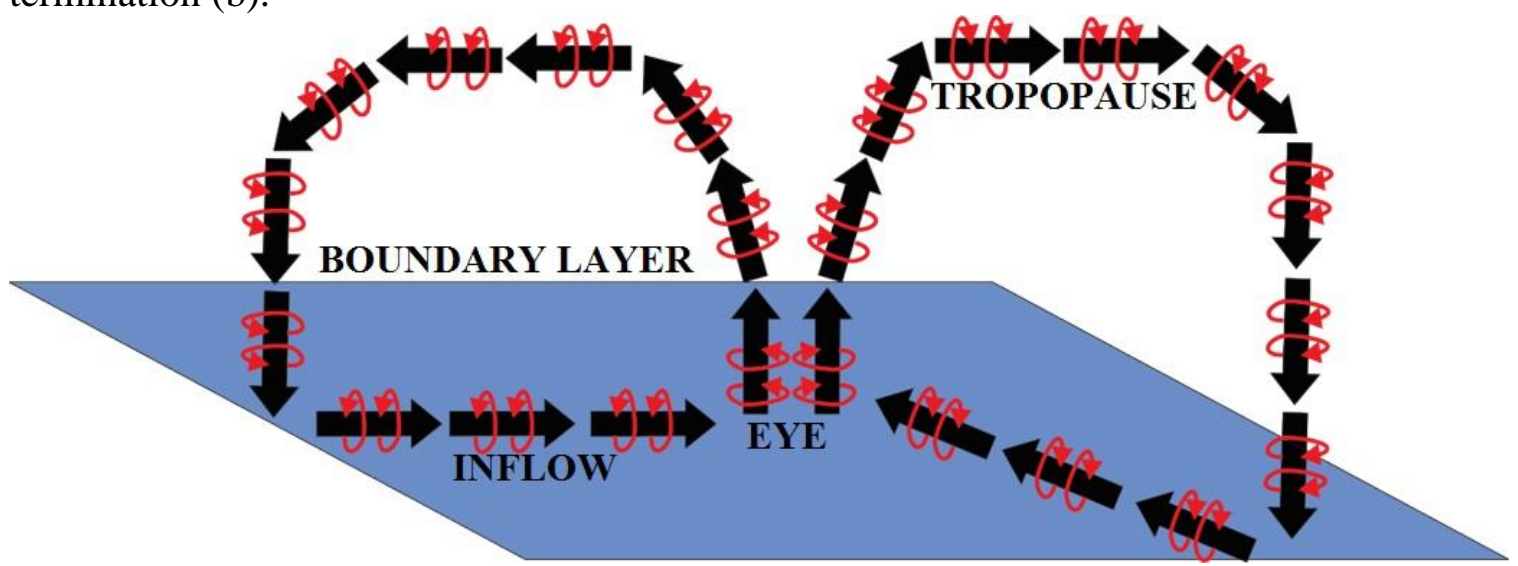

Fig 50. A no-slip boundary condition where the rising vortex tubes reconnect at the vortex periphery. 
Constraints on Parametric TC Wind Profiles

Unbounded (Fig 47c) vortices were defined by GCW as essentially smoothed Rankine vortices (e.g., Montgomery et al. 1999) with all of the vorticity concentrated in the core, a narrow "skirt" of cyclonic vorticity extending a short distance outward from the RMW, and an extensive free vortex that has constant circulation and extends to arbitrarily large radius. An unbounded vortex was used by GCW as a contrast with the asymptotically bounded, (Wood and White 2010) vortex in $\beta$-plane motion. The former is inconsistent with Stokes' Theorem on any closed manifold because unbounded wind profiles consist of solid rotation in the core surrounded by irrotational (i.e., zero vorticity) flow so that there is no outer waveguide. Moreover, unbounded vortices contain both infinite kinetic energy and infinite angular momentum. It is important to note however that the outer flow is irrotational only for a " $V / r$ profile" and that unbounded vortices may have either evenly distributed vorticity or a " $\beta$-skirt". For the purposes of the Stokes' Theorem argument, only the latter is considered.

Fundamental fluid kinematics impose physical consistency restraints on parametric wind profiles, some of which are used for idealized theoretical models or fullphysics model initialization. To further illustrate why unbounded vortices are inconsistent with Stokes' Theorem, imagine a sphere with no vorticity except for a single cyclonic patch. From the perspective of an observer at the antipode, the circulation about the antipode would appear to be anticyclonic but with no enclosed vorticity, in apparent contradiction with Stokes' Theorem (Fig 51). Keep in mind that if, viewed from the antipode, the circulation about the antipode is the mirror image of that around the center of the patch. 


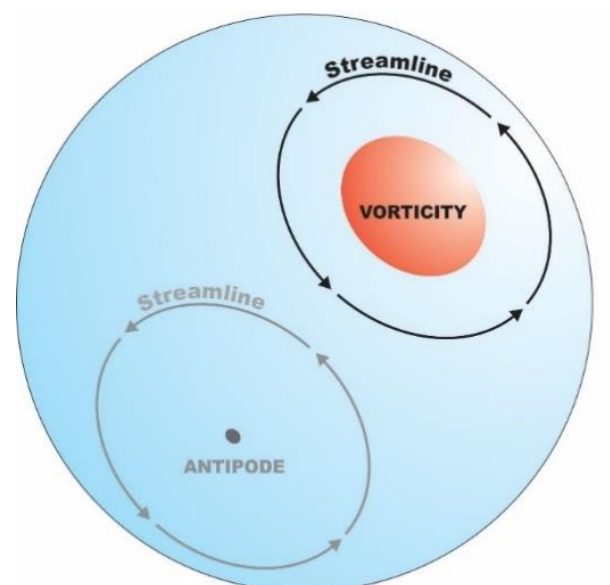

FRONT

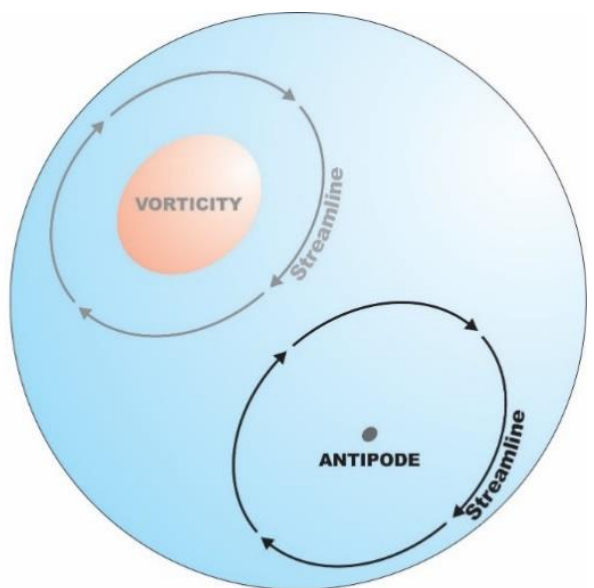

BACK

Fig 51. Illustration of a cyclonic vorticity patch on a sphere with no vorticity anywhere else and the apparent anticyclonic circulation at the patch's antipode, but with no enclosed vorticity.

The contradiction may be avoided by the presence of diffuse anticyclonic vorticity everywhere outside the patch or a relatively narrow annulus of anticyclonic vorticity around the patch. In either case the component of the curl normal to the surface of any closed manifold must integrate to zero. The enclosed vorticity increases to a maximum at the boundary of the vortex core as the integral (29) starting from the patch's center, expands, where $d A$ and $d \theta^{\prime}$ are the area and angle differentials, respectively. Then from the boundary to the antipode, the path length $(R \sin \theta)$ initially increases until the angle reckoned from the patch center is $\theta=\pi / 2$. The sphere's geometry shortens the path afterward. Therefore, to keep the circulation constant, the wind, $V(\theta)$, must increase even though no more vorticity is enclosed. At the antipode, $R \sin \theta=0$, causing a singularity in which $V(\theta)$ becomes infinite (Fig 52):

$$
\iint \zeta d A=\int_{0}^{\theta} \zeta\left(2 \pi R \sin \theta^{\prime}\right) R d \theta^{\prime}
$$




$$
\begin{gathered}
V(\theta)(2 \pi R \sin \theta)=2 \pi R^{2} \zeta(1-\cos \theta) \\
V(\theta)=\frac{R \zeta(1-\cos \theta)}{\sin \theta} \\
V(\theta)=\frac{R \zeta\left(1-\cos \pi_{0}\right)}{\sin \pi}
\end{gathered}
$$

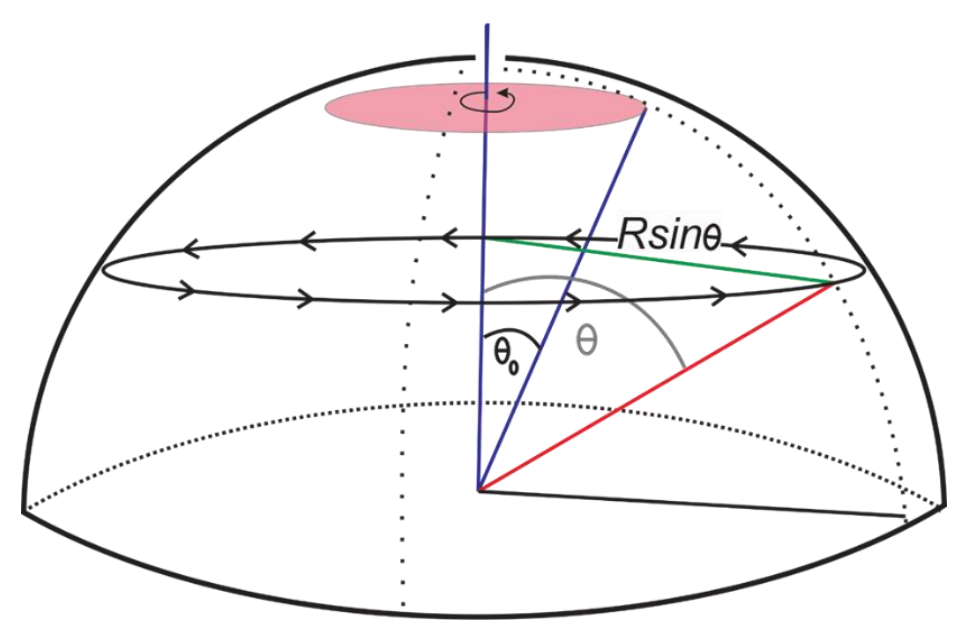

Fig 52. Integrating from the center of the cyclonic vorticity patch outward to the boundary and cross-equatorially toward the antipode which results in a singularity where the wind must approach infinity to keep the circulation constant.

Bounded and Unbounded Vortex Modeling Applications

\section{Recent Work}

Analysis of bounded and unbounded vortices has implications for idealized TC motion modeling. For example, GCW showed that in a linear, wavenumber-1 BND model on a $\beta$-plane, either an asymptotically bounded or unbounded vortex yielded northwestward motion two to three times faster than the observed, $1-2 \mathrm{~ms}^{-1} \beta$-drift. The fast motion and large asymmetry amplitude of the unbounded vortex were attributed to 
too-strong forcing from advection by the outer irrotational portion of the broad circulation (Fig 53). Finitely bounded vortices exhibited somewhat slower northwestward motion because of the narrower outer waveguides from a more compact circulation. Beyond the $1500-\mathrm{km}$ "bounding radius", the area-integrated symmetric vorticity was exactly zero. The translation speed varied as a function of the shape of the positive radial vorticity gradient profile at the vortex periphery (Fig 53c,d).

Diffusion and especially nonlinearity were actually the dominant factors in controlling translation speed. Fourth-order diffusion applied to velocity (e.g., Montgomery et al. 1999) resulted in the filamented flow near the critical radius limiting the vortex translation speed. Second-order diffusion applied to vorticity had a similar effect. Wave-wave interaction however, was the primary mechanism in nonlinear semispectral models where the wavenumber-1 anti- $\beta$-gyre solution (refer to Fig 39) produced a southeastward counterflow that opposed the linear beta gyres' northwestward flow across the vortex center to limit the overall storm motion. 

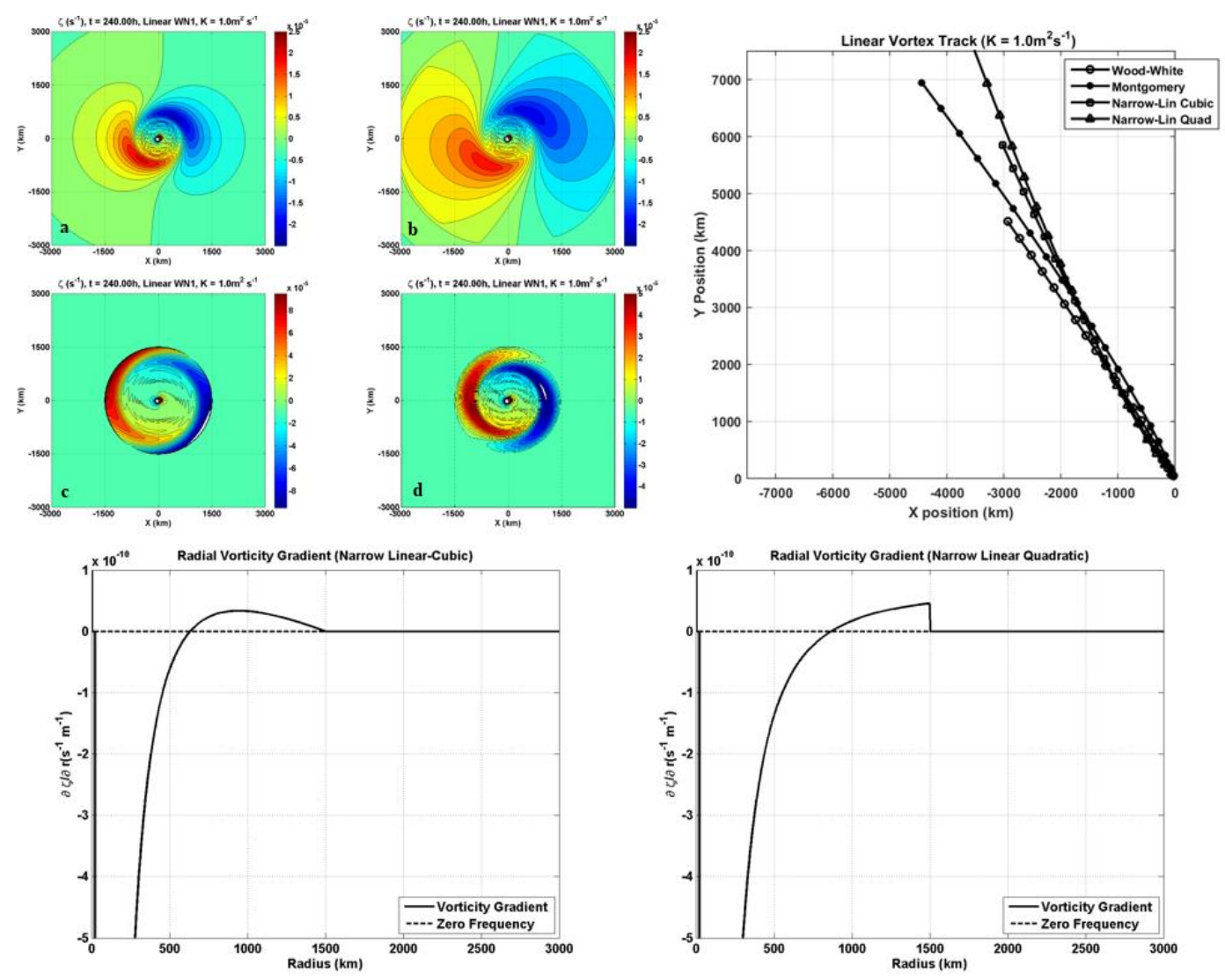

Fig 53. Beta-gyre vorticity asymmetries (upper left) for an asymptotically bounded and unbounded vortex $(\mathrm{a}, \mathrm{b})$ and two finitely bounded vortices $(\mathrm{c}, \mathrm{d})$; northwestward beta-drift for each vortex (upper right); radial vorticity gradient for the finitely bounded vortices (bottom row): Gonzalez et al. 2015.

The remainder of Chapter 5 builds upon GCW by introducing two finitely bounded vortices and one unbounded vortex to the present model to evaluate how the inner and outer waveguides affect vorticity, streamfunction, and vortex motion. The finitely bounded wind profiles used are defined by the radial profile width, the power-law used to set the outer-vortex shape, and the $250-\mathrm{km}$ bounding radius. The justification for the small bounding radius stems from NM99 who noted the difficulty in seeing how the inner-core dynamics should be changed by the structure of the mean azimuthal velocity in the far field. Although it was shown that unbounded vortices are inconsistent with 
Stokes' Theorem, an unbounded profile is used nonetheless to provide further comparisons among different wind profiles. To yield fair comparisons, the other vortices will also have a $50 \mathrm{~ms}^{-1}$ maximum wind, with the forcing imposed at the $25-\mathrm{km}$ RMW, and rotating with orbital period, $T=3.5 \mathrm{hrs}$.

\section{Narrow-Linear-Cubic Profile}

Bounded vortices have piecewise-continuous structures and solid rotation near the center as defined by GCW,

$$
V(R)=V_{2} \frac{R}{R_{2}}, 0 \leq R \leq R_{2}
$$

where $V(R)$ is the wind at a given radius, and $V_{2}$ and $R_{2}$ are the wind and radius at the inner transition, respectively. Next, $V$ is computed in the transition across the RMW $\left(R_{o}\right)$ with a cubic interpolating polynomial:

$$
V(R)=\frac{V_{x}}{R_{o}^{2}}\left(-4 R^{2}+8 R_{o} R-3 R_{o}^{2}\right), R_{2} \leq R \leq R_{1}
$$

Here, $V_{x}$ is the $50 \mathrm{~ms}^{-1}$ maximum wind, and $R_{1}$ is the radius at the outer transition. Then, the cubic power-law outer wind profile is: 


$$
V(R)=V_{1}\left(\frac{R_{9}-R_{0}}{R_{9}-R_{1}}\right)^{3}, R_{1} \leq R \leq R_{9}
$$

Here, $V_{1}$ is the wind at the outer transition and $R_{9}$ is the $250-\mathrm{km}$ bounding radius.

Beyond, $R_{9}$, the wind is zero. From the eye outward to the RMW, $V$ increases sharply, followed by an abrupt transition across the RMW, and a slow decay with radius (Fig 54a). By contrast, the asymptotically bounded wind profile (hereafter, WW) used for the default mean vortex has a smoother RMW transition between the eye and a more rapid decay with radius. Nevertheless, the LN3-vortex has a very narrow inner waveguide. Between 20 and $30 \mathrm{~km}$, there is a sharp cutoff-frequency gradient, followed by an asymptotic decrease to zero toward $100 \mathrm{~km}$. The LN3 waveguide supports a range of orbital periods where VRW propagation is between an essentially fixed 20-km turning point and critical radius as great as $\sim 60 \mathrm{~km}$ (Fig 54b).

Forced $\zeta$ asymmetries are very narrow and elliptical, surrounded by a large symmetric ring of highly filamented, interlocked vorticity at the $\sim 60-\mathrm{km}$ critical radius. Additional filaments appear to pool in the eye to form a cross-like pattern (Fig 54c). Streamfunction shows the expected pattern of small, inner convectively forced gyres surrounded by large, outer gyres forced by the orbital motion of the vortex. However the vortex's small circulation gives the outer gyres a compressed appearance (Fig 54d). Although the general $\zeta$ and $\psi$ patterns are not much different than for the WW-vortex, vortex motion is much different. The vortex center interestingly performs an east-of-duenorth drift with superimposed trochoidal wobbles ( $\sim 7$ orbits), at a $\sim 2 \mathrm{~ms}^{-1}$ mean speed (Fig 54e,f). The drift is in the same direction as the initial motion after start-up transient 
growth. A possible explanation for the "odd" motion is that the orientation of the dipole from the point where the dipole stabilizes shortly after start-up transient growth, advects axially symmetric vorticity to the northeast that causes the vortex center to drift in the same direction. However, the finitely bounded vortex has a local radial vorticity gradient sign reversal near $100 \mathrm{~km}$, suggesting that there is a weak barotropic instability that may sustain the start-up transient drift for the remainder of the simulation. The next finitely bounded vortex exhibits similar behavior. 

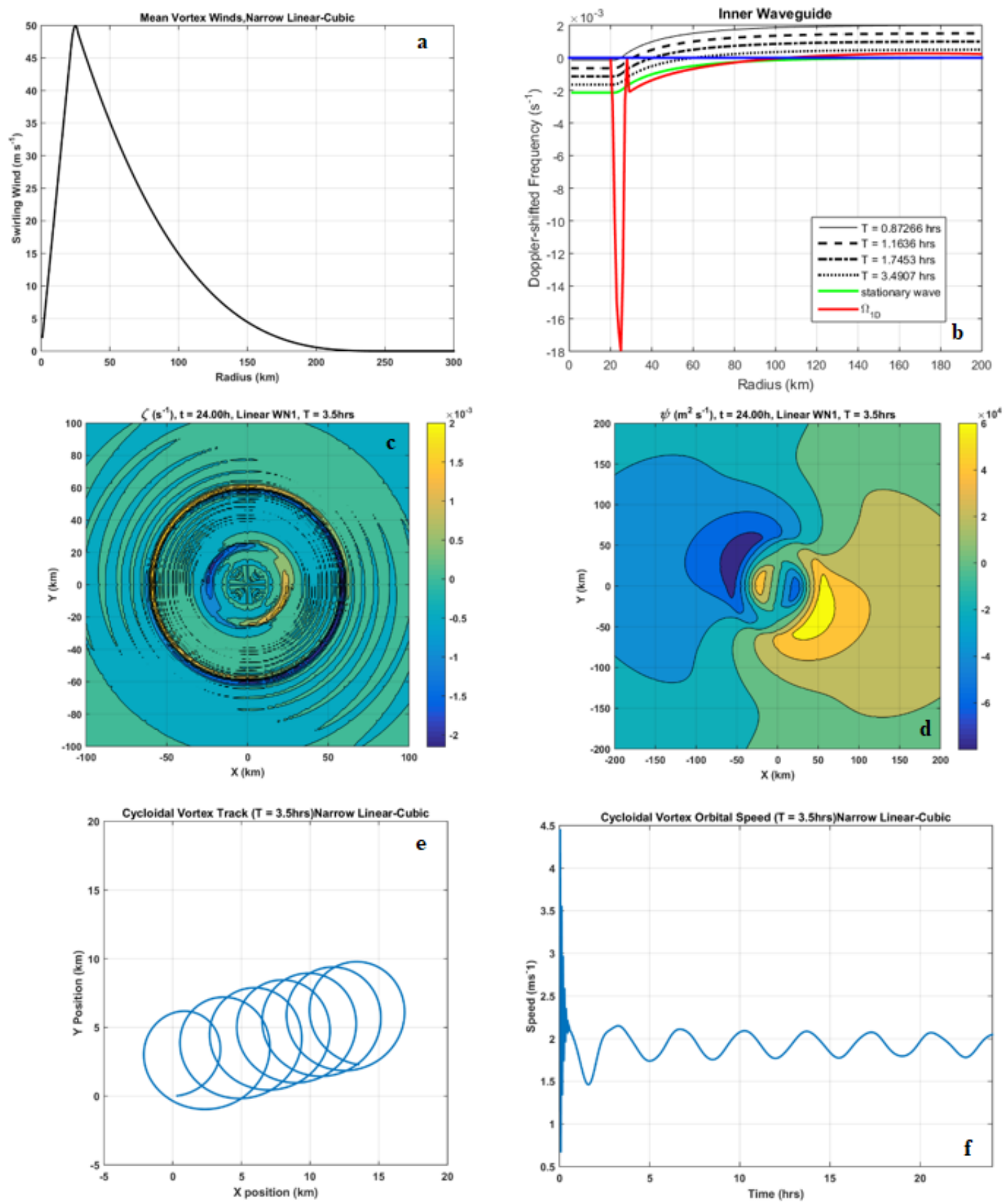

Fig 54. LN3 vortex radial profiles (top row), forced wavenumber-1 dipole (mid row), and vortex motion (bottom row). 
Wide-Quadratic Profile

The wide-quadratic (hereafter W2) profile is obtained in much the same way as LN3, but with different RMW transition, and the outer power-law exponent (37), is two instead of three:

$$
\begin{aligned}
V(R)= & \frac{V_{x}}{R_{o}^{2}}\left(2 R_{o} R-R^{2}\right), R_{2} \leq R \leq R_{1} \\
& V(R)=V_{1}\left(\frac{R_{9}-R_{0}}{R_{9}-R_{1}}\right)^{2}, R_{1} \leq R \leq R_{9}
\end{aligned}
$$

The gradient wind increases linearly from the center, outward to the RMW, followed by a sharp RMW transition and slow decay beyond. The transition appears as a blend between the convex curve of the WW-profile and the sharp angular curve of the LN3-profile (Fig 55a). As the name suggests, the W2-vortex has a wide waveguide where the geometry of the $\Omega_{1 \mathrm{D}}$ curve becomes sharply negative from the center, outward to $R_{Q}$, resulting in more flexible turning points. Similar to LN3, the W2 waveguide enables VRW propagation outward to a large critical radius. For $T=3.5 \mathrm{hrs}$, the W2vortex critical radius is $\sim 65 \mathrm{~km}$ (Fig 55b). Vorticity, streamfunction, and motion (Fig55c-f) are similar to the LN3 vortex. The latter reinforces that the eastward oscillating drift is an artifact of the bounded vortices' local vorticity gradient reversal that results in a weak barotropic instability that may sustain the translation after start-up transient growth of the dipole. 

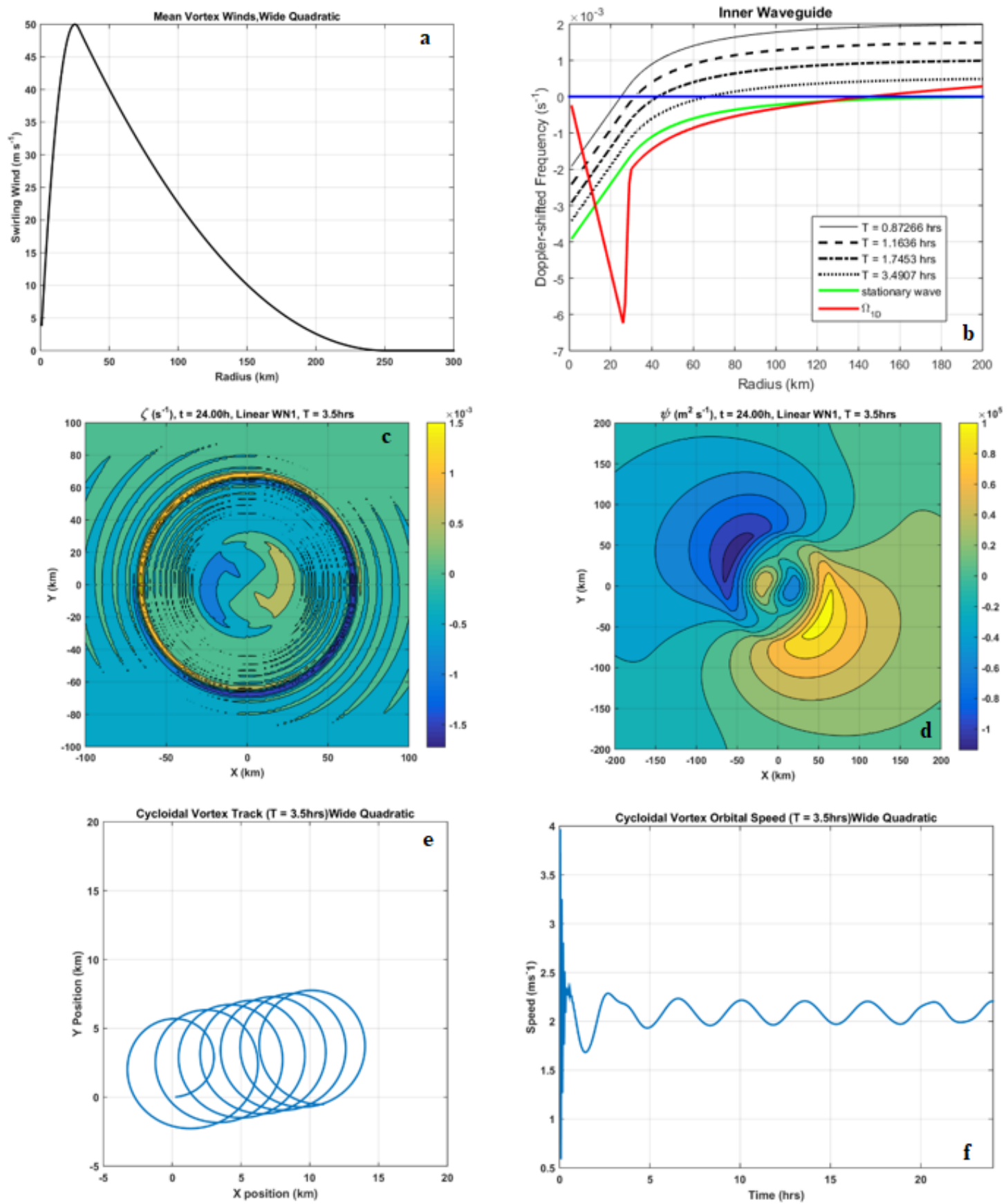

Fig 55. W2 vortex radial profiles (top row), forced wavenumber-1 dipole (mid row), and vortex motion (bottom row). 


\section{Unbounded Profile}

The formulation for the unbounded wind profile, is nearly the same as the WWvortex (Chapter 2, equation 1), but differs in the outer power-law exponent $\left(N_{\text {out }}=-1\right)$ and transition width parameter $(L=1)$. Additionally, the calculated cyclostrophic wind, $V_{c}$ is set to be the same as the gradient wind, $V$, just as in GCW:

$$
V_{c}(r)=V_{c x}\left\{\frac{\left(\frac{r}{R_{x}}\right)^{\frac{N_{\text {in }}}{L}}}{1+\frac{N_{\text {in }}}{N_{\text {in }}-N_{\text {out }}}\left[\left(\frac{r}{R_{x}}\right)^{\frac{N_{\text {in }}-N_{\text {out }}}{L}}-1\right]}\right\}^{L}=V_{c x}\left\{\frac{\frac{r}{R_{x}}}{1+\frac{1}{2}\left[\left(\frac{r}{R_{x}}\right)^{2}-1\right]}\right\}=V
$$

The gradient wind experiences a smooth transition across the RMW, followed by an inverse $r$ decay with radius that does not go to zero at any finite radius (Fig 56a), nor is there any vorticity sign reversal. The range of passbands is comparable with the WWvortex showing a critical radius $\sim 65 \mathrm{~km}$ for the shortest selected $T$ within the passband range. Turning point radii are $\leq 10 \mathrm{~km}$ however, yielding a waveguide $45 \mathrm{~km}$ wide (Fig 56b). Vorticity asymmetries have a horseshoe-like shape with highly filamented $\zeta$ between the forced dipole and the outer symmetric vorticity ring (Fig 56c). Unlike the

finitely bounded vortices, the unbounded vortex center's trochoidal motion does not drift at all and the mean orbital speed is slightly slower, at $\sim 1.7 \mathrm{~ms}^{-1}$ (Fig 56e,f). In fact, the unbounded vortex motion similar to the WW-vortex. Table 7 summarizes the main results for all three vortices discussed. 

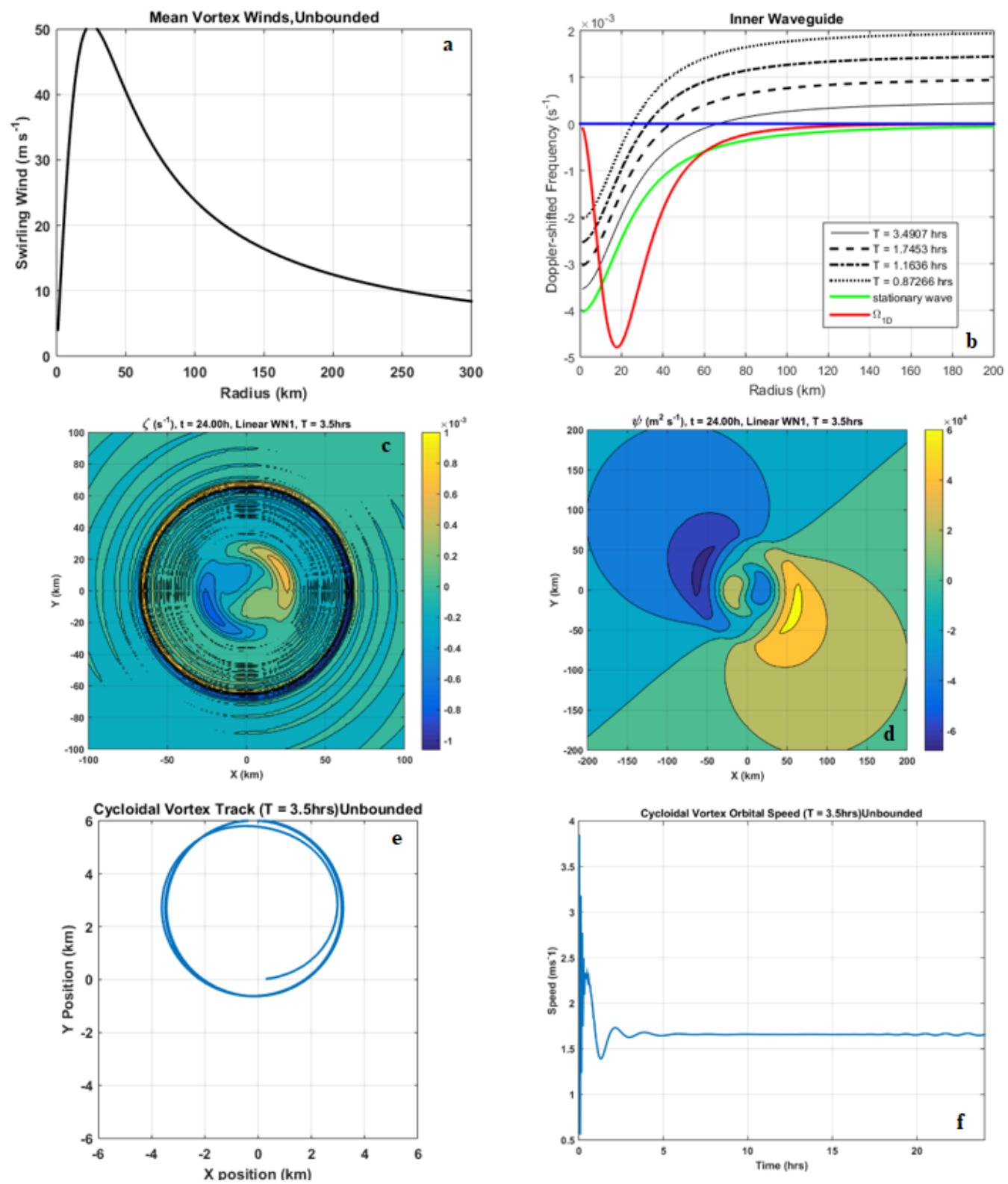

Fig 56. Unbounded vortex radial profiles (top row), forced wavenumber-1 dipole (mid row), and vortex motion (bottom row). 
Table 7. Waveguide width and vortex-motion results for finitely bounded vortices and an unbounded vortex (3.5-hr orbital period).

\begin{tabular}{|c|c|c|c|}
\hline $\begin{array}{c}\text { Waveguide width } \\
(\mathbf{k m})\end{array}$ & LN3 vortex & W2 vortex & Unbounded vortex \\
\hline $\begin{array}{c}\text { Mean orbital } \\
\text { speed (ms }\end{array}$ & 2 & 65 & 57 \\
\hline Orbital behavior & $\begin{array}{c}\text { Oscillating east- } \\
\text { northeast drift }\end{array}$ & $\begin{array}{c}\text { Oscillating east- } \\
\text { northeast drift }\end{array}$ & $\begin{array}{c}\text { Non-drifting } \\
\text { trochoidal motion }\end{array}$ \\
\hline
\end{tabular}

Summary

Sensitivity studies of vortex intensity and wind profiles yield insight into TC motion, inner waveguide dynamics, and wave propagation. Initial experiments analyze mean vortices of tropical depression, tropical storm, and category-5 intensity. Consistent with observed climatology, the maximum wind, radius of maximum wind, and outer power-law exponent are adjusted simultaneously. Weak vortices are initialized with large eyes, flat wind profiles, and reduced forcing amplitudes. Although the inner waveguide is wider, the weaker vortices exhibit minimal vortex Rossby wave propagation because of the smaller forcing amplitude and weak radial vorticity gradient. Despite limited wave activity, weak vortices demonstrate fast motion, consisting of unrealistically broad trochoidal tracks, caused by the large radius of maximum winds, which creates weaker vorticity between the eye and eyewall.

The category-5 vortex has a smaller eye, sharper wind profile, and stronger forcing. Forced waves propagate upon a stronger vorticity gradient within a narrower waveguide. A small ring of filamented vorticity consequently forms in the neighborhood 
of the critical radius, with a pronounced wake that aligns with the vortex's motion, outward from the ring. The rapidly rotating, mass source-sink pair is close to the vortex center, which causes a tight trochoidal track. Despite the differences in results from all 3 vortex intensities, the most significant findings are that the inner waveguide width is affected by changes to RMW and the outer power-law exponent, and weak vortices exhibit fast motion because the RMW is large. However, the vorticity gradient and forcing amplitude must be strong to induce significant VRW propagation. Therefore, mature-to-strong vortices consistently exhibit trailing spirals at the critical radius from outward-propagating waves that appear as tightly wound vorticity filaments.

Waveguide geometry further motivates examination of vortices that have different wind profiles. Finitely bounded vortices' circulation becomes identically zero at a large, but finite radius. In a 3-dimensional vortex, under a no-slip boundary condition, horizontal vortex tubes would rise in the eye and eyewall, spread outward in the vertical shear just below the tropopause, descend and reconnect to produce anticyclonic vertical vorticity at the bounded vortex's periphery. Thus, finitely bounded vortices are consistent with Stokes' Theorem and therefore a valid choice to simulate TC dynamics accurately with semi-spectral models. In both finitely bounded vortices, the waveguide becomes narrower in a more compact circulation, but exhibits very similar vorticity and streamfunction fields. The finitely bounded vortices exhibited trochoidal motion on an $f$ plane but with an east-northeastward drift that may be attributed instability caused by a local vorticity gradient sign reversal well inward from the bounding radius, such that the drift is sustained after start-up transient growth of the dipole. 
Chapter 5 also highlights the limitations of unbounded vortices. If one considers a single, cyclonic vorticity patch on a closed, spherical Earth, surrounded by zero vorticity everywhere else, then the patch's antipode has an anticyclonic circulation around a contour that encloses zero vorticity, which contradicts Stokes' Theorem. More generally, the component of the curl normal to the surface of any closed manifold must integrate to zero over the whole surface. A way around the contradiction is to have diffuse anticyclonic relative vorticity outside the patch. If there is net vorticity, a singularity arises around the antipode where the wind approaches infinity as the path length approaches zero, keeping the circulation constant. In addition to the circulation inconsistency, unbounded vortices have infinite angular momentum and infinite kinetic energy on a Cartesian plane. Despite the arguments against unbounded profiles, experiments with unbounded vortices still advance understanding of small-scale TC motion induced by VRWs in the inner waveguide. The unbounded waveguide structure and critical radius for a given orbital period are more-or-less comparable with an asymptotically bounded vortex. The next chapter applies "waveguide thinking" to synoptic-scale Rossby waves in a horizontally sheared zonal flow in which the versatility of a barotropic, nondivergent framework is showcased by simulating frontal cyclones that characterize mid-latitude weather. 


\section{CHAPTER VI. SYNOPTIC-SCALE ROSSBY WAVES IN A MERIDIONALLY SHEARED, ZONAL FLOW}

\section{General Overview}

The previous chapters synthesized wavenumber-1 VRW dynamics in terms of TC-like vortex motion, waveguides, and eddy fluxes using a BND model. Chapter 6 demonstrates the model's versatility by extending the framework to a mid-latitude beta plane and exploring analogous, synoptic-scale Rossby waves. As stated previously, VRWs were first suggested by MacDonald (1968) when he noticed that TC spiral bands' orientation with respect to the storm's axis of rotation was similar to that of synopticscale troughs with respect to the Earth's axis of rotation (refer to Fig 10). The troughs are classic Rossby waves - planetary-scale perturbations that propagate upon the Earth's meridional planetary vorticity gradient. Rossby waves play a key role in jet stream dynamics, frontal cyclone development, and large-scale momentum transports. Here, the approach used to understand VRWs is repurposed, to the synoptic-scale, mid-latitude atmosphere to yield a conceptually simple understanding of classical Rossby waves.

\section{Background}

\section{Early History}

Rossby waves are named after the pioneering Swedish meteorologist, Carl-Gustaf Arvid Rossby (Fig 57a), who described large-scale atmospheric motions and derived 
linearized equations that govern waves in the mid-latitude westerlies (e.g., Rossby et al. 1939 and Rossby 1940). Rossby presented maps of westerly circumpolar winds in the Northern Hemisphere whose streamlines consisted of southward "bulging lobes" (Fig 57b). He argued that the lobes were waves that shifted positions at speeds that depended on the circumpolar wind, the length of the waves, and the beta effect. The latter influences the spin of air masses moving southward from higher latitudes. Overall, Rossby's descriptions were a scientific breakthrough in meteorological thought because mid-latitude weather follows the waves' ever-shifting positions around the Earth. Subsequent studies followed and further advanced understanding of Rossby waves' propagation, momentum transport, and interaction with the large-scale, synoptic flow.

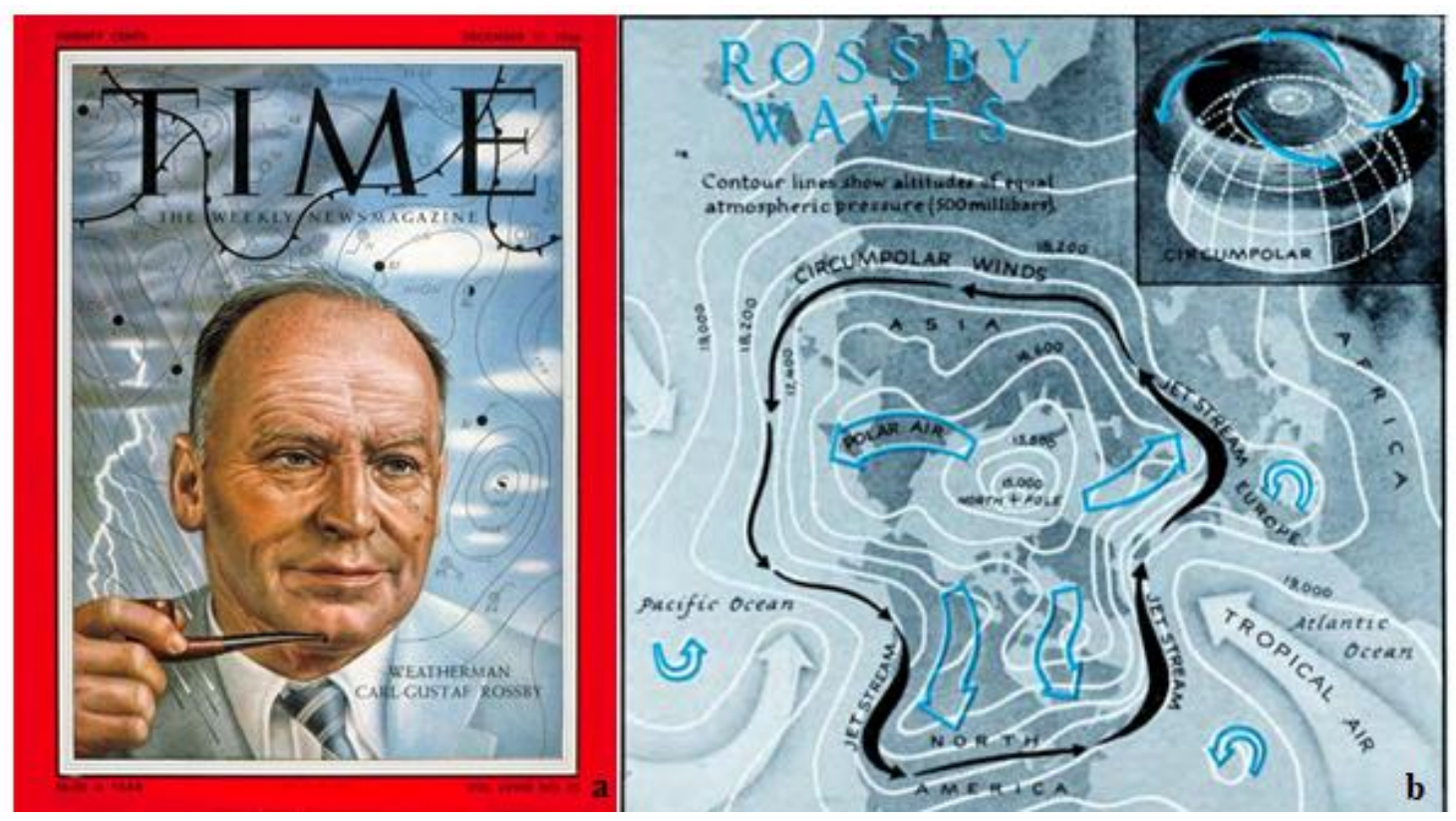

Fig 57. Carl-Gustaf Rossby and an interpretation of his waves with respect to the Earth's circumpolar winds and the jet stream: Segund (1956). 
Propagation

Barotropic Rossby waves propagate horizontally with zonal and meridional phase and group velocities that depend only on $\beta$ and wavenumbers. Randal and Held (1991) deduced that meridional propagation is primarily toward lower latitudes and results in barotropic decay of waves. Chapter 6 aims to capture Rossby-wave propagation within a meridional waveguide to understand what occurs at different latitudes. Held and Phillips (1995) found in a barotropic model that the divergence of eddy momentum flux from low latitudes is a direct consequence of potential vorticity mixing by Rossby waves propagating into the Tropics from mid-latitudes, analogous to the angular momentum flux divergence at the critical radius of the VRW inner waveguide. The Rossby-wave momentum flux budget is also clarified here. More recently, Lee et al. (2007) noted that the initiation of the poleward propagation is marked by the formation of negative zonal wind (i.e., easterly) anomalies in the Tropics. In addition, anomalies arise from meridional overturning or breaking of waves that originate in lower latitudes. Lastly, equatorward radiation of mid-latitude waves is halted (i.e., absorbed), which results in wave breaking at the poleward end of the homogenized potential vorticity region. In summary, Rossby waves propagate both poleward and equatorward, which have implications for momentum transport.

\section{Momentum Transport}

Rossby waves are eddies that transport energy and momentum. A model for transient eddy momentum fluxes in the upper troposphere was developed by DeSole (2001) who concluded the following: 
- Dominant mid-latitude eddies grow primarily by extracting available potential energy from the mean flow.

- Observed eddies often transport angular momentum toward latitudes of stronger angular velocity. The same is true for VRWs that converge angular momentum inward toward the high orbital velocity of the vortex core.

- Quasigeostrophic (i.e., including advection of planetary vorticity but with fixed $f_{\mathrm{o}}$ at $45^{0}$ latitude for commuting balanced flow) waves transport angular momentum in the direction opposite to the meridional group velocity. Thus the flux of westerly momentum is opposite to wave energy propagation in Northern Hemisphere winter. (Lorenz and Hartmann 2003). By analogy, VRWs propagate radially outward with a positive group velocity from the vortex core, but transport angular momentum inward.

- If Rossby-wave-activity generation is localized at a given latitude, the eddy momentum flux will converge at the source.

- Momentum fluxes are more strongly controlled by the radiation of Rossby waves from low levels than by background flow.

To summarize, synoptic-scale momentum fluxes are controlled by multiple factors that include latitude of generation, wave propagation direction, and latitude of strongest zonal wind, most of which can be represented in a barotropic, nondivergent context. Therefore large-scale, environmental flow plays a crucial role in understanding Rossby-wave transport dynamics. 


\section{Mean-Flow Interactions}

Modeling Rossby waves in meridionally varying zonal flows yields important insight into the ways that forced eddies interact with horizontal shears or jets. Like VRWs, Rossby waves propagate within waveguides that are bounded by "critical latitudes/levels" (analogous to the critical radius) and turning points. James (1987) connected the expression of the structural alteration of the normal modes to the morphology of the "steering" or critical level (Geisler and Dickinson 1974), where the basic zonal flow speed is equal to the phase speed of the unstable wave. Recall that VRWs absorbed at the critical radius are Doppler-shifted to zero frequency so that the specified rotation frequency equals the TC's mean-flow angular velocity $(\omega=V / r)$. Eddies with slower zonal phase speeds tend to propagate further into regions of weaker mean flow. Chapter 3 demonstrated that free-propagating VRWs with a low specified frequency (slow phase speed) were absorbed at large critical radii where the vortex mean flow is much weaker than in the source region. The waves also mix potential vorticity and decelerate the mean flow at about $10-20^{\circ}$ from the critical latitude (Randal and Held 1991). If eddies are forced by heat sources in the Tropics, then changes in mean zonal wind in the Tropics lead to a latitudinal shift in the divergence of the angular momentum pattern (Chang 1998). For jet flows, a westerly, mid-latitude jet is a waveguide for external Rossby waves that tend to propagate into and remove momentum from the jet. Thus, the jet attracts wave activity (Lorenz and Hartmann 2003).

Barotropic, nondivergent Rossby waves in a linear, horizontally sheared flow are the focus of Chapter 6. Here, the analogy with VRWs is explored while also demonstrating the BND framework's versatility. The waves' behavior is analyzed, the 
dynamical properties of the Rossby waveguide are considered, eddy flux budgets are examined to understand large-scale momentum and energy transport, and a few sensitivity studies are conducted to determine what parameters most influence the critical latitude. To achieve the desired objectives, the VRW model from previous chapters is repurposed on a mid-latitude $\beta$-plane, and is described in greater detail in the next section.

Formulation

\section{Model Overview}

The analysis of synoptic-scale Rossby waves is similar to that for VRWs, but in a Cartesian coordinate system. The model, written in MATLAB, reads the shear-flow profile and wave forcing parameters from a setup file, and solves for the streamfunction $\psi$ in a frequency-wavenumber domain to realize the structure in $x-y$ space. The moving wavetrain is then analyzed to produce eddy momentum and geopotential fluxes. The mid-latitude wind profile is represented in a 4000-km meridional domain with a horizontally sheared, zonal flow, $U(y)=S\left(y-y_{0}\right)$, where $S$ is the shear, $y$ is the meridional distance, and $y_{0}(500 \mathrm{~km})$ is the meridional coordinate of the transition from easterly to westerly mean flow. The mostly mid-latitude domain extends from $20^{\circ} \mathrm{N}$ to $60^{\circ} \mathrm{N}$. The mean flow is linearly sheared, consisting of weak easterly winds south of $25^{\circ} \mathrm{N}$, and increasingly strong westerly winds that peak at $9 \mathrm{~ms}^{-1}$ at $60^{0} \mathrm{~N}$ (Fig 58a). To excite the waves, a 50-km wide sinusoidal vorticity forcing with a polynomial Bell function meridional structure is imposed near the center of the domain (Fig 59). 


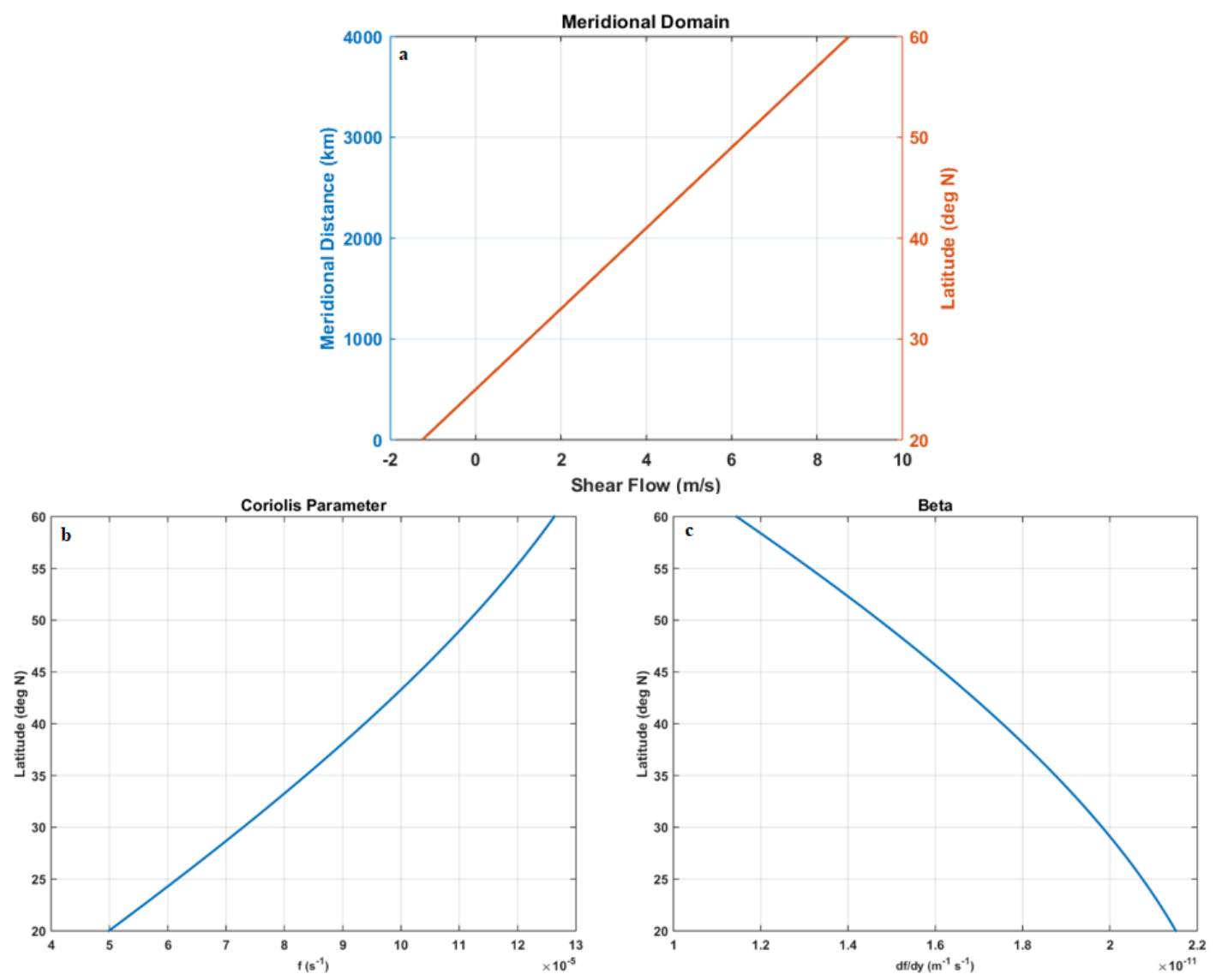

Fig 58. Meridional profiles for mean flow (a), planetary vorticity (b), and beta (c).
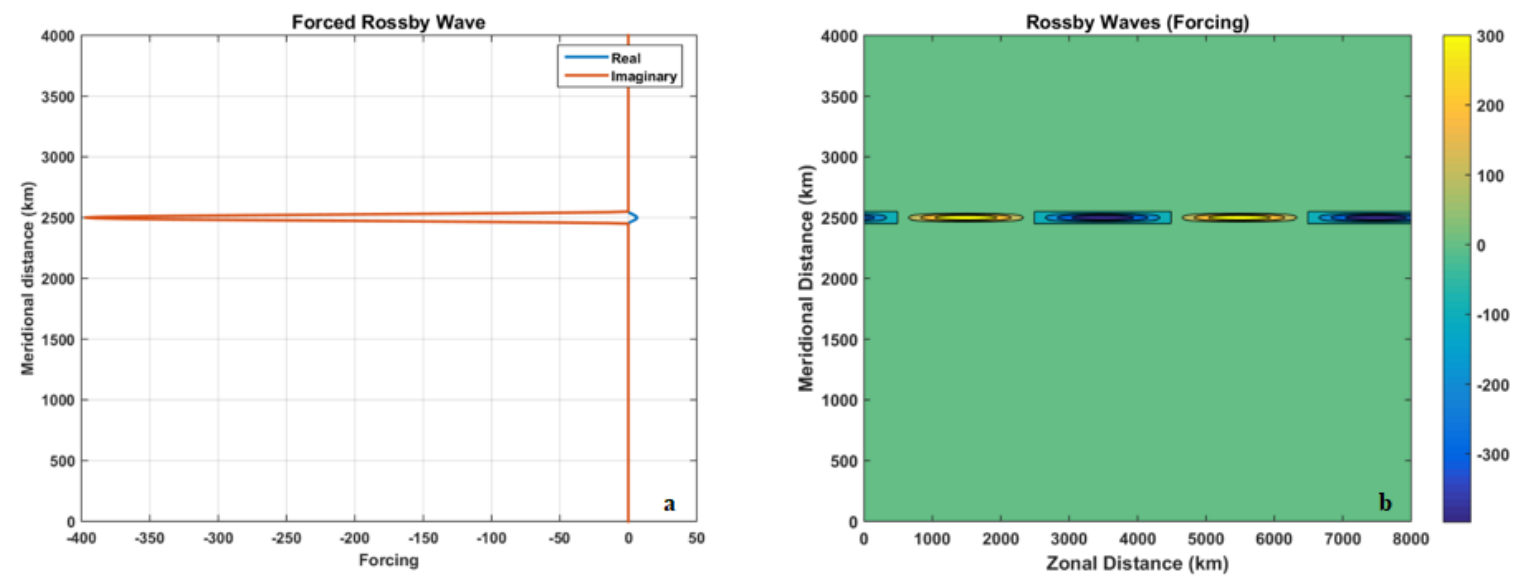

Fig 59. Imposed forcing's meridional structure (a) and contour plot (b) near the middle of the domain. 
Vorticity Equation

As in Chapter 2, vorticity $(\zeta=\partial v / \partial x-\partial u / \partial y)$ is the essential variable. Solution of the vorticity equation is the key to obtaining streamfunction $\psi$, to model Rossby-wave structure. The derivation begins with the zonal and meridional momentum equations in an imposed mean shear.

$$
\begin{gathered}
\left(\frac{\partial}{\partial t}+U \frac{\partial}{\partial x}\right) u+(S-f) v=-\frac{\partial \phi}{\partial x}+F_{u} \\
\left(\frac{\partial}{\partial t}+U \frac{\partial}{\partial x}\right) v+f u=-\frac{\partial \phi}{\partial y}+F_{v}
\end{gathered}
$$

The first terms in parenthesis are linearized Lagrangian derivatives, $S=\partial U / \partial y=$ $2.5 \times 10^{-3} \mathrm{~s}^{-1}$ is the meridional shear, $f v$ and $f u$ represent the Coriolis force. On the righthand side are the geopotential gradients and momentum forcing. The latter are derived from a vector forcing potential, $A$ such that, $F_{u}=-\partial A / \partial y$ and $F_{v}=\partial A / \partial x$. Taking $-\partial / \partial y$ of (39) and $\partial / \partial x$ of (40) followed by subtraction and recognizing that $\beta$ varies only with $y$, eliminates the geopotential $\phi$ to yield the nondivergent, vorticity equation, where $Q$ is the imposed forcing, equal to the Laplacian of $A$ :

$$
\left(\frac{\partial}{\partial t}+U \frac{\partial}{\partial x}\right)\left(\frac{\partial v}{\partial x}-\frac{\partial u}{\partial y}\right)+\beta v=\frac{\partial^{2} A}{\partial x^{2}}+\frac{\partial^{2} A}{\partial y^{2}} \equiv Q
$$




\section{Streamfunction and Geopotential Solutions}

The nondivergent flow is represented in terms of $\psi$, such that the horizontal velocity components are $u=-\partial \psi / \partial y$ and $v=\partial \psi / \partial x$ :

$$
\left(\frac{\partial}{\partial t}+U \frac{\partial}{\partial x}\right)\left(\frac{\partial^{2} \psi}{\partial x^{2}}+\frac{\partial^{2} \psi}{\partial y^{2}}\right)+\beta \frac{\partial \psi}{\partial x}=0
$$

The $2^{\text {nd }}$ factor in (42) is the Laplacian of $\psi$. Assuming frequency/wavenumber-domain solutions for $\psi(x, y, t)$ yields, $\operatorname{Re}\left\{\Psi(y) e^{[i(\omega t-k x)]}\right\}$. Here, $\omega$ is the specified propagation frequency, $k$ is the fixed zonal wavenumber, and $\Psi(y)$ is the streamfunction's meridional structure function. Subsequently (42) becomes:

$$
i(\omega-U k)\left(\frac{d^{2} \Psi}{d y^{2}}-k^{2} \Psi\right)-i \beta k \Psi=0
$$

Although $\psi$ is important in helping understand the flow associated with forced Rossby waves, $\phi$ is required to calculate energy fluxes. An equation for $\phi$ is derived by taking $\partial / \partial x$ of (39) and $\partial / \partial y(40)$, and summing the two equations:

$$
\frac{\partial^{2} \phi}{\partial x^{2}}+\frac{\partial^{2} \phi}{\partial y^{2}}=\left(f_{o}+\beta y\right)\left(\frac{\partial^{2} \psi}{\partial x^{2}}-\frac{\partial^{2} \psi}{\partial y^{2}}\right)+\beta \frac{\partial \psi}{\partial y}-2 S \frac{\partial^{2} \psi}{\partial x^{2}}
$$


Seeking solutions for $\Phi$ represented as $\phi(x, y, t)=\operatorname{Re}\left\{\Phi(y) e^{i(\omega t-k x)}\right\}$ transforms (44) to:

$$
\frac{d^{2} \Phi}{d y^{2}}-k^{2} \Phi=\left[\left(f_{o}+\beta y\right)\left(\frac{d^{2} \Psi}{d y^{2}}-k^{2}\right)+\beta \frac{d \Psi}{d y}\right]-2 S k^{2} \Psi
$$

Equations (43) and (45) are second-order differential equations that are solved using the same Lindzen and Kuo (1969) algorithm, described in Chapter 2, with specified forcing and subject to boundary conditions, $\psi=0$, far outside the waveguide. Lastly, momentum and energy fluxes are calculated.

\section{Nondivergent Rossby-Wave Dispersion Relation}

Assuming that the meridional structure can be represented using a locally constant zonal wavenumber in the Wentzel-Kramers-Brillouin (e.g., Gill 1982) sense, (43) can be simplified into the dispersion relation for nondivergent Rossby waves, where $l$ is the meridional wavenumber:

$$
\Omega=\omega-U k=-\frac{\beta k}{k^{2}+l^{2}}
$$

For meridionally propagating Rossby waves, if $l$ becomes large, then $\Omega \rightarrow 0$ (47).

Conversely, if $l$ approaches zero, the waves are Doppler-shifted to the frequency of a 1dimensional wave, $\Omega \rightarrow-\beta / k$, effectively the cutoff frequency (48). 


$$
\begin{gathered}
\lim _{l \rightarrow \infty} \Omega=-\frac{\beta k}{k^{2}+l^{2}} \rightarrow 0 \\
\lim _{l \rightarrow 0} \Omega=-\frac{\beta k}{k^{2}+l^{2}} \rightarrow-\frac{\beta}{k} \rightarrow \Omega_{1 D}
\end{gathered}
$$

In addition, zonal and meridional phase and group velocities are obtained by dividing and differentiating (46) with respect to $k$ and $l$ :

$$
\begin{gathered}
C_{g x}=\frac{\partial \omega}{\partial k}=U-\frac{\beta\left(l^{2}-k^{2}\right)}{\left(k^{2}+l^{2}\right)^{2}} \\
C_{y}=\frac{\omega}{l}=\frac{k}{l}\left(U-\frac{\beta}{k^{2}+l^{2}}\right)=C_{x}\left(\frac{k}{l}\right)
\end{gathered}
$$

If $l=k$, then the zonal group velocity (49) is exactly equal to the mean flow, whereas the meridional phase velocity (50) is the product of the zonal phase velocity $\left(C_{x}=\omega / k\right)$ and the horizontal wavenumber ratio.

Results

Forced Waves in the Rossby Waveguide

The sinusoidal vorticity forcing imposed near the middle of the domain produces a wavetrain of perturbations (Fig 60a) that resemble observed frontal cyclones in middle latitudes (Fig 60c,d). The comma-shaped gyres have small protrusions at $y_{Q}$ and are advected eastward by the mean flow with curved tails trailing to the south. Between the 
gyres, a counterflow alternates between poleward and equatorward flow that supports exchanges of geopotential and momentum. The gyres' meridional extents highlight the boundaries of the Rossby waveguide at $\sim 3800 \mathrm{~km}$ and $900 \mathrm{~km}$ (Fig 60b).

Individual wave packets propagate away from $y_{Q}$ in the meridional waveguide. The latitude where $\Omega \rightarrow \Omega_{1 \mathrm{D}} \approx-\beta / k$ is the turning point where initially polewardpropagating waves are Doppler-shifted to the Rossby wave cutoff frequency - the highest (most negative) frequency a freely propagating Rossby wave can have. Wave energy reflects from the turning point $\left(\sim 52^{0} \mathrm{~N}\right)$ and is redirected equatorward where the waves are ultimately Doppler-shifted to zero frequency at a critical latitude $\left(\sim 29^{0} \mathrm{~N}\right)$. Energy is then filamented and absorbed at the critical latitude to produce the curved, cold-front-like tails. Rossby-wave group velocity approaches zero at the critical latitude so that dissipation and conservation of wave action are the absorption mechanisms in the linear context, rather than wave breaking (e.g., McIntyre and Palmer 1983, Lee et al. 2007, and Homeyer and Bowman 2012). Vortex Rossby waves are similarly absorbed at an outer critical radius to produce trailing rainband-like spirals. The key result is that the tails of the comma-shaped gyres form as the waves approach the critical latitude, which suggests that cold fronts trailing from frontal cyclones are vorticity filaments of absorbed Rossby waves. Another important finding is that the waves transport momentum and geopotential within the domain. 

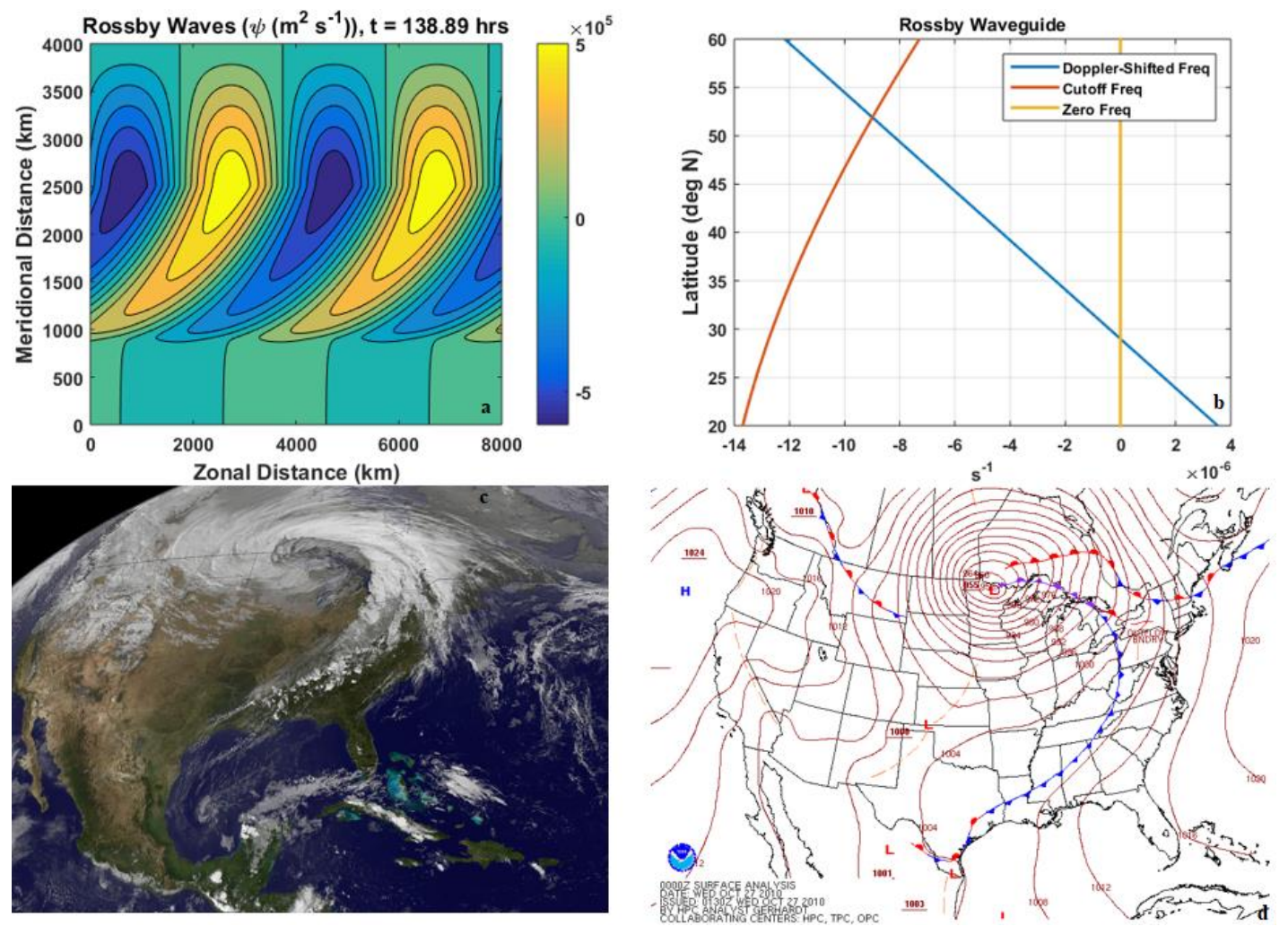

Fig 60. Streamfunction (a) depicting a wavetrain of comma-cloud-shaped gyres within an extensive meridional waveguide (b) that resembles observed frontal cyclones on satellite (c) and surface weather maps (d, source: Weather Prediction Center).

\section{Eddy Fluxes}

Analogous with VRWs from the earlier chapters, the synoptic-scale Rossby waves produce oppositely directed momentum, $\langle u v\rangle$ and geopotential fluxes, $\langle v \phi\rangle$ within the waveguide. There is a negative (equatorward) geopotential flux (Fig 61a), with the minimum centered south of $y_{Q}$. On the flanks of the waveguide, $\langle v \phi\rangle$ drops abruptly to zero from wave-energy absorption at the critical latitude and reflection from the turning point. The latter is a decaying solution, and therefore not the same for energy initially propagating southward. There is explicit wave dissipation built into the numerics for energy flux. South of $y_{Q}$, the waves transport $\phi$ equatorward, but north of 
$y_{Q}$, two counter-propagating wavetrains exist. The first transports $\phi$ poleward, until the energy reflects from the turning point, to become the second, equatorward-propagating wavetrain. Between $y_{Q}$ and the turning point, the two wavetrains cancel so that the net wave transports are zero. Equatorward of the forcing, the reflected wavetrain augments the initially equatorward-propagating wavetrain to essentially double both equatorward $\langle v \phi\rangle$ and the poleward westerly $\langle u v\rangle$.

Between $y_{Q}$ and the critical latitude, there is a poleward $\langle u v\rangle$, with a maximum centered at $y_{Q}$, and an abrupt drop to nearly zero just north of $y_{Q}$. Consequently $y_{Q}$ is a locus of $\langle u v\rangle$ convergence that acts to accelerate the westerly mean flow, whereas the critical latitude is the locus of $\langle u v\rangle$ divergence (Fig 61b) which acts to force easterly flow there. A strong convergence of westerly momentum coincides with $y_{Q}$. Absorption at the critical latitude also produces a convergence of easterly momentum. Both interactions are examples of "negative viscosity" (e.g., Starr 1968). The foregoing is consistent with conventional understanding of synoptic-scale Rossby-wave dynamics. The BND model offers particularly easy-to-comprehend explanations of Rossby-wave dynamics in sheared mean flows, the waves' role in the large-scale atmospheric circulation, and the comma shapes of observed frontal cyclones. Although the waves' structure, propagation and transport mechanics have been discussed, the waves' response to different inputs need clarification. 

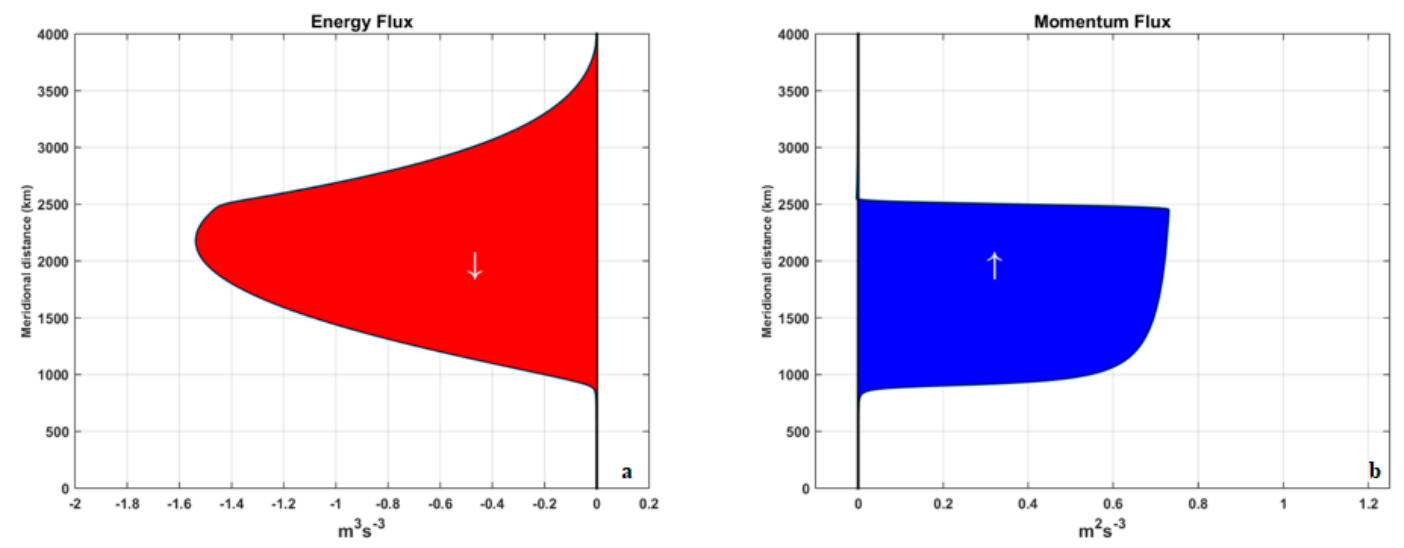

Fig 61. Horizontal fluxes of geopotential and momentum, showing that the forced Rossby waves transport westerly momentum poleward (a) and energy equatorward (b).

Waveguide Sensitivity

A key research question is, "how is the waveguide influenced by changes to specific model parameters?”. A simple series of sensitivity tests involve changes to $y_{\mathrm{o}}, S$, and $y_{Q}$. By default, the mean flow shifts from easterly to westerly at $y_{\mathrm{o}}=500 \mathrm{~km}$. If $y_{\mathrm{o}}=$ $0 \mathrm{~km}$, then $U$ becomes purely westerly over the entire domain and increases linearly with latitude $\left(0-10 \mathrm{~ms}^{-1}\right.$, Fig 62a). The forced gyres are highly asymmetric with tails extending to nearly the bottom of the domain at $\sim 400 \mathrm{~km}$, (Fig 62b) suggesting an equatorward shift of the critical latitude. To produce the most realistic meridional mean flow where there are easterly winds in the subtropics $\left(20-30^{\circ} \mathrm{N}\right)$ and westerly winds in mid-latitudes $\left(30^{0} \mathrm{~N}-60^{0} \mathrm{~N}\right), y_{\mathrm{o}}$ is set to $1000 \mathrm{~km}$ (Fig 62c). The forced comma-shaped gyres' trailing tails curve southwestward at $\sim 1400 \mathrm{~km}$, implying a poleward shift of the critical latitude (Fig 62d). A recurring result from $y_{0}$-sensitivity studies for propagating wavetrains is that the gyres' tails never extend farther southward than a point where $U<$ $1 \mathrm{~ms}^{-1}$. Lastly, if a pure easterly flow is considered, where $y_{\mathrm{o}}=4000 \mathrm{~km}, U$ decreases from -10 to $0 \mathrm{~ms}^{-1}$ with latitude (Fig 62e). The forced perturbations have a compressed 
elliptical shape (Fig 62f), giving the appearance of a standing wave structure. The waves have a positive $\Omega$, which results in the non-propagating Rossby passband lying outside the zero-frequency boundary.
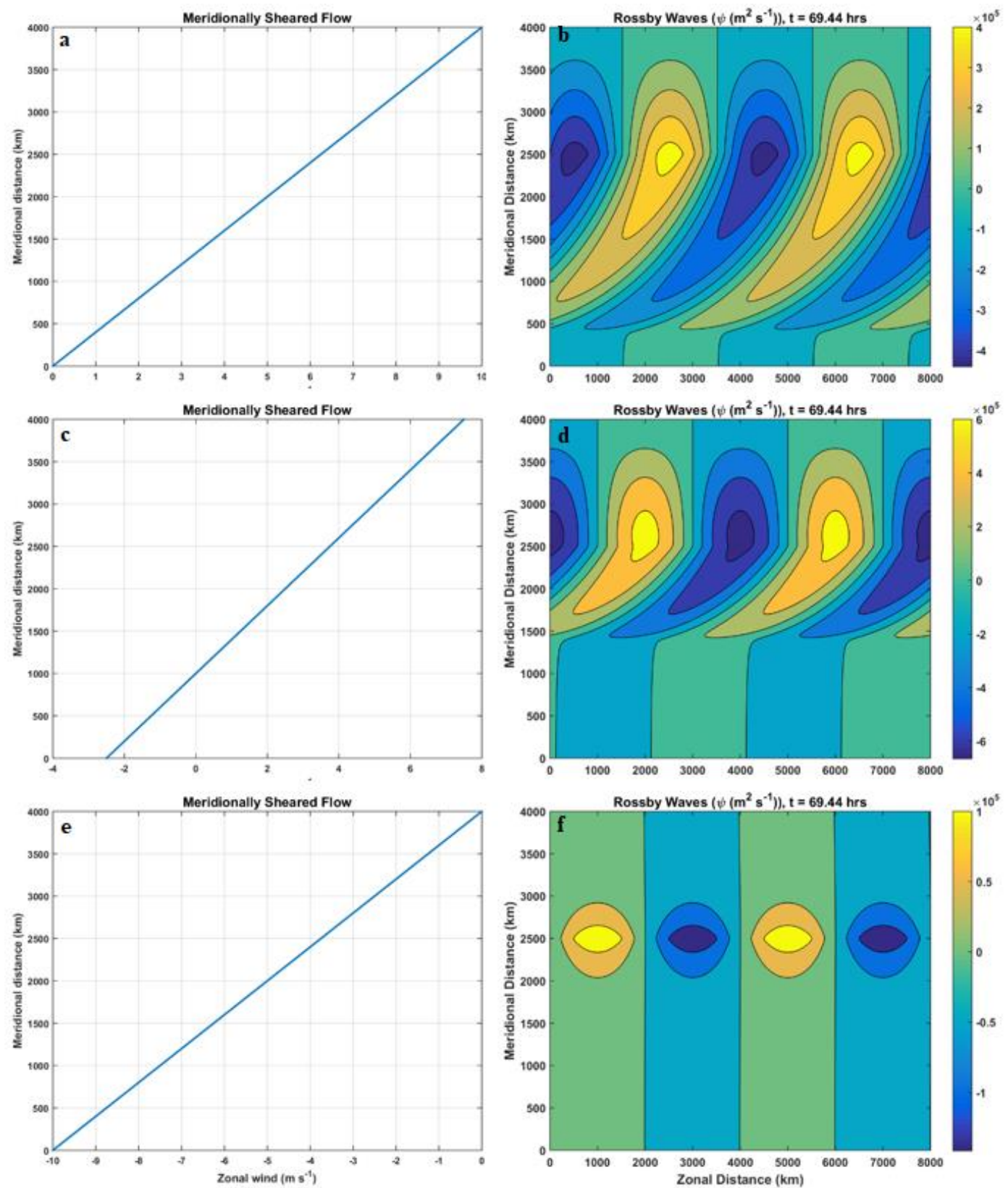

Fig 62. Mean-flow profile and forced wavetrain in pure westerly flow (top row), most realistic flow (mid row), and in pure easterly flow (bottom row). 
Changes to shear also affect the meridional wind profile. Halving $S$ to $1.25 \times 10^{-3}$ $\mathrm{s}^{-1}$ for example, substantially increases the range of $U$ from -5 to $45 \mathrm{~ms}^{-1}$ (Fig 63a). Streamfunction depicts the wavetrain as symmetric gyres with tight contours and a maximum $\psi$ amplitude centered $\sim 750 \mathrm{~km}$ north of $y_{Q}$. However, streamlines for the inflection points and cold-front-like tails between gyres are widely spaced, indicative of weak flow between $y_{Q}$ and the critical latitude (Fig 63c). If instead $S$ is doubled to $5 \times 10^{-3} \mathrm{~s}^{-1}, U$ ranges from -2.5 to $17.5 \mathrm{~ms}^{-1}$ (Fig 63b). The forced gyres appear more pronounced with the tail extending down to slightly north of $y_{\mathrm{o}}$ (Fig 63d). Shear unsurprisingly plays a role in the gyres' symmetry, with higher values yielding more asymmetric perturbations. Regardless of the shear amplitude however, the gyres' tails again, never extend further equatorward, than the critical value, $U<1 \mathrm{~ms}^{-1}$. 

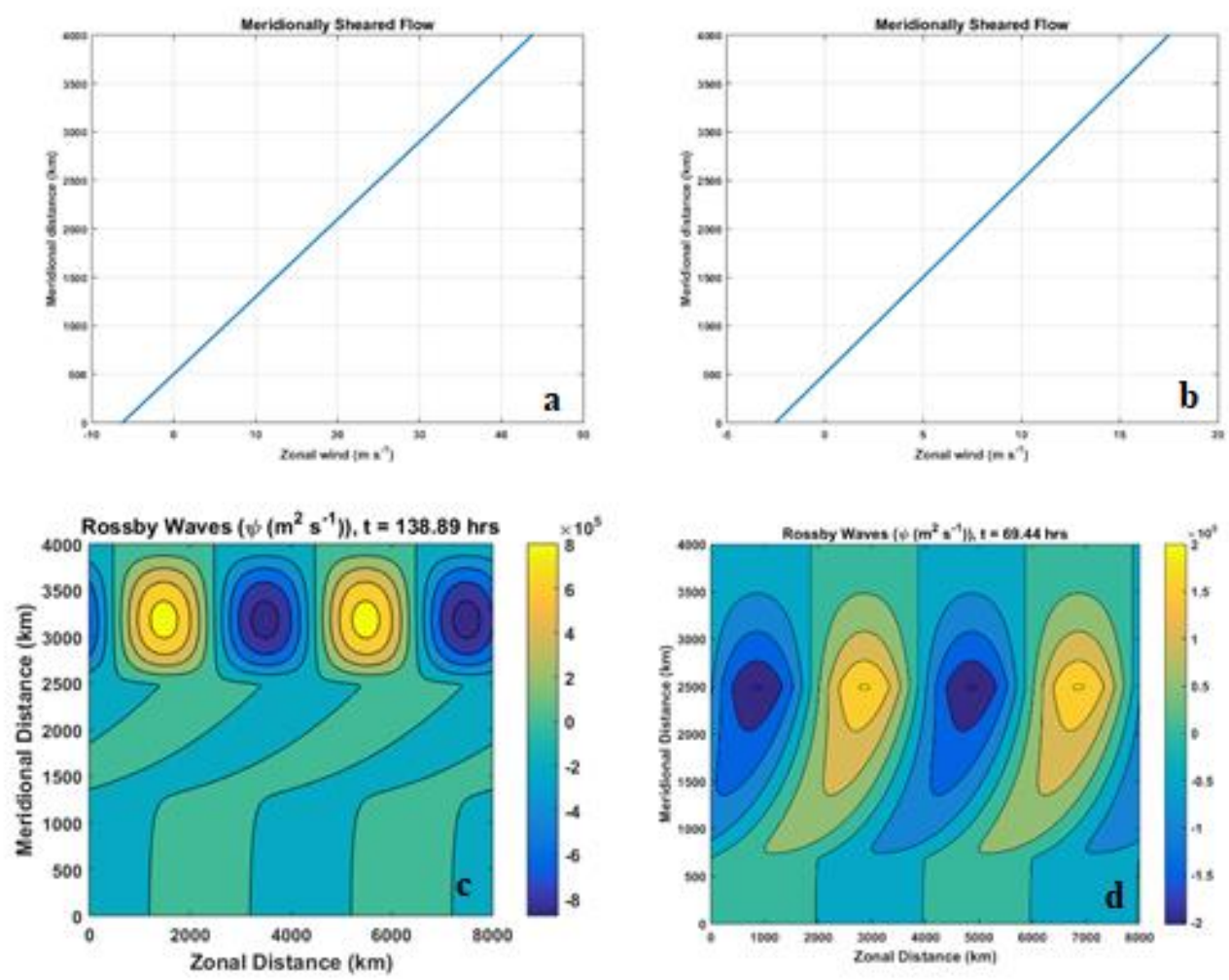

Fig 63. Mean-flow profile (a,b) and forced wavetrain (c,d) for a shear value: $1.25 \times 10^{-3} \mathrm{~s}^{-1}$ (left column); and shear value: $5 \times 10^{-3} \mathrm{~s}^{-1}$ (right column).

To conclude the sensitivity studies, $y_{Q}$ is moved to different loci. Recall that by default, $y_{Q}=2500 \mathrm{~km}\left(45^{0} \mathrm{~N}\right)$. If the waves are forced near the upper boundary of the waveguide (e.g., Fig 64a), then the $\psi$ gyres appear similar to the initial model run with the perturbations acquiring a comma-shaped structure, but with inflection points along the shifted $y_{Q}($ Fig $64 \mathrm{~b})$. For $y_{Q}=2000 \mathrm{~km}\left(40^{0} \mathrm{~N}\right)$, the wavetrain compresses slightly from an equatorward displacement of the turning point $(\sim 3500 \mathrm{~km}$; Fig $64 \mathrm{c}, \mathrm{d})$. Lastly, if the waves are forced near the lower boundary of the waveguide (e.g., Fig 64e), a standing wave structure is produced. Meridionally stretched, elliptical gyres appear confined between 3500 and $1750 \mathrm{~km}$ with zonally stretched elliptical gyres confined within the forcing latitude (Fig 64f). The reason for the standing waves is that the forcing is 
imposed in a region where the westerly mean flow is not strong enough to support Rossby-wave propagation.

Based on all the experiments conducted, the waveguide geometry depends upon the magnitude and direction of $U$. The critical latitude tends to shift poleward if the region where $U>1 \mathrm{~ms}^{-1}$ is located northward, implying that $1 \mathrm{~ms}^{-1}$ is a critical value where the cold-front-like tails do not extend beyond. Meanwhile the gyres' geometry are most influenced by shear and where the forcing is imposed. Light shear results in a more symmetric appearance and a standing-wave structure is produced if the forcing latitude is in easterly flow. Therefore freely propagating, barotropic, nondivergent Rossby waves must be forced at a latitude where the westerly mean flow is sufficiently strong, and meridional shear is moderate. 

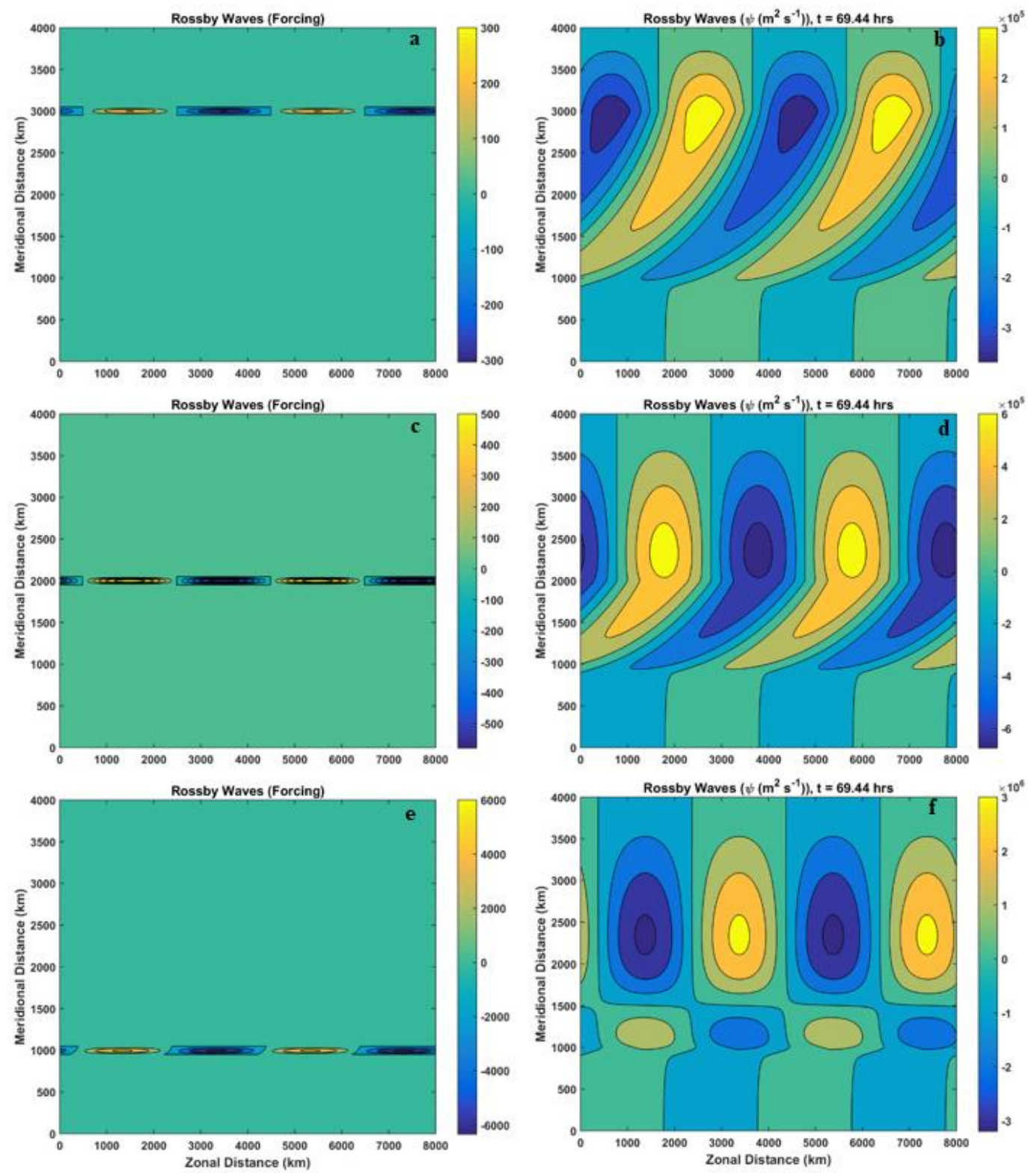

Fig 64. Forcing contour plot and forced wavetrain for a 3000-km forcing latitude (top row), 2000-km forcing latitude (mid row), and a 1000-km forcing latitude (bottom row). 


\section{Summary}

The barotropic, nondivergent framework showcases its versatility by simulating synoptic-scale, Rossby waves propagating on a meridionally sheared zonal flow. Forced waves appear as a wavetrain of alternating, comma-shaped gyres that move with the prevailing, mid-latitude westerly flow but a bit more slowly, just as vortex Rossby waves are advected downstream by tropical cyclones' mean swirling flow. The Rossby-wave gyres have long tails that curve to the southwest, much as cold fronts trailing from frontal cyclones do. Since the wavetrain exhibits alternating poleward and equatorward flow between adjacent gyres, the waves produce a poleward westerly momentum flux and an equatorward geopotential flux. The eddy fluxes are consistent with observed frontal cyclone dynamics.

Similar to vortex Rossby waves, the Rossby waveguide is defined by an environmental vorticity gradient and bounded by a turning point and critical latitude. Excited waves propagate away from the forcing latitude such that poleward wave energy is Doppler-shifted to the Rossby-wave cutoff frequency and reflects from the poleward turning point. Equatorward-propagating wave energy is Doppler-shifted to zero frequency and is absorbed at the equatorward critical latitude to form a cold-front-like tail. By comparison, outward-propagating vortex Rosby waves were shown to produce rainband-like trailing spirals in the neighborhood of the TC critical radius. The Rossbywave gyres occupy a large portion of the 4000-km meridional domain, implying that the waveguide is extensive but critical latitude can be influenced by the variation of meanflow shear. 
Sensitivity studies show that the critical latitude moves in response to changing the mean-flow zero crossing, shear, and forcing latitude. Regardless of changes to the parameters however, if the wavetrain is forced in westerly flow, the gyres' trailing tails never extend into mean flow less than $1 \mathrm{~ms}^{-1}$ which defines the critical value for wave absorption. If the waves are forced in easterly flow, the gyres take on an elliptical, standing wave structure because the Doppler-shifted frequency becomes positive, which lies outside the waveguide. Overall, sensitivity study results reinforce the concept of Rossby waves as purely synoptic, mid-latitude features embedded in westerly zonal flow. Despite successfully simulating Rossby-wave dynamics in a sheared flow, the idealized model has some unrealistic aspects. The model does not represent neither vertical wave propagation nor nonlinear wave breaking. Both are essential to understanding synoptic-scale Rossby waves' interactions with the mean flow in fullphysics models or the real atmosphere (e.g., Homeyer and Bowman 2012, McIntyre and Palmer 1983). Worthwhile future studies would involve three-dimensional analyses and jet flows. The latter is of interest because jets have more complex wind profiles where the vorticity gradient reverses sign, thus raising the possibility of barotropic instability and implying the existence of Rossby waveguides on either flank. Nevertheless, the results presented here provide a readily grasped illustration of the way Rossby waves propagate and offer an appealing explanation of the comma shape of frontal cyclones and trailing cold fronts in surface analyses. 


\section{CHAPTER VII. CONCLUSIONS}

This dissertation primarily addressed vortex Rossby waves (VRWs) in the context of tropical cyclone (TC) motion and waveguides to further synthesize the waves' dynamics and compare them with higher-wavenumber studies. Wavenumber-1 VRWs at different specified rotation frequencies, relative to the ground, were excited by rotating mass source-sink pairs imposed in the mean vortex's eyewall, in a barotropic nondivergent, vortex-following model. Chapter 3 focused on mean-vortex structural evolution, motion, inner-waveguide wave propagation, eddy fluxes, and radially trapped waves. Subsequent chapters included model sensitivity studies of forcing and vortex parameters, and repurposing the barotropic nondivergent model to simulate analogous, synoptic-scale Rossby waves. Chapter 7 revisits all the research questions asked at the beginning and concludes with suggestions for future work.

How does the mean-vortex structure evolve with time in response to rotational wavenumber-1 forcing imposed in the eyewall and what are the effects of excited VRWs on vortex motion for different specified frequencies? The model began by turning on the imposed forcing which produced a wavenumber-1 dipole in the inner core. Excited VRWs appeared as vorticity filaments propagating outward from the source, which produced a ring of tightly wrapped trailing spirals at the critical radius that resembled observed outer rainbands. The higher the specified frequency, the smaller the ring. Outside the ring was a vorticity wake caused by the combination of the mean vortex moving through a low-vorticity environment and evanescent wave energy leaking out of the inner waveguide. The vortex center's direction of motion and slipstream at any given 
time, aligned with the orientation of the wake. The streamfunction developed inner gyres surrounded by a pair of outer gyres. The latter resulted from the low-vorticity center of the mean vortex spiraling outward to produce secondary instabilities.

Vortex-center displacement naturally occurs as the low-vorticity region is pulled towards the high-vorticity eyewall. Since the forcing is imposed at the mean vortex's radius of maximum wind, and rotates with a specified frequency, trochoidal motion occurred, as observed in real TCs. The vortex center followed cyclonic orbits with the same orbital period as the forcing's rotation. However, the presence of highly filamented vorticity accumulating at the critical radius from outward-propagating VRWs created a vorticity gradient between the source and critical radius as the trailing spirals symmetrized with time. Therefore the big critical radius for low-frequency waves resulted in large trochoidal orbits.

Additionally, clarification on the waves' eddy flux budgets was obtained. As long as the low-vorticity vortex center remains in motion, angular momentum redistribution occurs, which results in eddy fluxes that drive the mean flow toward the eye as the wavenumber-1 VRW dipole evolves. Near the forcing radius, angular momentum flux converged inward and energy flux diverged outward. The former accelerated the mean flow at the radius of maximum wind, but decelerated the mean flow in the neighborhood of the critical radius. If the magnitudes of acceleration and deceleration were equal, then the result was simply a mean-flow redistribution. However, if acceleration exceeded deceleration, then wave energy was transferred from the forced dipole into the mean flow, ostensibly causing vortex intensification and storm-size expansion. For waves propagating with high specified frequencies, the radial interval of inward angular 
momentum flux convergence was confined within the small critical radius, resulting in greater mean-flow acceleration.

What is the dynamical significance of narrower or wider waveguides? Vortex Rossby waves propagate upon the mean-vortex radial vorticity gradient within the inner waveguide. The waveguide is bounded by the cutoff frequency, the highest (most negative) Doppler-shifted frequency for a given wave. The lower the waves' tangential wavenumber, the wider the waveguide, which in turn increases the range of frequencies and wave transport distances. Therefore wavenumber-1 VRWs are the least confined, have larger critical radii and are unlikely to become radially trapped. Wavenumber $>2$ waves - confined within narrow waveguides - appear less like observed, outwardpropagating tropical-cyclone rainbands.

What are the properties of radially long, radially short, evanescent, and stationary waves? Initially inward-propagating VRWs become locally long (radial wavenumber approaches zero) as the energy is Doppler-shifted to the cutoff frequency and reflected from the inner turning point. Outward-propagating waves are Doppler-shifted to zero frequency and become absorbed at outer critical radii as the waves become locally short (radial wavenumber approaches infinity). However some wave energy does leak through both waveguide boundaries, becoming evanescent tails that decay exponentially. Lastly, stationary waves have zero specified rotation frequency relative to the ground, resulting in a Doppler-shifted frequency that is equal and opposite of the mean-flow angular velocity.

What range of specified frequencies lead to radially trapped waves and what are the potential implications for vortex intensity? Although the wavenumber-1 inner 
waveguide is wide, VRWs can still become radially trapped if the specified frequency is low enough or anticyclonic. Trapping occurred when the wave energy was Dopplershifted between an inner and outer cutoff frequency, resulting in continuous wave reflection from both boundaries. However, there was no evidence to support the idea that the reflection resulted in resonant energy growth. Instead, some of the leaked energy reentered the waveguide and was eventually absorbed at the critical radius, but did not result in trailing spiral formation. Therefore it appears that unimpeded, free-wave propagation is essential to trailing spirals. Waves with an anticyclonic specified frequency resulted in clockwise trochoidal motion, because the vortex center followed the forcing's rotation, regardless of direction.

How do wavenumber-1 results compare with wavenumber $\geq 2$ ? The biggest distinctions between wavenumber-1 VRWs and higher-wavenumber variants are that the former are connected to vortex motion and propagate within the widest possible inner waveguide. Higher-wavenumber waves manifest as a wavetrain of eddies with alternating sign, advected downstream by the mean swirling flow. The streamfunction exhibits a sunflower-like pattern where the counterflow between each adjacent gyre produces a balanced inward-outward exchange of vorticity between the eye and the eyewall that does not affect vortex motion but does resemble observed eyewall mesovortices. The common theme of all barotropic nondivergent VRWs is that propagation is confined within the inner waveguide, defined by a passband between zero Doppler-shifted and the cutoff frequency. Wave reflection occurred at the inner turning point and absorption at the outer critical radius. The latter is the locus where trailing spirals form as the outward group velocity slows. Lastly, eddy fluxes carry angular 
momentum inward and energy outward from the forcing radius. Overall though, wavenumber $\leq 2$ VRWs are most like observed spiral bands because the inner waveguides are wide enough to not confine the waves within small radial intervals.

Can the present, barotropic nondivergent model verify Montgomery and Kallenbach's (1997) findings on the following: 1) excitement of VRWs near the radius of maximum wind resulted in mean-tangential-wind acceleration at the forcing locus; 2) radially broader forcing yielded a stronger response. The present model did not verify findings, but rather showed that shifting the forcing radius inward or outward from the radius of maximum wind accelerated the mean flow at the new forcing radius. Acceleration inside the radius of maximum causes eyewall contraction, whereas acceleration outside the radius of maximum wind causes eyewall expansion. Radially broadening the forcing, however, showed that a larger mean-flow acceleration radial interval at the forcing radius caused faster trochoidal motion. Since a broader forcing covered a larger area with the same intensity, the mass source-sink pair causes a stronger vortex motion. Similarly, an outwardly shifted forcing also covered more area, increasing orbital speed.

How does episodic forcing affect wavenumber-1 VRW propagation and vortex motion? When the initially continuous forcing was suddenly reduced in amplitude halfway through the simulation, wave amplitudes and vortex motion decay quickly. Conversely, stronger forcing resulted in increased vortex orbital speed and radius. Orbital radius is the product of orbital speed and the forcing's specified frequency. It is clear that the forcing's rotation induces the trochoidal motion but amplitude controls the orbital speed. Since episodic forcing experiments kept the specified frequency constant 
throughout each experiment, the vortex center orbital radius needed to shrink to decrease the orbital speed.

How does vortex motion change when a beta-forcing is added? Adding a betaforcing into the original nondivergent vorticity equation produced a more complex vortex motion, consisting of large-scale northwestward drift superimposed with small-scale wobbles. The motion resembled observed, trochoidal TC tracks. Beta gyres formed at the vortex periphery as a wavenumber- 1 dipole whose counterflow advected axially symmetric, mean-flow vorticity to the northwest, causing vortex motion in the same direction. The translation speed showed a growing high-frequency/high-amplitude oscillation that matched the forcing's specified orbital period. The beta gyres are actually VRWs propagating within the outer waveguide that are Doppler-shifted to zero frequency and absorbed at an inner critical radius. It is important to note that the rotating mass source-sink pair and beta-forcing are a linear superposition of two solutions that are purely additive with no interaction.

How does model reinitialization with added beta-forcing impact motion? The initially growing, high-frequency translation speed oscillations decayed slowly as the environment shifted from a beta-to- $f$-plane because of the reduced amplitudes of the beta gyres. Unlike the "convective" asymmetries, the beta gyres' whole-vortex spatial scale meant that the gyres filamented slowly and persisted for long times, which enabled the vortex to continue the beta drift with slow deceleration. Unaffected by the $f$-plane transitions, the inner "convectively forced" gyres continued. If instead, the rotating mass source-sink pair was suppressed while the vortex remained on a $\beta$-plane, the initially wobbly northwest track smoothed quickly. The translation speed transitioned from 
oscillatory to steady. Lastly, a reduction of the total forcing to zero resulted in a smoother translation, albeit with a steadily decreasing speed. Therefore the beta gyres exert the greatest influence on large-scale vortex motion and the rotating mass sourcesink pair is responsible for only the trochoidal oscillations.

How does the initial intensity of the mean vortex influence VRW propagation and vortex motion? Weak vortices yielded wide waveguides because climatologically weaker maximum wind correlates with larger radius of maximum wind. Despite the wider waveguide, the vorticity gradient was weak, therefore wave propagation appeared limited, as evidenced by the lack of apparent vorticity filaments and trailing spirals. A weaker imposed forcing to reflect weak convection also contributed the minimal wave activity. More intense vortices yielded narrower waveguides with strong vorticity gradients and stronger imposed forcing. Wave propagation was much more evident but the inherently high specified frequencies resulted in small critical radii, therefore confining the waves to small radial intervals and trailing spiral formation close to the eyewall. The small distance between the inner core and critical radius resulted in trochoidal motion with small orbital radii. Overall, intensity sensitivity studies demonstrated that tangential wavenumber is not the only variable that influences waveguide width; wind speed, eye size, and wind-profile shape are also important. Are mean-vorticity monopoles consistent on a spherical manifold? Bounded vortices whose circulations approach zero or become identically zero at some finite radius are consistent with Stokes' Theorem, which states that the circulation around a closed contour is equal to the total vorticity within the area enclosed. An annulus of anticyclonic vorticity must therefore surround the inner core of cyclonic vorticity, 
implying the existence of an outer waveguide because of a reversed radial vorticity gradient at the vortex periphery. Unbounded vortices however are inconsistent with Stokes' Theorem on a spherical Earth because they must have nonzero circulation outside the inner core, thus bringing about a logical contradiction. The contradiction can be avoided by having diffuse anticyclonic vorticity everywhere outside the core.

On a closed, convex manifold, the component of the curl normal to the surface must integrate to zero. Integrating from the center of a cyclonic vorticity patch outward to the patch's boundary and then to the antipode on a spherical manifold with zero vorticity outside of the patch, results in a singularity where the wind approaches infinity as the length of a contour (enclosing zero vorticity) in the neighborhood of the antipode shrinks to zero length.

How do differently shaped wind profiles of bounded and unbounded vortices compare with the default, mean vortex response from Chapter 3? Bounded and unbounded vortices generally showed a structural evolution similar to the default mean vortex from Chapter 3. However, the two finitely bounded vortices whose circulation became identically zero at $250 \mathrm{~km}$ showed a northeast-drifting trochoidal motion that may be attributed to the local vorticity gradient sign reversal. The cores of vortices therefore with small circulations may contain a weak barotropic instability that could sustain the vortex center's initial displacement to the northeast from start-up transient growth of the dipole.

What are the analogies between barotropic nondivergent VRWs and synopticscale Rossby waves and how is the waveguide influenced by changes to specified model parameters? Synoptic-scale Rossby waves share many similarities with VRWs: Both 
waves propagate freely within waveguides defined by a passband between a cutoff and zero frequency, produce oppositely directed geopotential and momentum fluxes that accelerate the mean flow at the forcing locus, manifest as a wavetrain of gyres with alternating polarity that is advected downstream by the mean flow, and form trailing spirals at a "critical point" that resemble cold fronts observed in frontal cyclones and spiral bands in TCs.

Simulated mid-latitude Rossby waves were excited by a sinusoidal vorticity forcing in a meridionally sheared, zonal flow on a 4000-km domain. The forced waves appeared as a train of comma-shaped gyres of alternating polarity that moved with the predominantly westerly mean flow. The gyres' structure resembled observed frontal cyclones with filamented cold-front-like tails, trailing equatorward. Counterflow between adjacent gyres produced an equatorward geopotential flux and poleward flux of westerly angular momentum, consistent with frontal cyclone dynamics. The latter converged at the forcing latitude to accelerate the mean flow there. Waves propagated both equatorward and poleward away from the source. They were confined within a meridional waveguide between a poleward turning point and equatorward critical latitude. Poleward-propagating waves were Doppler-shifted to the cutoff frequency and reflected from the turning point. Equatorward-propagating waves were Doppler-shifted to zero frequency and absorbed at the critical latitude.

The critical latitude is the locus where the trailing vorticity filaments curve southwestward, suggesting that "weathermaker" cold fronts in the southeast US may be trailing vorticity filaments. Lastly, sensitivity studies showed that the critical latitude can shift southward, such that the comma-like-tails becomes elongated if sufficiently strong 
westerly flow extends into the subtropical region of the domain. However, standing waves manifesting as elliptical gyres lacking the comma-shape, were produced if the shear was weak or waves were forced in easterly flow.

Validation and Future Work

This dissertation contributes to the body of VRW knowledge mainly by highlighting how the waves' propagation at different specified frequencies control vortex structural evolution and motion. Moreover, thinking about VRWs in terms of waveguides offers insight into where trailing spirals form and what controls the mean flow distribution in the vortex core. The most important output from the barotropic, nondivergent model was that simulated outward-propagating VRWs lead to outer rainband formation and TC trochoidal motion; both become crucial when the storm approaches land. Although the eyewall is the strongest part of a TC, rainbands are multiple large-scale features that may occur far from the eye and continuously lash affected areas. Based on the present work, one can speculate that inner and outer observed TC rainbands are produced by VRWs propagating outward at different frequencies. Trochoidal motion corresponds to TC eye wobbling behavior where a small track deviation can sometimes make a huge difference in impacts. Applying the logic from model, a large observed eye wobble may be attributed to VRWs propagating outward to long distances, which is achievable in a wide inner waveguide. Waveguide thinking has also helped in comprehending synoptic-scale Rossby wave propagation, 
such that the location of cold-front-like trailing spirals can be determined; an important weathermaker in the Southeast US during non-summer seasons.

In spite of the model's findings elucidating VRW and Rossby-wave dynamics mostly in terms of waveguides, there are several shortcomings. First, the model is idealized in several aspects: two-dimensional, no background flow, barotropic framework, uses a steady mean-vortex intensity, and is purely theoretical formulation with no practical applications. Therefore, future work should consider three dimensions, wave propagation in differing environmental flows, and observational validation. Three dimensions enables study of vertically propagating waves. Studying different sheared flows such as jets would offer insight into wave propagation in other waveguides besides the ones identified within a tropical-cyclone-like vortex or synoptic scale mid-latitude domains. Moreover, imposing a background flow and/or performing nonlinear, $\beta$-plane simulations can capture more realistic vortex motions. Observational comparisons are ideal follow-up projects as well. One possibility is using wind profiles of real TCs to plot the inner waveguide and compare the critical radius with the location of the storms' spiral rainbands and/or outer eyewalls. Another idea is using the present model to try matching the trochoidal motion of observed TCs with well-documented eye-wobbling behavior. Lastly, waveguide thinking could also be applied to teaching dynamic meteorology courses more effectively by using numerical methods as opposed to a traditional paper and pencil approach. 


\section{REFERENCES}

Black, M.L., J.F. Gamache, F.D. Marks Jr., C.E. Samsbury, and H.E. Willoughby, 2002: Eastern Pacific Hurricanes Jimena of 1991 and Olivia of 1994: The Effect of Vertical Shear on Structure and Intensity. Mon. Wea. Rev., 130, 2291-2312

Chan, J. C. L., and R.T. Williams, 1987: Analytical and Numerical Studies of the Betaeffect in Tropical Cyclone Motion. Part I: Zero mean flow. J. Atmos Sci., 44, 1257-1265.

Chang, E.K.M., 1998. Poleward-Propagating Angular Momentum Perturbations Induced by Zonally Symmetric Heat Sources in the Tropics. J. Atmos Sci., 55, 2229-2248.

Chen, Y., and M.K. Yau, 2001: Spiral Bands in a Simulated Hurricane. Part I: Vortex Rossby Wave Verification. J. Atmos Sci., 58, 2128-2145.

Chen, Y., and M. K. Yau, 2003: Asymmetric structures in a simulated landfalling hurricane. J. Atmos. Sci., 60, 2294-2312.

Chen, Y., G. Brunet, and M.K. Yau, 2003: Spiral Bands in a Simulated Hurricane. Part II: Wave Activity Diagnostics. J. Atmos. Sci., 60, 1239-1256.

Cotto, Amaryllis, "Intermittently Forced Vortex Rossby Waves" (2012). FIU Electronic Theses and Dissertations. 553. https://digitalcommons.fiu.edu/etd/553

Cotto, A., I. Gonzalez III, and H.E. Willoughby, 2015: Synthesis of Vortex Rossby Waves. Part I: Vortex Episodically Forced Waves in the Inner Waveguide. J. Atmos Sci., 72, 3940-3956.

Davis, H.F. Introduction to Vector Analysis (College Mathematics Series). Boston, MA: Allyn and Bacon, Inc.; 1961: 198.

DelSole 2001. A Simple Model for Transient Eddy Momentum Fluxes in the Upper Troposphere. J. Atmos Sci., 58, 3019-3035.

Dunion, J.P., C.D. Thorncroft, and C.S. Velden, 2014: The Tropical Cyclone Diurnal Cycle of Mature Hurricanes. Mon. Wea. Rev., 142, 3900-3919.

Feltgen, D. Hurricanes: Common misperceptions. National Weather Service. https://www.weather.gov/news/180409-hurricanes

Gao, Cen, "Vortex Rossby Wave Propagation in Three Dimensional Tropical-CycloneLike Baroclinic Vortices" (2016). FIU Electronic Theses and Dissertations. 2533. https://digitalcommons.fiu.edu/etd/2533

Geisler, J.E. and R.E. Dickinson, 1974: Numerical Study of an Interacting Rossby Wave and Barotropic Zonal Flow Near a Critical Level. J. Atmos Sci., 31, 946-955. 
Gill, A.E. Atmosphere-Ocean Dynamics. Cambridge, England: Academic Press, Inc., International Geophysics Series, Volume 30; 1982.

Gonzalez, I., III, H.E. Willoughby, and A. Cotto, 2015: Synthesis of Vortex Rossby Waves. Part II: Vortex Motion and Waves in the Outer Waveguide. J. Atmos Sci., 72, 3958-3974.

Held, I.M., and P.J., Phillips 1990. A Barotropic Model of the Interaction between the Hadley Cell and a Rossby Wave. J. Atmos Sci., 47, 856-869.

Homeyer, C.R. and K.P. Bowman, 2012: Rossby Wave Breaking and Transport between the Tropics and Extratropics above the Subtropical Jet. J. Atmos Sci. 70, pp. 607-626.

Holland, G.J., 1983: Tropical Cyclone Motion: Environmental Interaction Plus a Beta Effect. J. Atmos Sci., 40, 328-342.

James, I.N., 1987. Suppression of Baroclinic Instability in Horizontally Sheared Flows. J. Atmos Sci., 44, 3710-3720.

Knutson, T.R., J.L. McBride, J. Chan, K. Emanuel, G. Holland, C. Landsea, I. Held, J.P. Kossin, A.K. Srivistava, and M. Sugi, 2010: Tropical Cyclones and Climate Change, Nat. Sci., 3, 157-163, doi:10.1038/ngeo779.

Kuo, H.-C., R. T. Williams, and J.-H. Chen, 1999: A possible mechanism for the eye rotation of Typhoon Herb. J. Atmos Sci., 56, 1659-1673.

Kossin, J.P., and W.H. Schubert, 2001: Mesovortices, Polygonal Flow Patterns, and Rapid Pressure Falls in Hurricane-Like Vortices. J. Atmos Sci., 58, 2196-2209.

Landsea, C. W., 2005: "Hurricanes and global warming". Nature, 438, E11-13, doi:10.1038/nature04477.

Lawrence, M. B., and B. M. Mayfield. 1977: Satellite Observations of Trochoidal Motion During Hurricane Belle 1976. Mon. Wea Rev., 105, 1458-1461.

Lee, S., S, Son, and K. Grise, 2007. A Mechanism for the Poleward Propagatoon of Zonal Mean Flow Anomalies. J. Atmos Sci., 64, 849-868.

Lindzen, R.S., and H.L. Kuo, 1969: A Reliable Method for the Numerical Integration of a Large Class of Ordinary and Partial Differential Equations. Mon Wea Rev., 97, 732-734.

Lorenz, D.J., and D.L., Hartmann 2003. Eddy-Zonal Flow Feedback in the Northern Hemisphere Winter. J. Atmos Sci., 16, 1212-1227.

Macdonald, N.J., 1968: The Evidence for the Existence of Rossby-type waves in the Hurricane Vortex. Tellus, 20, 138-150. 
Marks, D.G. The Beta and Advection Model for Hurricane Track Forecasting. National Oceanic and Atmospheric Administration Technical Memorandum; 70, 1-89; 1992.

McIntyre, M.E. and T.N. Palmer, 1983: Breaking Planetary waves in the Stratosphere. Nature 305, pp. 593-600.

Möller, J.D., and L.J. Shapiro, 2002: Balanced Contributions to the Intensification of Hurricane Opal as diagnosed from a GFDL Model Forecast. Mon. Wea Rev., 130, 18661881.

Muramatsu, T., 1986: The Structure of Polygonal Eye of a Typhoon. J. Met Soc Jap., 64, 914-921.

Montgomery, M.T., and R.J. Kallenbach, 1997: A theory for vortex Rossby-waves and its application to spiral bands and intensity changes in hurricanes. Q.J.E. Meteorol. Soc., 123, 435-465.

Montgomery, M.T., J.D., Möller, and C.T., Nicklas, 1999: Linear and nonlinear vortex motion in an asymmetric balance shallow water model. J. Atmos. Sci., 56, 749-768.

Nolan, D.S., M.T. Montgomery, and L.D. Grasso, 2001: The Wavenumber-One Instability and Trochoidal Motion of Hurricane-like Vortices. J. Atmos Sci., 58, 32433270 .

Pielke, Jr., R. A., C. W. Landsea, M. Mayfield, J. Laver and R. Pasch, "2005: Hurricanes and Global Warming". Bull. Amer. Meteor. Soc., 86, 1571-1575.

Pielke, Jr., R. A., C. W. Landsea, M. Mayfield, J. Laver, R. Pasch, 2006: Reply to Hurricanes and Global Warming Potential Linkages and Consequences. Bull. Amer. Meteor Soc., 87, 628-631.

Randal, W.J., and I.M., Held 1991. Phase Speed Spectra of Transient Eddy Fluxes and Critical Layer Absorption. J. Atmos Sci., 48, 688-697.

Rossby, C.G. and collaborators, 1939. Relation between variations in the intensity of the zonal circulation of the atmosphere and the displacements of the semi-permanent centers of action, Ibid., 2, 38-55.

Rossby, C.G., 1940. Planetary flow patterns in the atmosphere, Quart. J. R. Met. Soc., 66, Supplement, 68-87.

Reasor, P. D., M. T. Montgomery, F. D. Marks Jr., and J. F. Gamache, 2000: Lowwavenumber structure and evolution of the hurricane inner core observed by airborne dual-Doppler radar. Mon. Wea Rev., 128, 1653-1680. 
Schubert, W.H., M.T. Montgomery, R.K. Taft, T.A. Guinn, S.R. Fulton, J.P. Kossin, and J.P. Edwards, 1999: Polygonal Eyewalls, Asymmetric Eye Contraction, and Potential Vorticity Mixing in Hurricanes. J. Atmos Sci., 56, 1197-1223.

Segund, S. Weatherman Carl-Gustaf Rossby (Man's Milieu). Time Magazine, 68-79. December 17, 1956.

Starr, V.P. Physics of Negative Viscosity Phenomenon. New York, NY: McGraw-Hill, Inc.; 1968.

Wang, Y., 2002b: Vortex Rossby Waves in a Numerically Simulated Tropical Cyclone. Part II: The Roles in Tropical Cyclone Structure and Intensity Changes. J. Atmos Sci., 59, 1239-1262.

Willoughby, H.E., J.A. Clos, and M.G. Shoreibah, 1982: Concentric Eye Walls, Secondary Wind Maxima, and The Evolution of the Hurricane Vortex. J. Atmos Sci., 39, 395-411.

Willoughby, H. E., 1988: Linear Motion of a Shallow-Water, Barotropic Vortex. J. Atmos Sci., 45, 1906-1928.

Willoughby, H. E., 1992: Linear Motion of a Shallow-Water Barotropic Vortex as an Initial-Value Problem. J. Atmos Sci., 49, 2015-2031.

Willoughby, H. E., 2011: The Golden Radius in Balanced Atmospheric Flows. Mon. Wea Rev. 139(4), 1164-1168.

Wood, V.T., and L.W. White, 2010: A New Parametric Model of Vortex TangentialWind Profiles: Development, Testing, and Verification. J. Atmos Sci., 68, 990-1006. 
VITA

ISRAEL GONZALEZ III

Born, Miami, Florida

2004-2010

B.S., Geosciences

Florida International University

Miami, Florida

2011-2014

M.S., Geosciences

Florida International University

Miami, Florida

Teaching and Research Assistant

Florida International University

Miami, Florida

$2014-2019$

Doctoral Candidate

Florida International University

Miami, Florida

Teaching and Research Assistant

Florida International University

Miami, Florida

\section{PUBLICATIONS AND PRESENTATIONS}

Cotto, A., I. Gonzalez III, and H.E. Willoughby, 2015: Synthesis of Vortex Rossby Waves. Part I: Vortex Episodically Forced Waves in the Inner Waveguide. J. Atmos Sci., 72, 3940-3956.

Gonzalez, I., III, H.E. Willoughby, and A. Cotto, 2015: Synthesis of Vortex Rossby Waves. Part II: Vortex Motion and Waves in the Outer Waveguide. J. Atmos Sci., 72, 3958-3974.

33rd Conference on Hurricanes and Tropical Meteorology, 2018, Ponte Vedra, FL.

"Convectively Forced Wavenumber-1 Vortex Rossby Waves in the Inner Waveguide".

Session 7C.4

32nd Conference on Hurricanes and Tropical Meteorology, 2016, San Juan, PR.

"Constraints on Parametric Hurricane Wind Profiles". Session 18A.10

31st Conference on Hurricanes and Tropical Meteorology, 2014, San Diego, CA. "Linear 
and Nonlinear Motion of a Barotropic Vortex”. Session 4A.6

29th Conference on Hurricanes and Tropical Meteorology, 2010, Tucson, AZ. Coauthor for presentation on "Predictability of Hurricane Activity and Impacts". Session 2A.4 